\title{
Building the housane: diastereoselective synthesis and characterization of
}

\section{bicyclo[2.1.0]pentane carboxylic acids}

Volodymyr V. Semeno, Vadym O. Vasylchenko, Bohdan V. Vashchenko, Dmytro O. Lutsenko, Rustam T. Iminov, Olesia B. Volovenko,

Dr. Oleksandr O. Grygorenko*

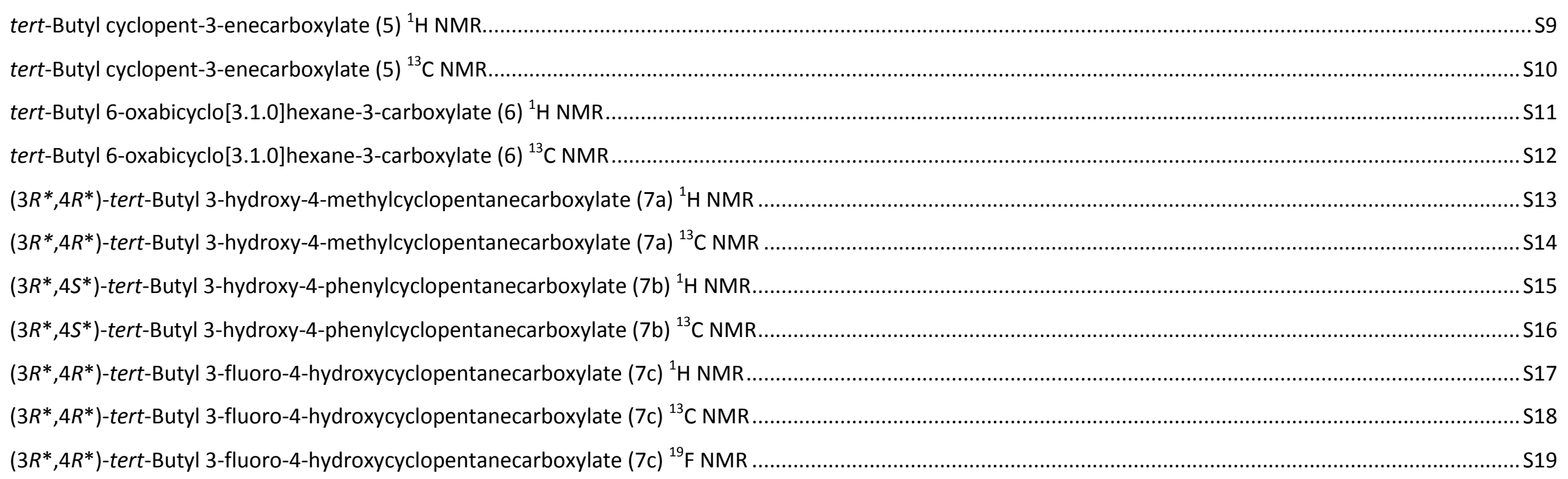


$\left(3 R^{*}, 4 R^{*}\right)$-Methyl 3-hydroxy-4-methoxycyclopentanecarboxylate $(7 \mathrm{~d}){ }^{1} \mathrm{H}$ NMR .

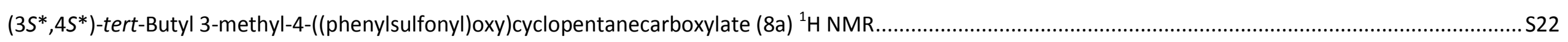

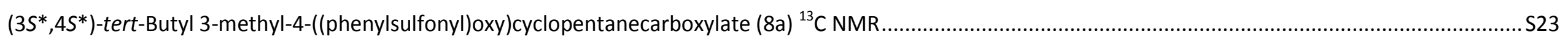

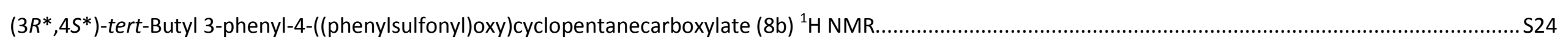

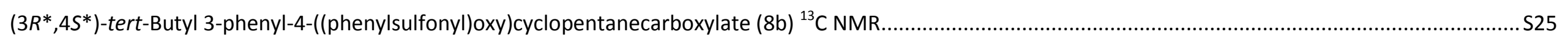

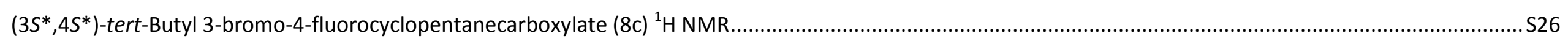

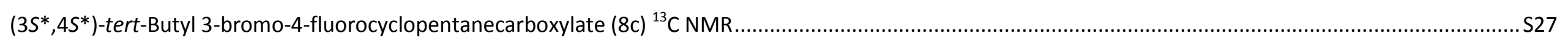

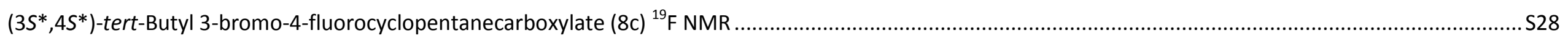

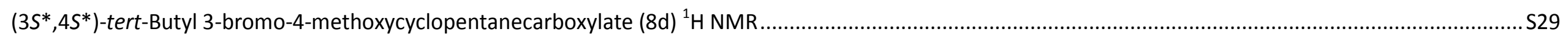

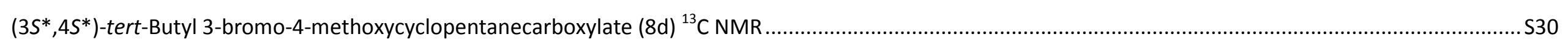

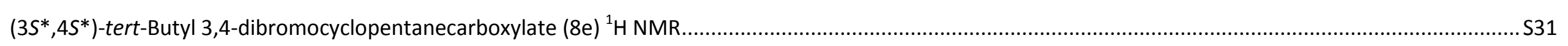

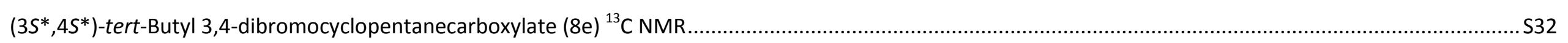

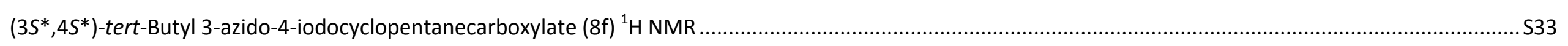

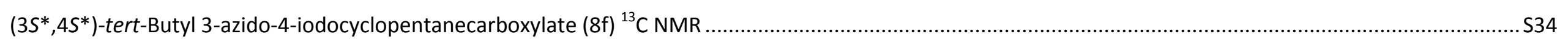

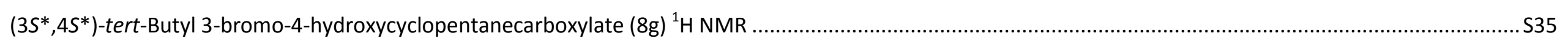

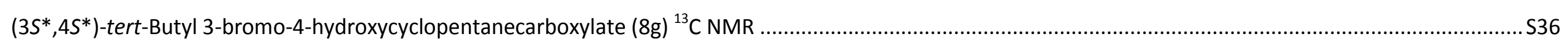

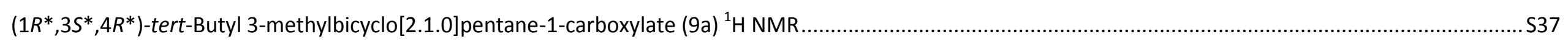

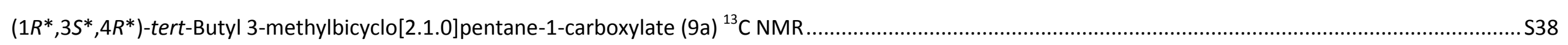

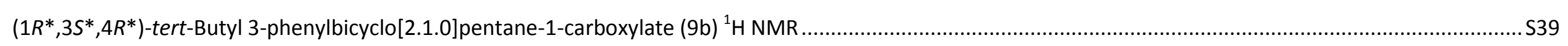

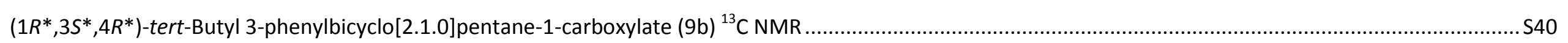

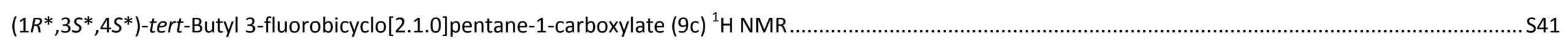


$\left(1 R^{*}, 3 S^{*}, 4 S^{*}\right)$-tert-Butyl 3-fluorobicyclo[2.1.0]pentane-1-carboxylate $(9 \mathrm{c}){ }^{13} \mathrm{C}$ NMR .

$\left(1 R^{*}, 3 S^{*}, 4 S^{*}\right)$-tert-Butyl 3-methoxybicyclo[2.1.0]pentane-1-carboxylate $(9 \mathrm{~d}){ }^{1} \mathrm{H}$ NMR

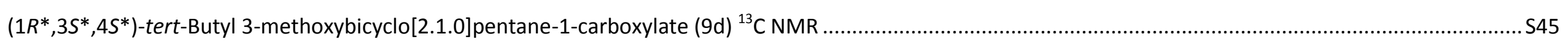

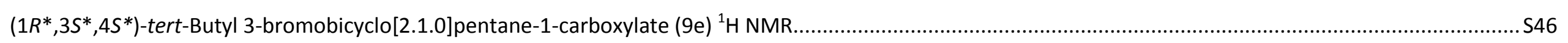

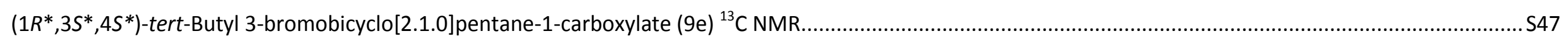

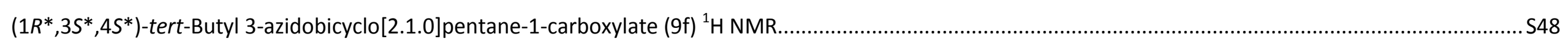

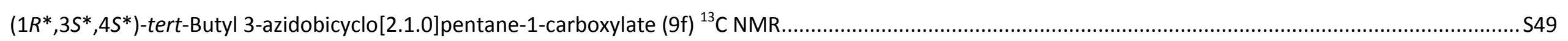

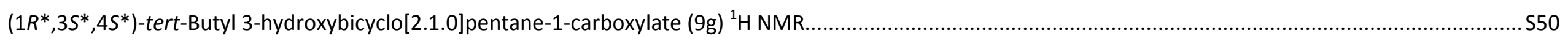

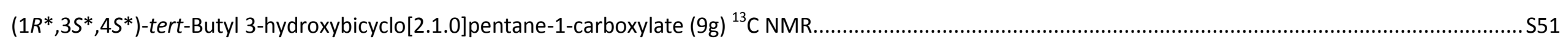

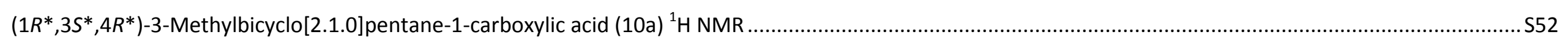

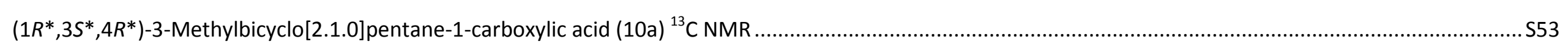

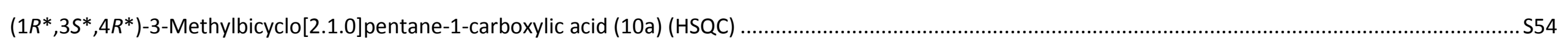

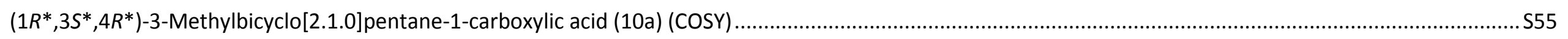

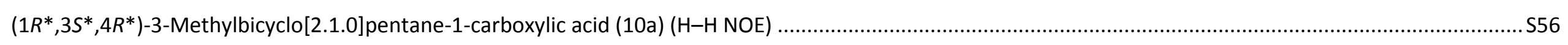

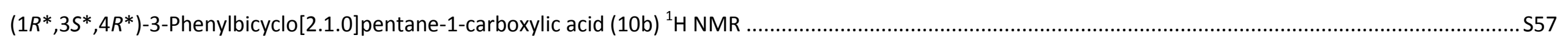

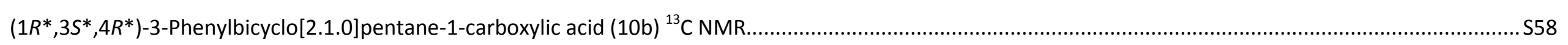

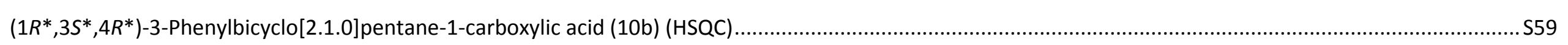

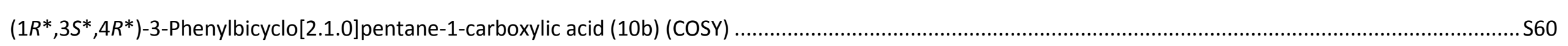

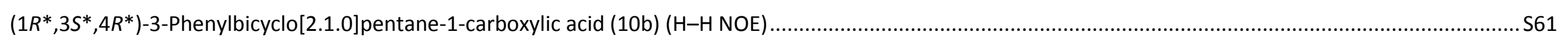

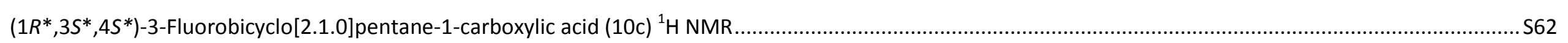

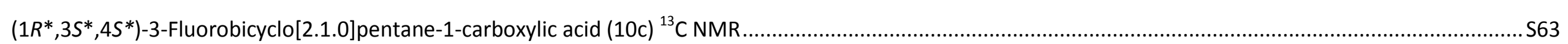


$\left(1 R^{*}, 3 S^{*}, 4 S^{*}\right)$-3-Fluorobicyclo[2.1.0]pentane-1-carboxylic acid (10c) ${ }^{19} \mathrm{~F}$ NMR

$\left(1 R^{*}, 3 S^{*}, 4 S^{*}\right)$-3-Fluorobicyclo[2.1.0]pentane-1-carboxylic acid (10c) (COSY).

$\left(1 R^{*}, 3 S^{*}, 4 S^{*}\right)$-3-Fluorobicyclo[2.1.0]pentane-1-carboxylic acid (10c) (HSQC).

$\left(1 R^{*}, 3 S^{*}, 4 S^{*}\right)$-3-Fluorobicyclo[2.1.0]pentane-1-carboxylic acid (10c) (NOESY) ..

$\left(1 R^{*}, 3 S^{*}, 4 S^{*}\right)$-3-Fluorobicyclo[2.1.0]pentane-1-carboxylic acid (10c) (HOESY).....

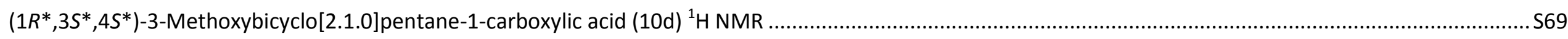

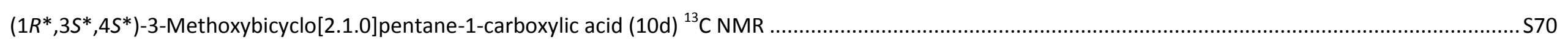

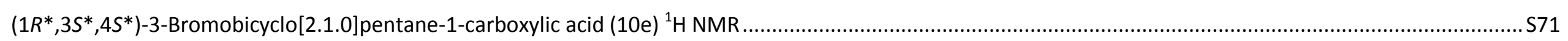

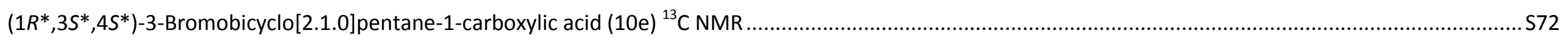

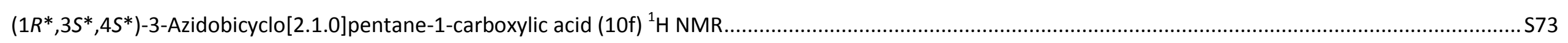

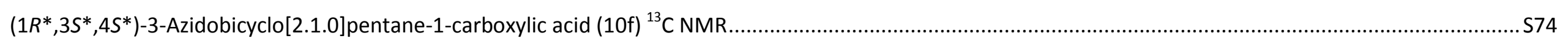

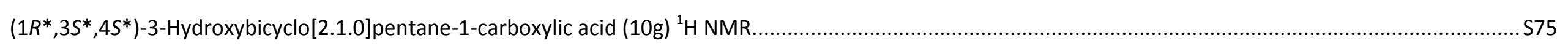

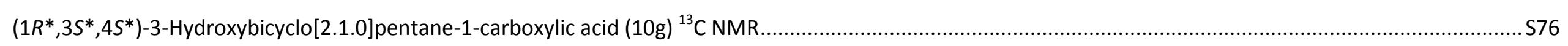

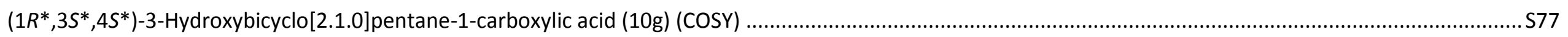

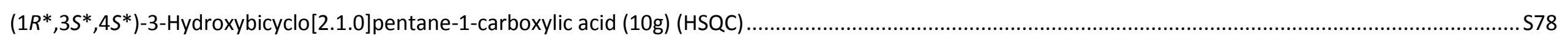

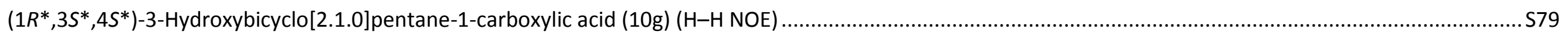

$\left(3 S^{*}, 4 S^{*}\right)$-tert-Butyl 3-bromo-4-((tert-butyldimethylsilyl)oxy)cyclopentanecarboxylate $(11 \mathrm{~g})^{1} \mathrm{H}$ NMR ............................................................................. S80

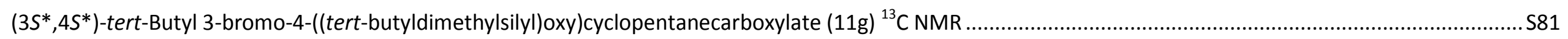

$\left(1 R^{*}, 3 S^{*}, 4 S^{*}\right)$-tert-Butyl 3-((tert-butyldimethylsilyl)oxy)bicyclo[2.1.0]pentane-1-carboxylate $(12 \mathrm{~g})^{1} \mathrm{H}$ NMR ............................................................................. S82

$\left(1 R^{*}, 3 S^{*}, 4 S^{*}\right)$-tert-Butyl 3-((tert-butyldimethylsilyl)oxy)bicyclo[2.1.0]pentane-1-carboxylate $(12 \mathrm{~g}){ }^{13} \mathrm{C} \mathrm{NMR} \mathrm{\ldots .......................................................................} 883$

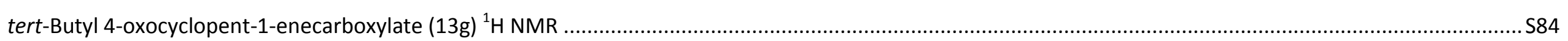

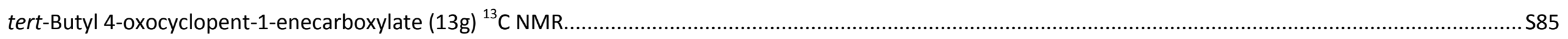


$\left(3 S^{*}, 4 R^{*}\right)$-tert-Butyl 3-bromo-4-methylcyclopentanecarboxylate (15a) ${ }^{1} \mathrm{H}$ NMR.

$\left(3 S^{*}, 4 R^{*}\right)$-tert-Butyl 3-bromo-4-methylcyclopentanecarboxylate (15a) ${ }^{13} \mathrm{C}$ NMR.

$\left(3 S^{*}, 4 S^{*}\right)$-tert-Butyl 3-bromo-4-phenylcyclopentanecarboxylate (15b) ${ }^{1} \mathrm{H}$ NMR

$\left(3 S^{*}, 4 S^{*}\right)$-tert-Butyl 3-bromo-4-phenylcyclopentanecarboxylate $(15 \mathrm{~b}){ }^{13} \mathrm{C}$ NMR S89

$\left(3 S^{*}, 4 R^{*}\right)$-tert-Butyl 3-bromo-4-fluorocyclopentanecarboxylate $(15 \mathrm{c}){ }^{1} \mathrm{H}$ NMR S90

$\left(35^{*}, 4 R^{*}\right)$-tert-Butyl 3-bromo-4-fluorocyclopentanecarboxylate (15c) ${ }^{13} \mathrm{C} \mathrm{NMR}$

$\left(35^{*}, 4 R^{*}\right)$-tert-Butyl 3-bromo-4-fluorocyclopentanecarboxylate $(15 \mathrm{c}){ }^{19} \mathrm{~F} N \mathrm{NM}$. .. 992

$\left(35^{*}, 4 R^{*}\right)$-methyl 3-bromo-4-methoxycyclopentanecarboxylate (15d) ${ }^{1} \mathrm{H}$ NMR.

$\left(3 S^{*}, 4 R^{*}\right)$-methyl 3-bromo-4-methoxycyclopentanecarboxylate $(15 \mathrm{~d}){ }^{13} \mathrm{C} \mathrm{NMR}$

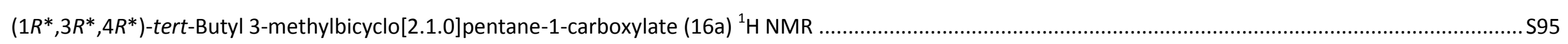

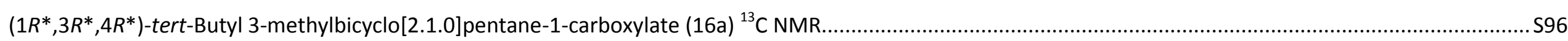

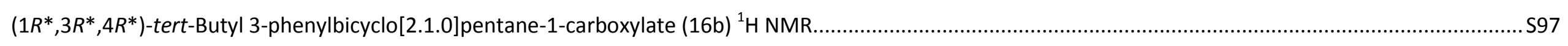

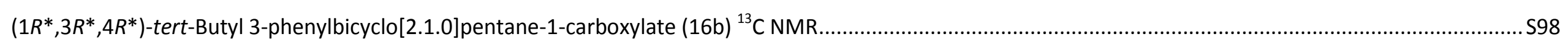

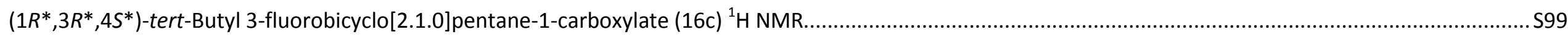

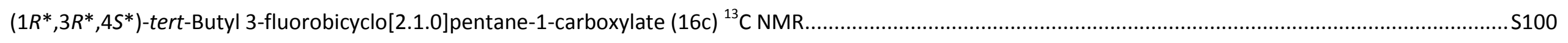

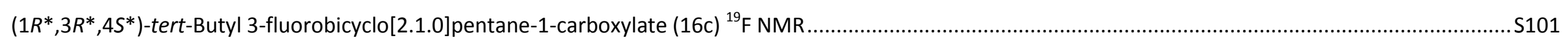

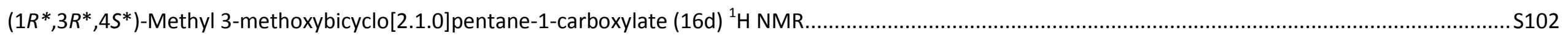

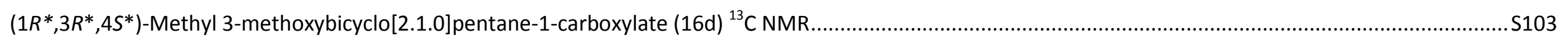

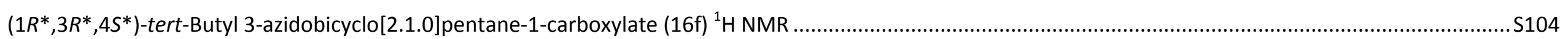

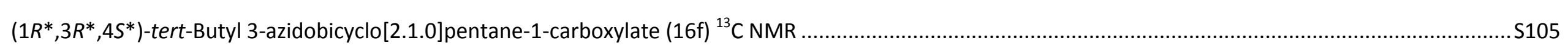

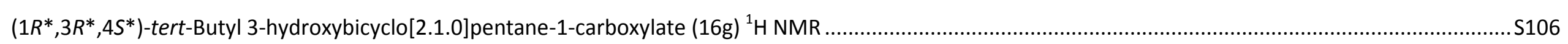

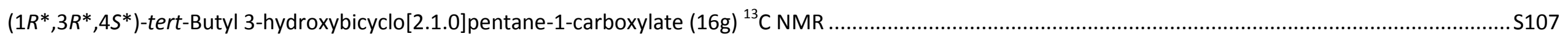


$\left(1 R^{*}, 3 R^{*}, 4 R^{*}\right)-3-M e t h y l b i c y c l o[2.1 .0]$ pentane-1-carboxylic acid (17a) ${ }^{1} \mathrm{H}$ NMR

$\left(1 R^{*}, 3 R^{*}, 4 R^{*}\right)$-3-Methylbicyclo[2.1.0]pentane-1-carboxylic acid (17a) ${ }^{13} \mathrm{C} N \mathrm{NMR}$

$\left(1 R^{*}, 3 R^{*}, 4 R^{*}\right)$-3-Methylbicyclo[2.1.0]pentane-1-carboxylic acid (17a) (COSY)...

$\left(1 R^{*}, 3 R^{*}, 4 R^{*}\right)$-3-Methylbicyclo[2.1.0]pentane-1-carboxylic acid (17a) (HSQC) .

$\left(1 R^{*}, 3 R^{*}, 4 R^{*}\right)$-3-Methylbicyclo[2.1.0]pentane-1-carboxylic acid (17a) (H-H NOE)

$\left(1 R^{*}, 3 R^{*}, 4 R^{*}\right)$-3-Phenylbicyclo[2.1.0]pentane-1-carboxylic acid (17b) ${ }^{1} \mathrm{H}$ NMR

$\left(1 R^{*}, 3 R^{*}, 4 R^{*}\right)-3-P h e n y l b i c y c l o[2.1 .0]$ pentane-1-carboxylic acid (17b) ${ }^{13} \mathrm{C} N \mathrm{NM}$

$\left(1 R^{*}, 3 R^{*}, 4 R^{*}\right)$-3-Phenylbicyclo[2.1.0]pentane-1-carboxylic acid (17b) (COSY)

$\left(1 R^{*}, 3 R^{*}, 4 R^{*}\right)$-3-Phenylbicyclo[2.1.0]pentane-1-carboxylic acid (17b) (HSQC) .....

$\left(1 R^{*}, 3 R^{*}, 4 R^{*}\right)$-3-Phenylbicyclo[2.1.0]pentane-1-carboxylic acid (17b) (H-H NOE)

$\left(1 R^{*}, 3 R^{*}, 4 S^{*}\right)-3-M e t h o x y b i c y c l o[2.1 .0]$ pentane-1-carboxylic acid $(17 \mathrm{~d}){ }^{1} \mathrm{H}$ NMR

$\left(1 R^{*}, 3 R^{*}, 4 S^{*}\right)-3-M e t h o x y b i c y c l o[2.1 .0]$ pentane-1-carboxylic acid $(17 \mathrm{~d}){ }^{13} \mathrm{C}$ NMR

$\left(1 R^{*}, 3 R^{*}, 4 S^{*}\right)-3-A z i d o b i c y c l o[2.1 .0]$ pentane-1-carboxylic acid (17f) ${ }^{1} \mathrm{H}$ NMR

$\left(1 R^{*}, 3 R^{*}, 4 S^{*}\right)-3-$ Azidobicyclo[2.1.0]pentane-1-carboxylic acid (17f) ${ }^{13} \mathrm{C}$ NMR

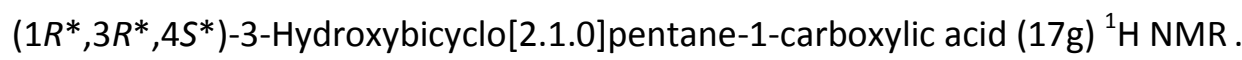

$\left(1 R^{*}, 3 R^{*}, 4 S^{*}\right)-3-H y d r o x y b i c y c l o[2.1 .0]$ pentane-1-carboxylic acid $(17 \mathrm{~g}){ }^{13} \mathrm{C}$ NMR

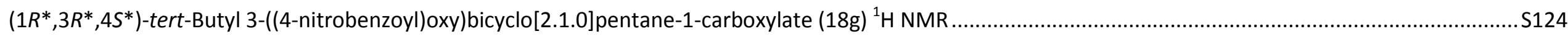

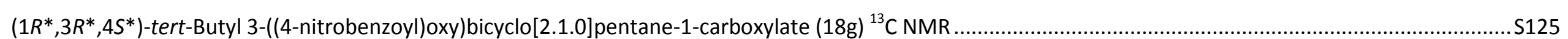

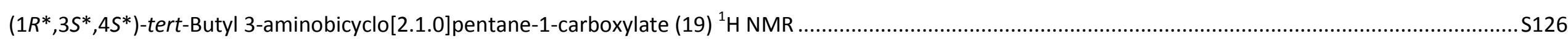

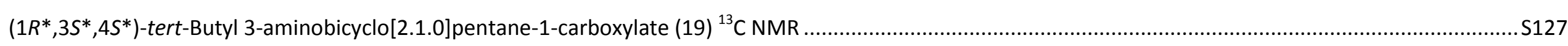

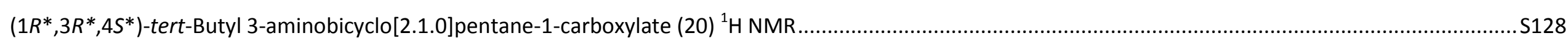

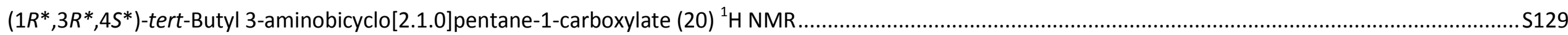


$\left(1 S^{*}, 2 S^{*}, 4 R^{*}\right)$-4-Carboxybicyclo[2.1.0]pentan-2-aminium 2,2,2-trifluoroacetate (21) ${ }^{1} \mathrm{H}$ NMR

$\left(1 S^{*}, 2 S^{*}, 4 R^{*}\right)$-4-Carboxybicyclo[2.1.0] pentan-2-aminium 2,2,2-trifluoroacetate (21) ${ }^{19} \mathrm{~F}$ NMR.

$\left(1 S^{*}, 2 S^{*}, 4 R^{*}\right)$-4-Carboxybicyclo[2.1.0] pentan-2-aminium 2,2,2-trifluoroacetate (21) (COSY).....

$\left(1 S^{*}, 2 S^{*}, 4 R^{*}\right)$-4-Carboxybicyclo[2.1.0]pentan-2-aminium 2,2,2-trifluoroacetate (21) (HSQC).

$\left(1 S^{*}, 2 S^{*}, 4 R^{*}\right)$-4-Carboxybicyclo[2.1.0]pentan-2-aminium 2,2,2-trifluoroacetate (21) (H-H NOE).

$\left(1 S^{*}, 2 R^{*}, 4 R^{*}\right)$-4-Carboxybicyclo[2.1.0]pentan-2-aminium 2,2,2-trifluoroacetate (22) ${ }^{1} \mathrm{H}$ NMR.

$\left(1 S^{*}, 2 R^{*}, 4 R^{*}\right)$-4-Carboxybicyclo[2.1.0]pentan-2-aminium 2,2,2-trifluoroacetate (22) ${ }^{13} \mathrm{C}$ NMR 
tert-Butyl 3-bromocyclopentanecarboxylate (30) ${ }^{13} \mathrm{C}$ NMR

tert-Butyl bicyclo[2.1.0]pentane-1-carboxylate (31) ${ }^{1} \mathrm{H}$ NMR

tert-Butyl bicyclo[2.1.0]pentane-1-carboxylate (31) ${ }^{1} \mathrm{H}$ NMR

cis-4-Carboxybicyclo[2.1.0]pentan-2-aminium chloride $((1 S, 2 S, 4 R)-23$ or $(1 R, 2 R, 4 S)-23){ }^{1} \mathrm{H}$ NMR.

cis-4-Carboxybicyclo[2.1.0]pentan-2-aminium chloride $((1 S, 2 S, 4 R)-23$ or $(1 R, 2 R, 4 S)-23){ }^{13} \mathrm{C} N \mathrm{NM}$...

trans-4-Carboxybicyclo[2.1.0] pentan-2-aminium chloride $((1 S, 2 R, 4 R)-24$ or $(1 R, 2 S, 4 S)-24){ }^{1} \mathrm{H} N M R$......

trans-4-Carboxybicyclo[2.1.0]pentan-2-aminium chloride $((1 S, 2 R, 4 R)-24$ or $(1 R, 2 S, 4 S)-24){ }^{13} \mathrm{C} N \mathrm{NR}$

$\left(1 R^{*}, 3 S^{*}, 4 R^{*}\right)$-3-phenylbicyclo[2.1.0]pentane-1-carboxylic acid 10b (conformer A) ORTEP diagrams.

S159

$\left(1 R^{*}, 3 S^{*}, 4 R^{*}\right)$-3-phenylbicyclo[2.1.0]pentane-1-carboxylic acid 10b (conformer B) ORTEP diagrams.

$\left(1 R^{*}, 3 R^{*}, 4 R^{*}\right)-3$-phenylbicyclo[2.1.0]pentane-1-carboxylic acid 17b ORTEP diagrams.....

$(1 S, 2 S, 4 R)$-3-aminobicyclo[2.1.0]pentane-1-carboxylic acid hydrochloride $(1 S, 2 S, 4 R)$-21 ORTEP diagrams

$(1 R, 2 R, 4 S)$-3-aminobicyclo[2.1.0]pentane-1-carboxylic acid hydrochloride $(1 R, 2 R, 4 S)$-21 ORTEP diagrams

$(1 S, 2 R, 4 R)$-3-aminobicyclo[2.1.0]pentane-1-carboxylic acid hydrochloride $(1 S, 2 R, 4 R)$-21 ORTEP diagrams

$(1 R, 2 S, 4 S)$-3-aminobicyclo[2.1.0]pentane-1-carboxylic acid hydrochloride $(1 R, 2 S, 4 S)$-21 ORTEP diagrams 

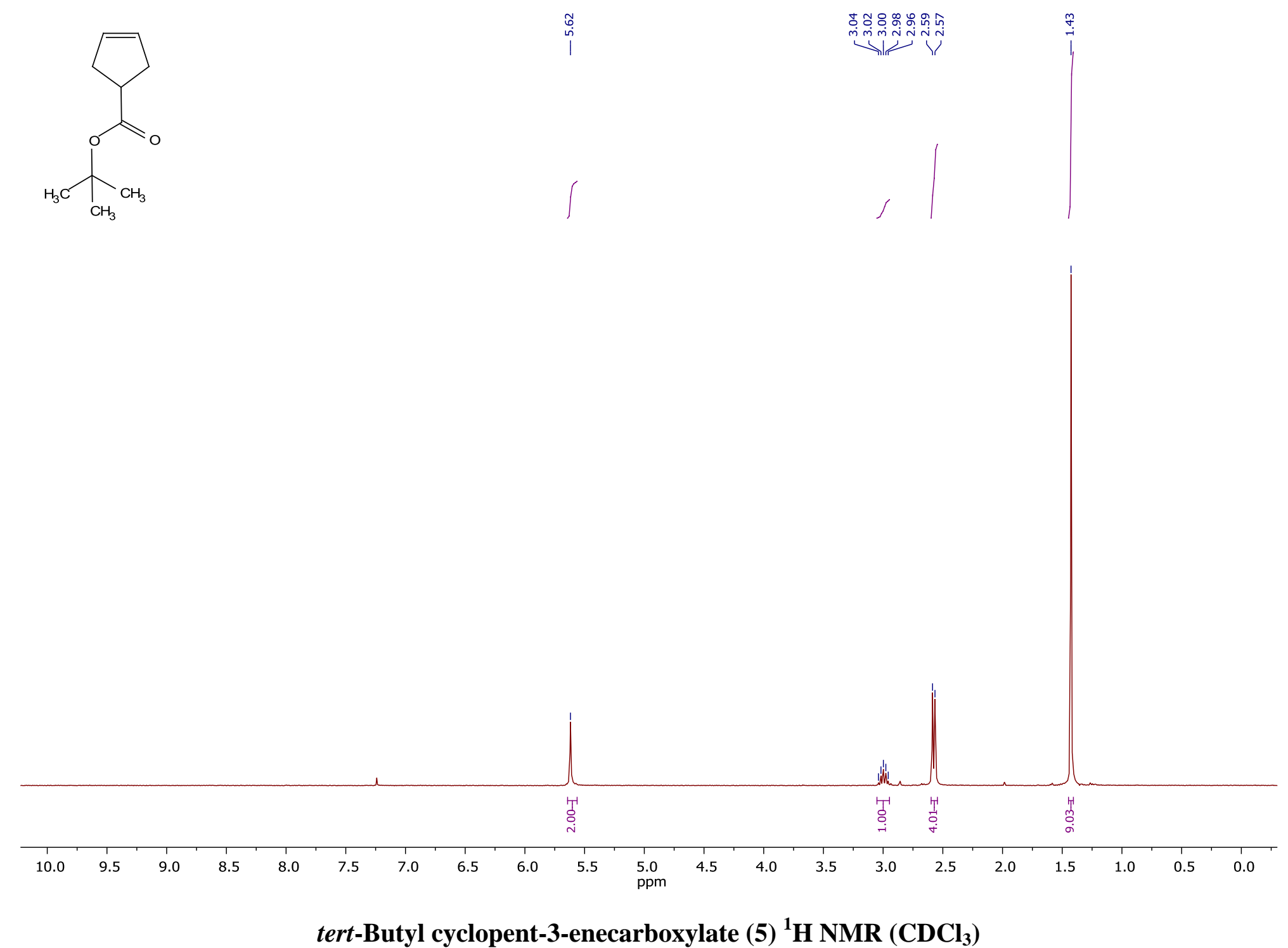

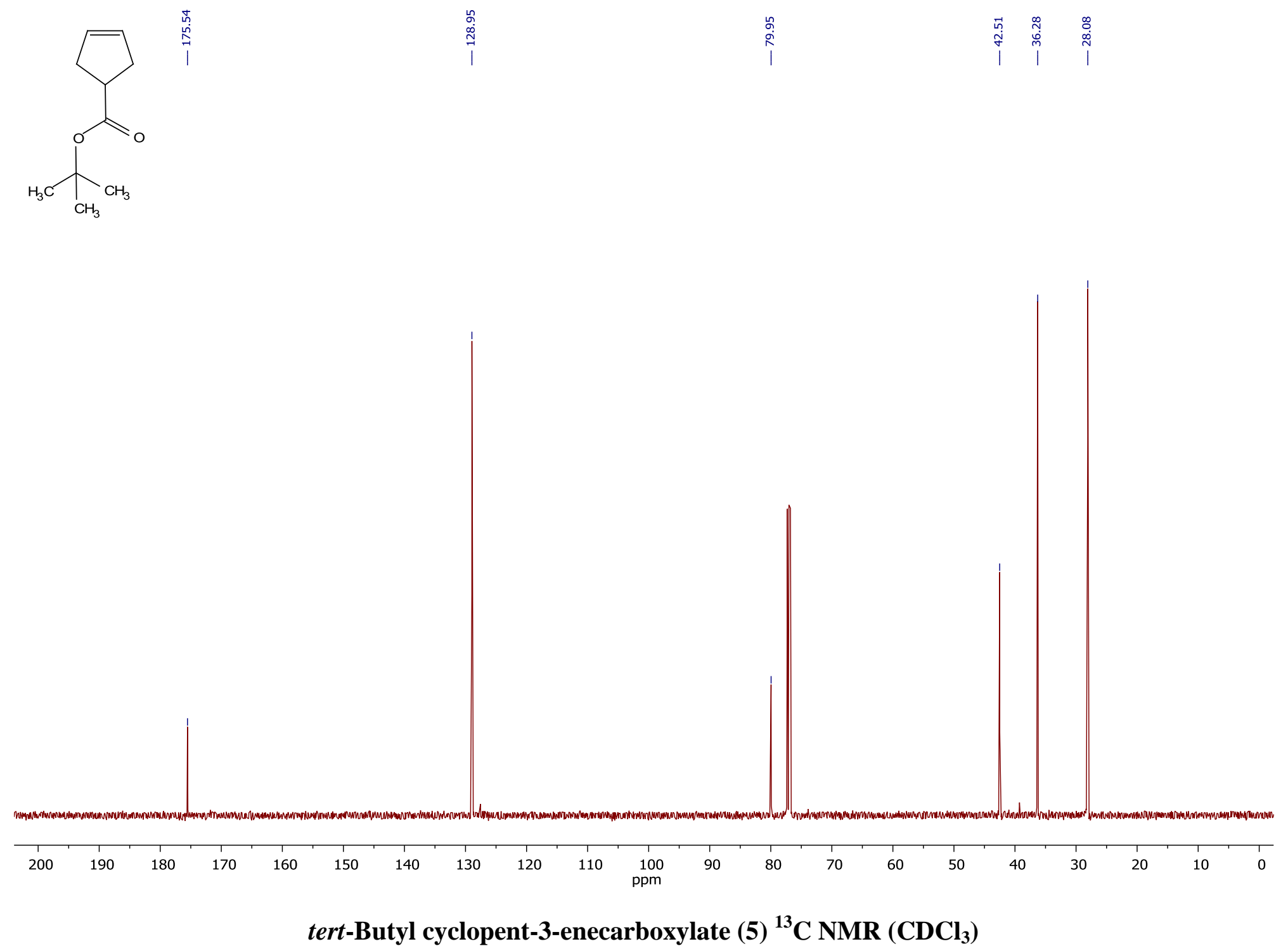

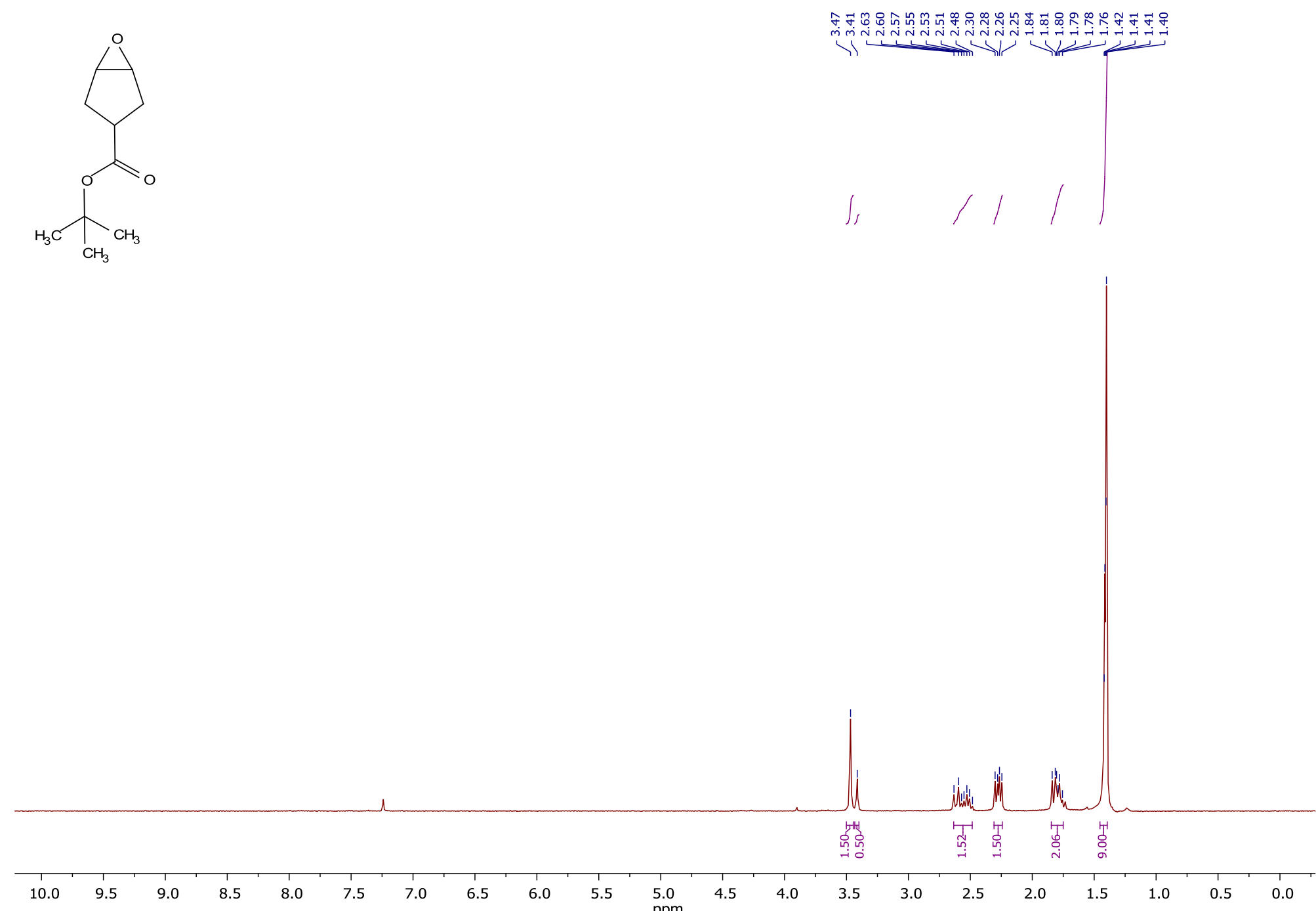

tert-Butyl 6-oxabicyclo[3.1.0]hexane-3-carboxylate (6) ${ }^{1} \mathrm{H}$ NMR $\left(\mathrm{CDCl}_{3}\right)$ 

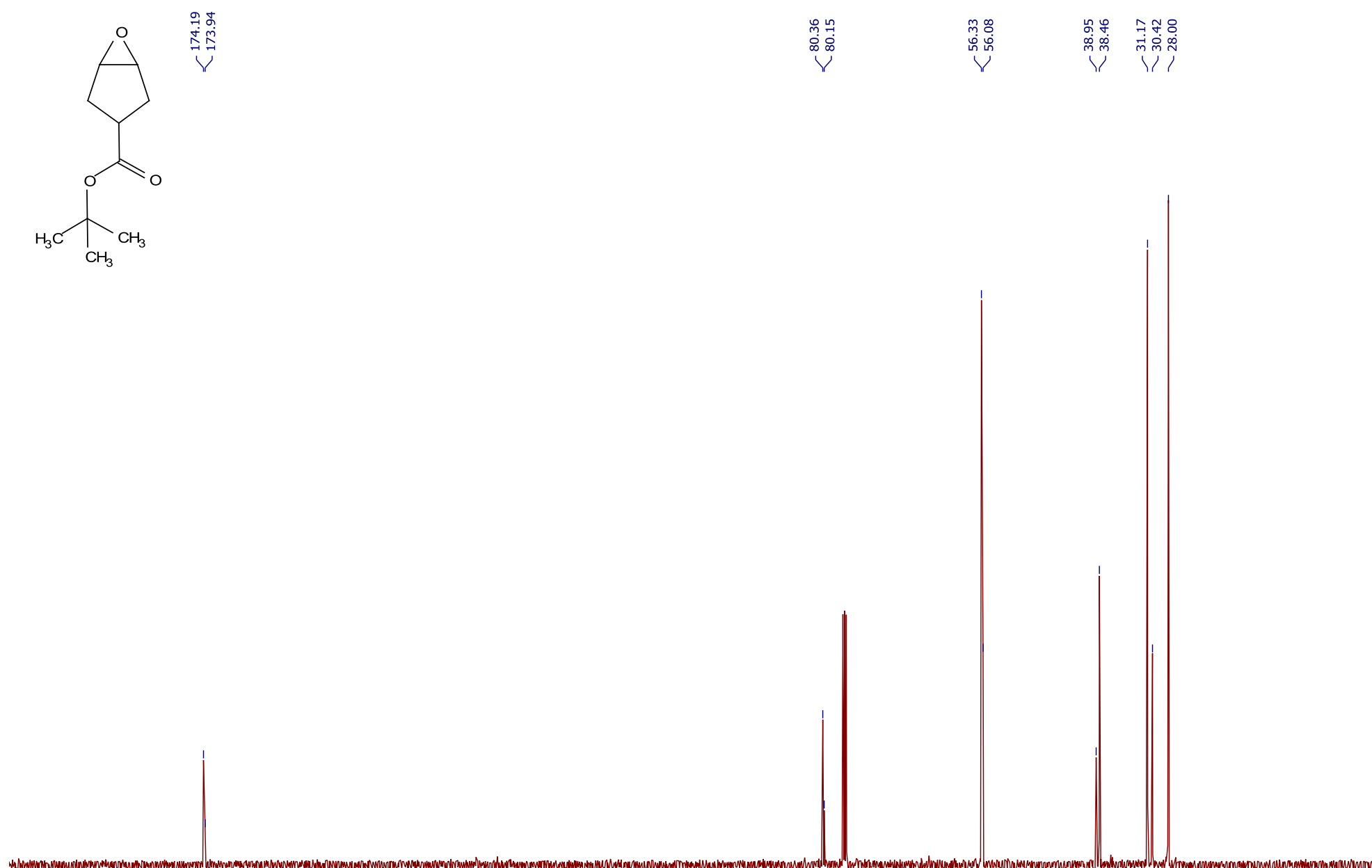

tert-Butyl 6-oxabicyclo[3.1.0]hexane-3-carboxylate (6) ${ }^{13} \mathrm{C}$ NMR $\left(\mathrm{CDCl}_{3}\right)$ 


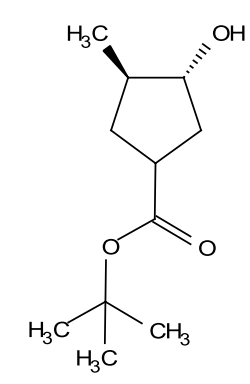

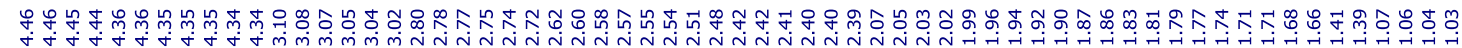

$\mathrm{H}_{3} \mathrm{C}$
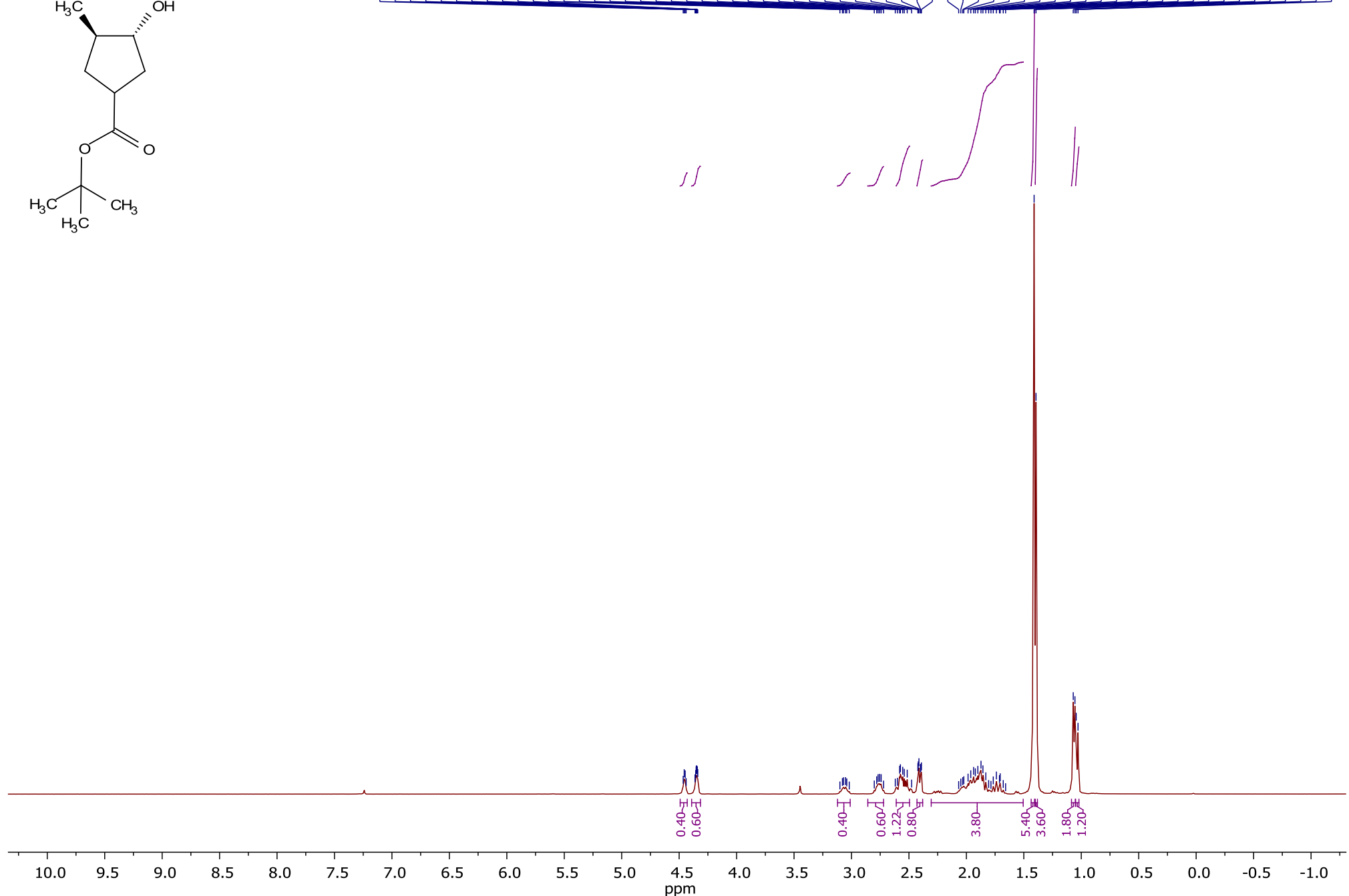

$\left(3 R^{*}, 4 R^{*}\right)$-tert-Butyl 3-hydroxy-4-methylcyclopentanecarboxylate (7a) ${ }^{1} \mathrm{H}$ NMR $\left(\mathrm{CDCl}_{3}\right)$ 

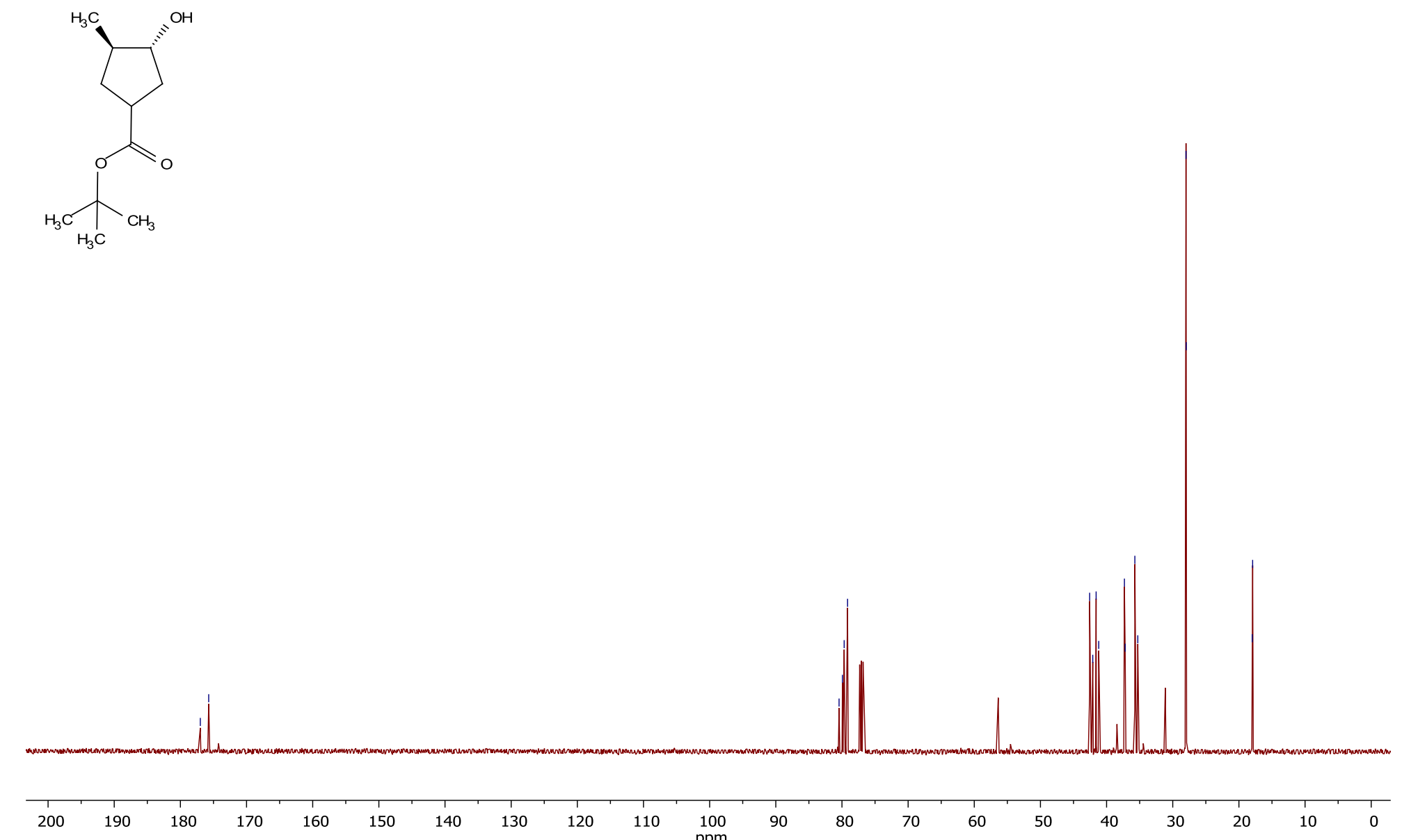

$\left(3 R^{*}, 4 R^{*}\right)$-tert-Butyl 3-hydroxy-4-methylcyclopentanecarboxylate (7a) ${ }^{13} \mathrm{C} \mathrm{NMR}\left(\mathrm{CDCl}_{3}\right)$ 

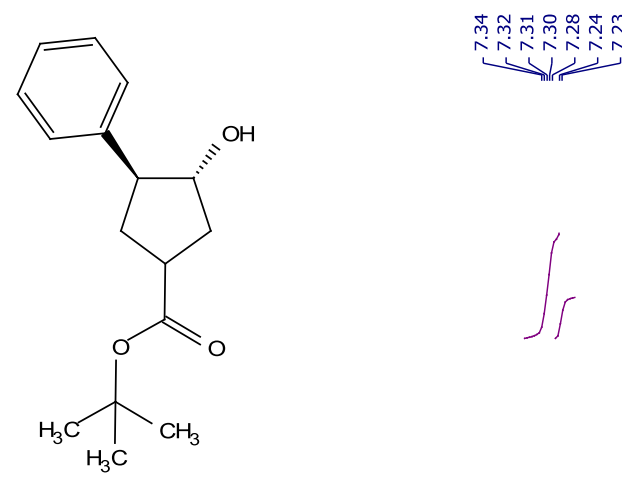

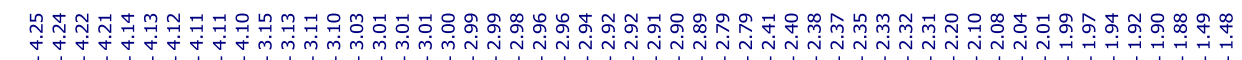

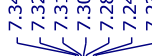

$\mathrm{H}_{3} \mathrm{C}$

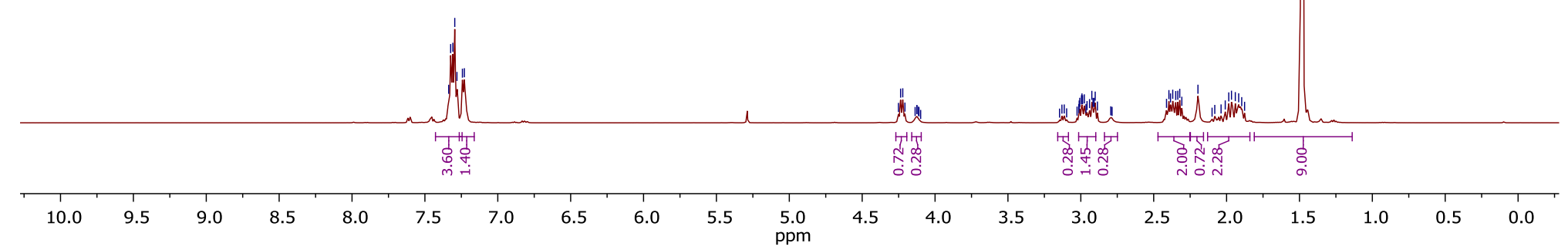

$\left(3 R^{*}, 4 S^{*}\right)$-tert-Butyl 3-hydroxy-4-phenylcyclopentanecarboxylate (7b) ${ }^{1} \mathrm{H}$ NMR $\left(\mathrm{CDCl}_{3}\right)$ 


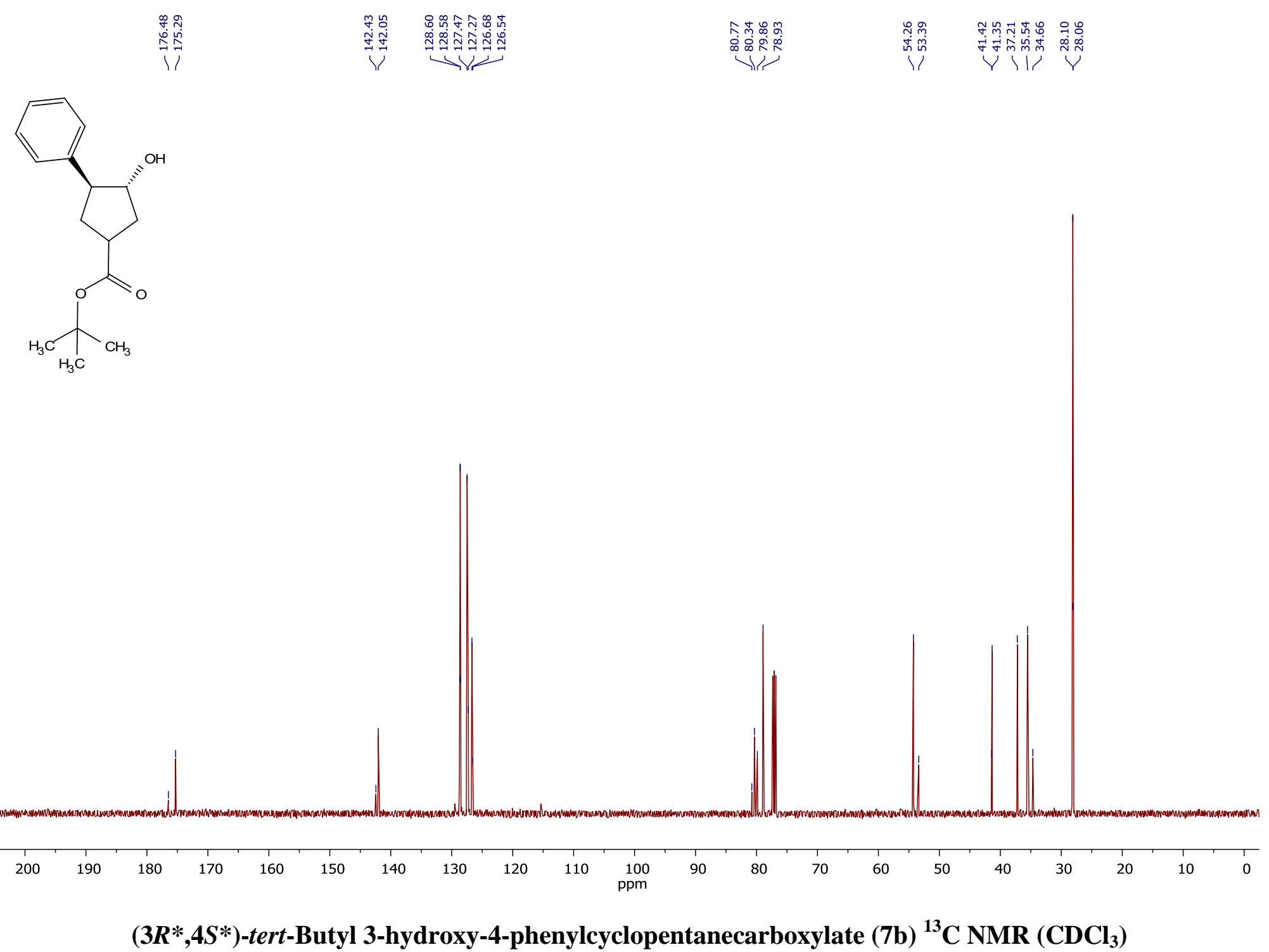




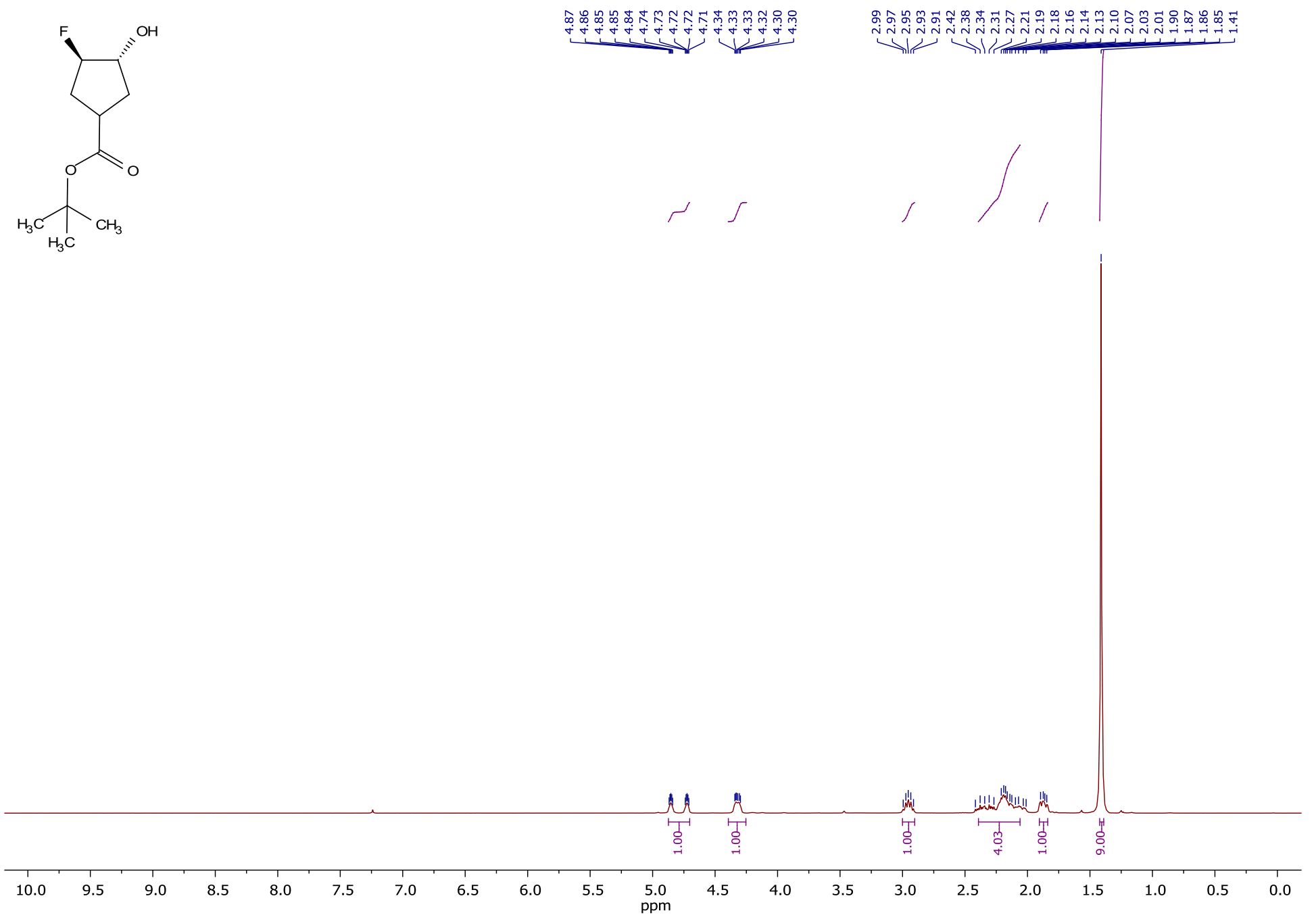

$\left(3 R^{*}, 4 R^{*}\right)$-tert-Butyl 3-fluoro-4-hydroxycyclopentanecarboxylate $(7 \mathrm{c}){ }^{1} \mathrm{H} \mathrm{NMR}\left(\mathrm{CDCl}_{3}\right)$ 

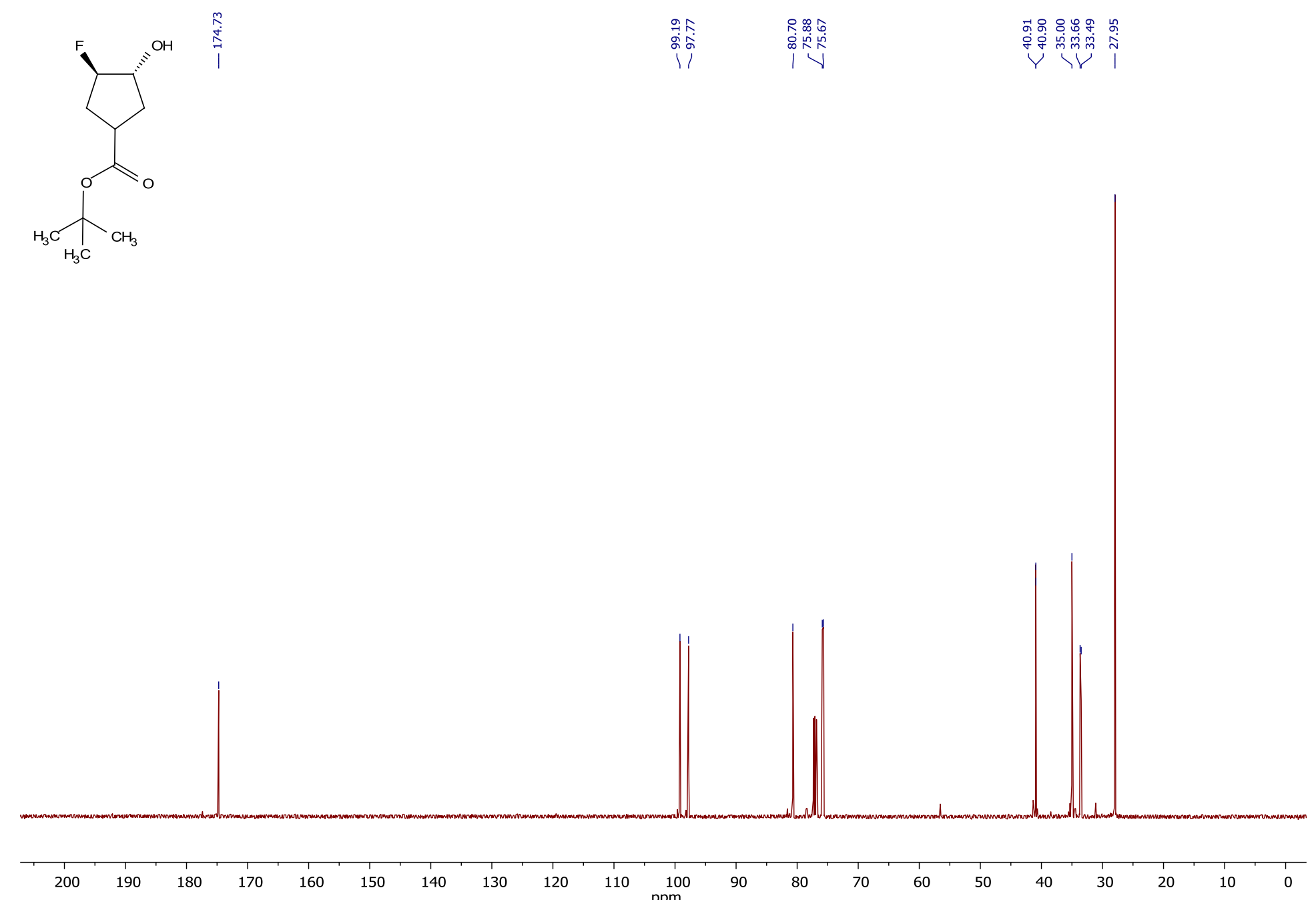

$\left(3 R^{*}, 4 R^{*}\right)$-tert-Butyl 3-fluoro-4-hydroxycyclopentanecarboxylate $(7 \mathrm{c}){ }^{13} \mathrm{C} \mathrm{NMR}\left(\mathrm{CDCl}_{3}\right)$ 


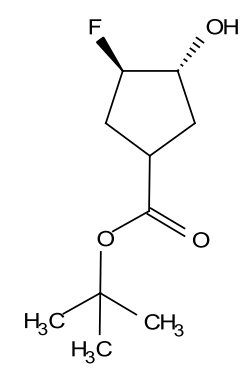

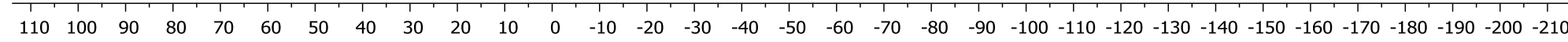
$\left(3 R^{*}, 4 R^{*}\right)$-tert-Butyl 3-fluoro-4-hydroxycyclopentanecarboxylate $(7 \mathrm{c}){ }^{19} \mathrm{~F} \mathrm{NMR}\left(\mathrm{CDCl}_{3}\right)$ 

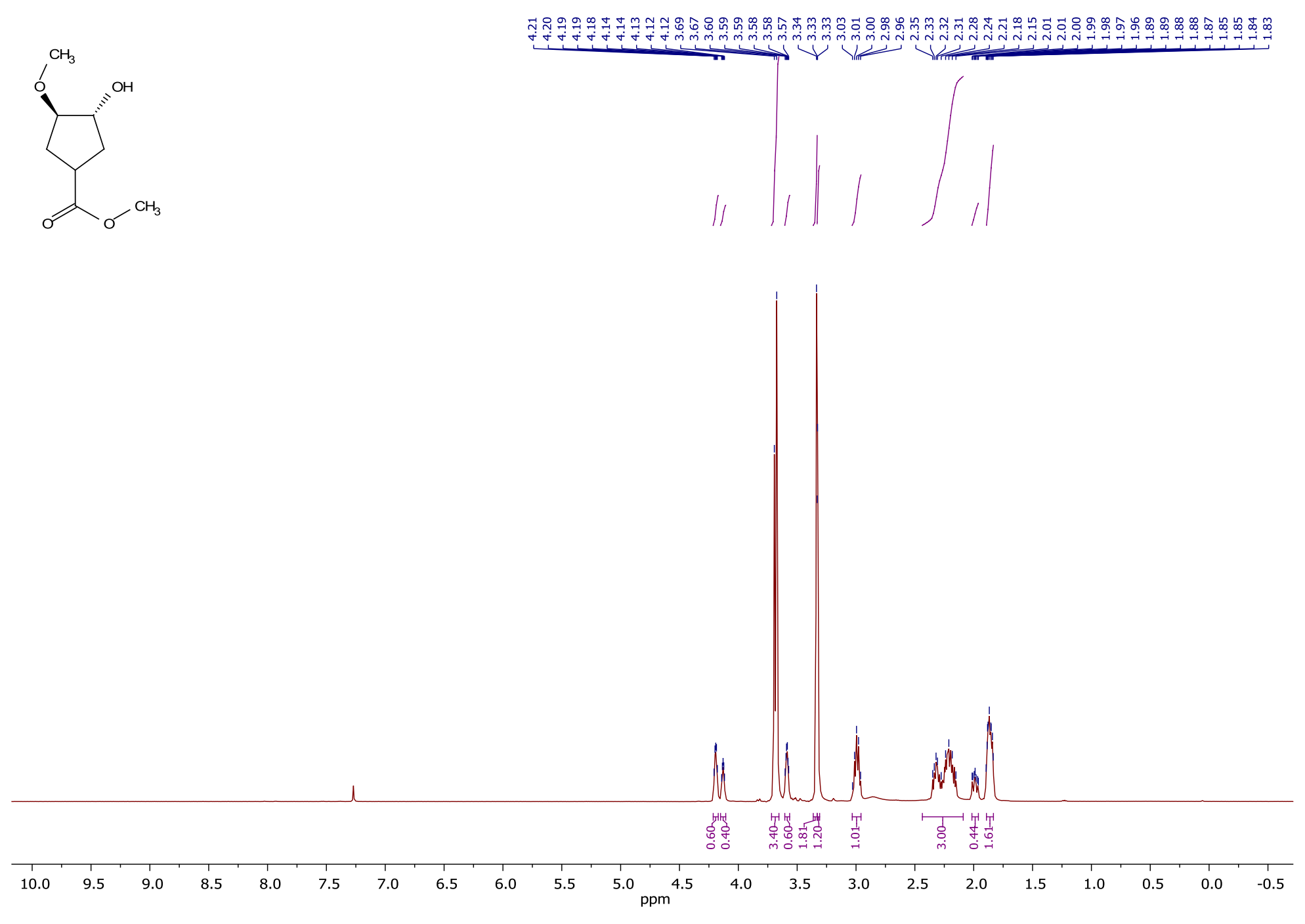

$\left(3 R^{*}, 4 R^{*}\right)$-Methyl 3-hydroxy-4-methoxycyclopentanecarboxylate (7d) ${ }^{1} \mathrm{H} \mathrm{NMR}\left(\mathrm{CDCl}_{3}\right)$ 

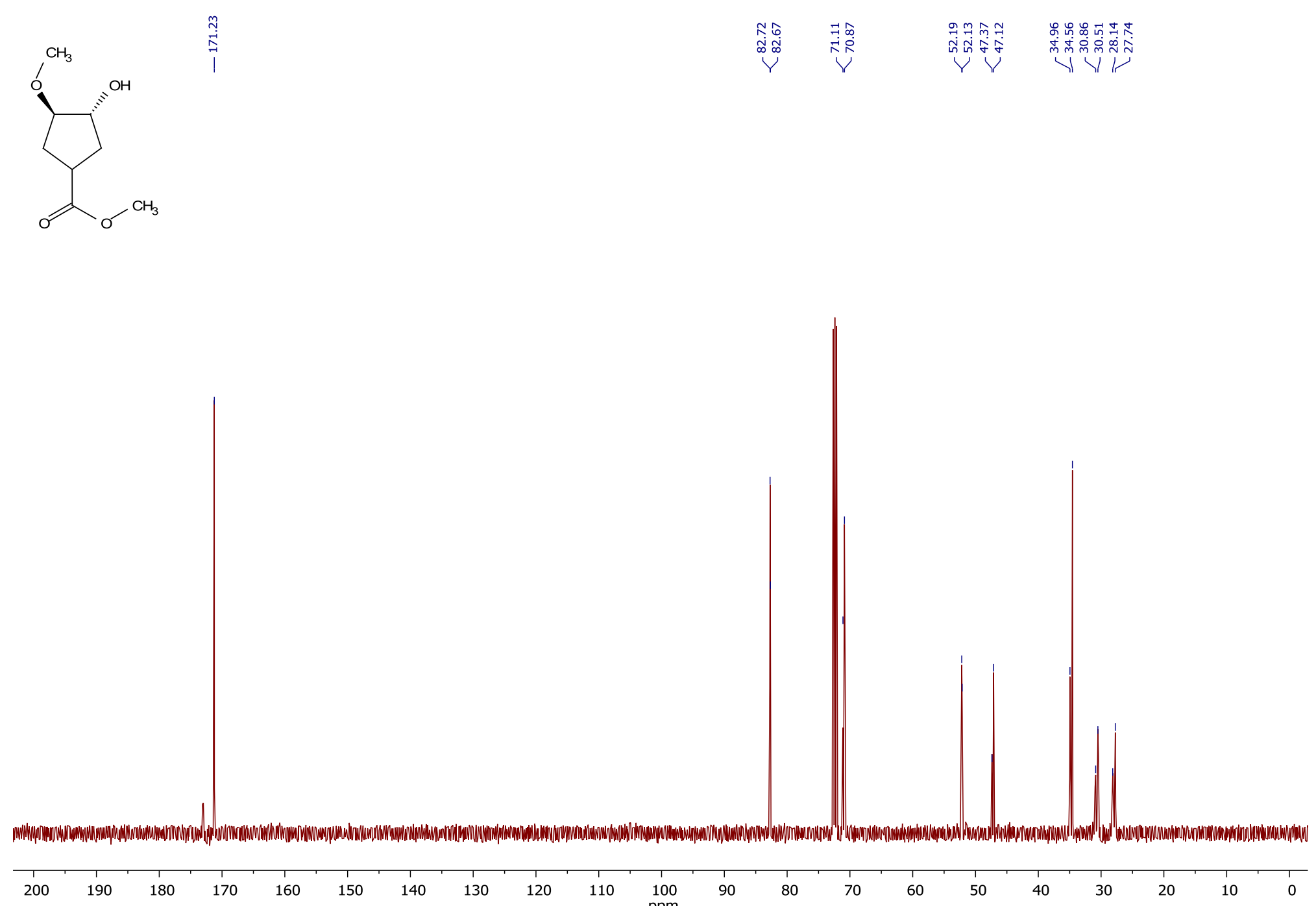

$\left(3 R^{*}, 4 R^{*}\right)$-Methyl 3-hydroxy-4-methoxycyclopentanecarboxylate (7d) ${ }^{13} \mathrm{C} \mathrm{NMR}\left(\mathrm{CDCl}_{3}\right)$ 


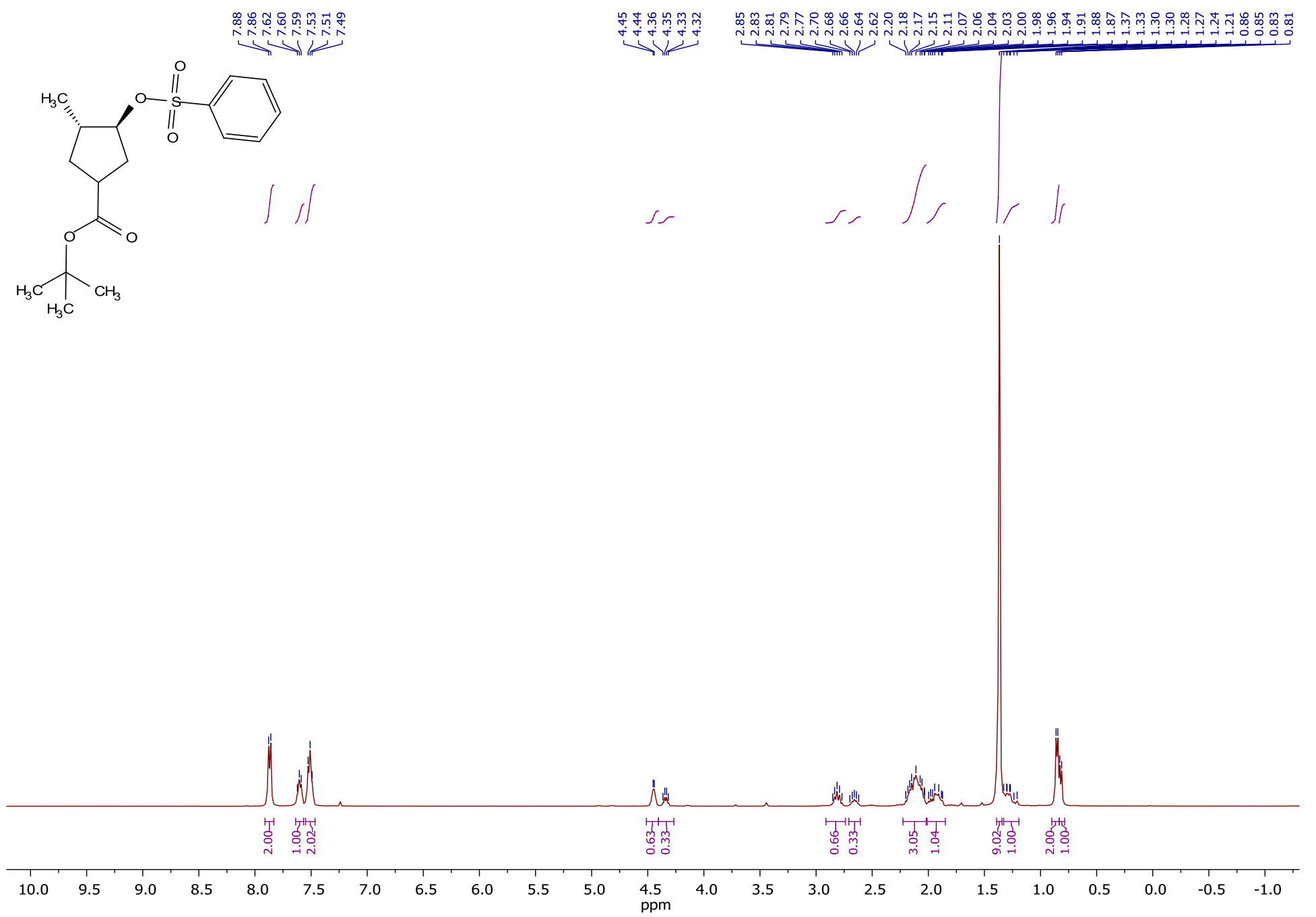

$\left(3 S^{*}, 4 S^{*}\right)$-tert-Butyl 3-methyl-4-((phenylsulfonyl)oxy)cyclopentanecarboxylate (8a) ${ }^{1} \mathrm{H}$ NMR $\left(\mathrm{CDCl}_{3}\right)$ 


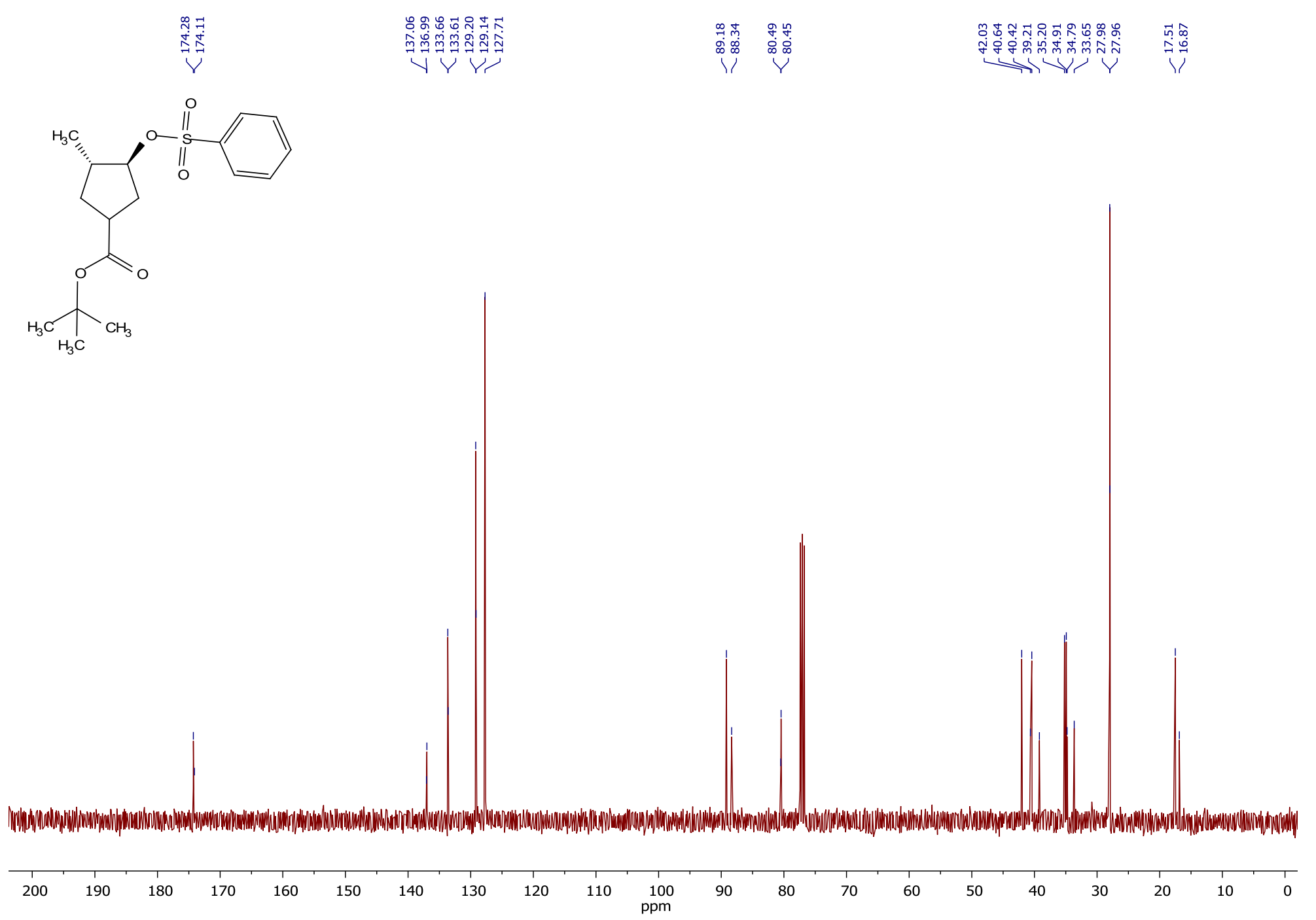

$\left(3 S^{*}, 4 S^{*}\right)$-tert-Butyl 3-methyl-4-((phenylsulfonyl)oxy)cyclopentanecarboxylate $(8 \mathrm{a}){ }^{13} \mathrm{C} \mathrm{NMR}\left(\mathrm{CDCl}_{3}\right)$ 


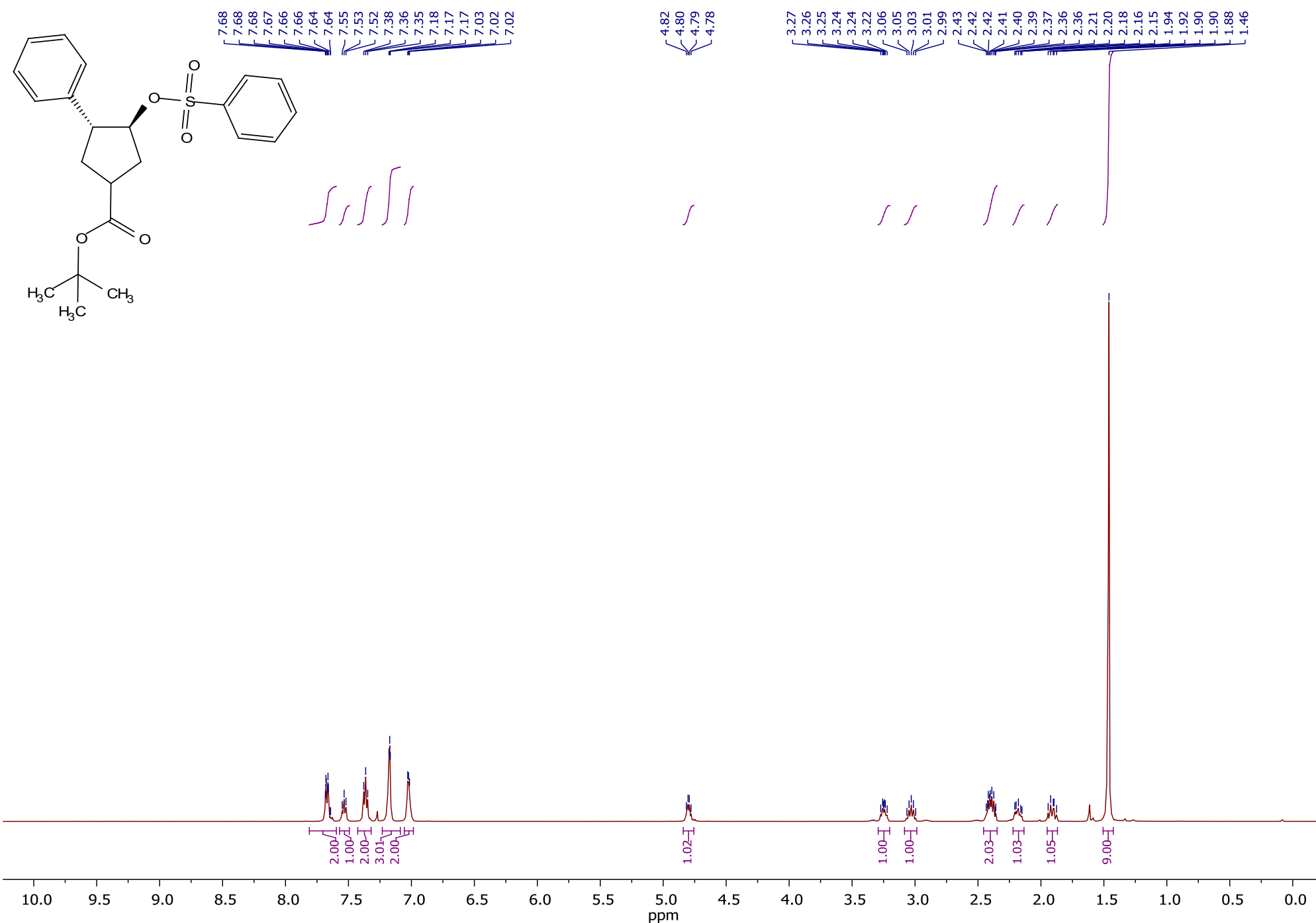

$\left(3 R^{*}, 4 S^{*}\right)$-tert-Butyl 3-phenyl-4-((phenylsulfonyl)oxy)cyclopentanecarboxylate (8b) ${ }^{1} \mathrm{H}$ NMR $\left(\mathrm{CDCl}_{3}\right)$ 


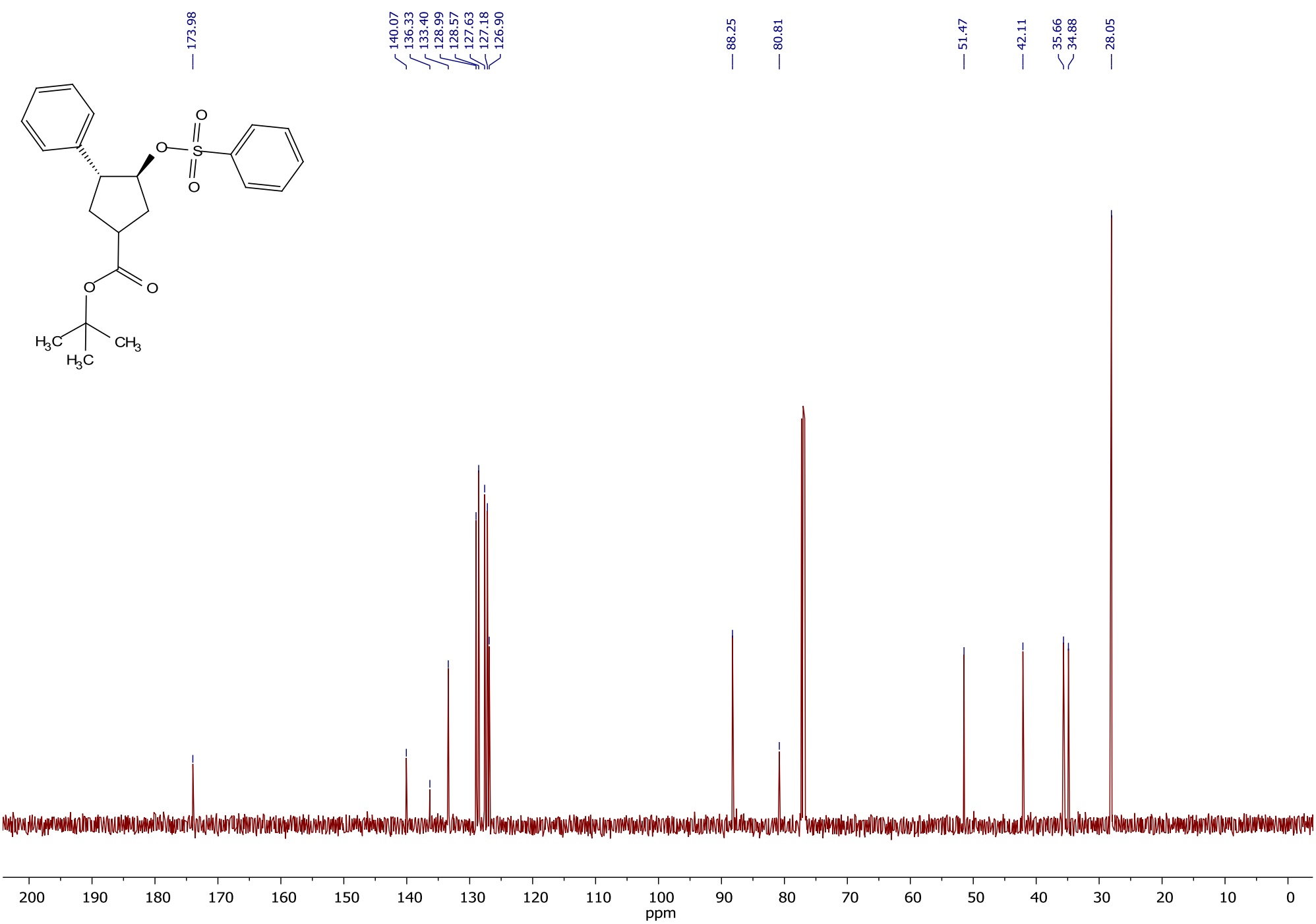

$\left(3 R^{*}, 4 S^{*}\right)$-tert-Butyl 3-phenyl-4-((phenylsulfonyl)oxy)cyclopentanecarboxylate $(8 \mathrm{~b}){ }^{13} \mathrm{C} \mathrm{NMR}\left(\mathrm{CDCl}_{3}\right)$ 

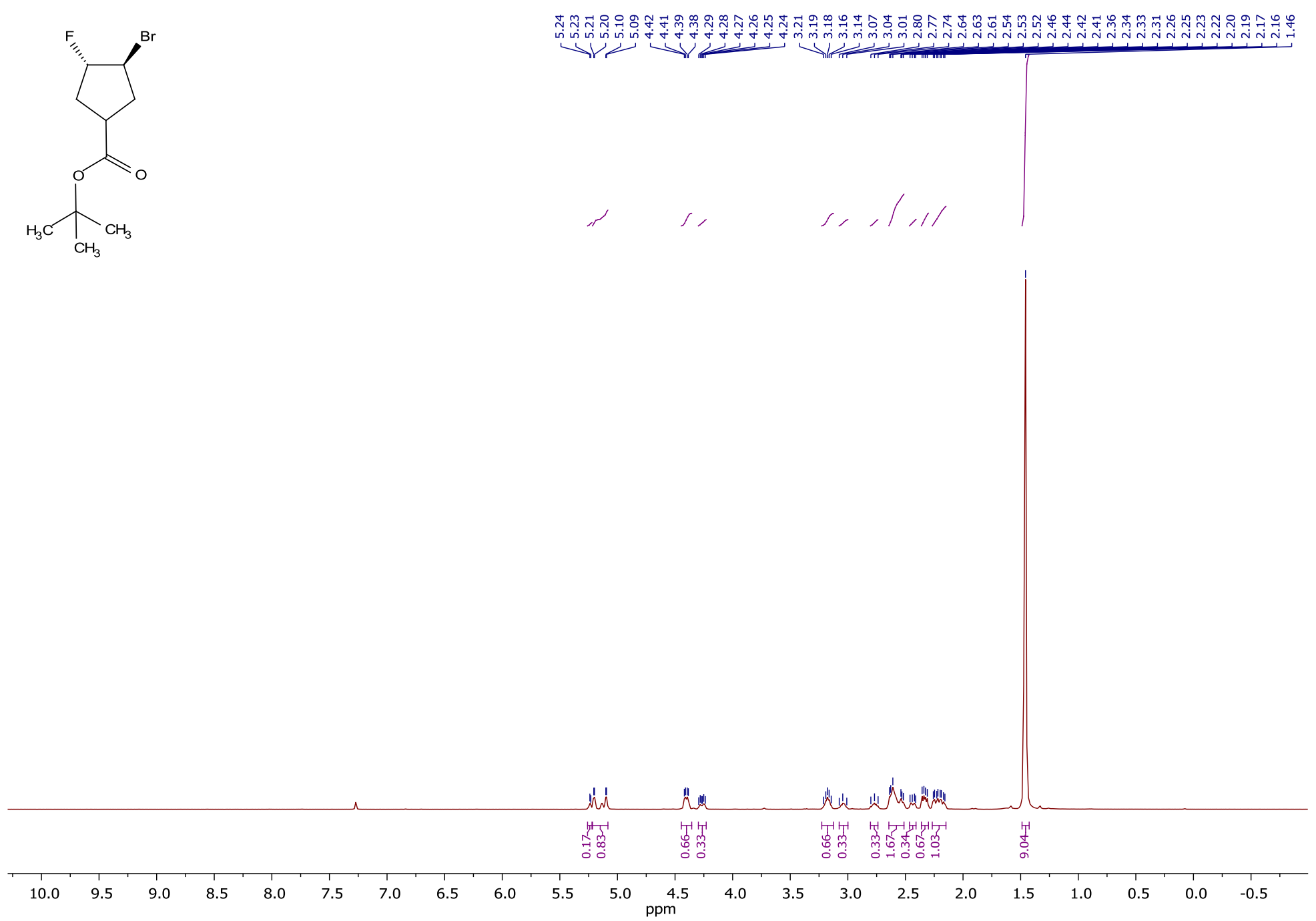

$\left(3 S^{*}, 4 S^{*}\right)$-tert-Butyl 3-bromo-4-fluorocyclopentanecarboxylate $(8 \mathrm{c}){ }^{1} \mathrm{H}$ NMR $\left(\mathrm{CDCl}_{3}\right)$ 

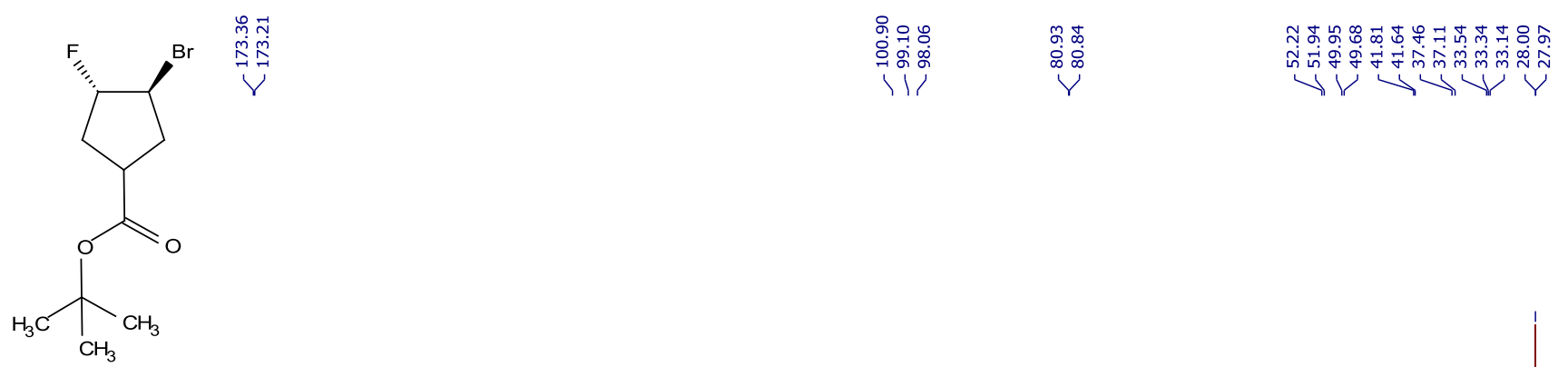

$\mathrm{CH}_{3}$

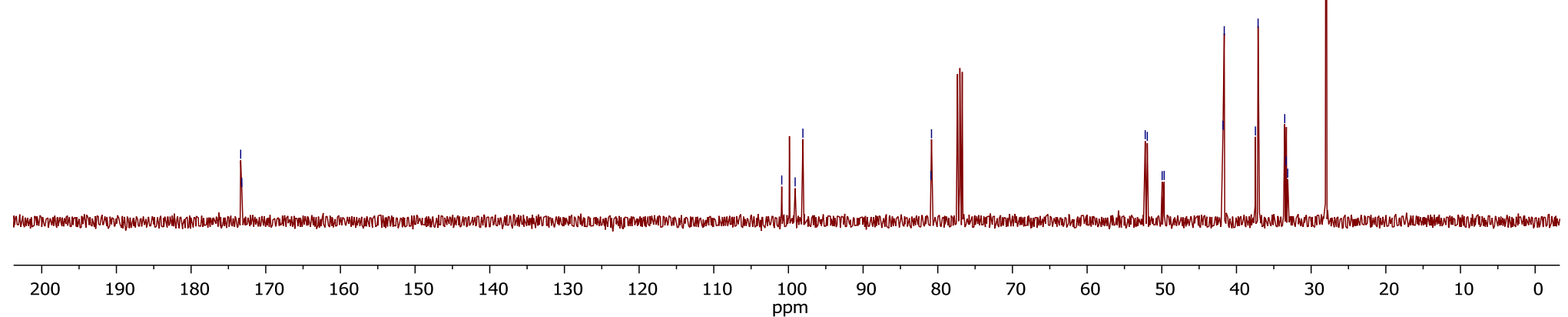

$\left(3 S^{*}, 4 S^{*}\right)$-tert-Butyl 3-bromo-4-fluorocyclopentanecarboxylate $(8 \mathrm{c}){ }^{13} \mathrm{C} \mathrm{NMR}\left(\mathrm{CDCl}_{3}\right)$ 

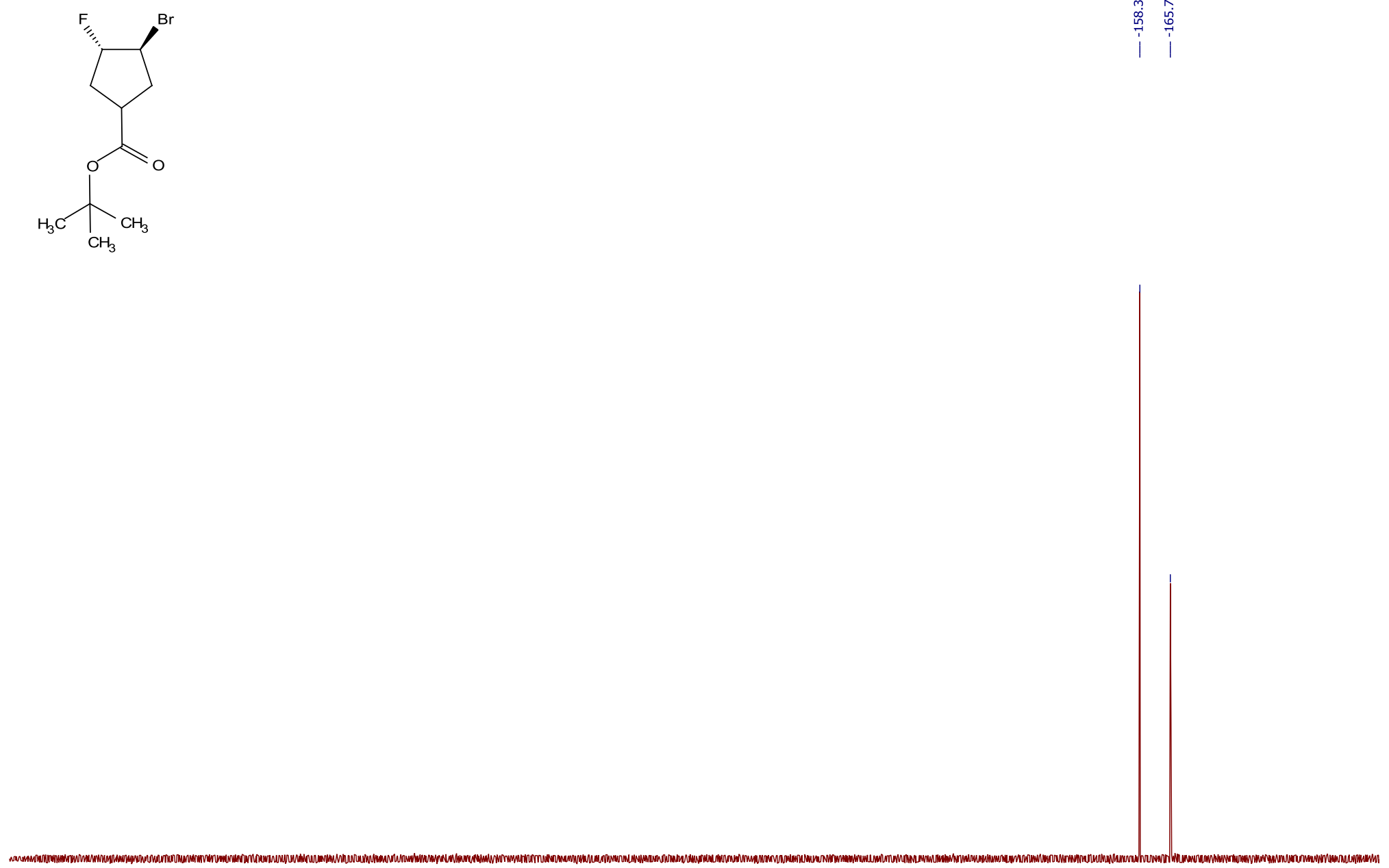

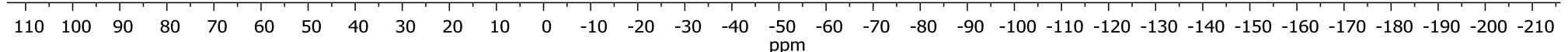
$\left(3 S^{*}, 4 S^{*}\right)$-tert-Butyl 3-bromo-4-fluorocyclopentanecarboxylate $(8 \mathrm{c}){ }^{19} \mathrm{~F}$ NMR $\left(\mathrm{CDCl}_{3}\right)$ 


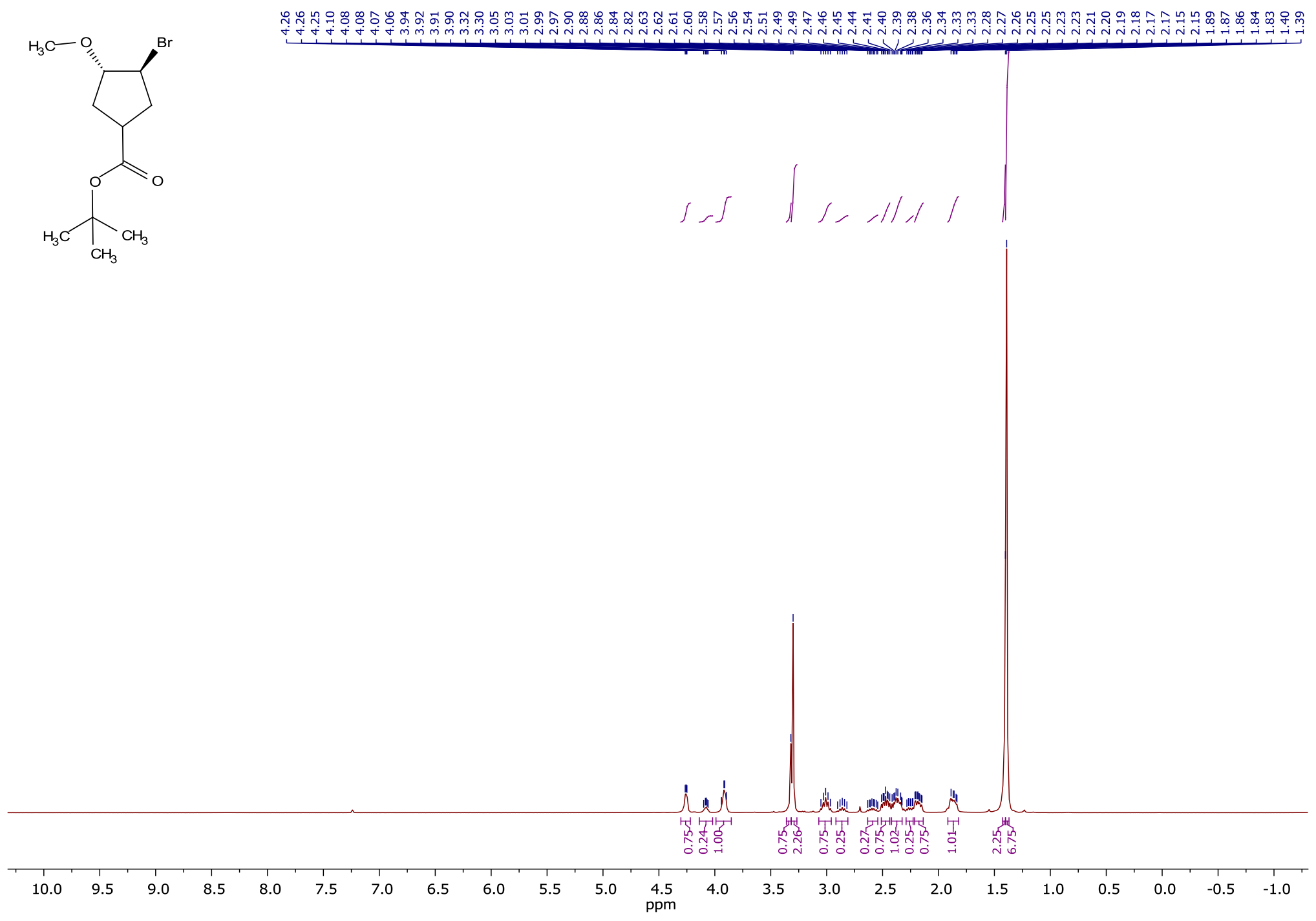

$\left(3 S^{*}, 4 S^{*}\right)$-tert-Butyl 3-bromo-4-methoxycyclopentanecarboxylate (8d) ${ }^{1} \mathrm{H} \mathrm{NMR}\left(\mathrm{CDCl}_{3}\right)$ 


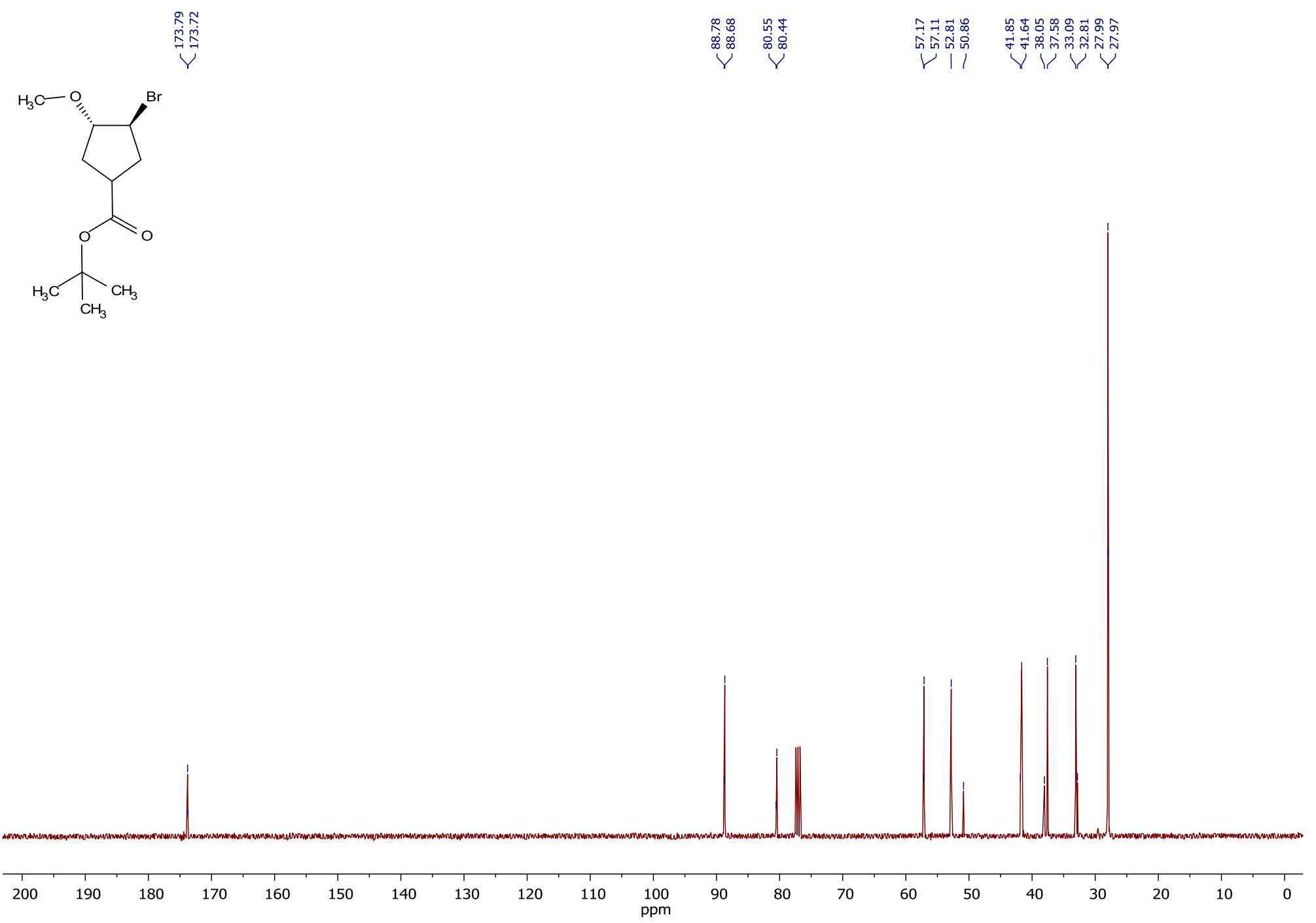

$\left(3 S^{*}, 4 S^{*}\right)$-tert-Butyl 3-bromo-4-methoxycyclopentanecarboxylate (8d) ${ }^{13} \mathrm{C} \mathrm{NMR}\left(\mathrm{CDCl}_{3}\right)$ 


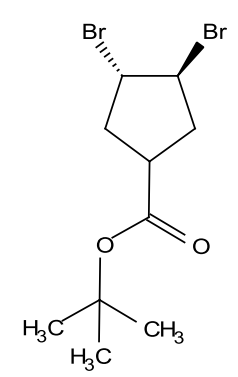

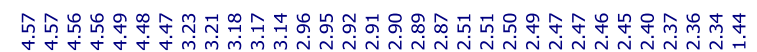

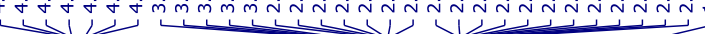

$\int 5$
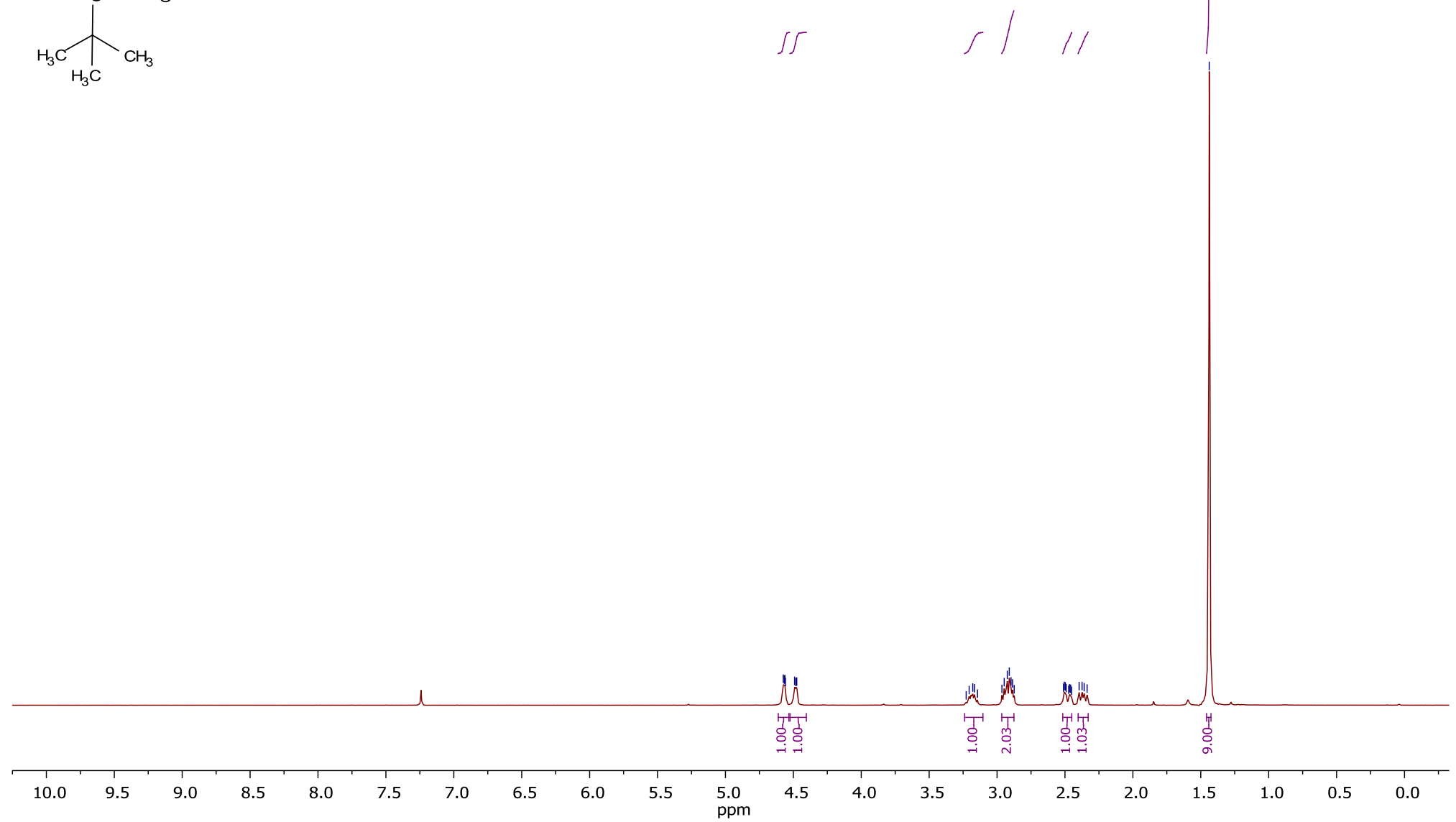

$\left(3 S^{*}, 4 S^{*}\right)$-tert-Butyl 3,4-dibromocyclopentanecarboxylate $(8 \mathrm{e}){ }^{1} \mathrm{H}^{\mathrm{NMR}}\left(\mathrm{CDCl}_{3}\right)$ 


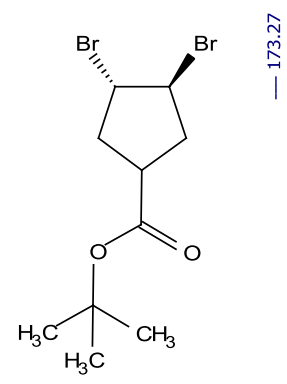

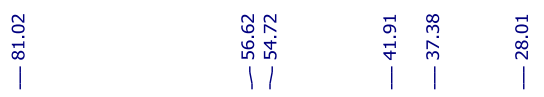

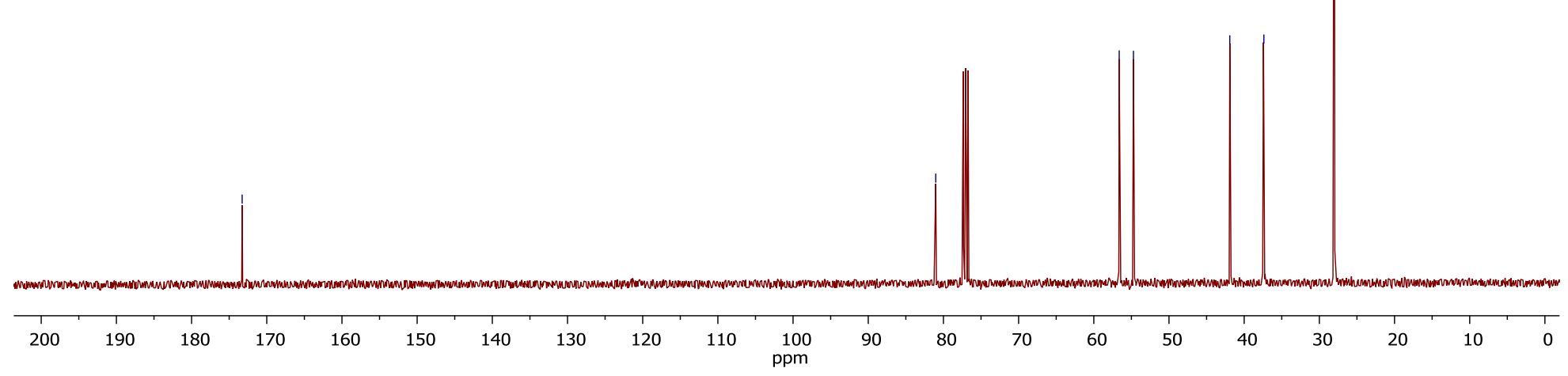

$\left(3 S^{*}, 4 S^{*}\right)$-tert-Butyl 3,4-dibromocyclopentanecarboxylate $(8 \mathrm{e}){ }^{13} \mathrm{C} \mathrm{NMR}\left(\mathrm{CDCl}_{3}\right)$ 


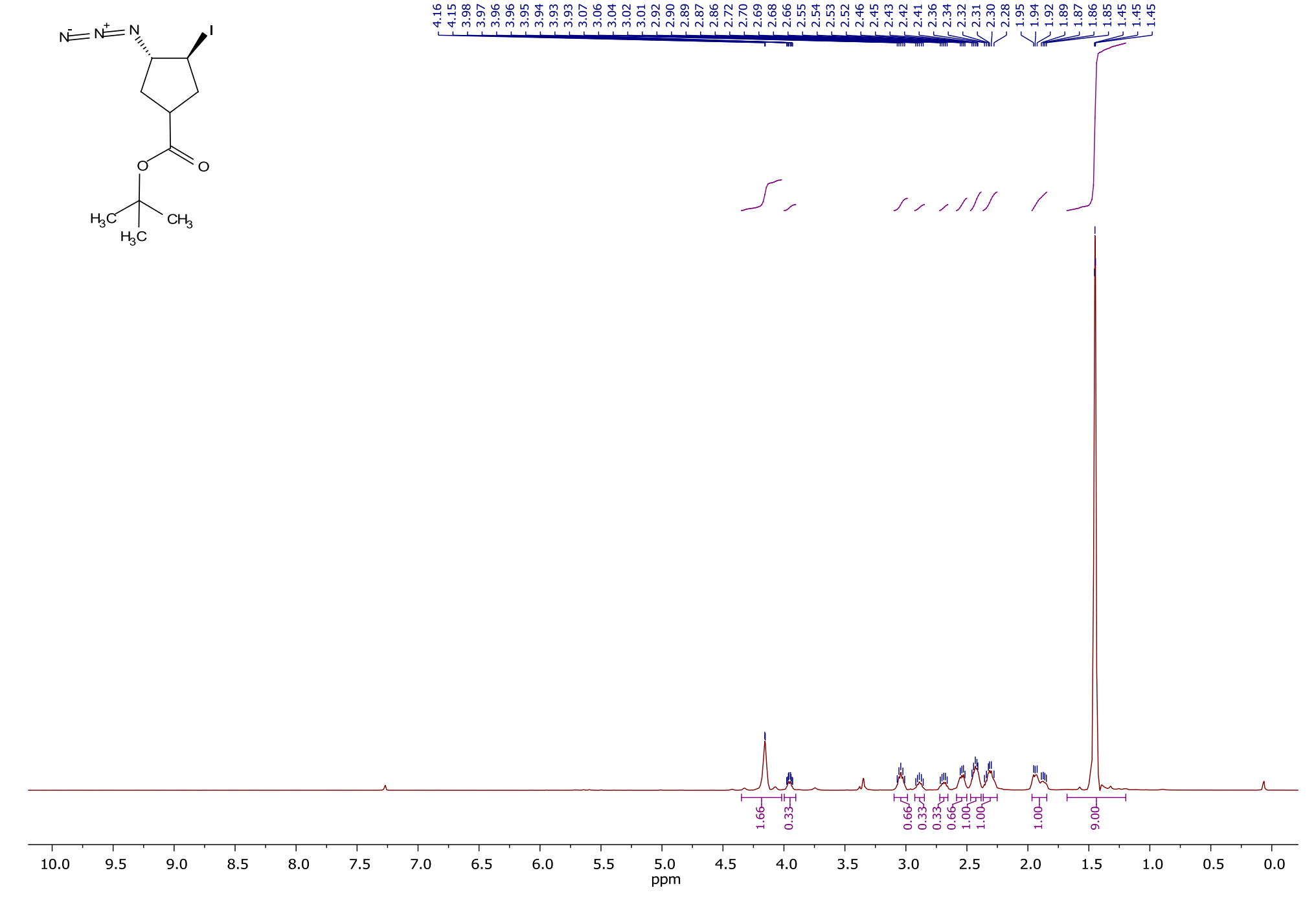

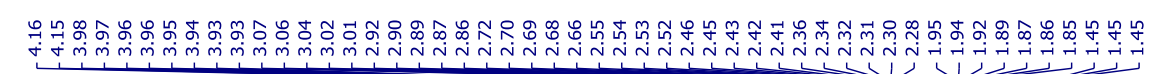

$\left(3 S^{*}, 4 S^{*}\right)$-tert-Butyl 3-azido-4-iodocyclopentanecarboxylate $(8 \mathrm{f}){ }^{1} \mathrm{H}$ NMR $\left(\mathrm{CDCl}_{3}\right)$ 


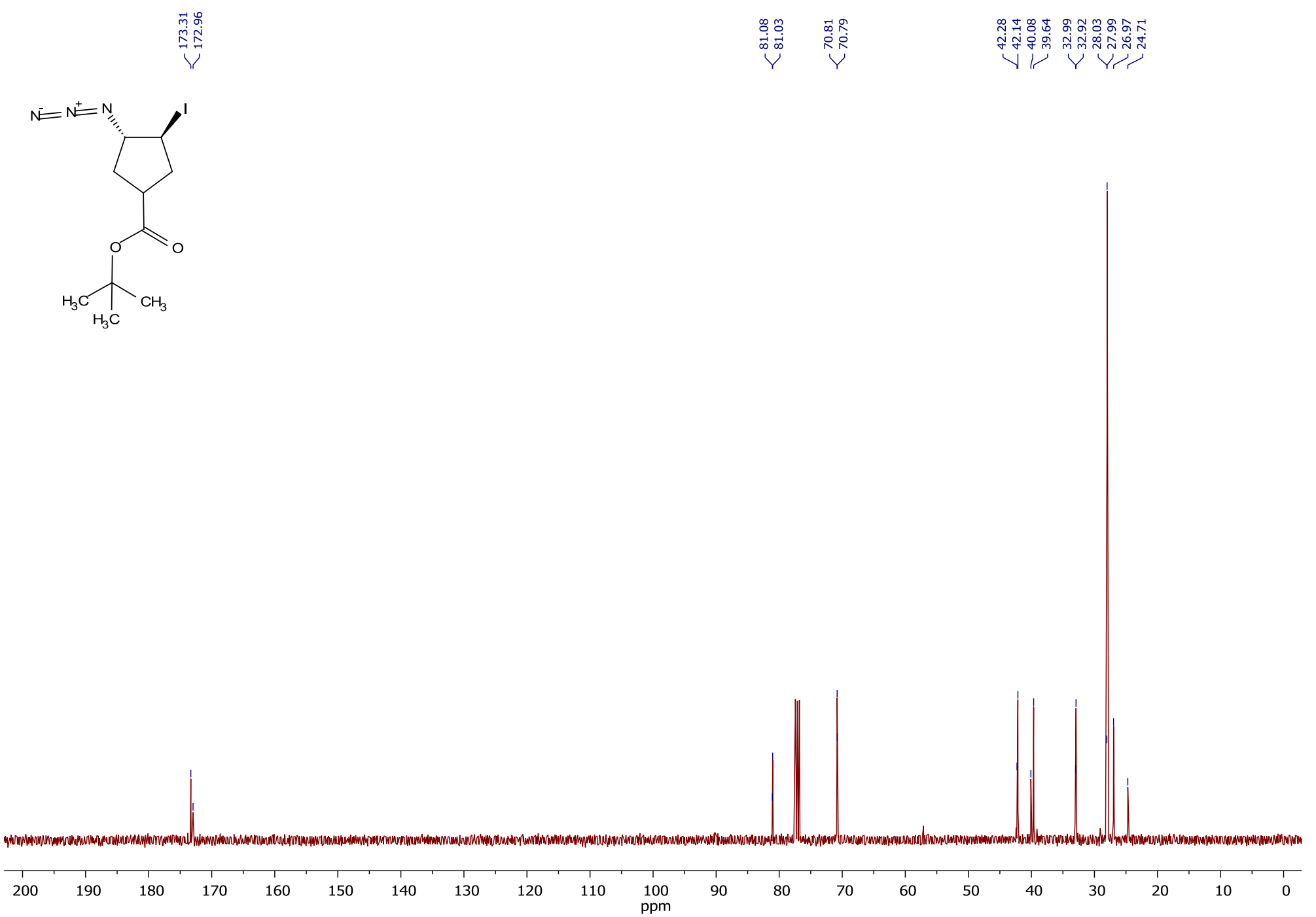

$\left(3 S^{*}, 4 S^{*}\right)$-tert-Butyl 3-azido-4-iodocyclopentanecarboxylate (8f) ${ }^{13} \mathrm{C} \mathrm{NMR}\left(\mathrm{CDCl}_{3}\right)$ 


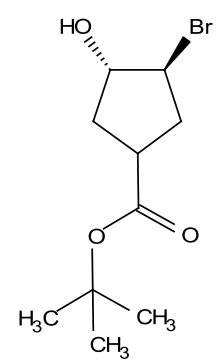

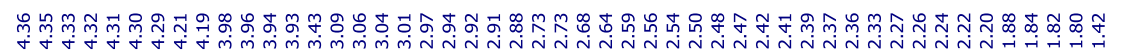

$\mathrm{CH}_{3}$
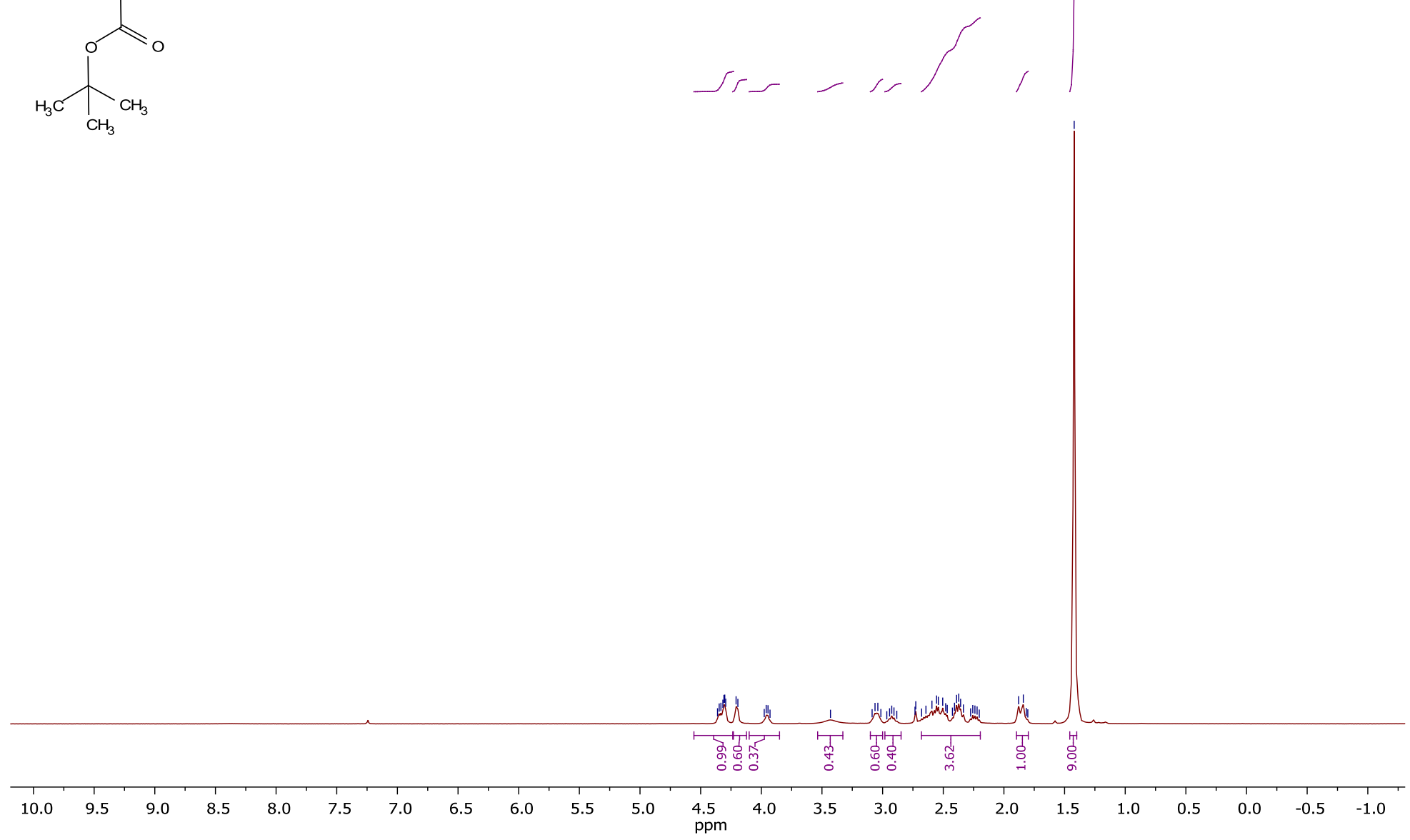

$\left(3 S^{*}, 4 S^{*}\right)$-tert-Butyl 3-bromo-4-hydroxycyclopentanecarboxylate $(8 \mathrm{~g}){ }^{1} \mathrm{H} \mathrm{NMR}\left(\mathrm{CDCl}_{3}\right)$ 


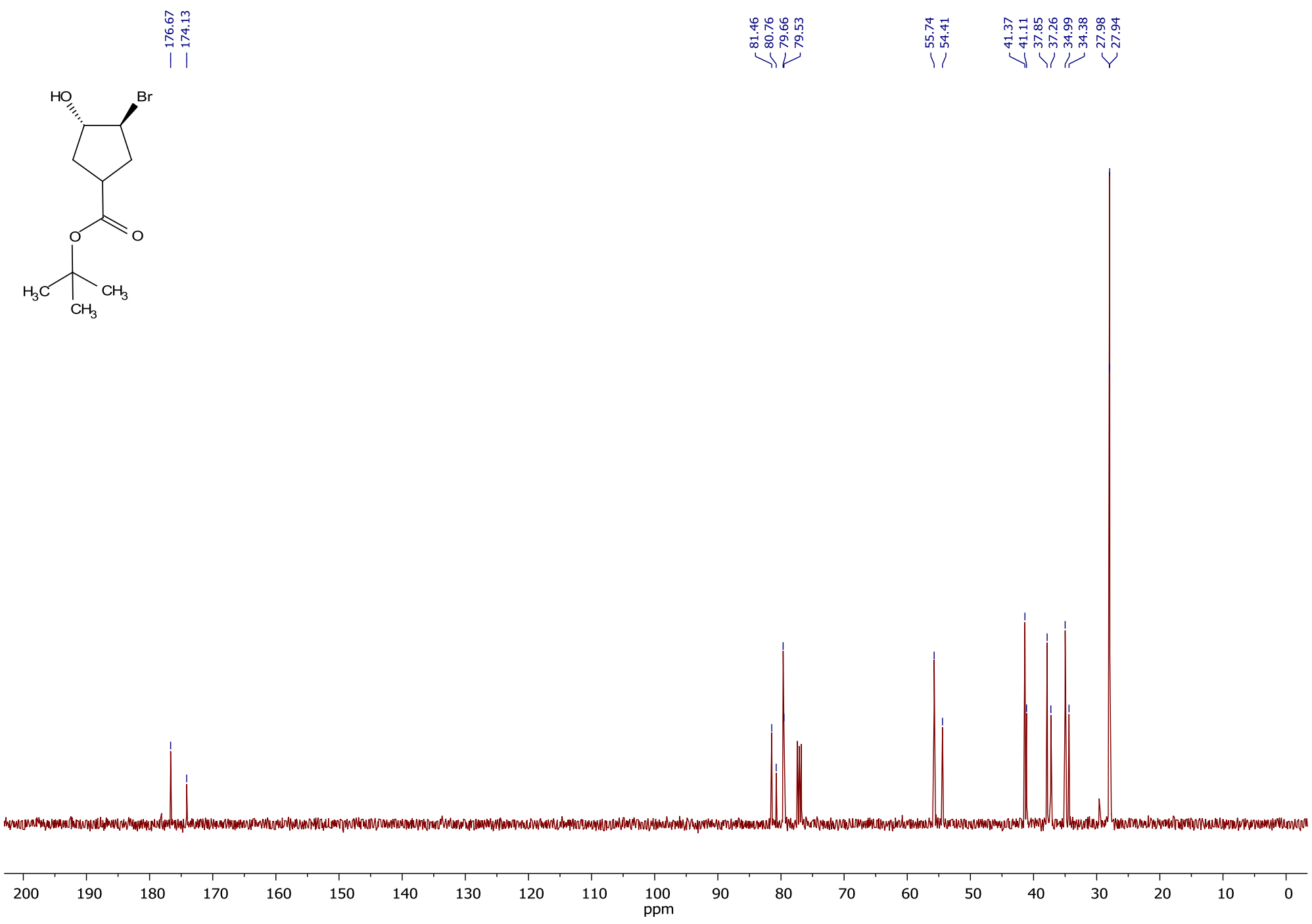

$\left(3 S^{*}, 4 S^{*}\right)$-tert-Butyl 3-bromo-4-hydroxycyclopentanecarboxylate $(8 \mathrm{~g}){ }^{13} \mathrm{C} \mathrm{NMR}\left(\mathrm{CDCl}_{3}\right)$ 

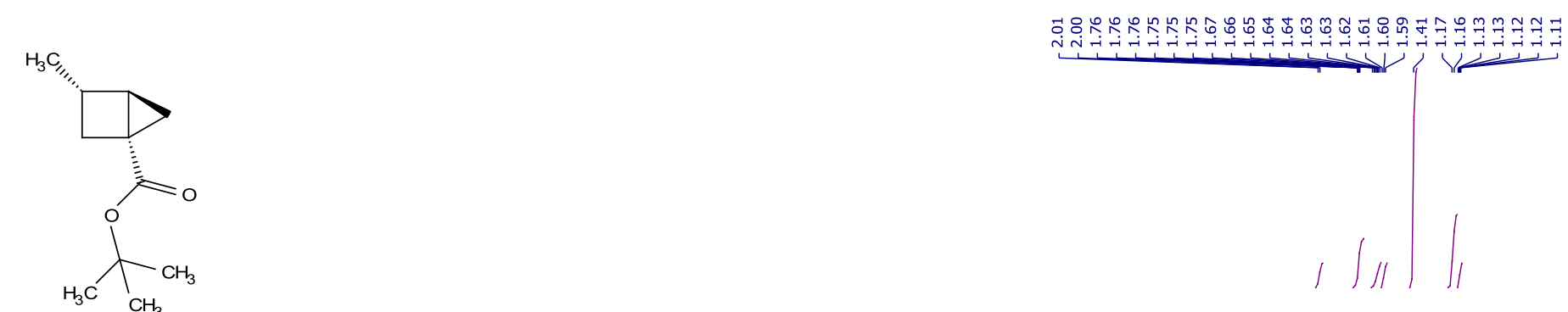

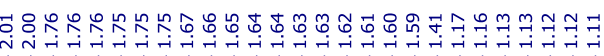

$\mathrm{CH}_{3}$
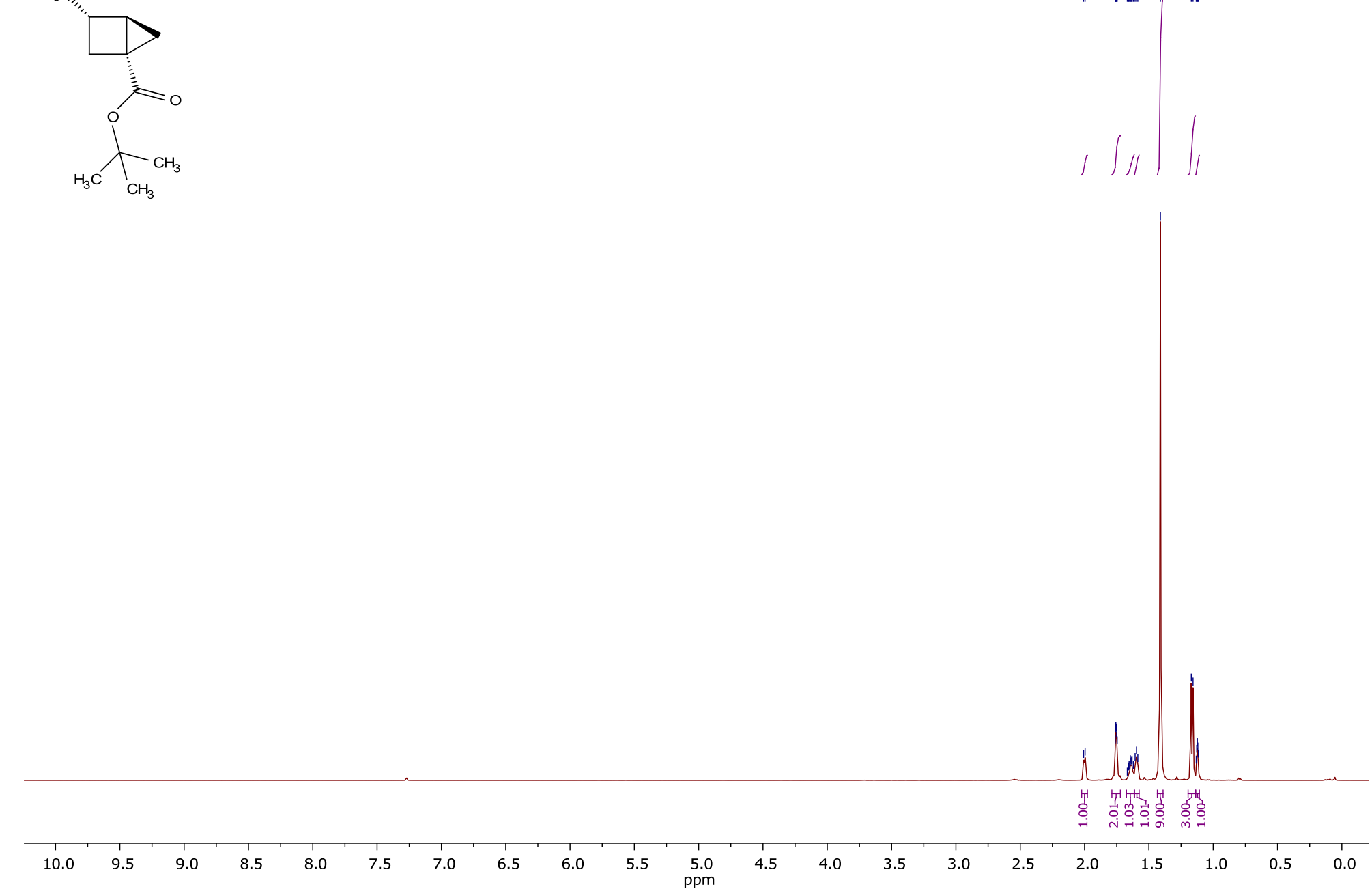

$\left(1 R^{*}, 3 S^{*}, 4 R^{*}\right)$-tert-Butyl 3-methylbicyclo[2.1.0]pentane-1-carboxylate (9a) ${ }^{1} \mathrm{H}$ NMR $\left(\mathrm{CDCl}_{3}\right)$ 


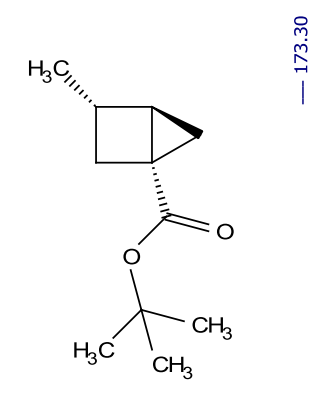

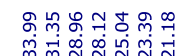

(1)

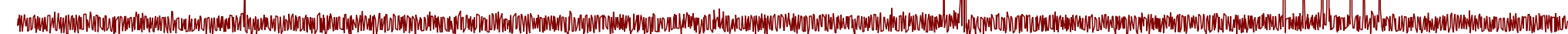




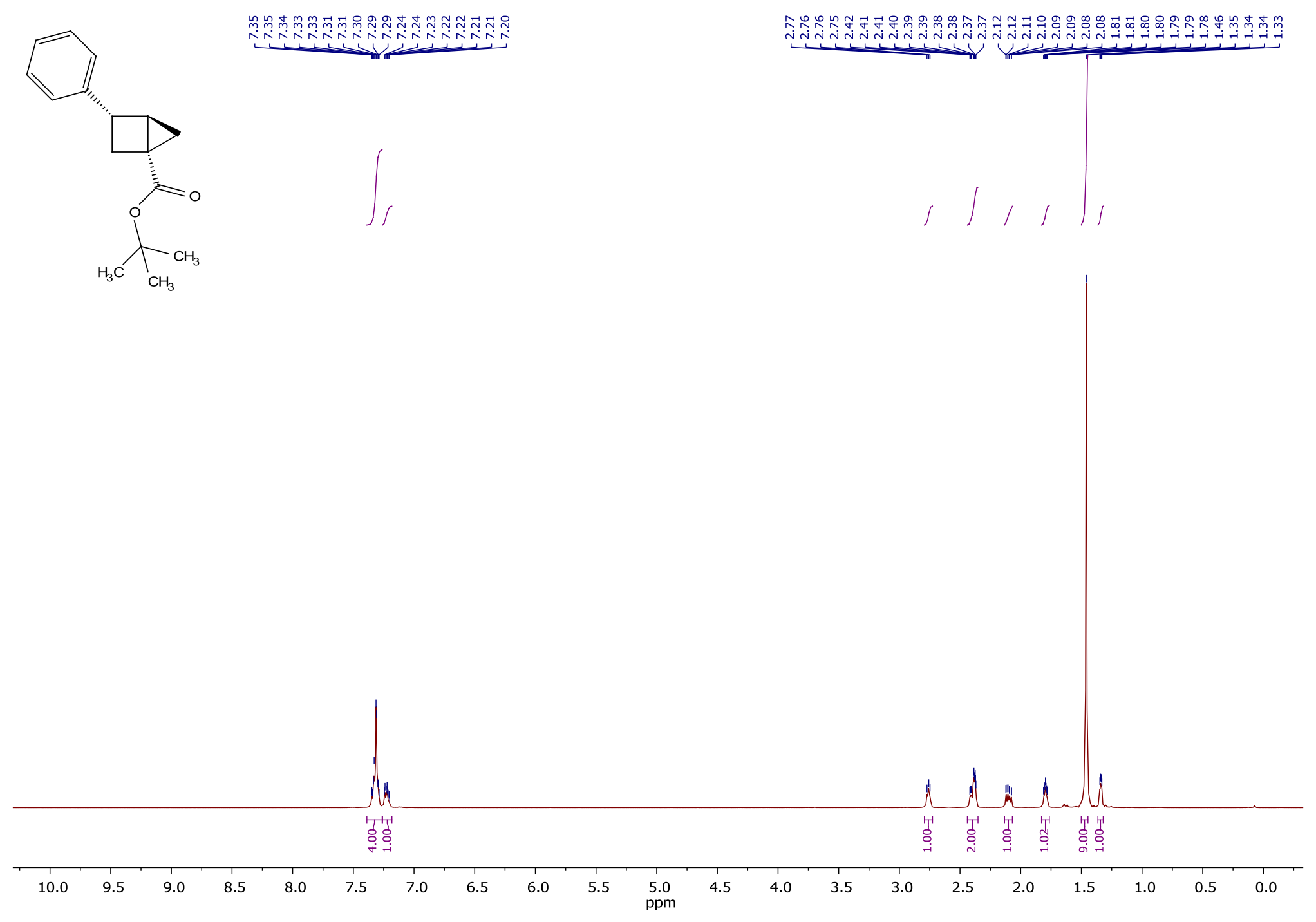

$\left(1 R^{*}, 3 S^{*}, 4 R^{*}\right)$-tert-Butyl 3-phenylbicyclo[2.1.0]pentane-1-carboxylate (9b) ${ }^{1} \mathrm{H}$ NMR $\left(\mathrm{CDCl}_{3}\right)$ 


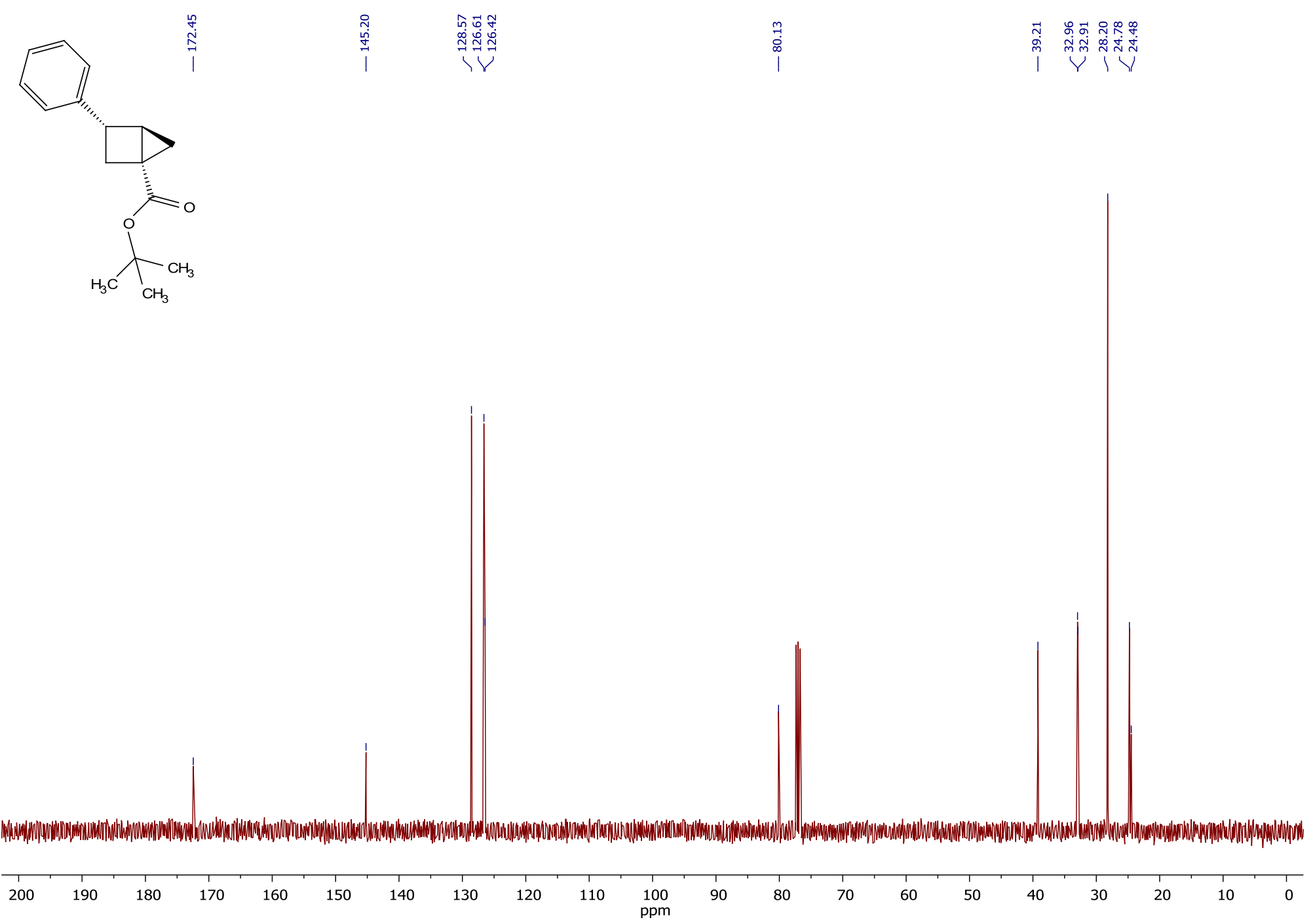

$\left(1 R^{*}, 3 S^{*}, 4 R^{*}\right)$-tert-Butyl 3-phenylbicyclo[2.1.0]pentane-1-carboxylate (9b) ${ }^{13} \mathrm{C} \mathrm{NMR}\left(\mathrm{CDCl}_{3}\right)$ 

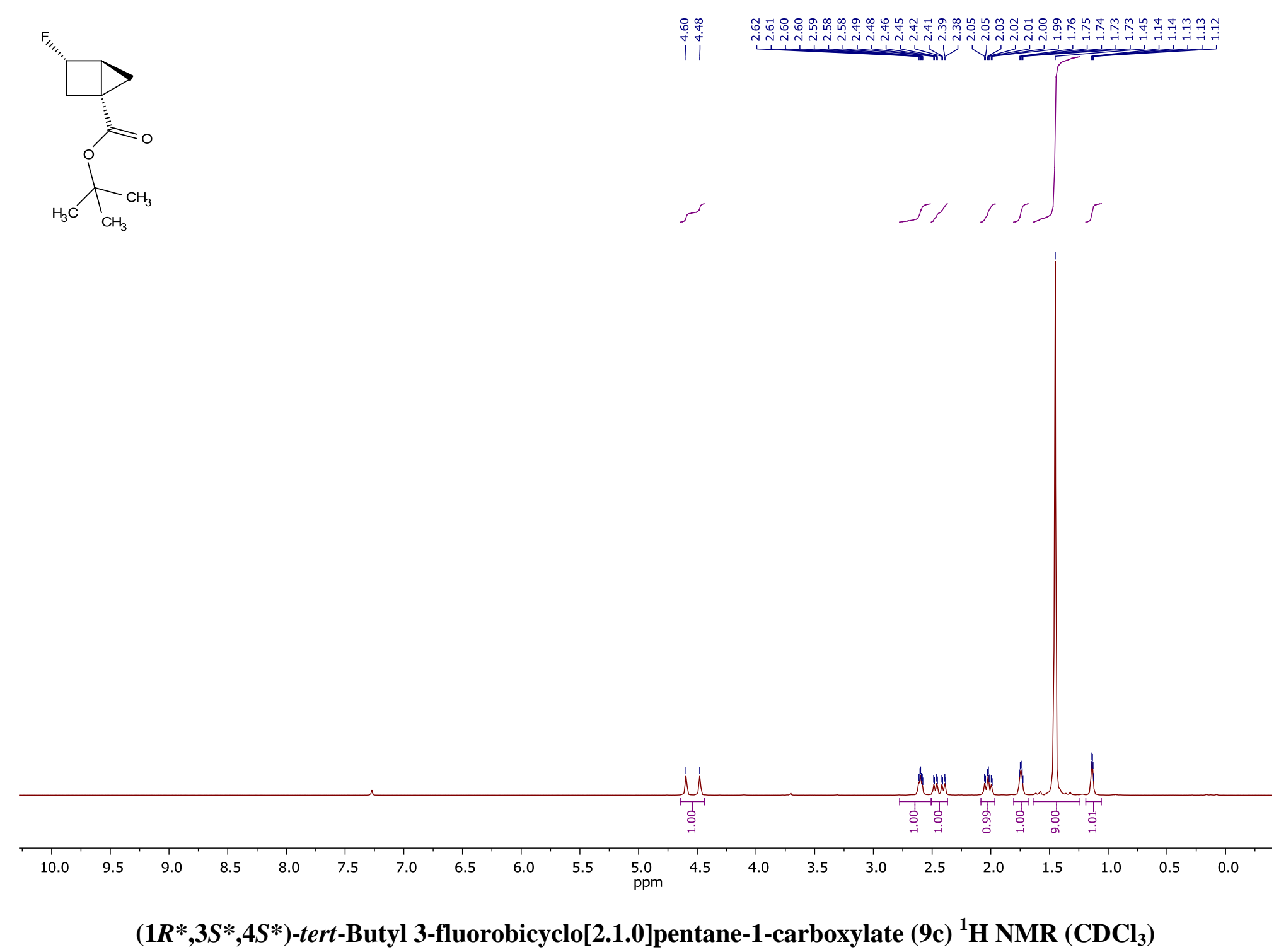

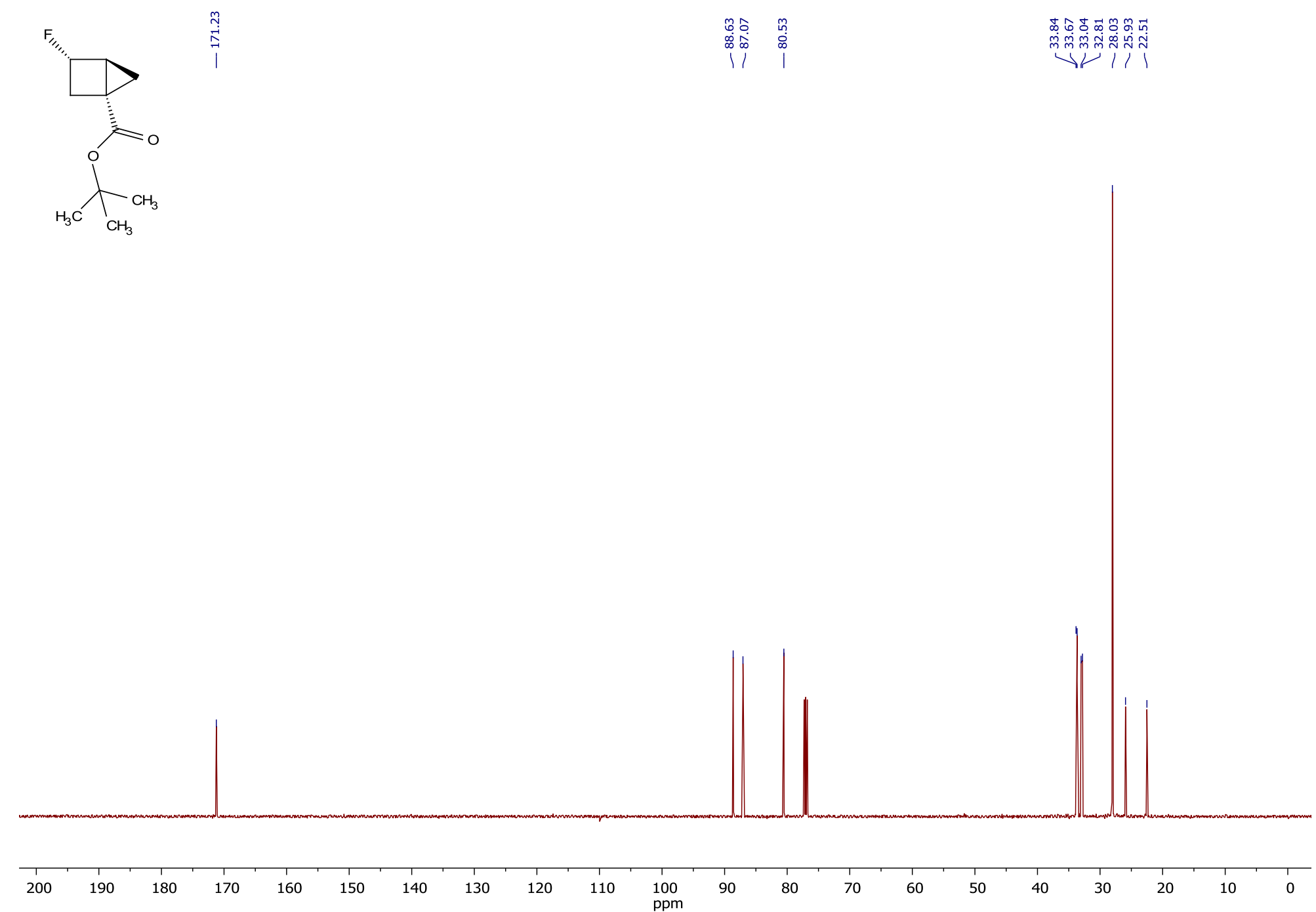

$\left(1 R^{*}, 3 S^{*}, 4 S^{*}\right)$-tert-Butyl 3-fluorobicyclo[2.1.0]pentane-1-carboxylate (9c) ${ }^{13} \mathrm{C}$ NMR $\left(\mathrm{CDCl}_{3}\right)$ 

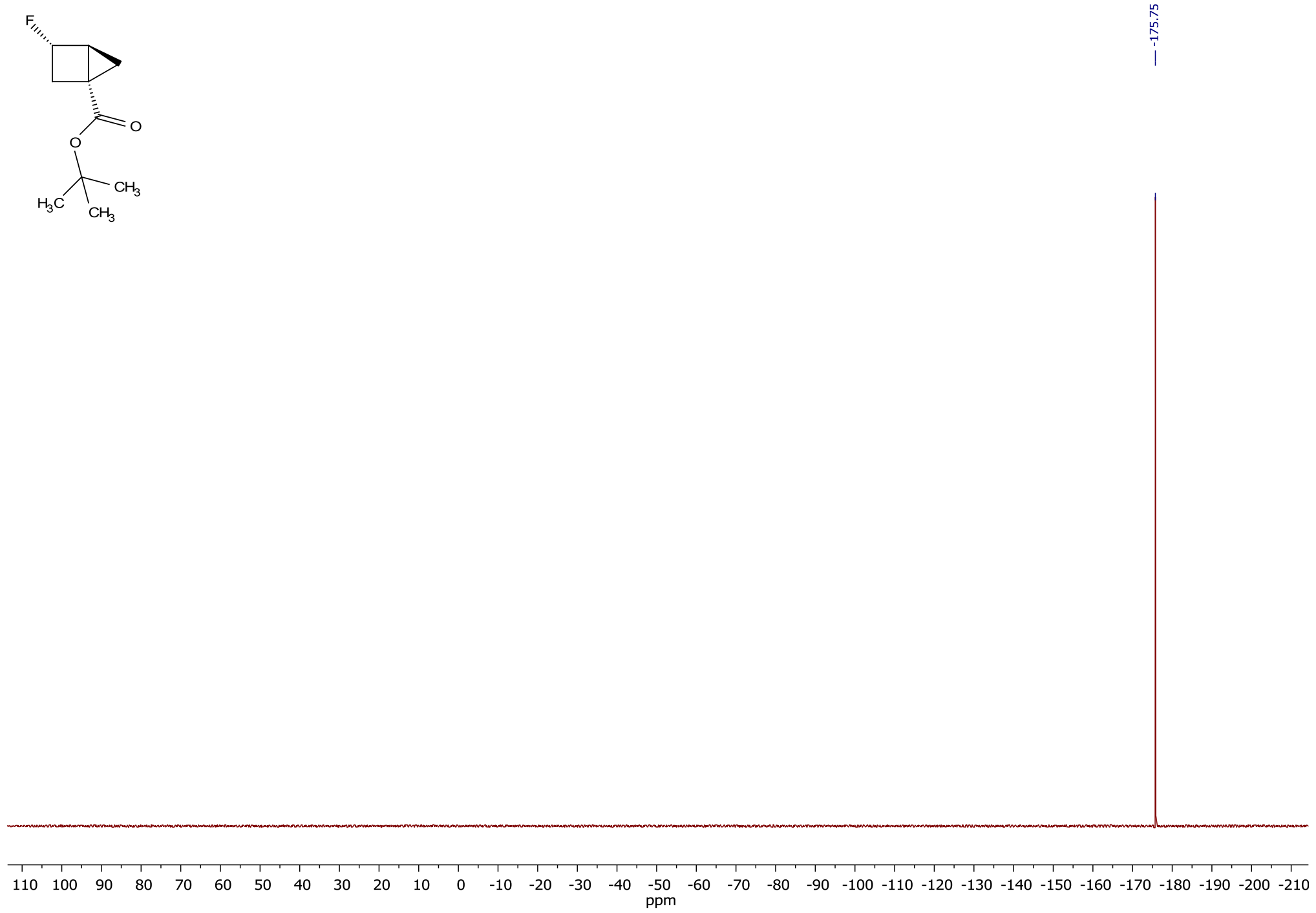

$\left(1 R^{*}, 3 S^{*}, 4 S^{*}\right)$-tert-Butyl 3-fluorobicyclo[2.1.0]pentane-1-carboxylate $(9 \mathrm{c}){ }^{19} \mathrm{~F} \mathrm{NMR}\left(\mathrm{CDCl}_{3}\right)$ 

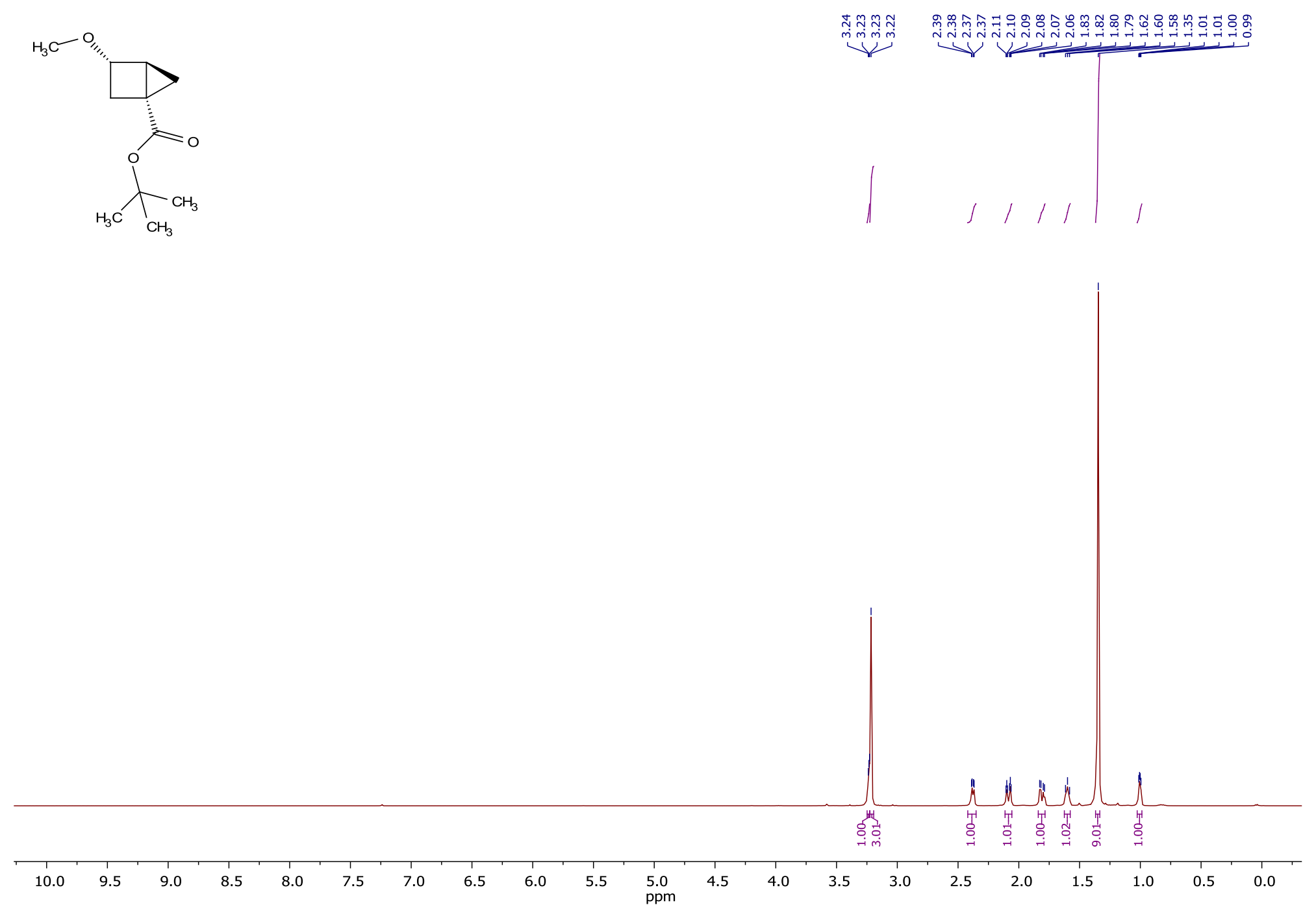

$\left(1 R^{*}, 3 S^{*}, 4 S^{*}\right)$-tert-Butyl 3-methoxybicyclo[2.1.0]pentane-1-carboxylate (9d) ${ }^{1} \mathrm{H}$ NMR $\left(\mathrm{CDCl}_{3}\right)$ 

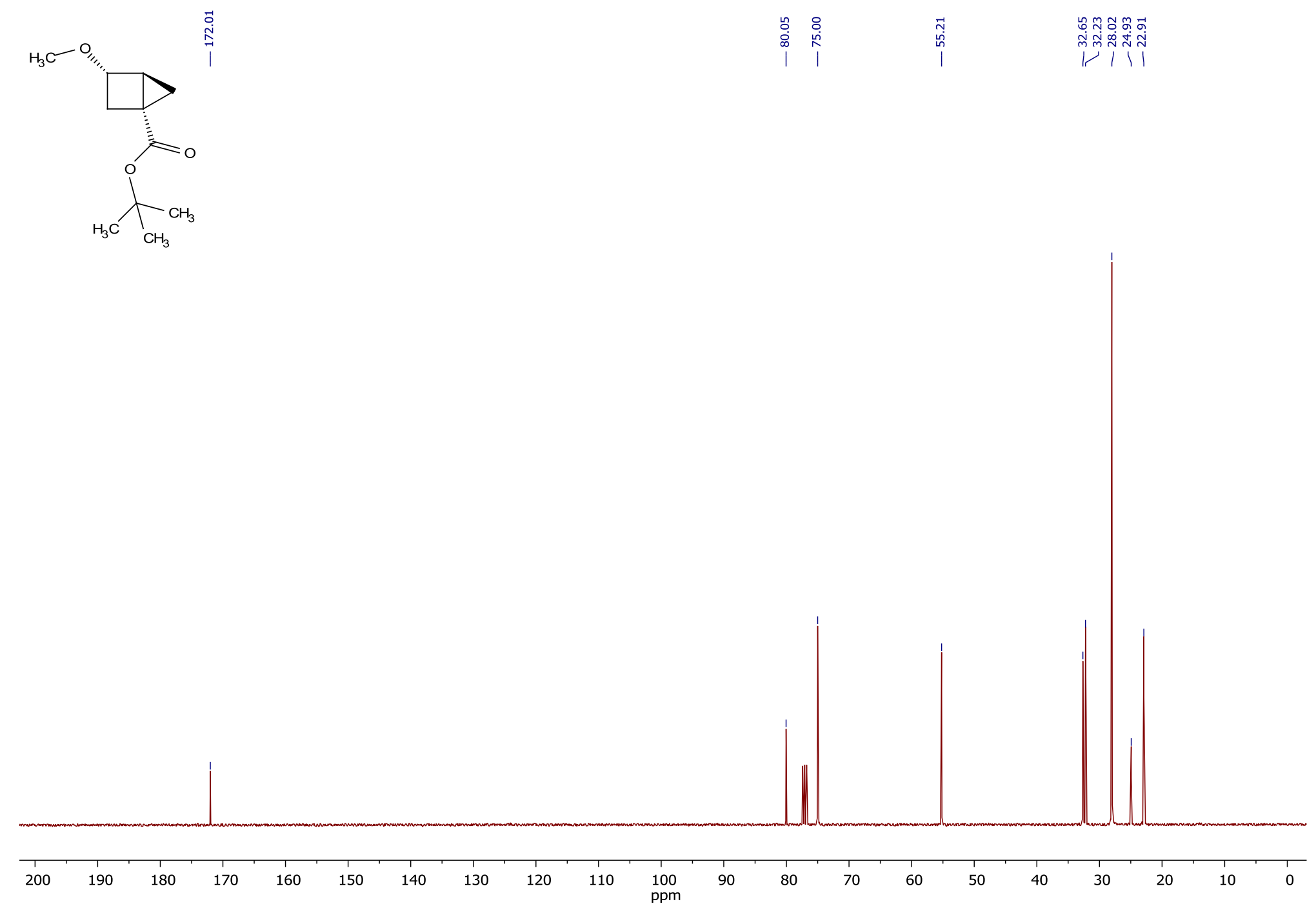

$\left(1 R^{*}, 3 S^{*}, 4 S^{*}\right)$-tert-Butyl 3-methoxybicyclo[2.1.0]pentane-1-carboxylate (9d) ${ }^{13} \mathrm{C}$ NMR $\left(\mathrm{CDCl}_{3}\right)$ 

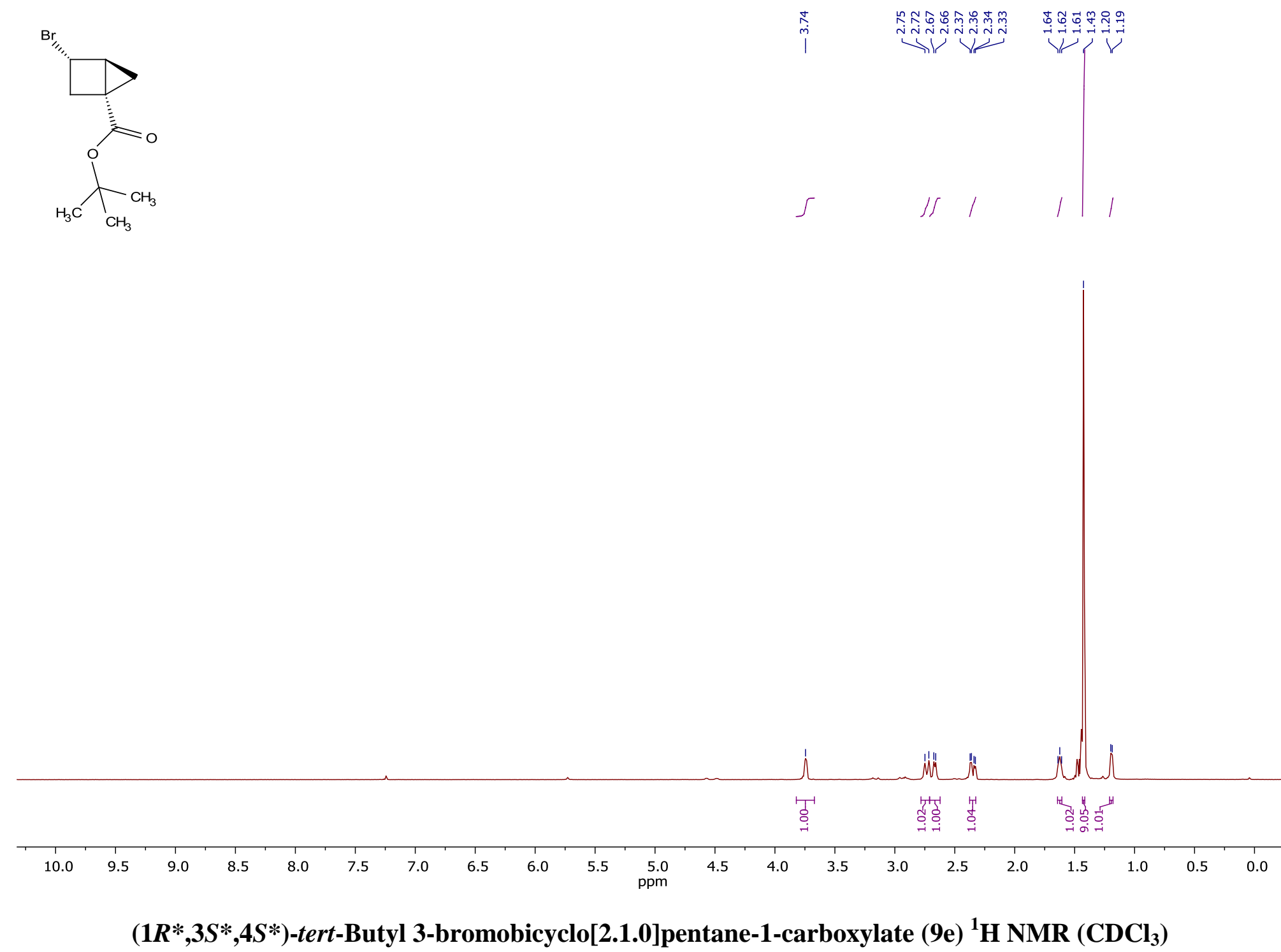

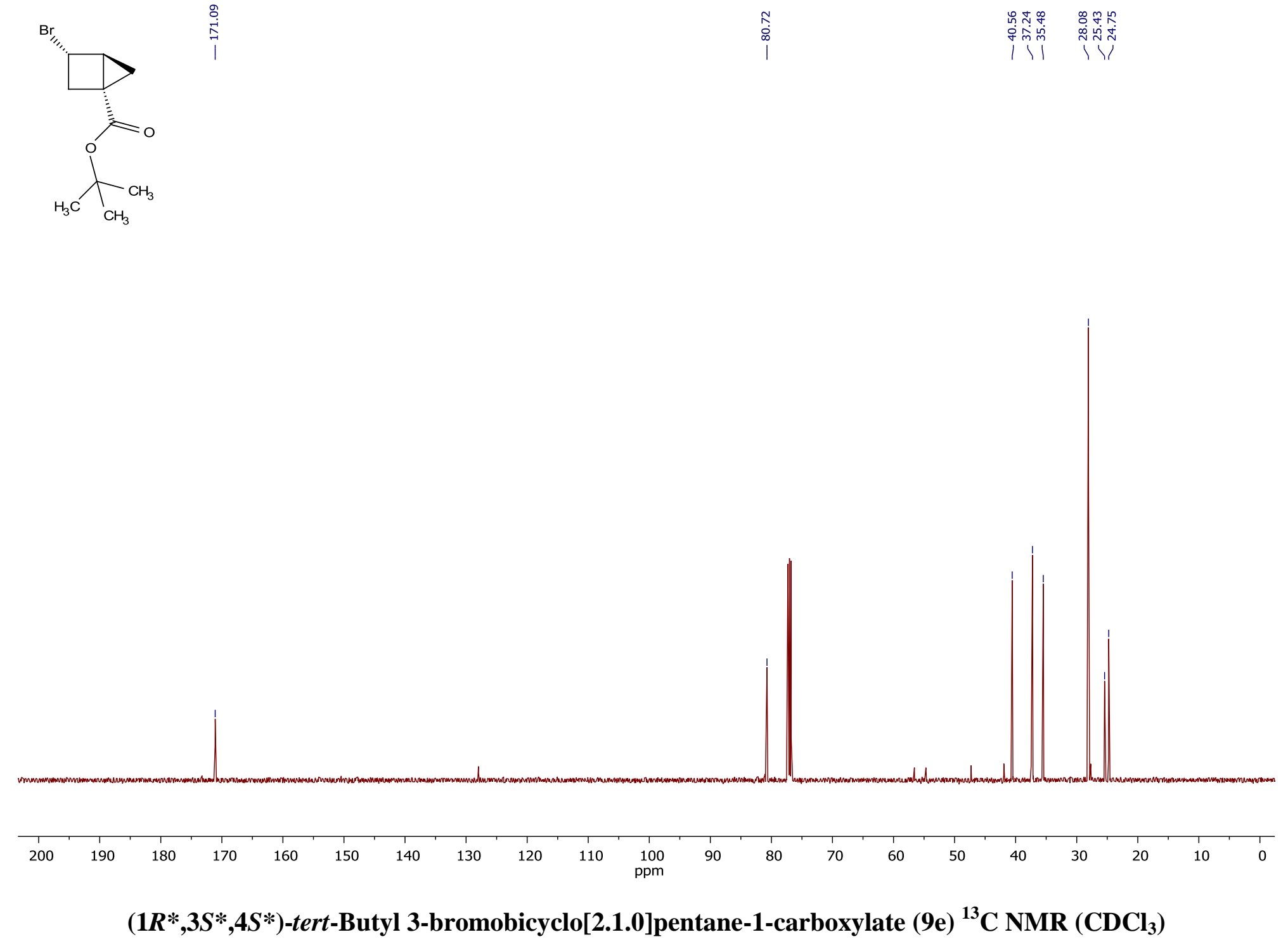


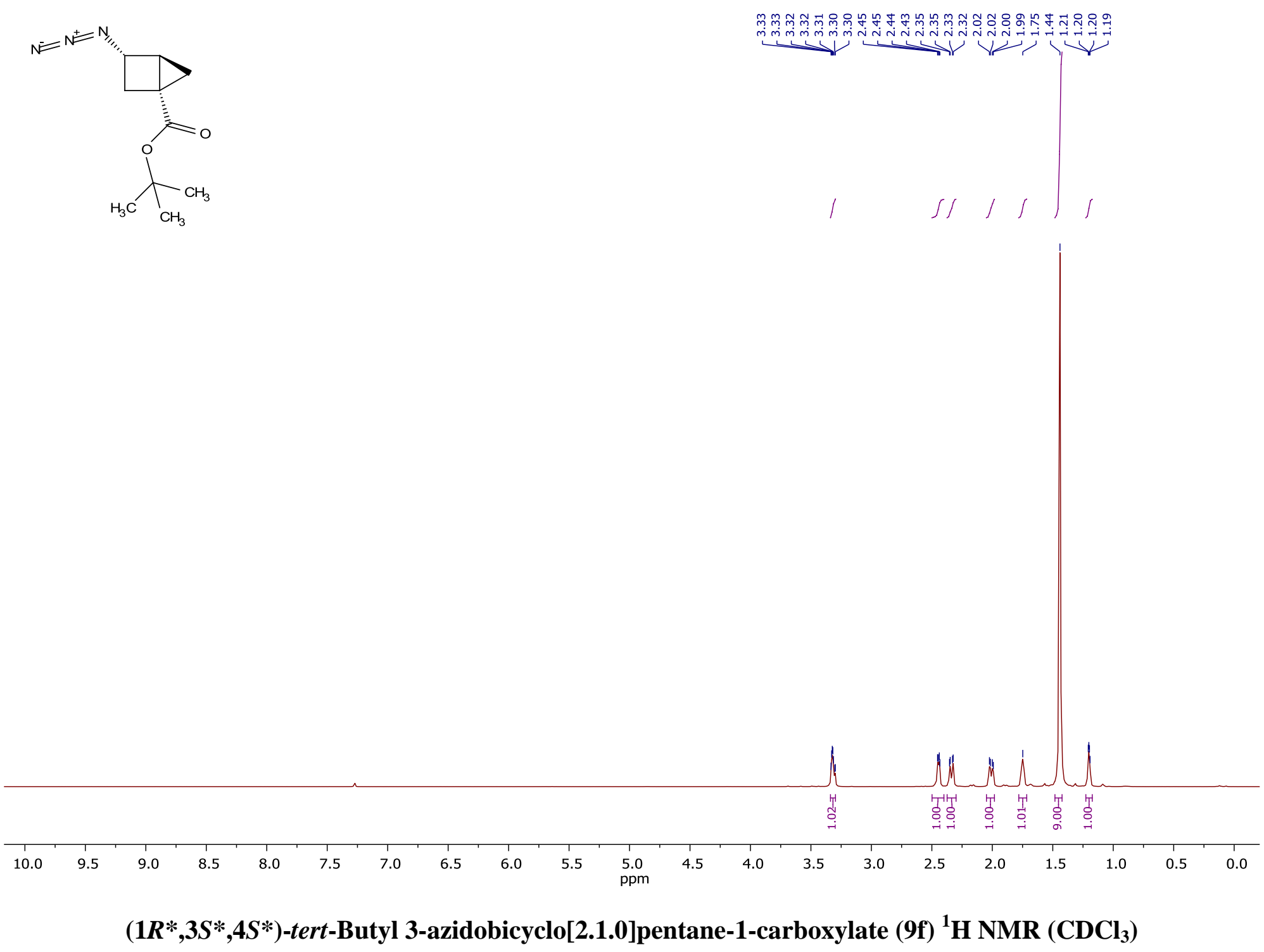



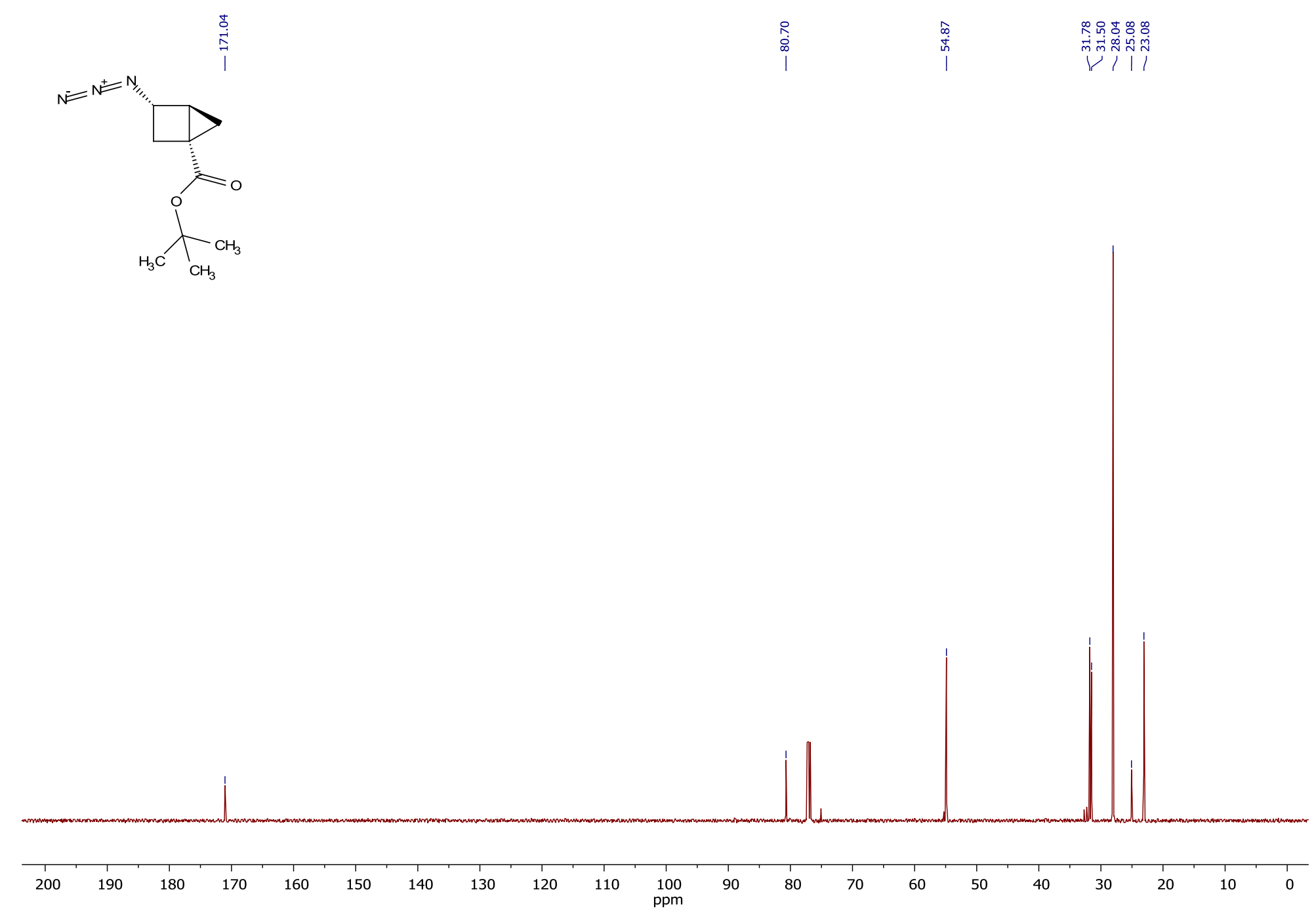

$\left(1 R^{*}, 3 S^{*}, 4 S^{*}\right)$-tert-Butyl 3-azidobicyclo[2.1.0]pentane-1-carboxylate $(9 \mathrm{f}){ }^{13} \mathrm{C} \mathrm{NMR}\left(\mathrm{CDCl}_{3}\right)$ 

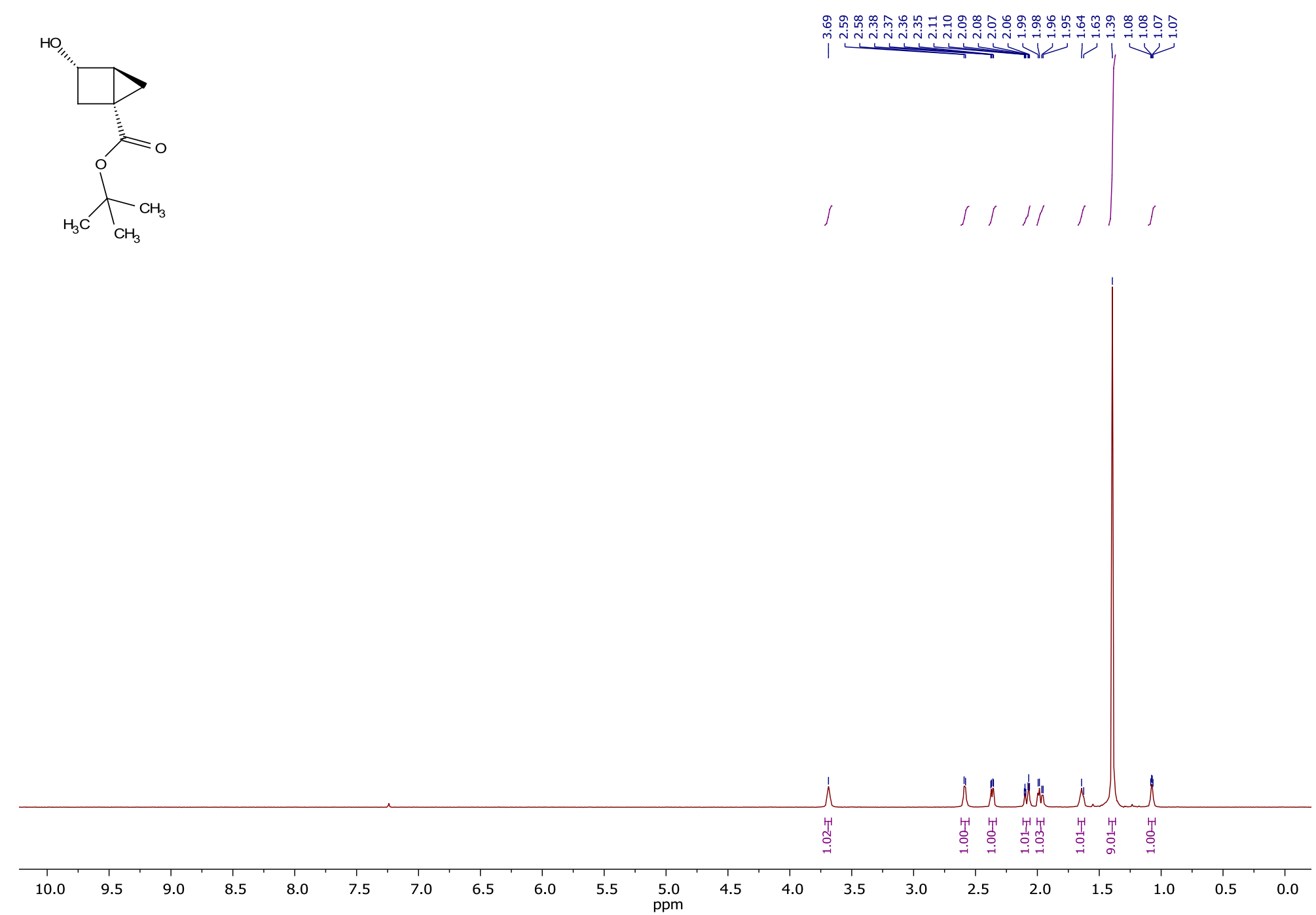

$\left(1 R^{*}, 3 S^{*}, 4 S^{*}\right)$-tert-Butyl 3-hydroxybicyclo[2.1.0]pentane-1-carboxylate (9g) ${ }^{1} \mathrm{H}$ NMR $\left(\mathrm{CDCl}_{3}\right)$ 

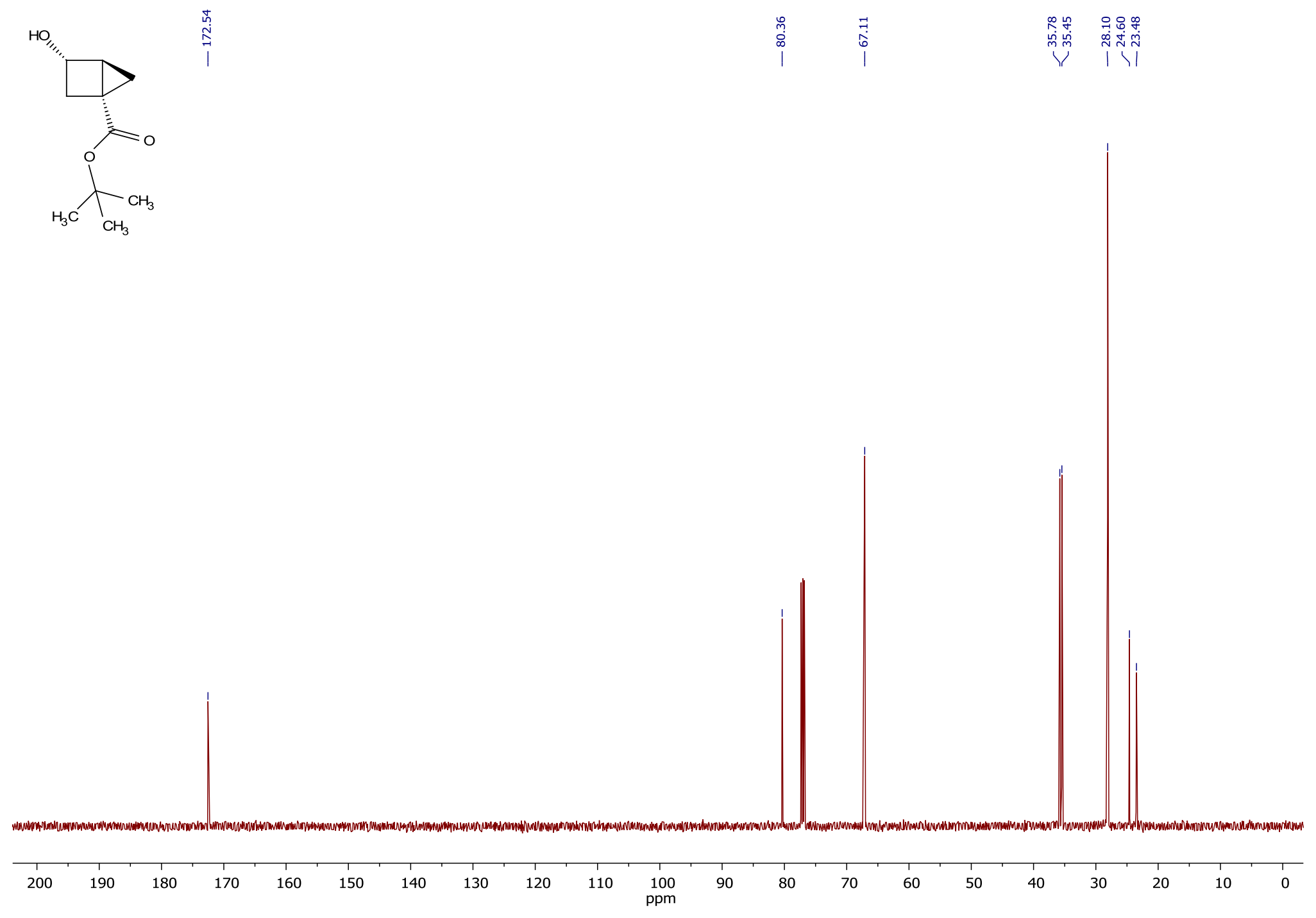

$\left(1 R^{*}, 3 S^{*}, 4 S^{*}\right)$-tert-Butyl 3-hydroxybicyclo[2.1.0]pentane-1-carboxylate $(9 \mathrm{~g}){ }^{13} \mathrm{C} \mathrm{NMR}\left(\mathrm{CDCl}_{3}\right)$ 


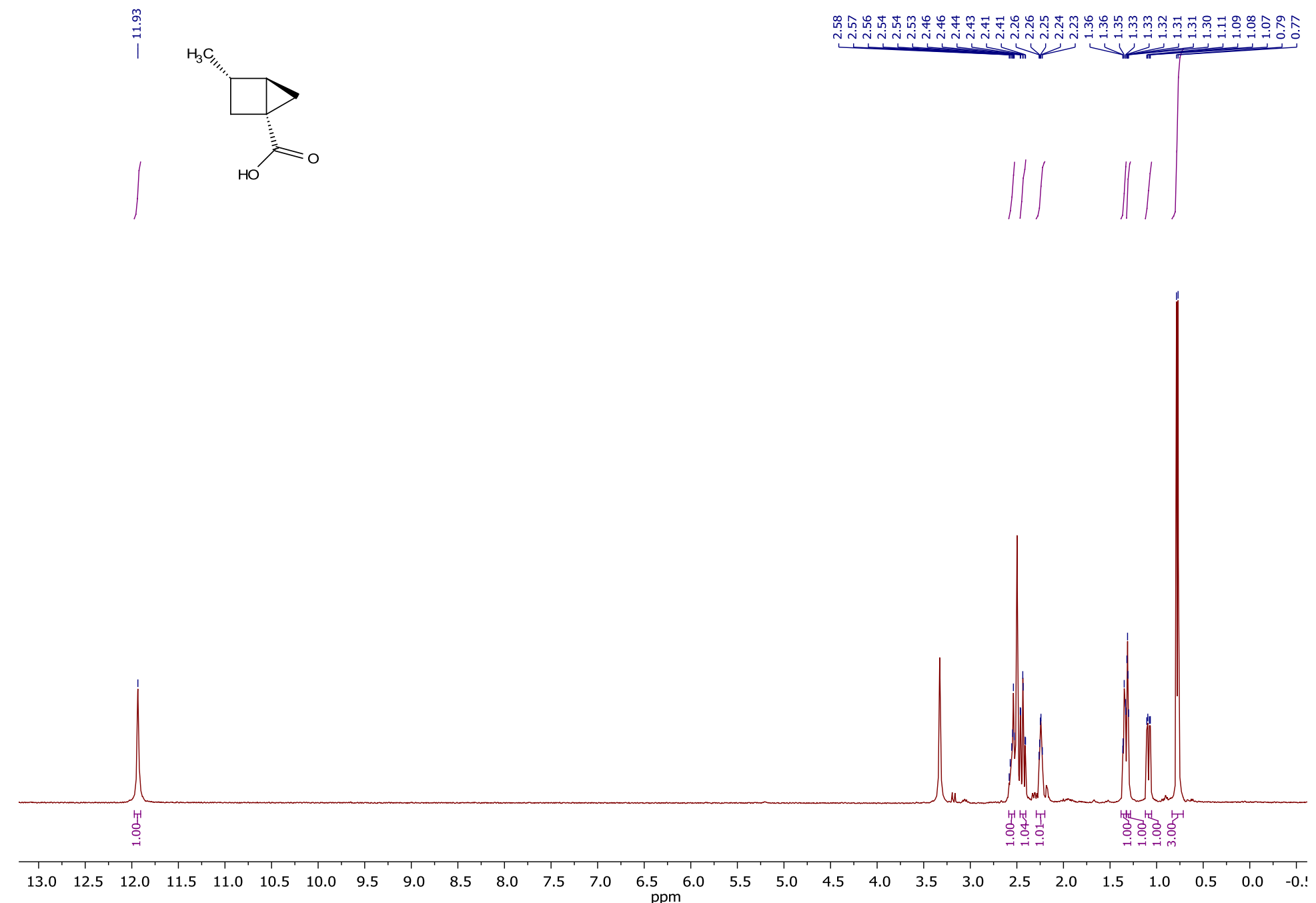

$\left(1 R^{*}, 3 S^{*}, 4 R^{*}\right)-3-M e t h y l b i c y c l o[2.1 .0]$ pentane-1-carboxylic acid (10a) ${ }^{1}$ H NMR (DMSO-d $)$ 


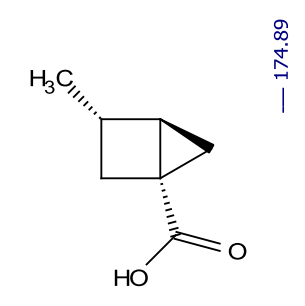

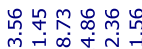

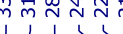

$111 \backslash 1$

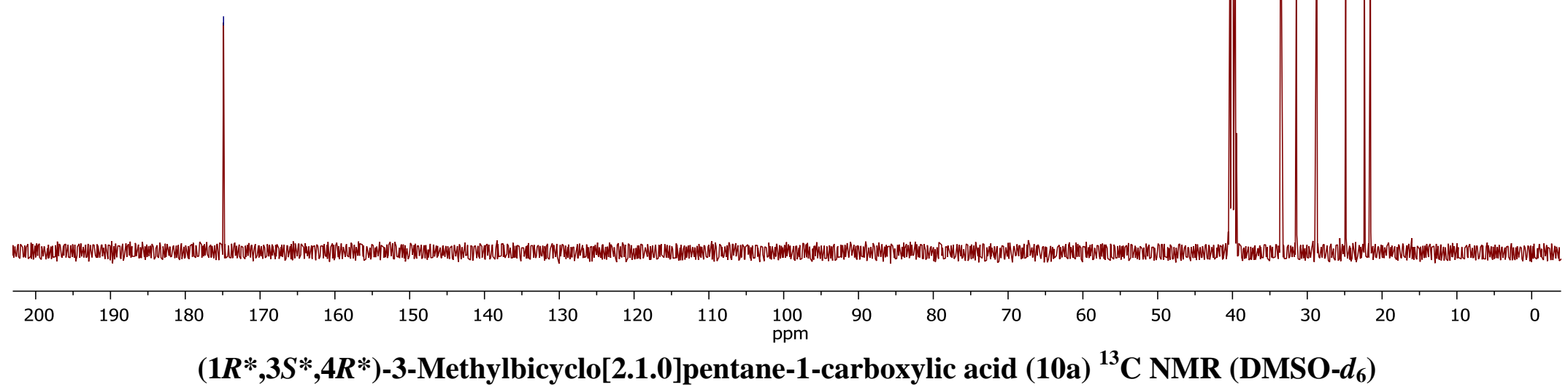



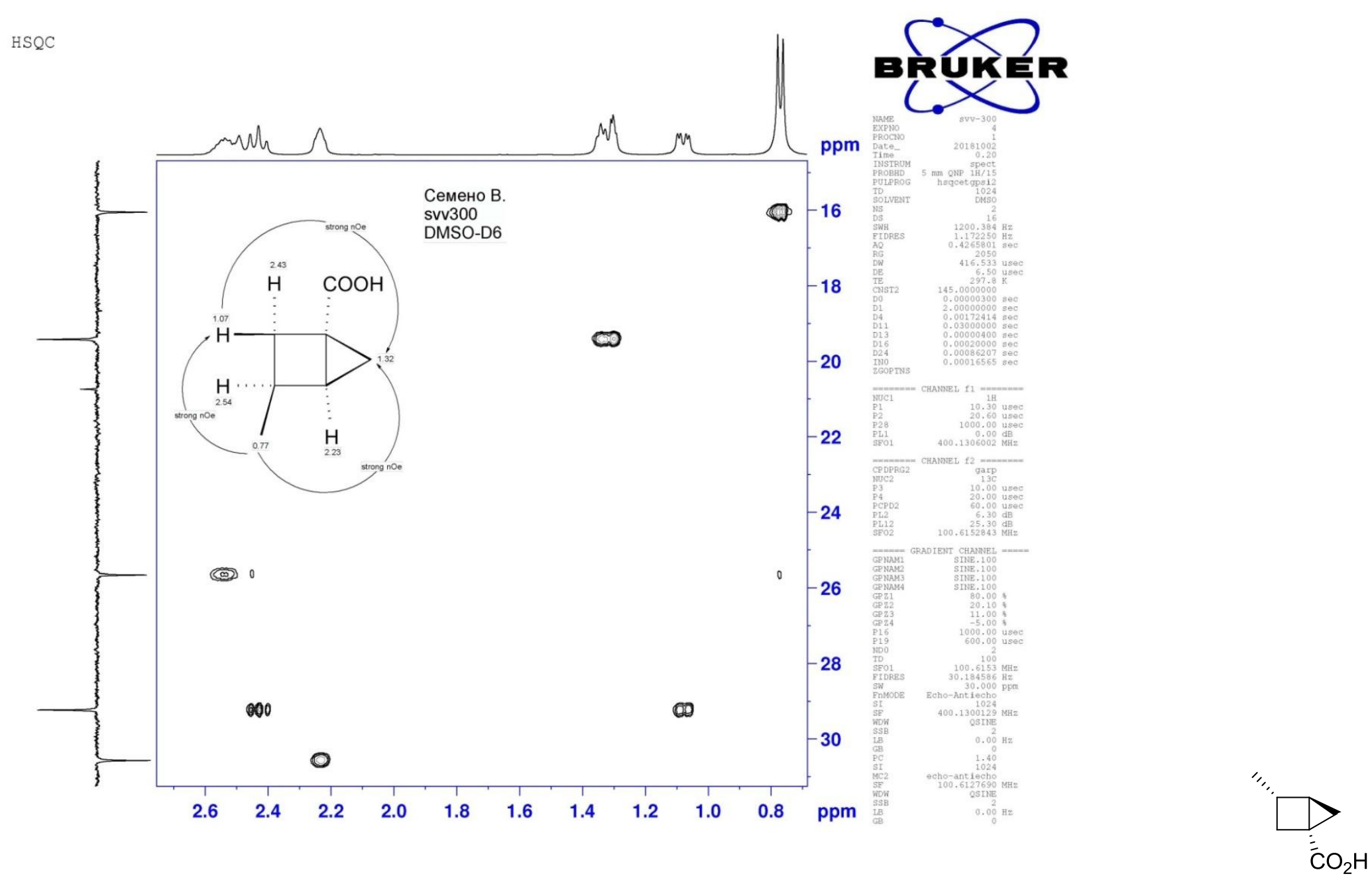

$\left(1 R^{*}, 3 S^{*}, 4 R^{*}\right)-3-$ Methylbicyclo[2.1.0]pentane-1-carboxylic acid (10a) (HSQC) (DMSO- $d_{6}$ ) 


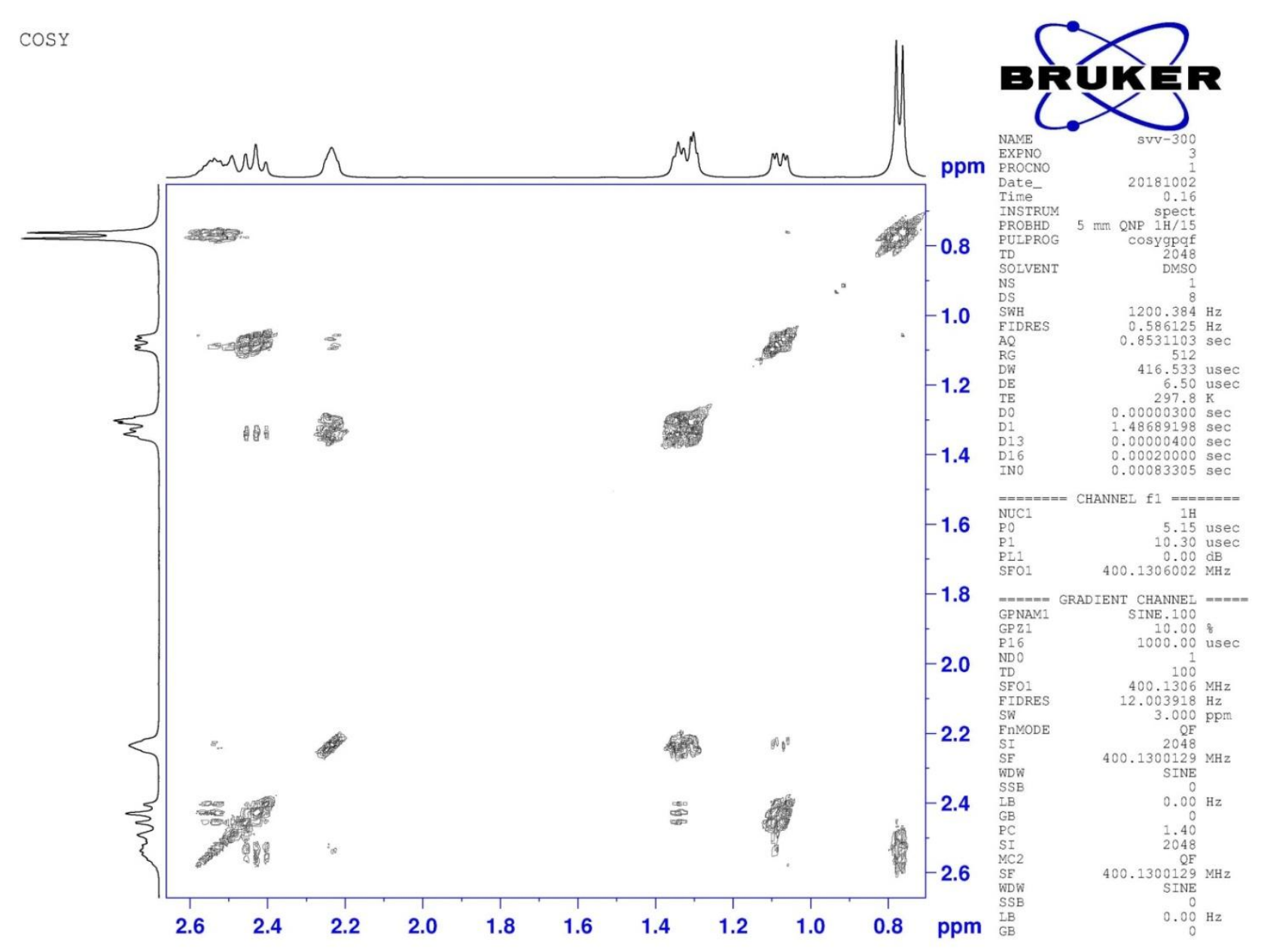




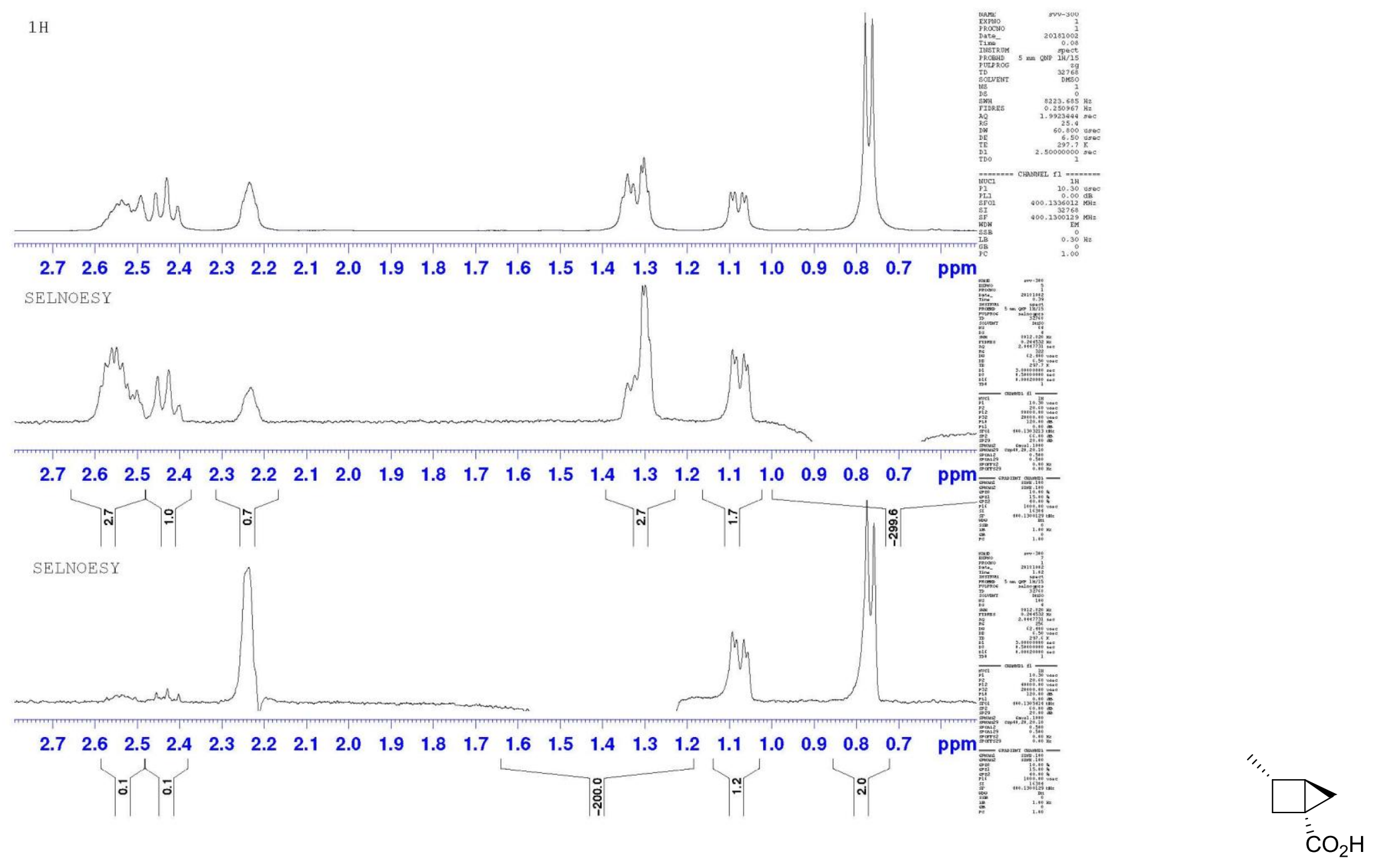

$\left(1 R^{*}, 3 S^{*}, 4 R^{*}\right)-3-M e t h y l b i c y c l o[2.1 .0]$ pentane-1-carboxylic acid (10a) (H-H NOE) (DMSO-d $)$ 

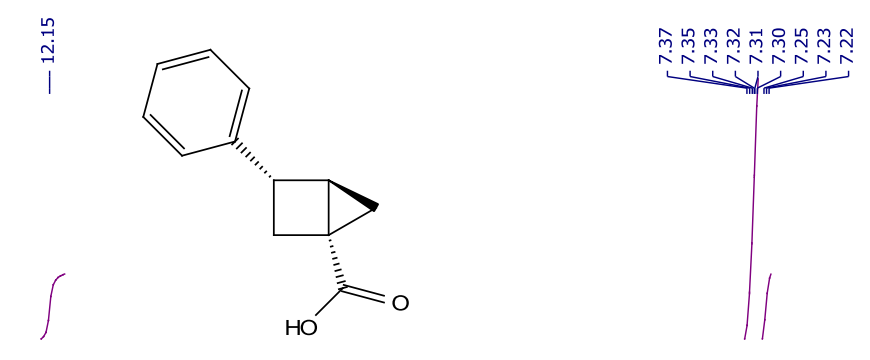

$\underbrace{\infty}$
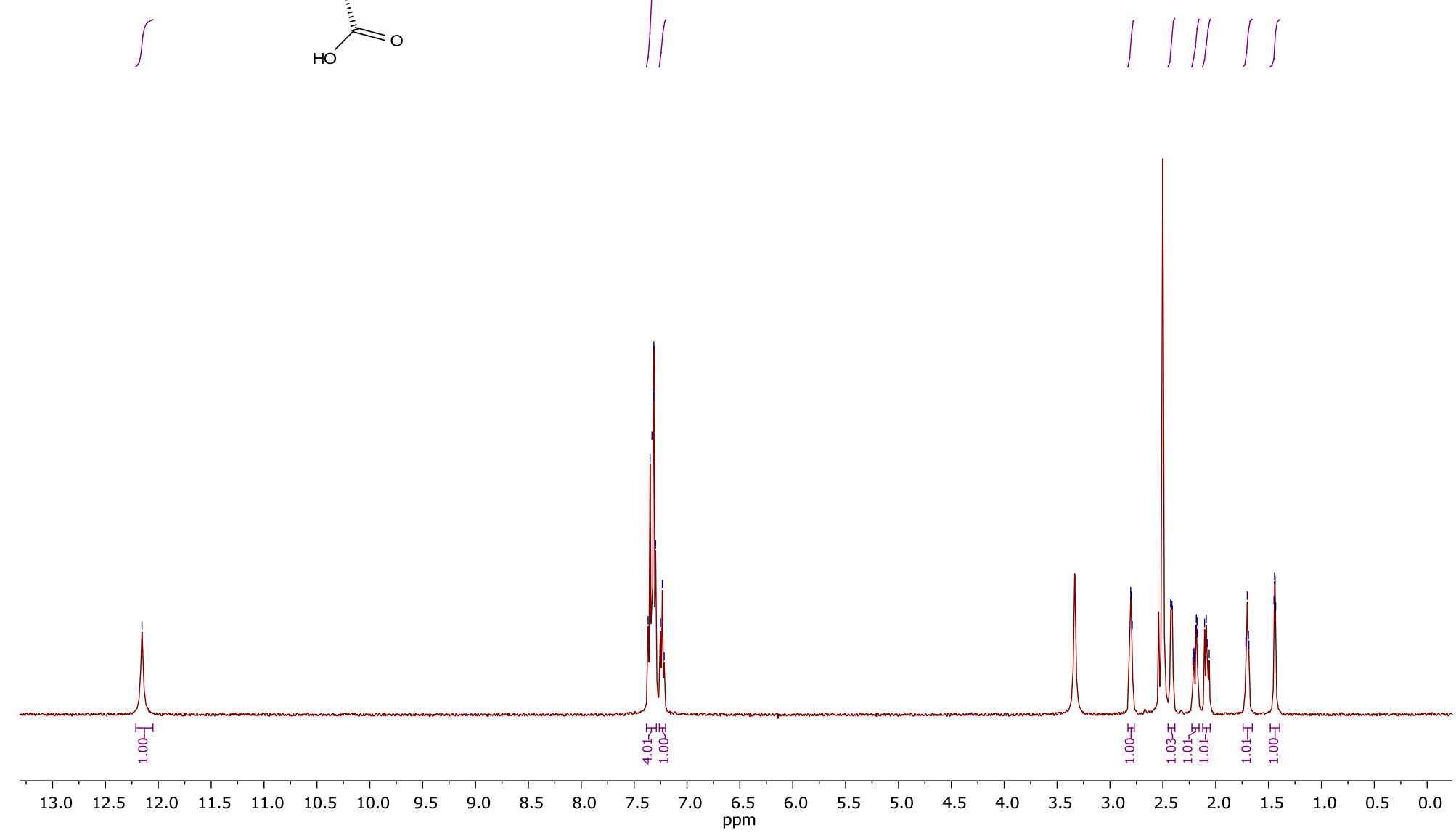

$\left(1 R^{*}, 3 S^{*}, 4 R^{*}\right)$-3-Phenylbicyclo[2.1.0]pentane-1-carboxylic acid (10b) ${ }^{1}$ H NMR (DMSO- $\left.d_{6}\right)$ 


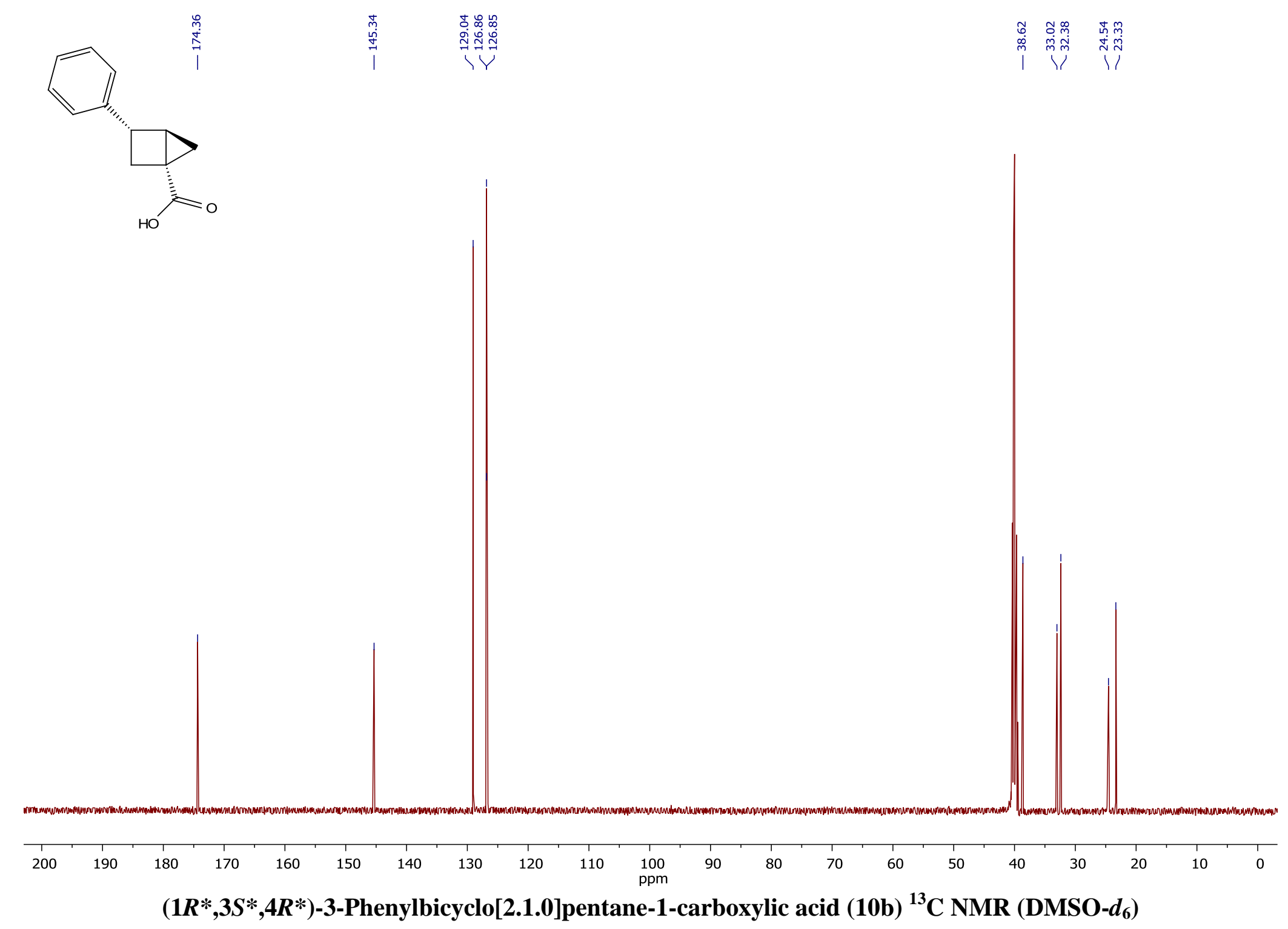



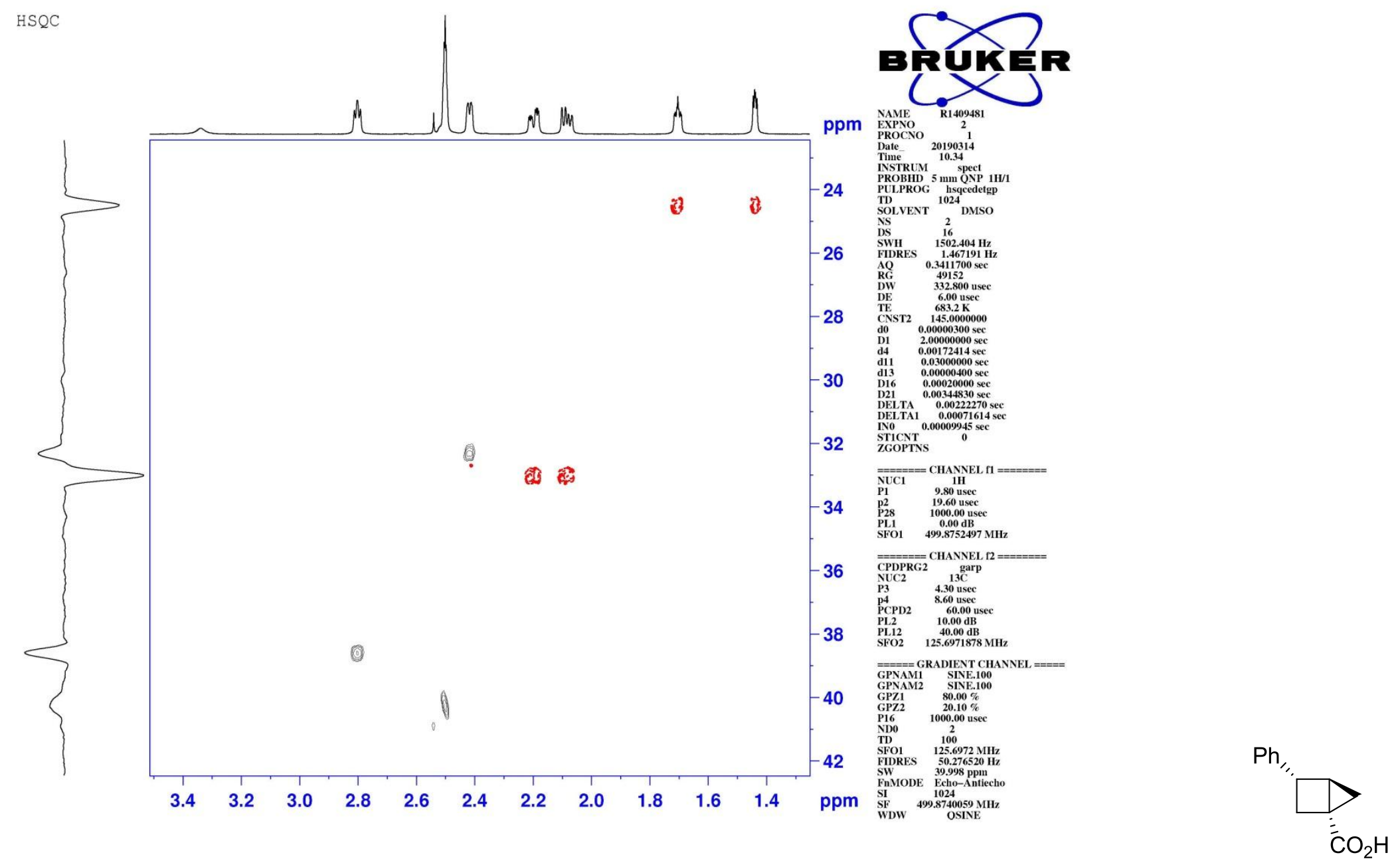

$\left(1 R^{*}, 3 S^{*}, 4 R^{*}\right)-3-$ Phenylbicyclo[2.1.0]pentane-1-carboxylic acid (10b) (HSQC) (DMSO- $\left.d_{6}\right)$ 


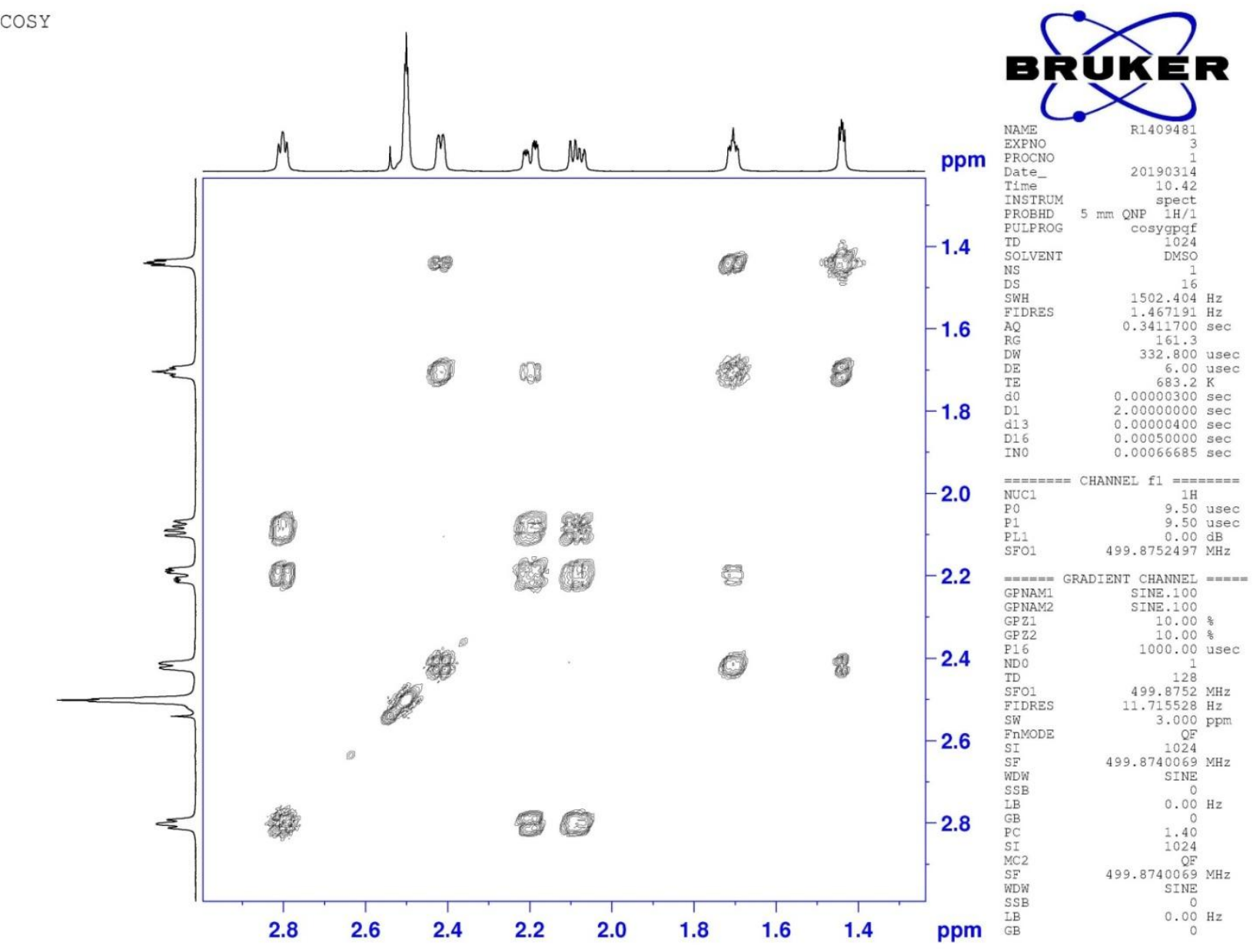

$\mathrm{Ph}$

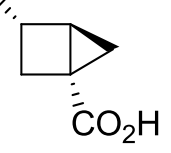

$\left(1 R^{*}, 3 S^{*}, 4 R^{*}\right)-3-P h e n y l b i c y c l o[2.1 .0]$ pentane-1-carboxylic acid (10b) (COSY) (DMSO- $\left.d_{6}\right)$ 

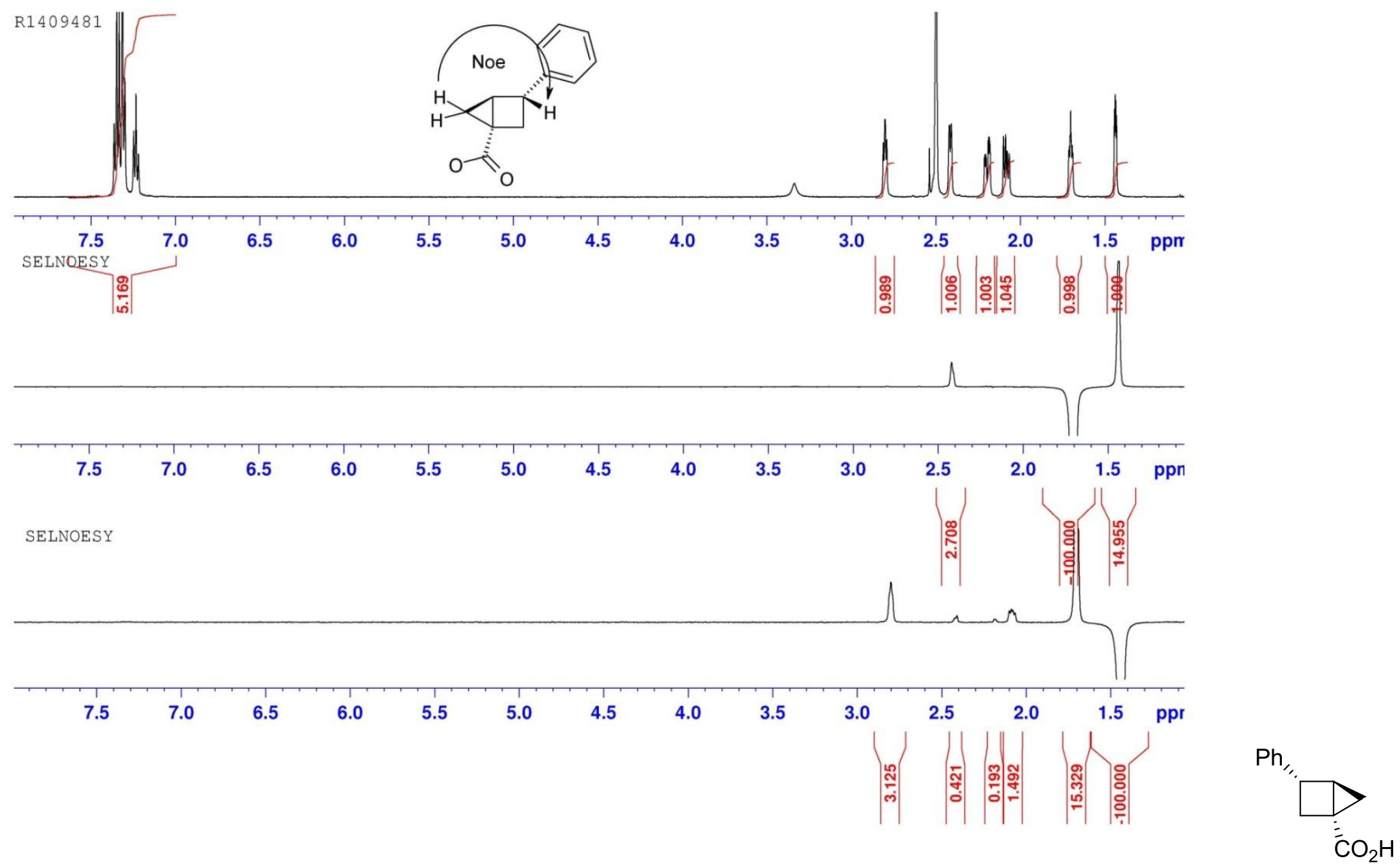

$\left(1 R^{*}, 3 S^{*}, 4 R^{*}\right)-3-P h e n y l b i c y c l o[2.1 .0]$ pentane-1-carboxylic acid (10b) (H-H NOE) (DMSO-d $)$ 

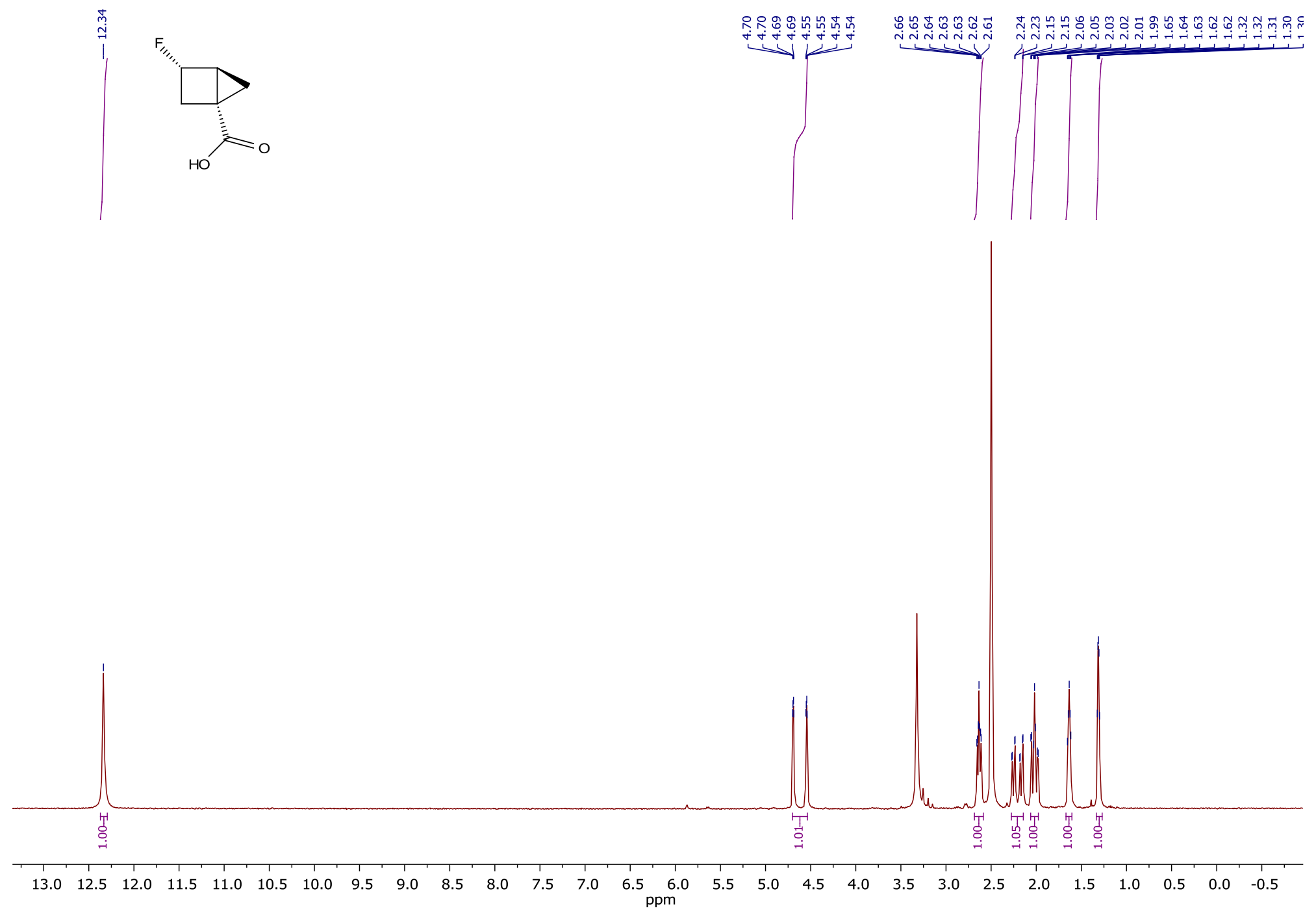

$\left(1 R^{*}, 3 S^{*}, 4 S^{*}\right)$-3-Fluorobicyclo[2.1.0]pentane-1-carboxylic acid (10c) ${ }^{1}$ H NMR (DMSO-d $)$ 


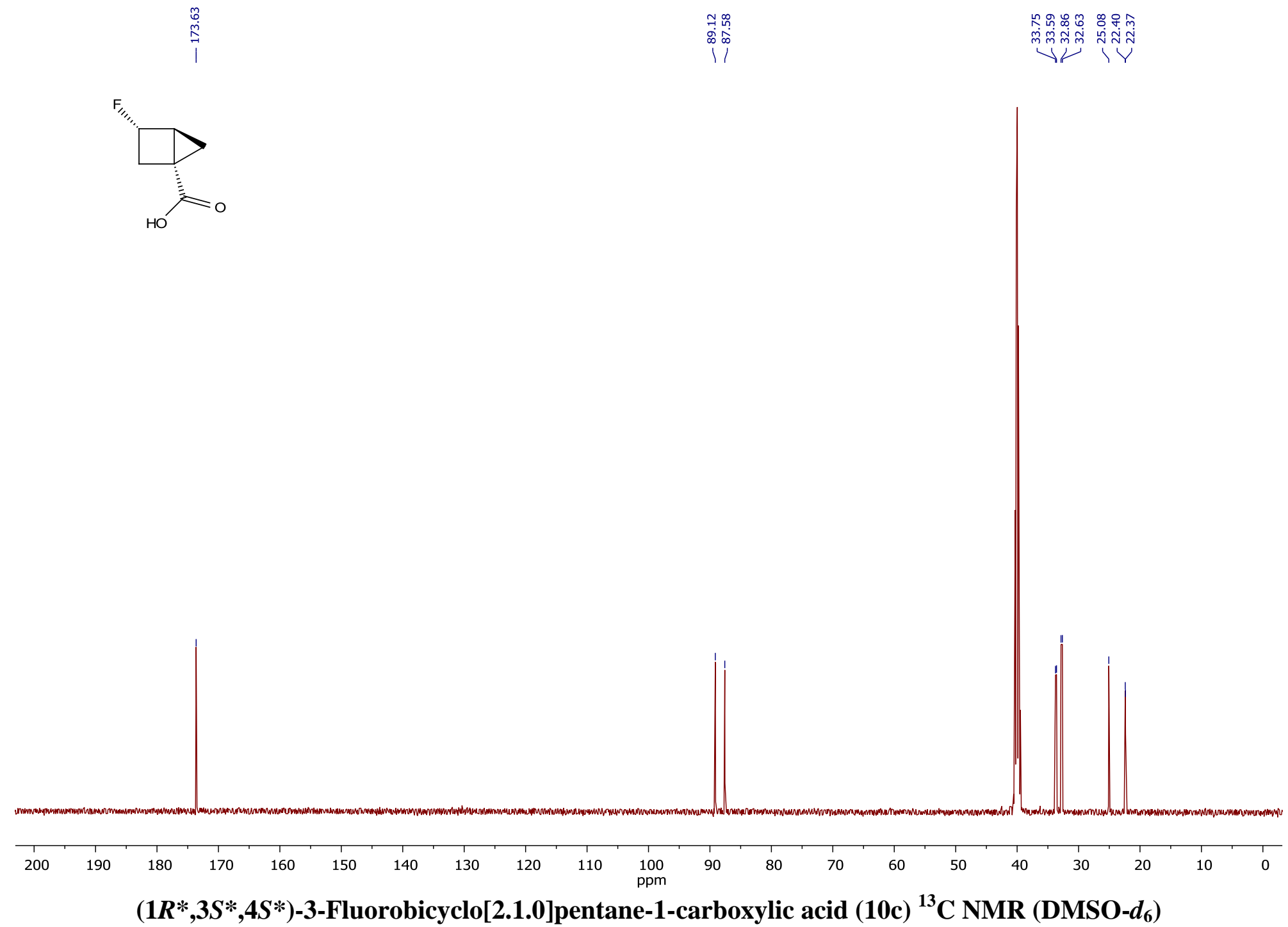




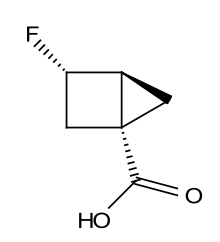

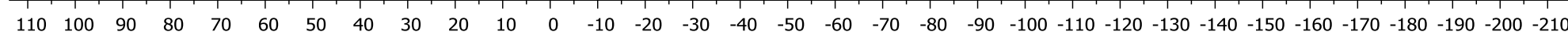

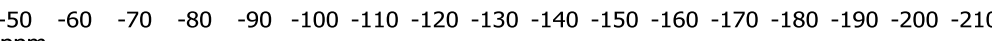

$\left(1 R^{*}, 3 S^{*}, 4 S^{*}\right)$-3-Fluorobicyclo[2.1.0]pentane-1-carboxylic acid (10c) ${ }^{19} \mathrm{~F}$ NMR (DMSO- $d_{6}$ ) 

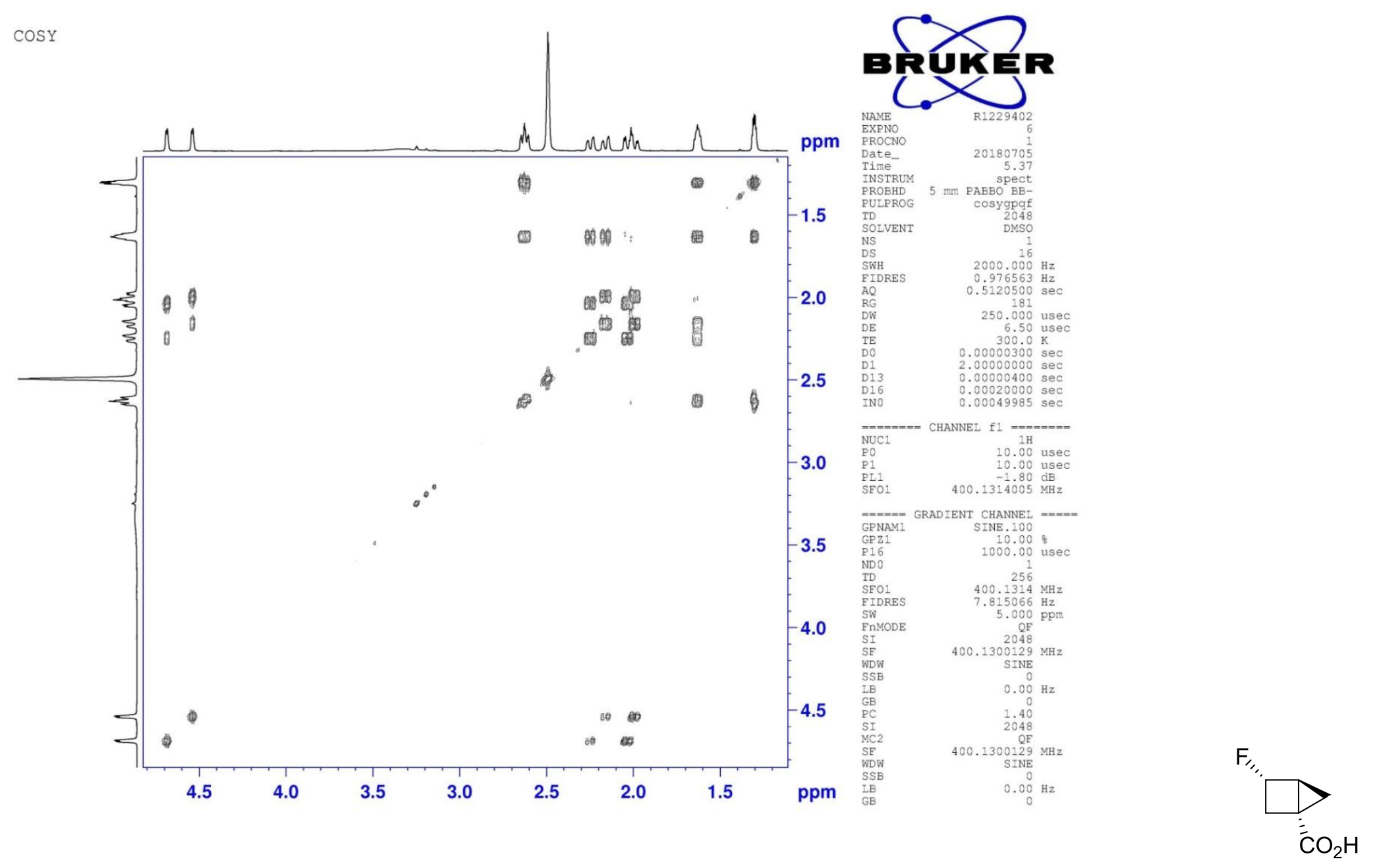

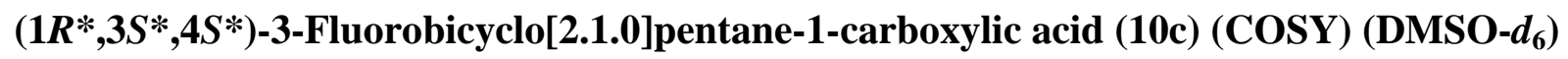



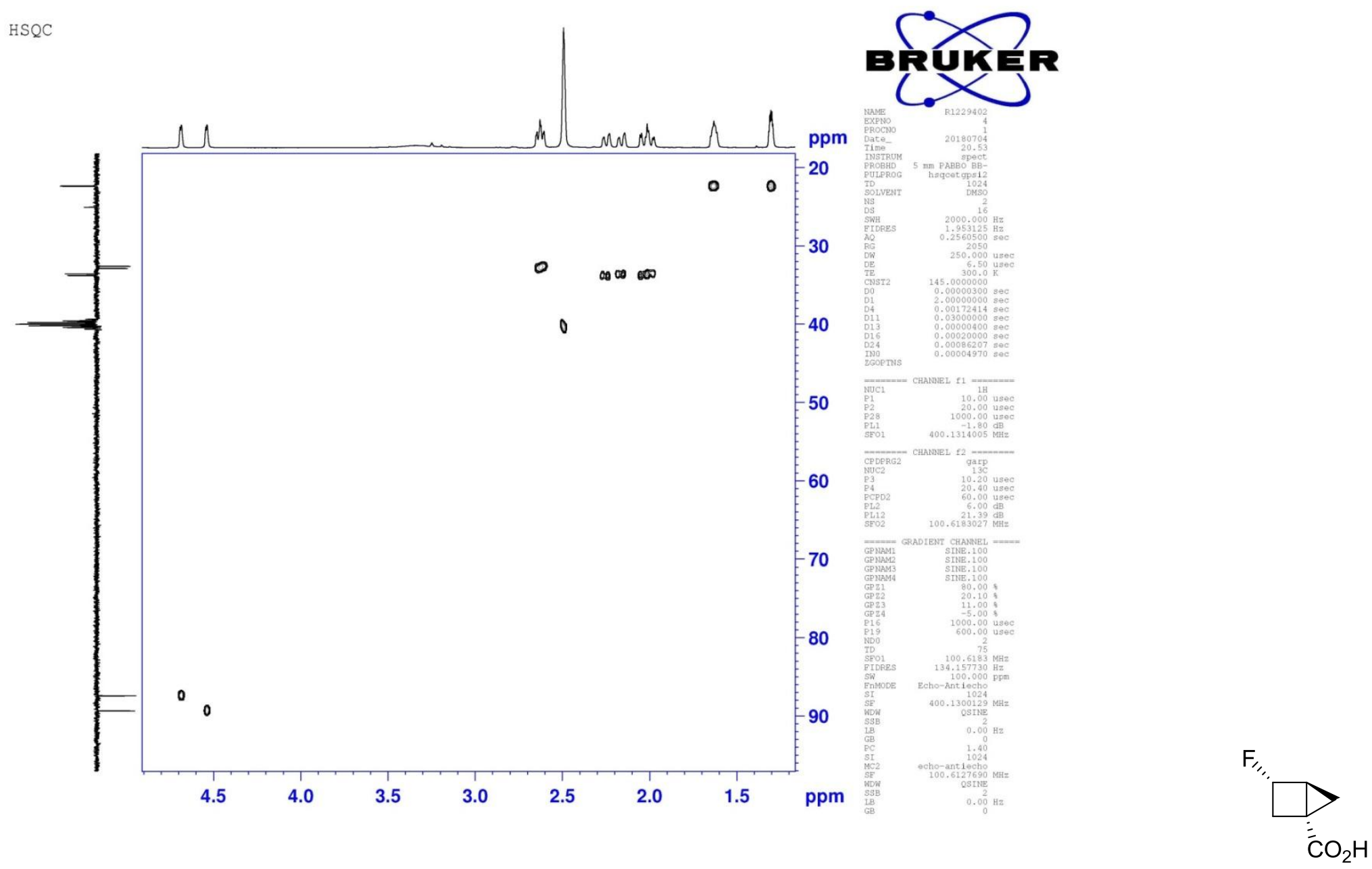

$\left(1 R^{*}, 3 S^{*}, 4 S^{*}\right)-3-$ Fluorobicyclo[2.1.0]pentane-1-carboxylic acid (10c) (HSQC) (DMSO-d $\left.{ }_{6}\right)$ 

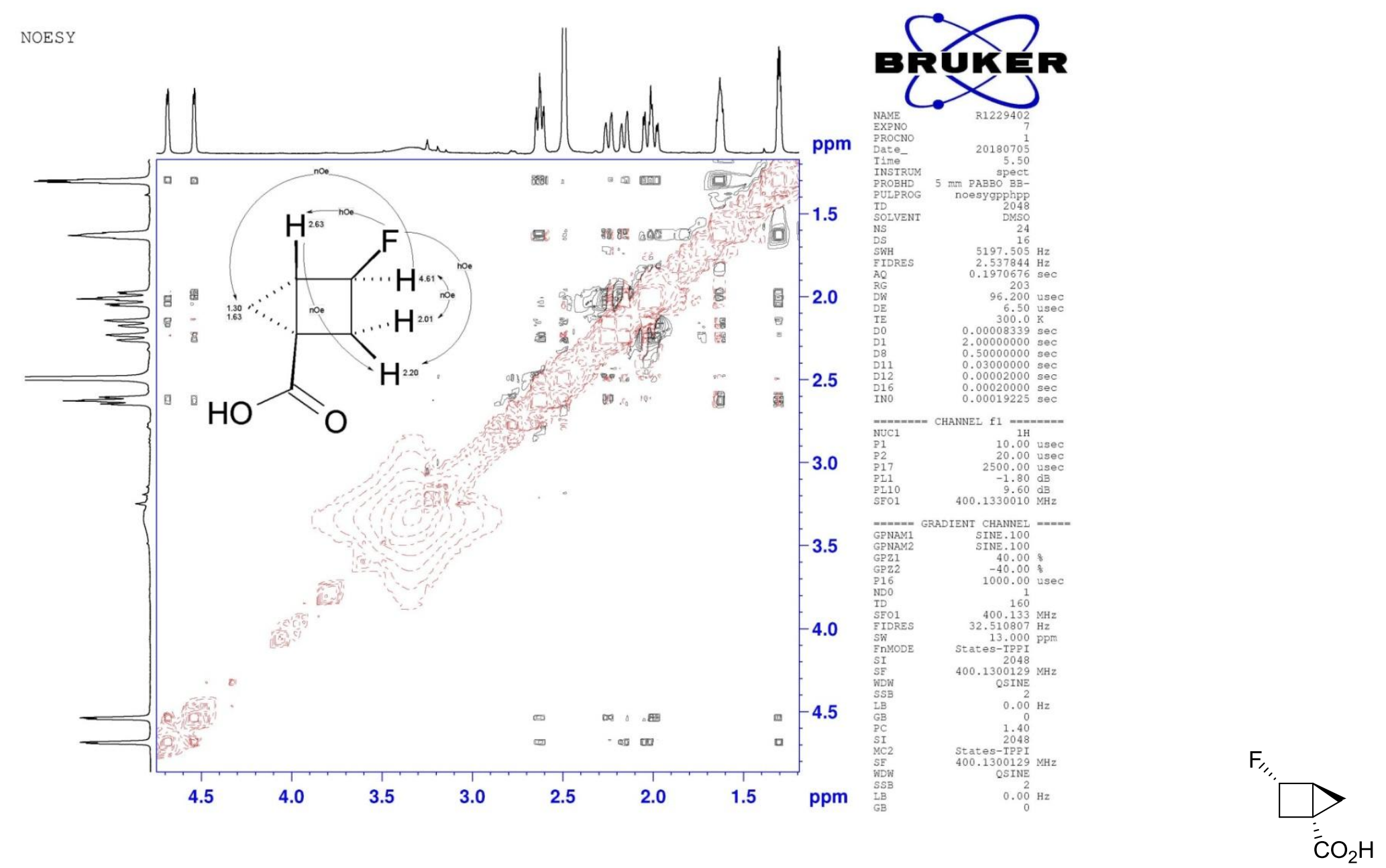

$\left(1 R^{*}, 3 S^{*}, 4 S^{*}\right)$-3-Fluorobicyclo[2.1.0]pentane-1-carboxylic acid (10c) (NOESY) (DMSO-d (D) $^{-}$ 

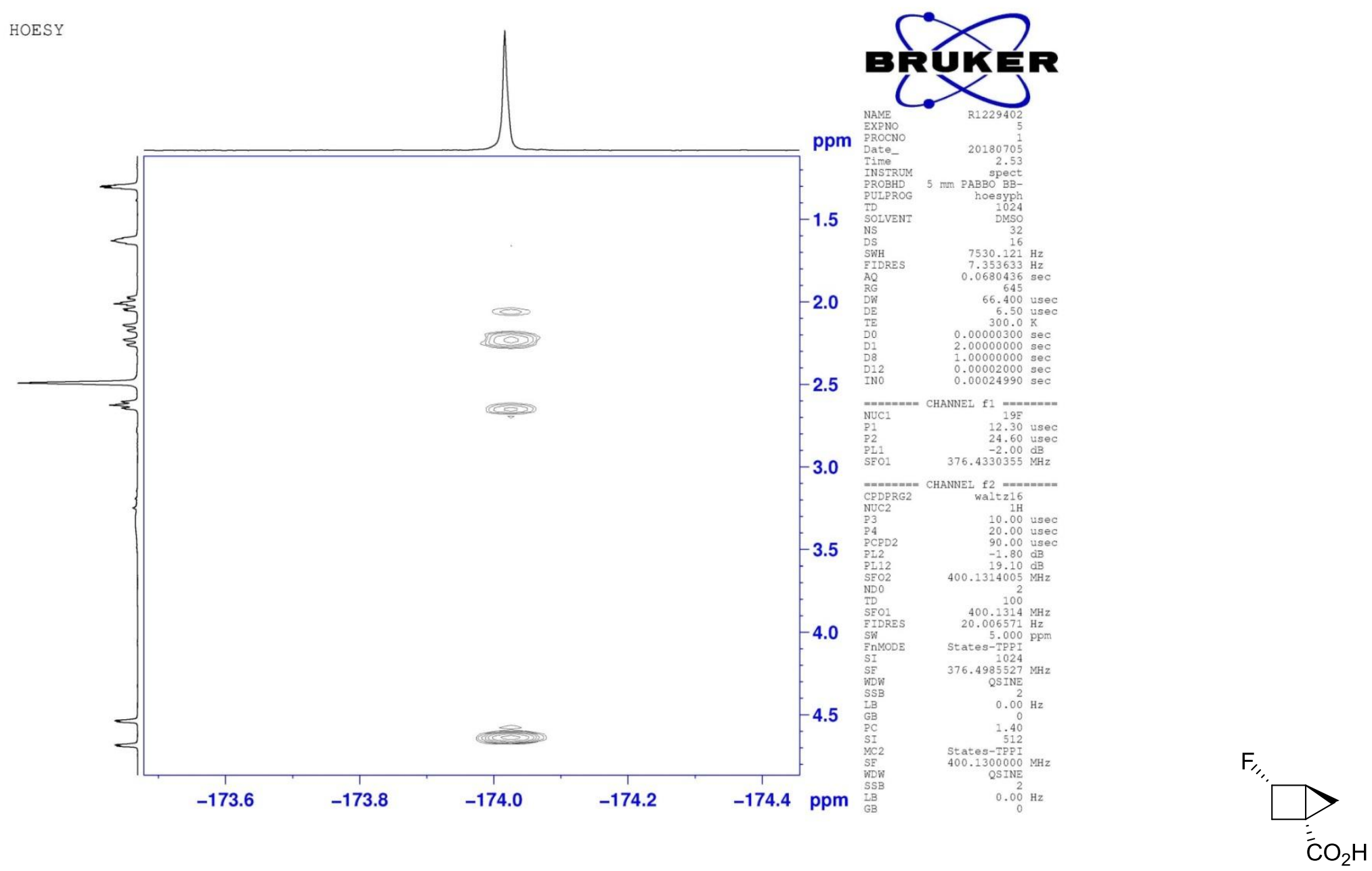

$\left(1 R^{*}, 3 S^{*}, 4 S^{*}\right)$-3-Fluorobicyclo[2.1.0]pentane-1-carboxylic acid (10c) (HOESY) (DMSO-d $\left.)_{6}\right)$ 


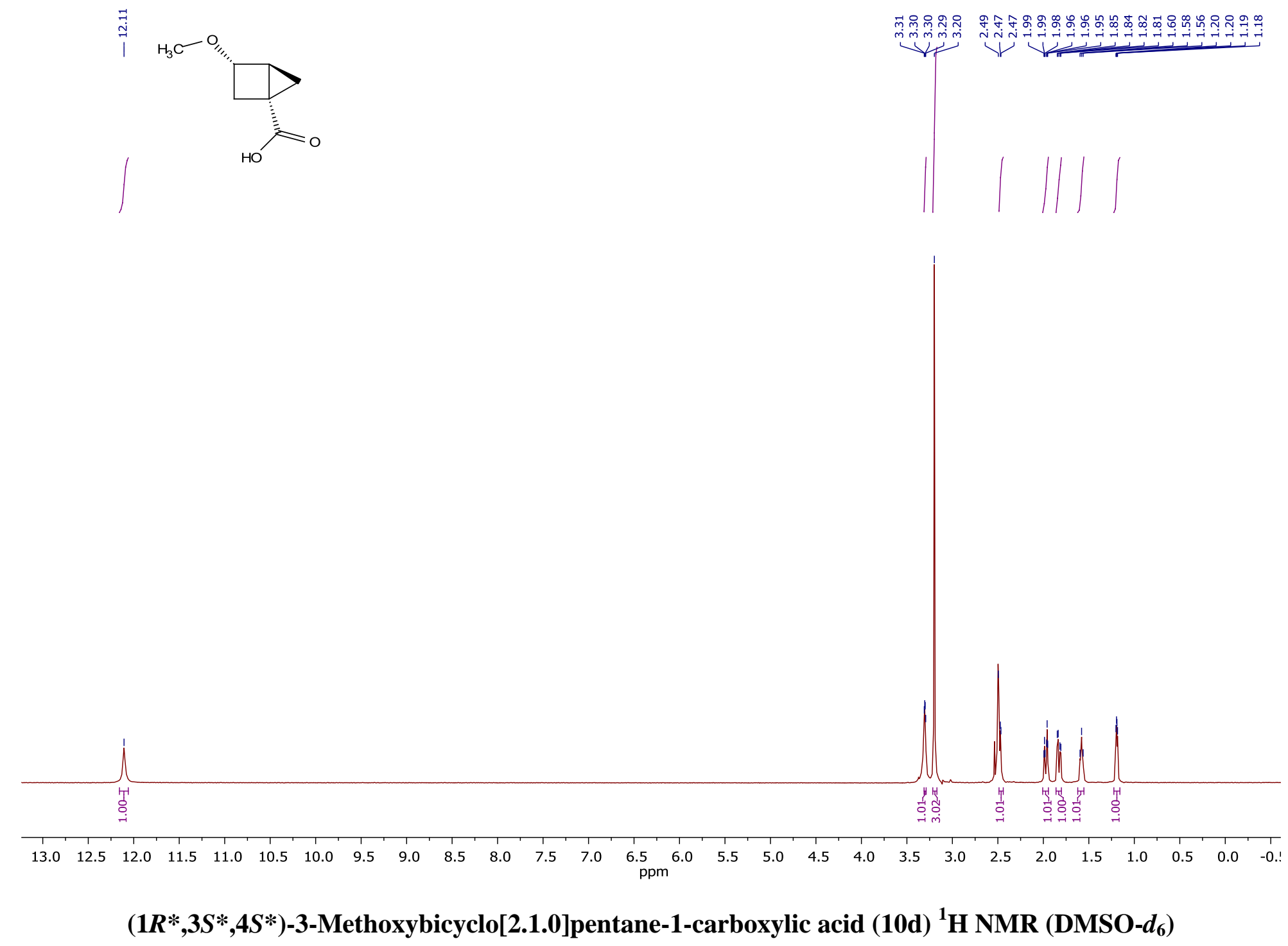



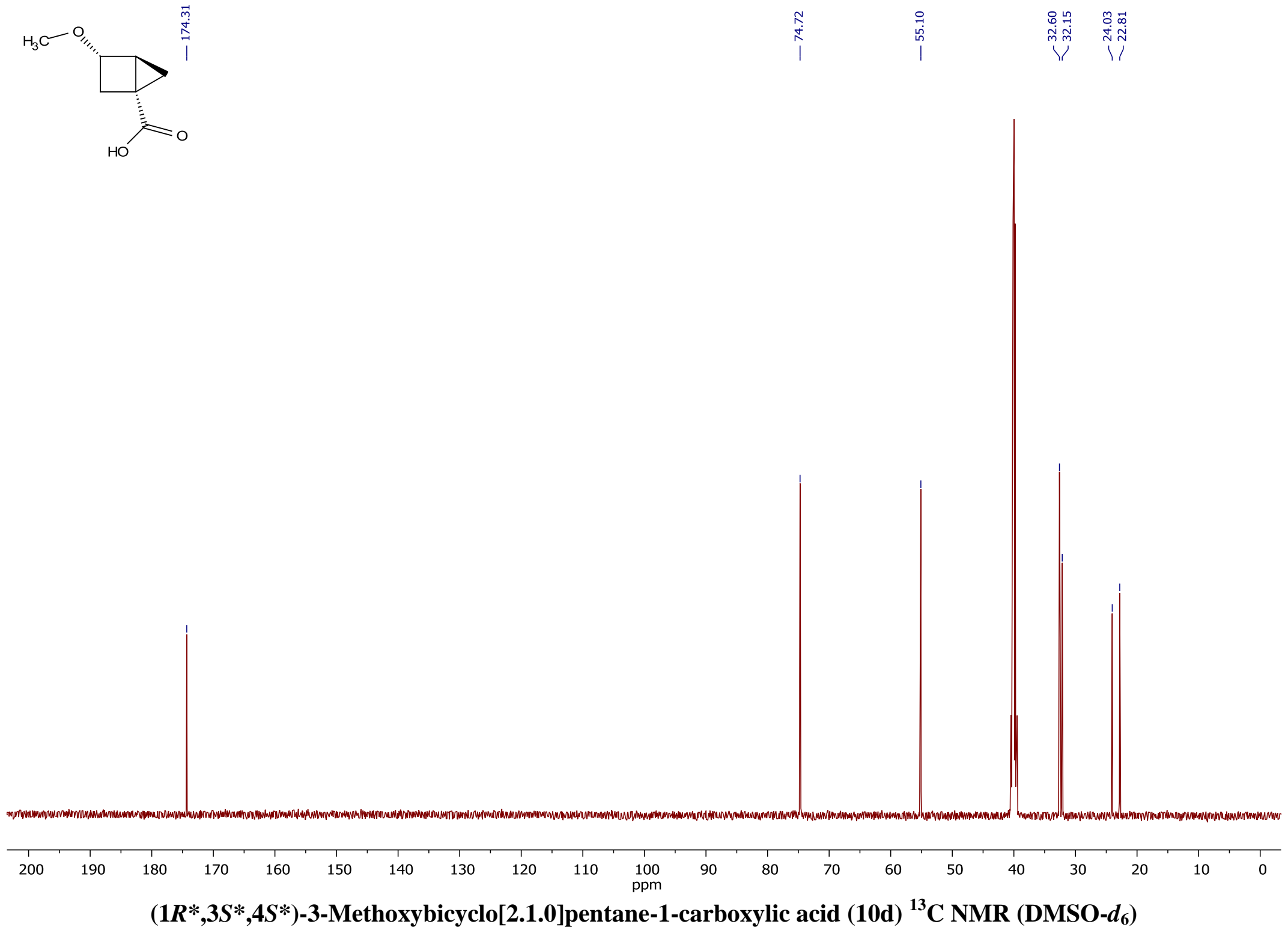

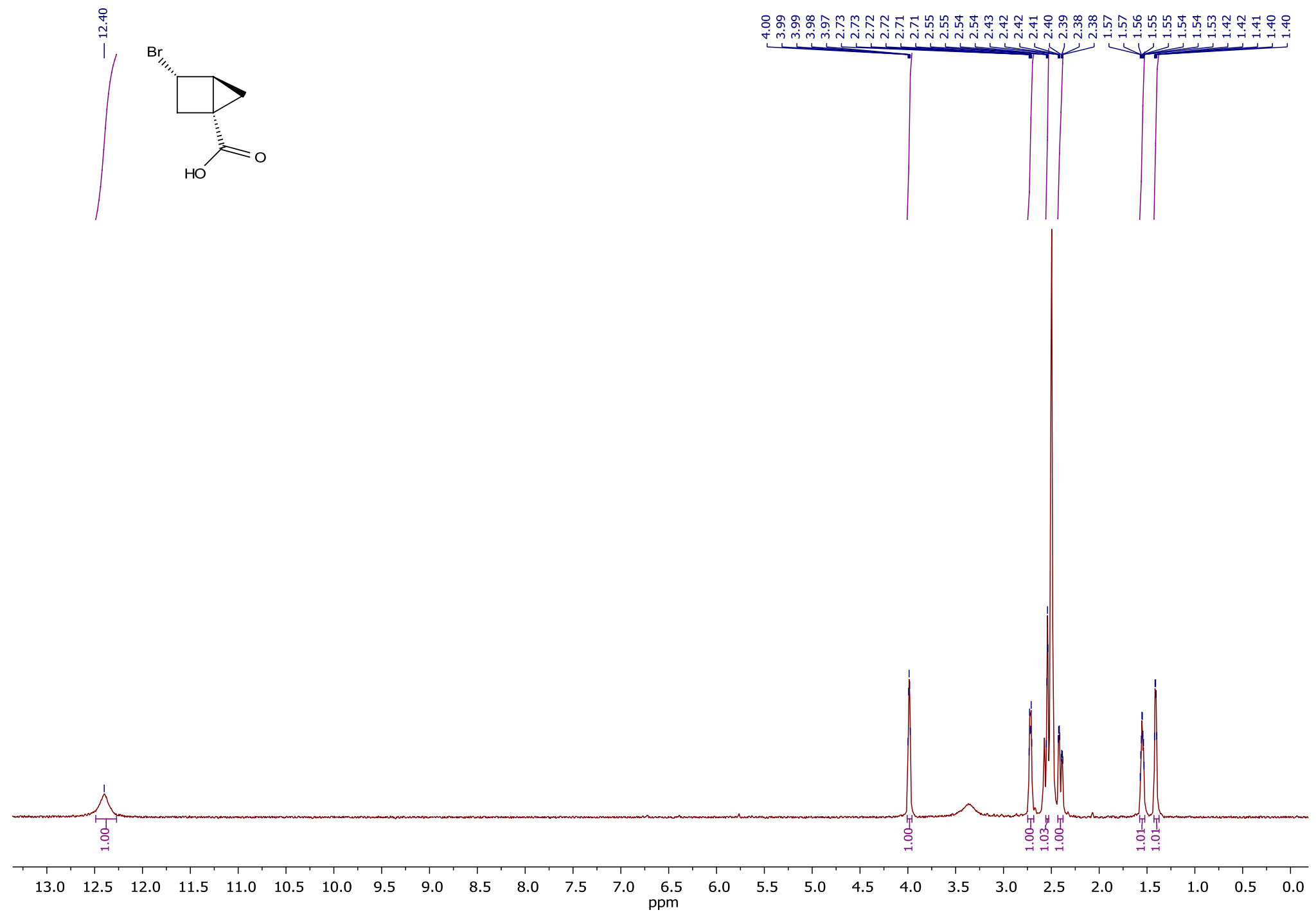

$\left(1 R^{*}, 3 S^{*}, 4 S^{*}\right)-3-B r o m o b i c y c l o[2.1 .0]$ pentane-1-carboxylic acid (10e) ${ }^{1} \mathrm{H}$ NMR (DMSO- $\left.d_{6}\right)$ 

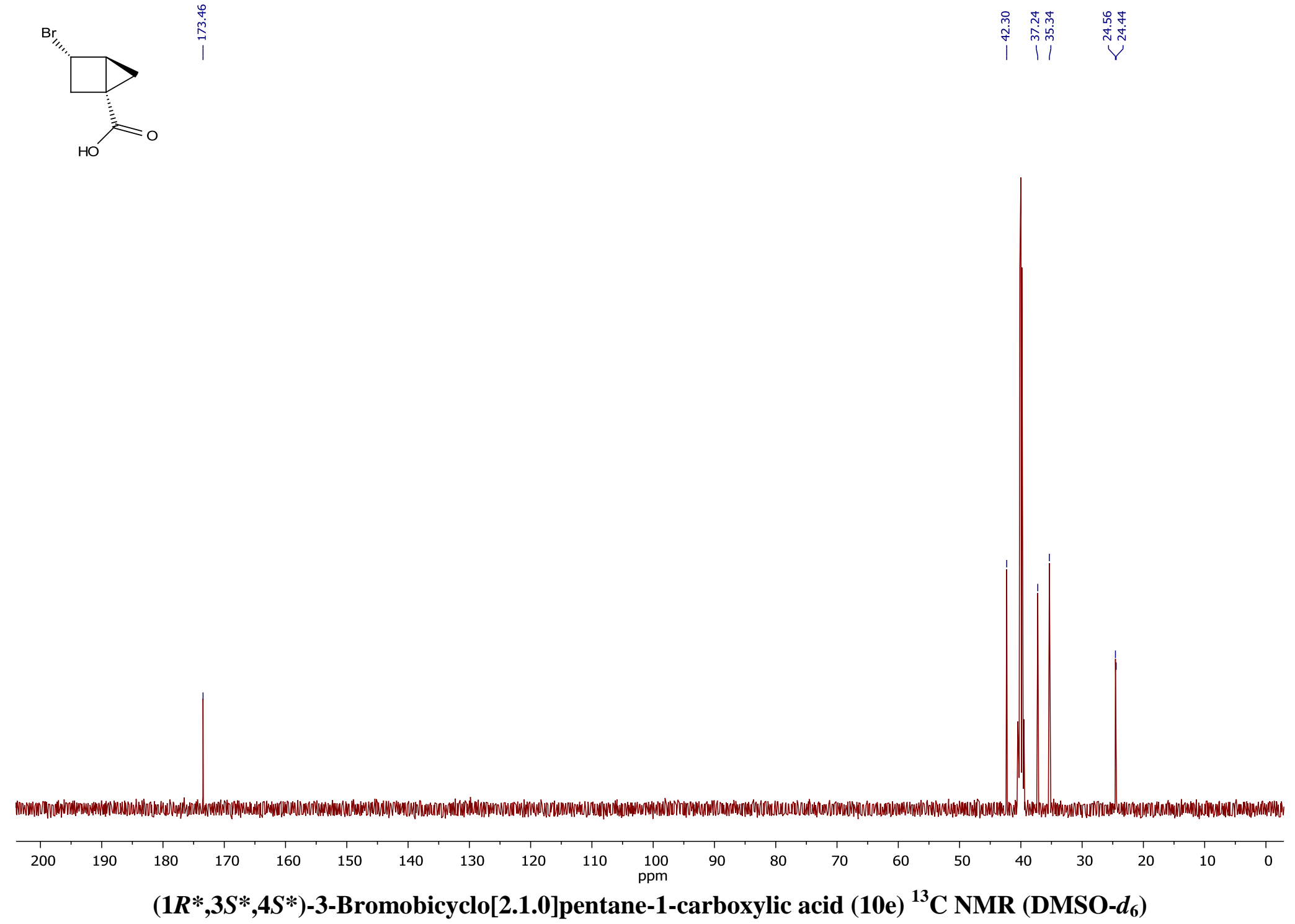


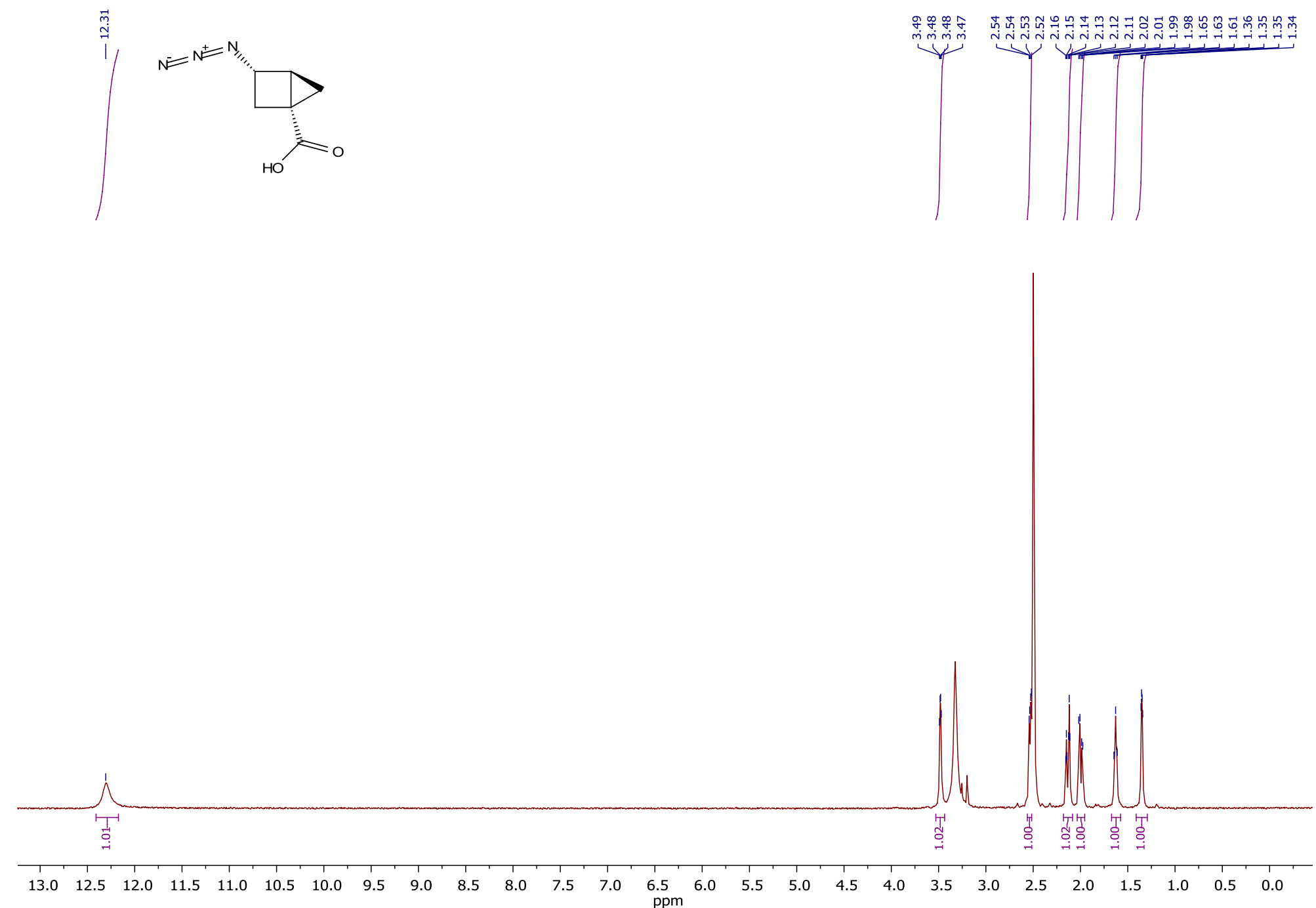

$\left(1 R^{*}, 3 S^{*}, 4 S^{*}\right)$-3-Azidobicyclo[2.1.0]pentane-1-carboxylic acid (10f) ${ }^{1}$ H NMR (DMSO-d $\left.d_{6}\right)$ 

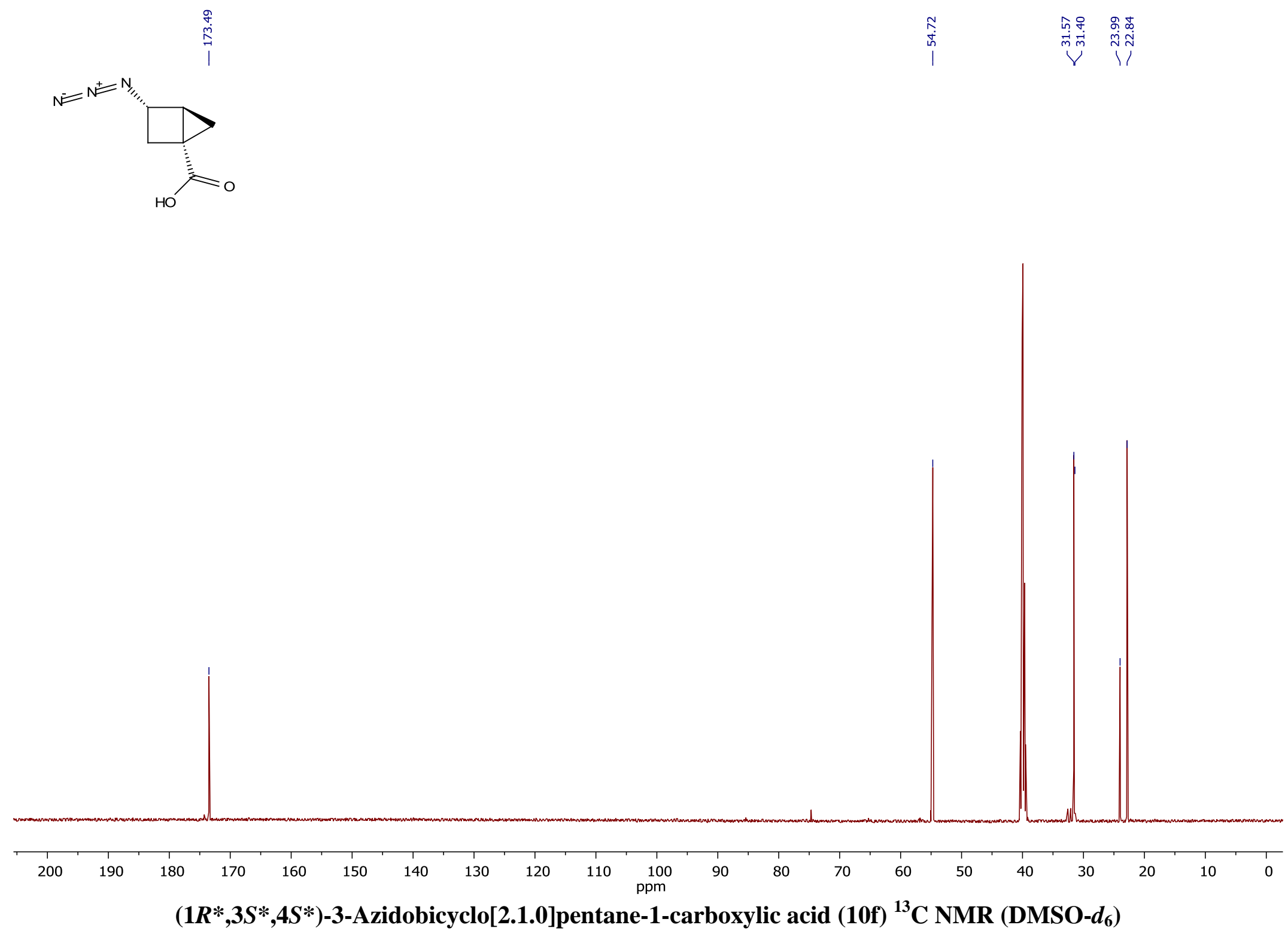

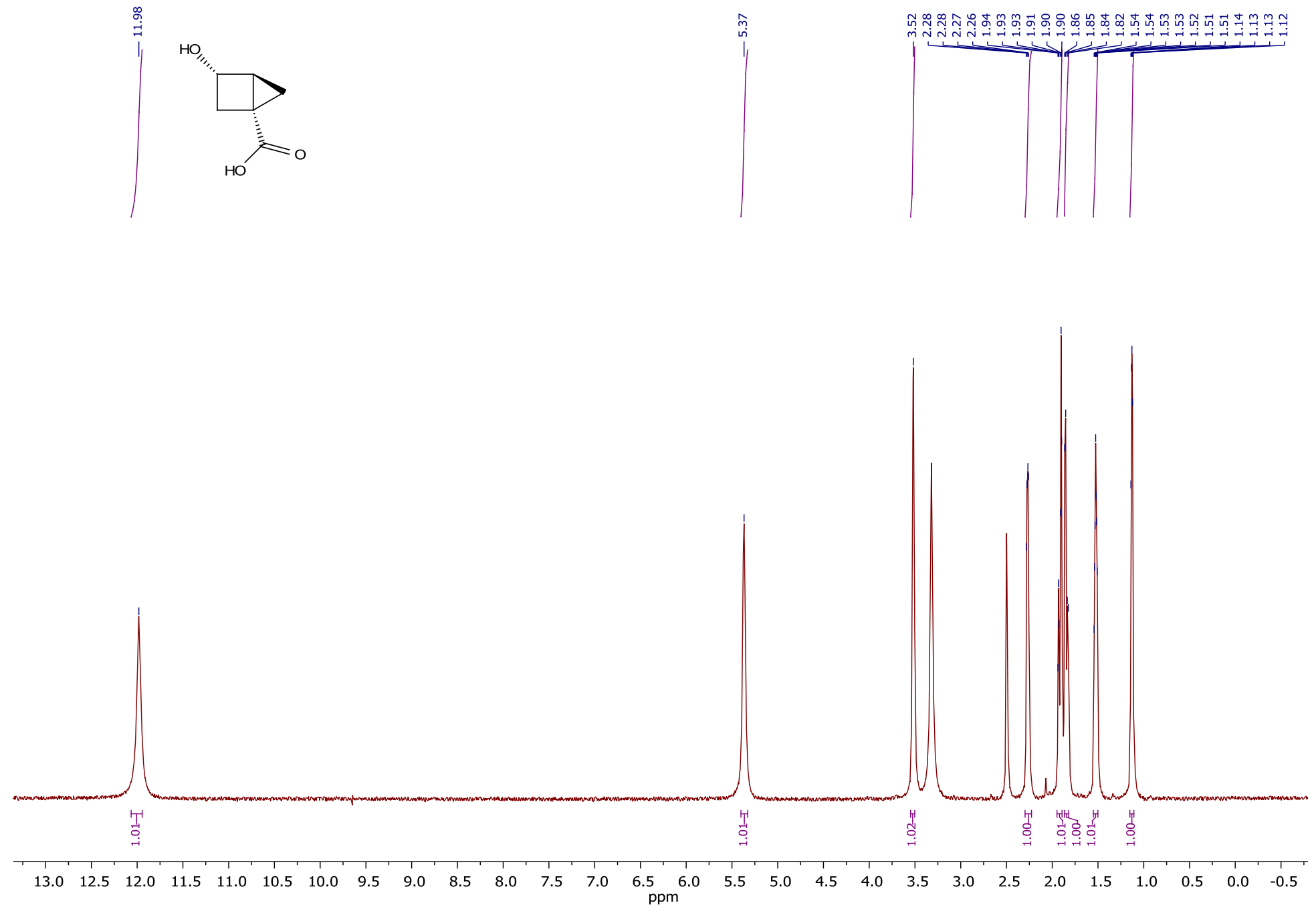

$\left(1 R^{*}, 3 S^{*}, 4 S^{*}\right)-3-H y d r o x y b i c y c l o[2.1 .0]$ pentane-1-carboxylic acid (10g) ${ }^{1}$ H NMR (DMSO-d $\left.)_{6}\right)$ 

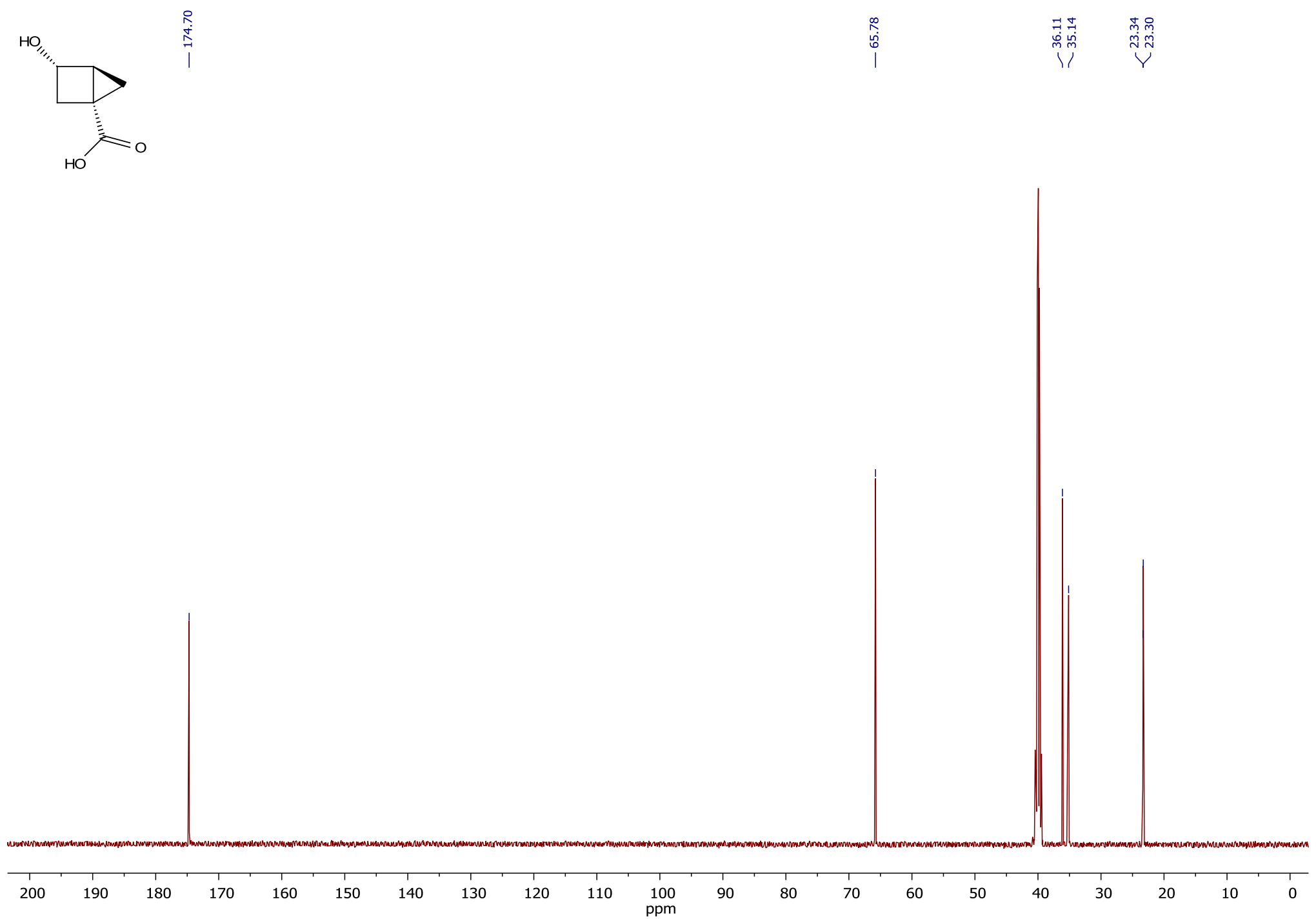

$\left(1 R^{*}, 3 S^{*}, 4 S^{*}\right)-3$-Hydroxybicyclo[2.1.0]pentane-1-carboxylic acid (10g) ${ }^{13} \mathrm{C}$ NMR (DMSO-d $)$ 

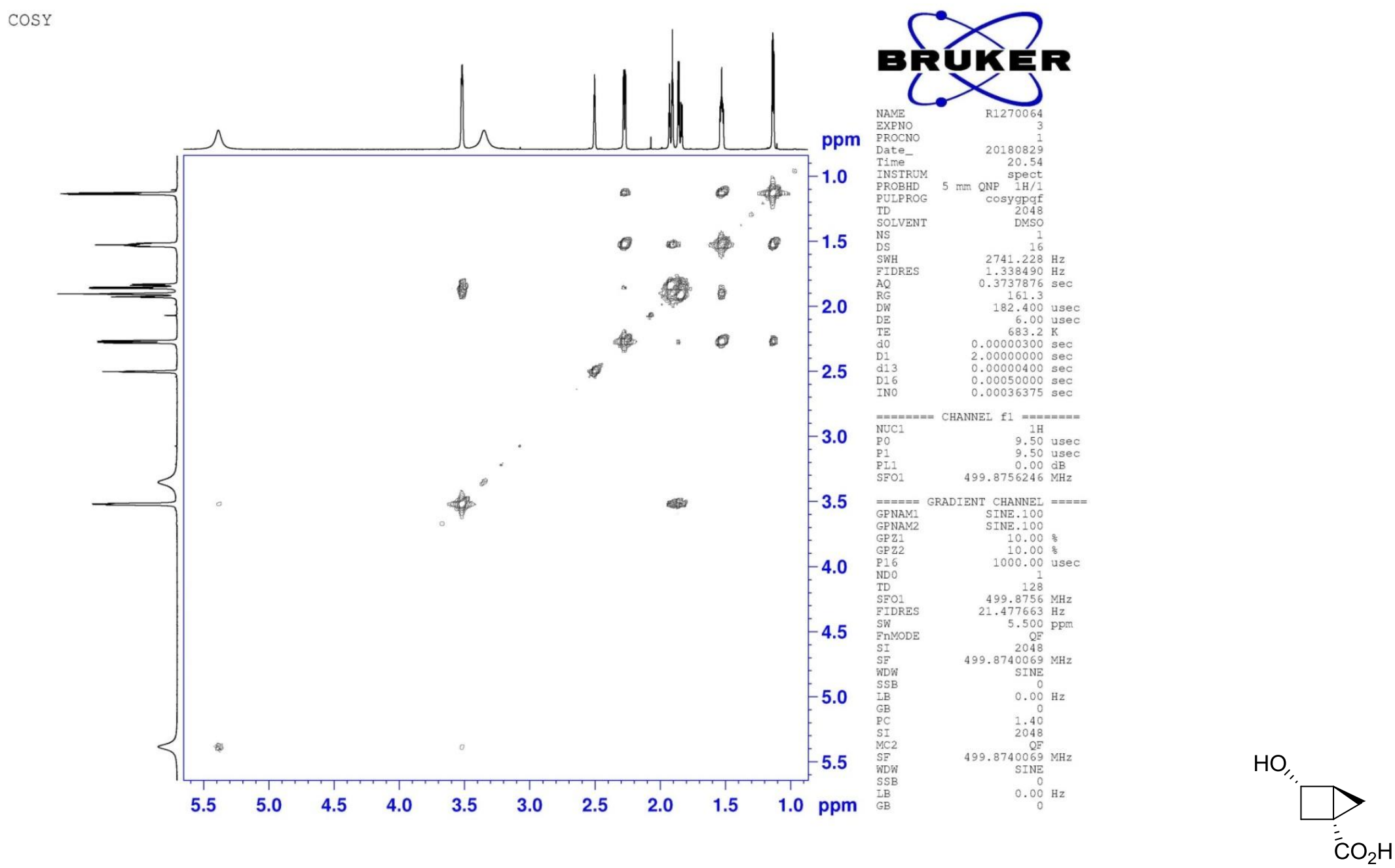

$\left(1 R^{*}, 3 S^{*}, 4 S^{*}\right)$-3-Hydroxybicyclo[2.1.0]pentane-1-carboxylic acid (10g) (COSY) (DMSO-d $\left.{ }_{6}\right)$ 


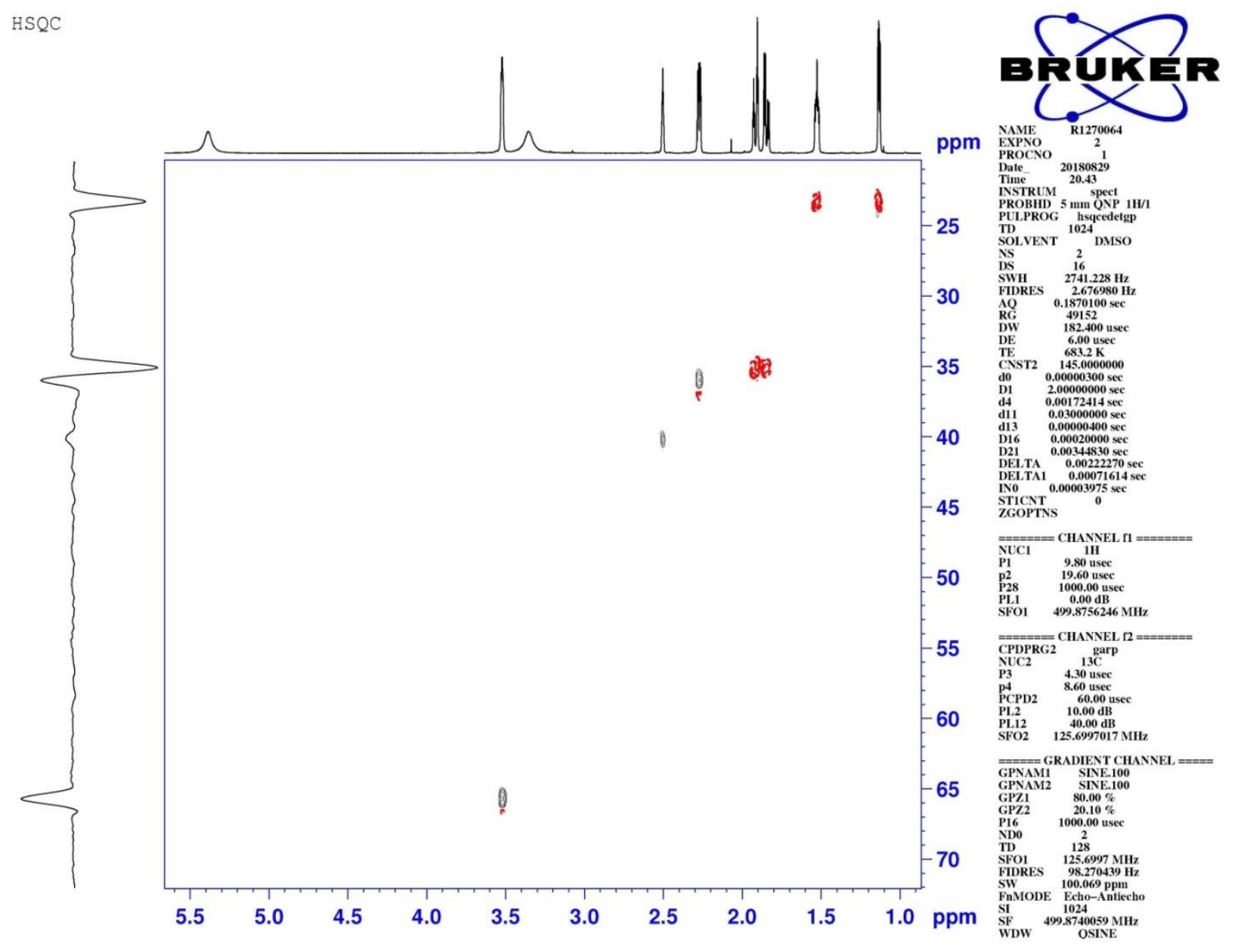

$\mathrm{HO}$,

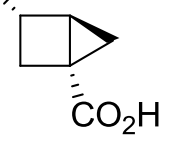

$\left(1 R^{*}, 3 S^{*}, 4 S^{*}\right)-3-H y d r o x y b i c y c l o[2.1 .0]$ pentane-1-carboxylic acid (10g) (HSQC) (DMSO-d $\left.{ }_{6}\right)$ 

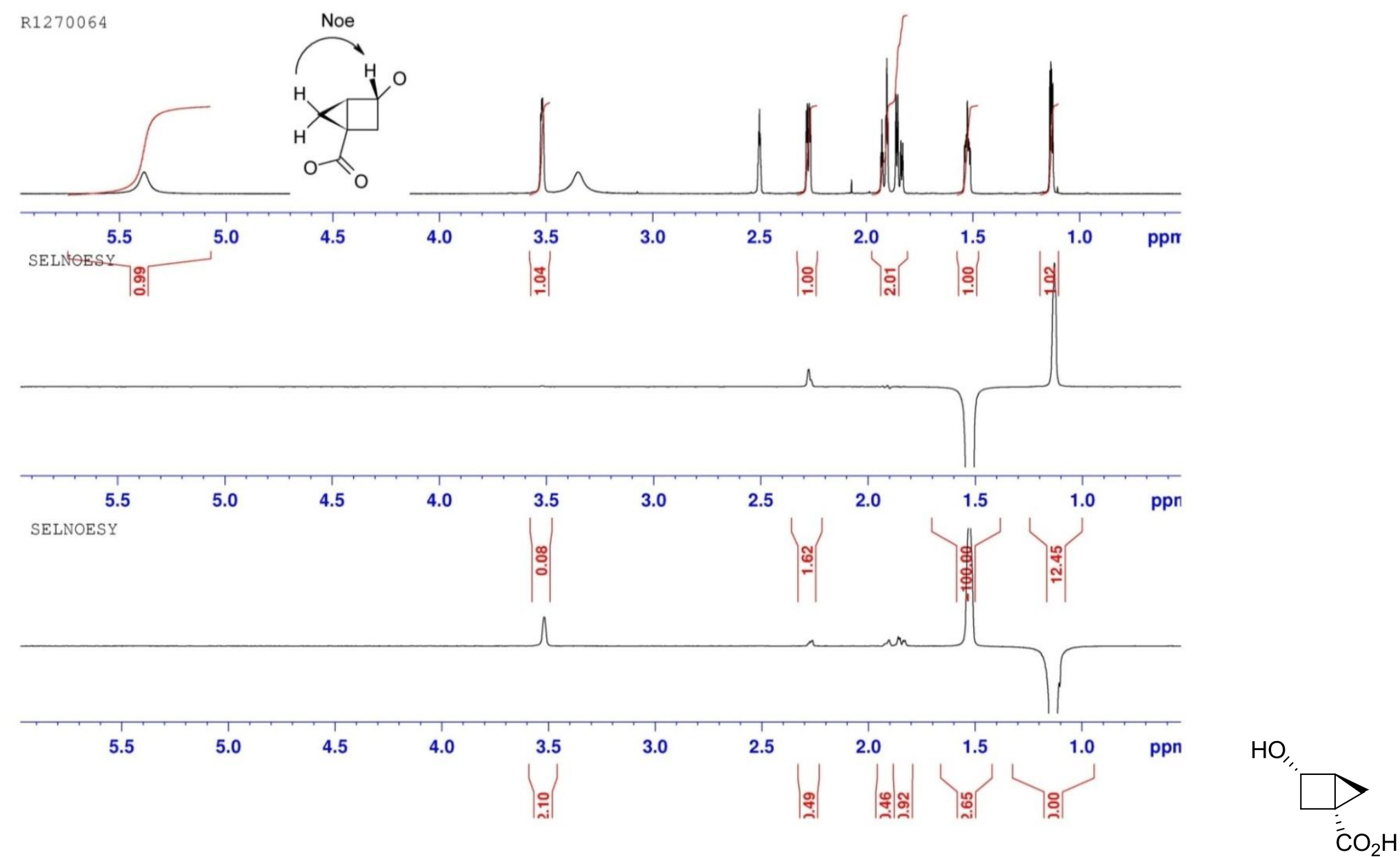

$\left(1 R^{*}, 3 S^{*}, 4 S^{*}\right)-3-H y d r o x y b i c y c l o[2.1 .0]$ pentane-1-carboxylic acid (10g) (H-H NOE) (DMSO-d $)$ 


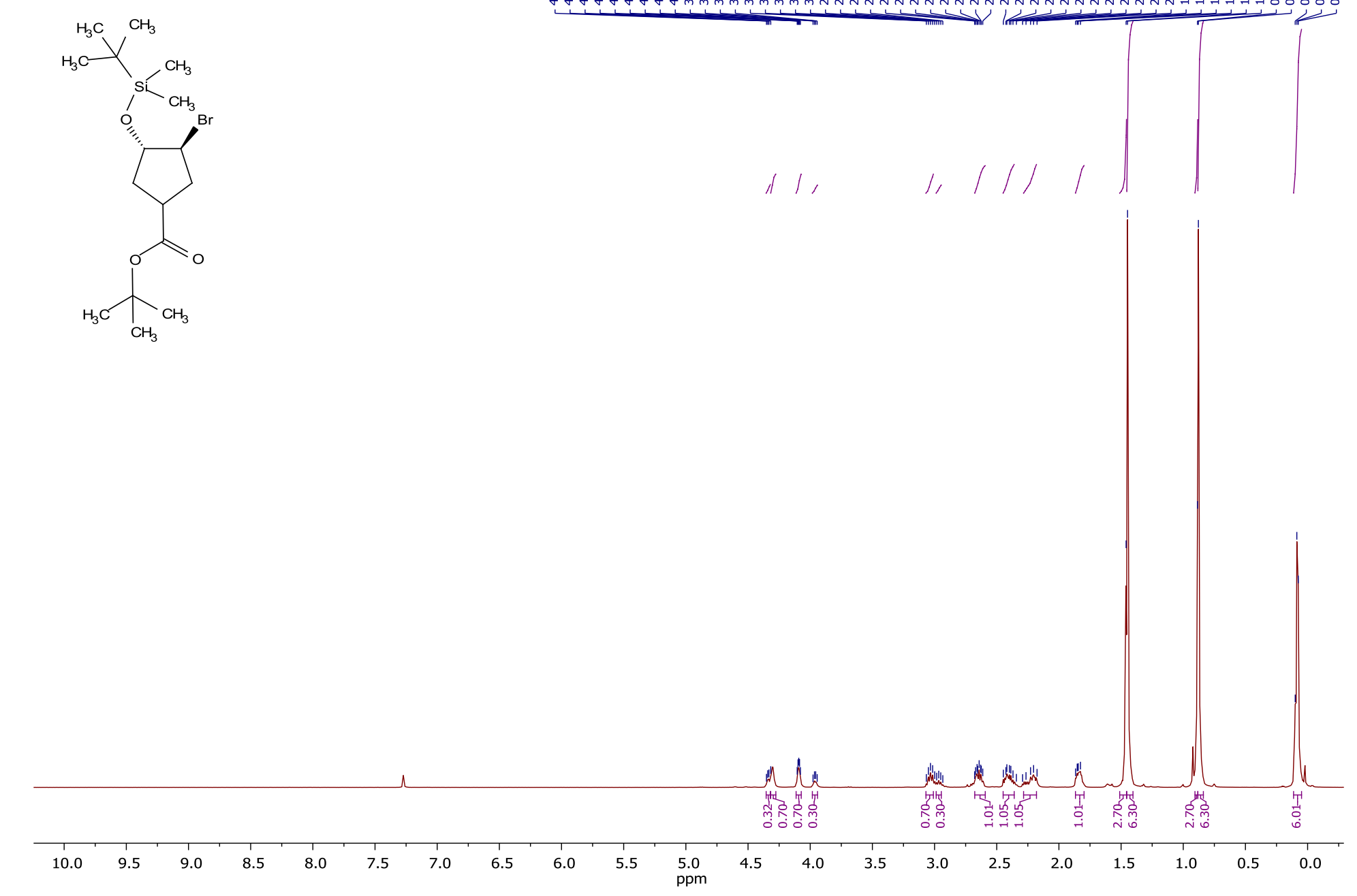

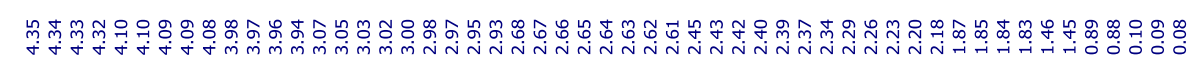

$\left(3 S^{*}, 4 S^{*}\right)$-tert-Butyl 3-bromo-4-((tert-butyldimethylsilyl)oxy)cyclopentanecarboxylate $(11 \mathrm{~g}){ }^{1} \mathrm{H}$ NMR $\left(\mathrm{CDCl}_{3}\right)$ 

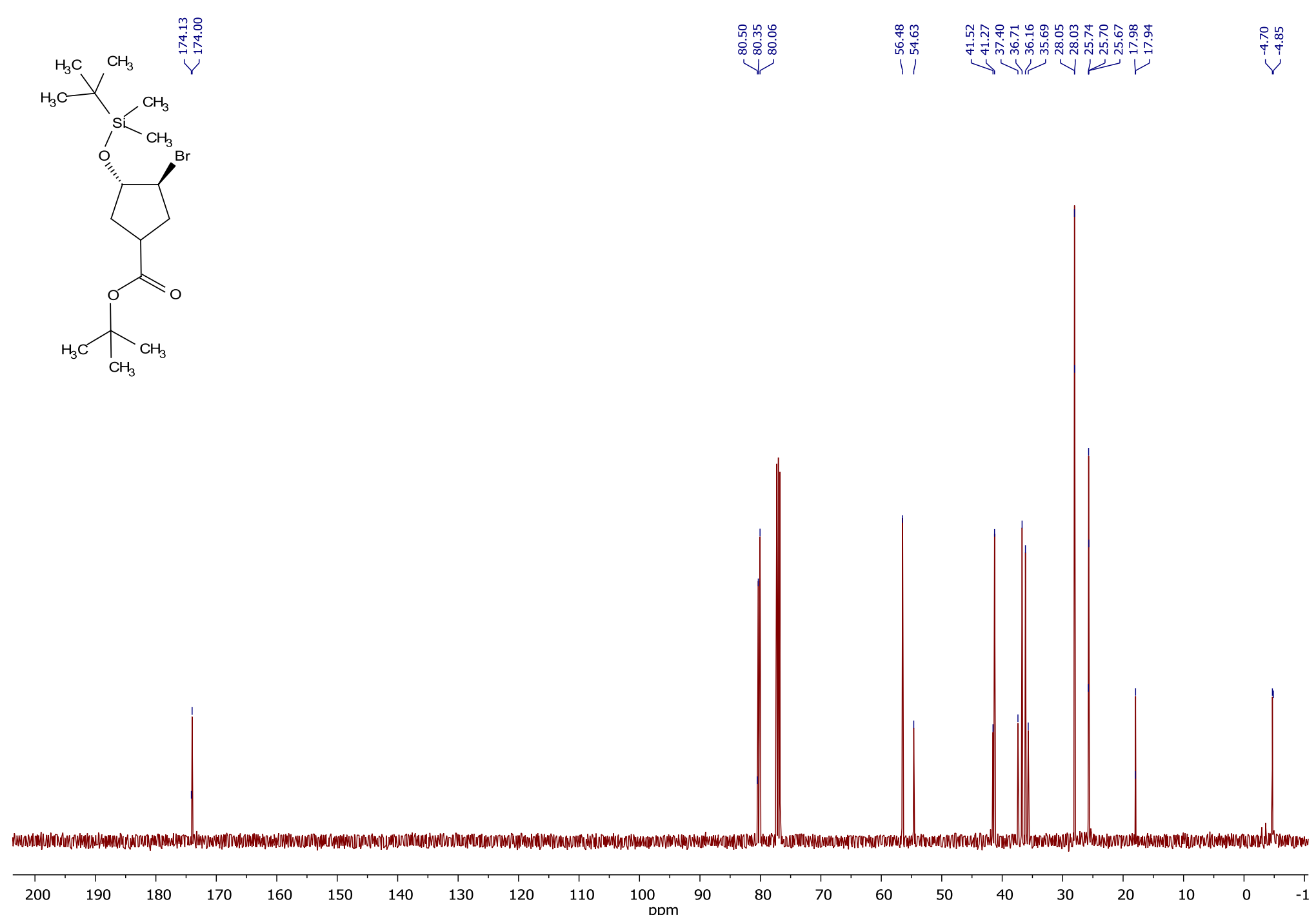

$\left(3 S^{*}, 4 S^{*}\right)$-tert-Butyl 3-bromo-4-((tert-butyldimethylsilyl)oxy)cyclopentanecarboxylate $(11 \mathrm{~g}){ }^{13} \mathrm{C} \mathrm{NMR}\left(\mathrm{CDCl}_{3}\right)$ 

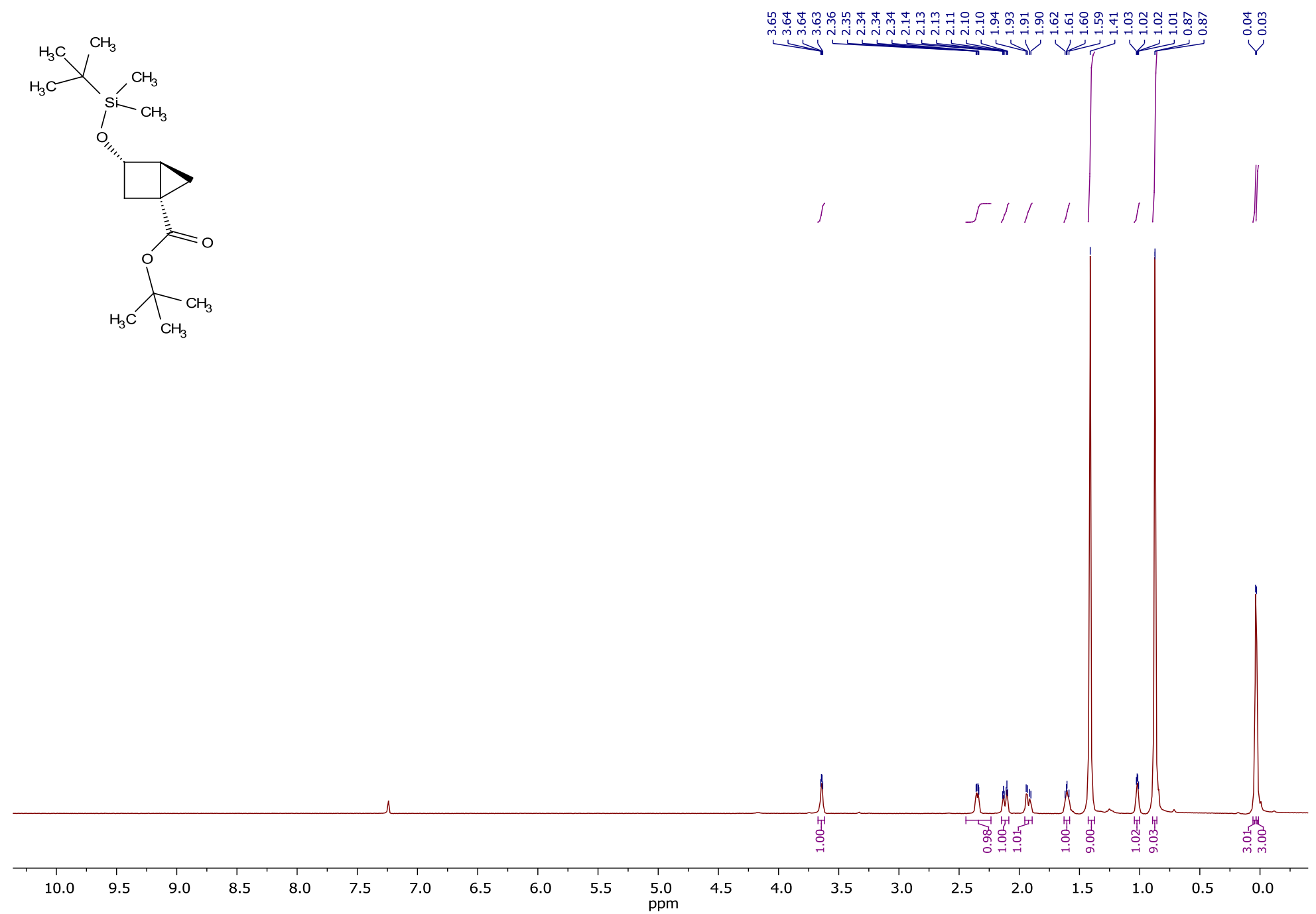

$\left(1 R^{*}, 3 S^{*}, 4 S^{*}\right)$-tert-Butyl 3-((tert-butyldimethylsilyl)oxy)bicyclo[2.1.0]pentane-1-carboxylate (12g) $\left.{ }^{1} \mathrm{H} \mathrm{NMR}_{(\mathrm{CDCl}}\right)$ 


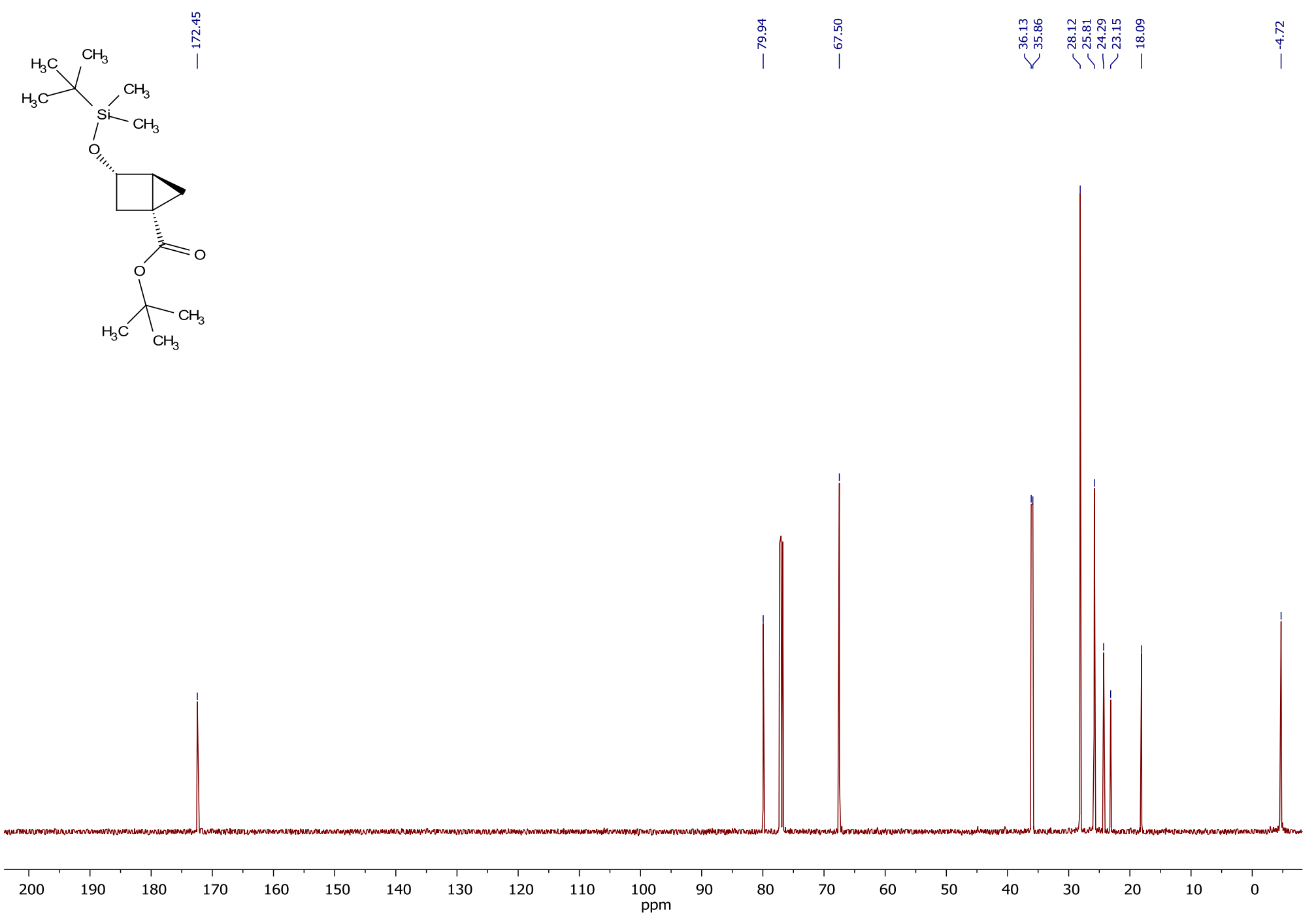

$\left(1 R^{*}, 3 S^{*}, 4 S^{*}\right)$-tert-Butyl 3-((tert-butyldimethylsilyl)oxy)bicyclo[2.1.0]pentane-1-carboxylate (12g) ${ }^{13} \mathrm{C}$ NMR $\left(\mathrm{CDCl}_{3}\right)$ 


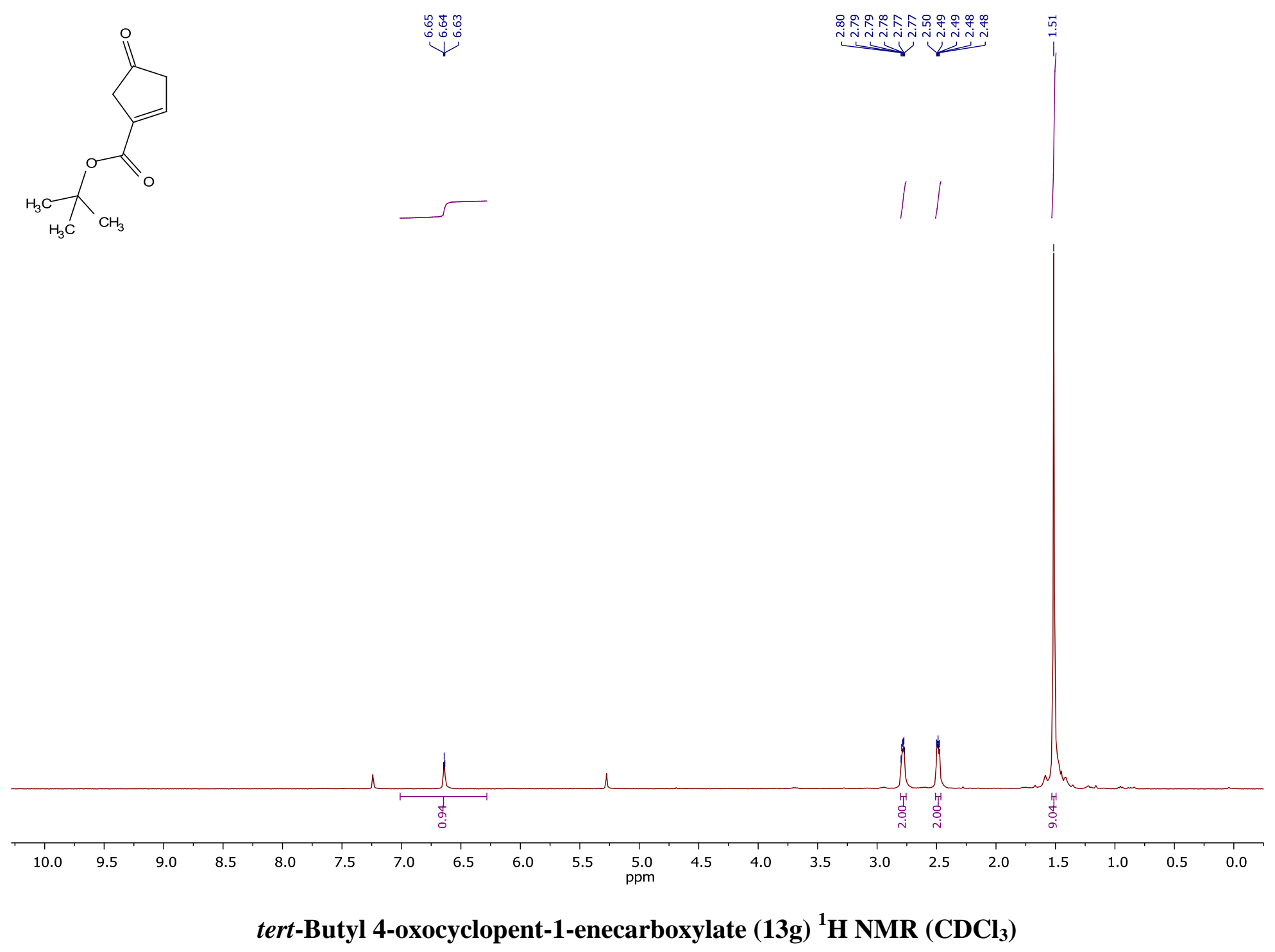




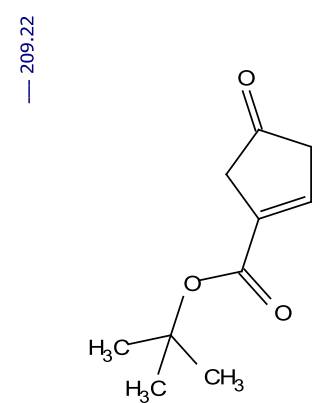

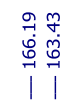

$\mathrm{H}_{3} \mathrm{C} \quad \mathrm{CH}_{3}$

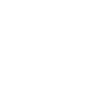

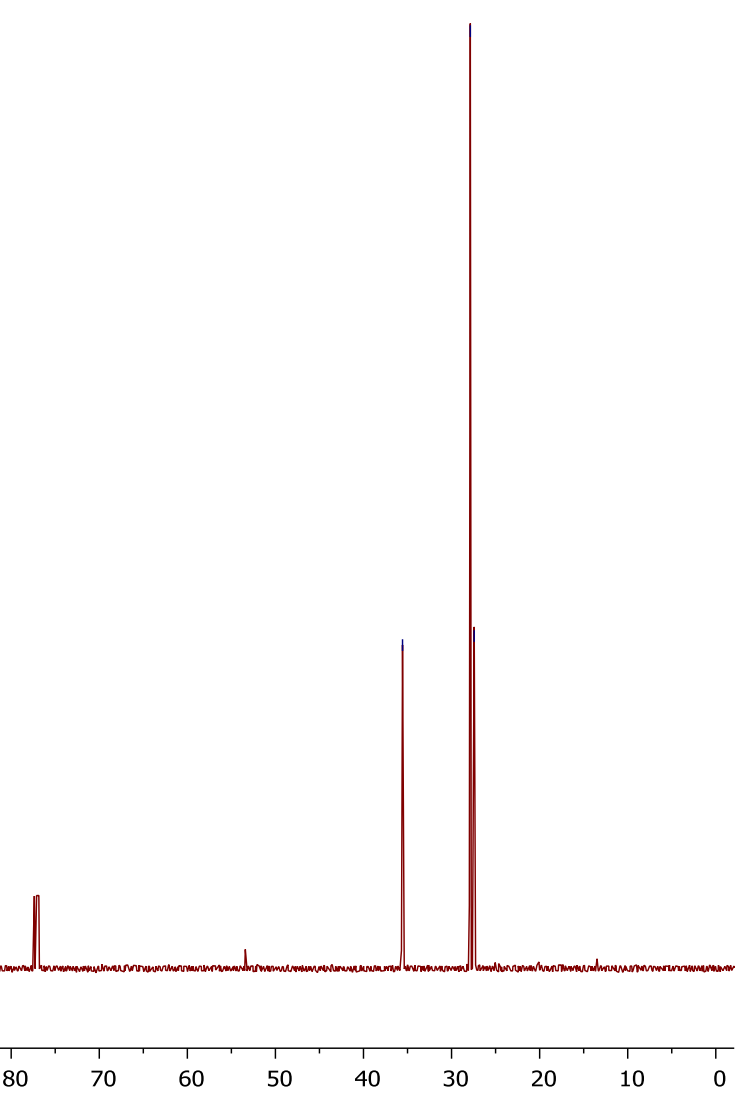

220

$210 \quad 200$

$190 \quad 180$

17 160 140 $130 \quad 120$ 110
$\mathrm{ppm}$

tert-Butyl 4-oxocyclopent-1-enecarboxylate (13g) ${ }^{13} \mathrm{C} \mathrm{NMR}\left(\mathrm{CDCl}_{3}\right)$ 

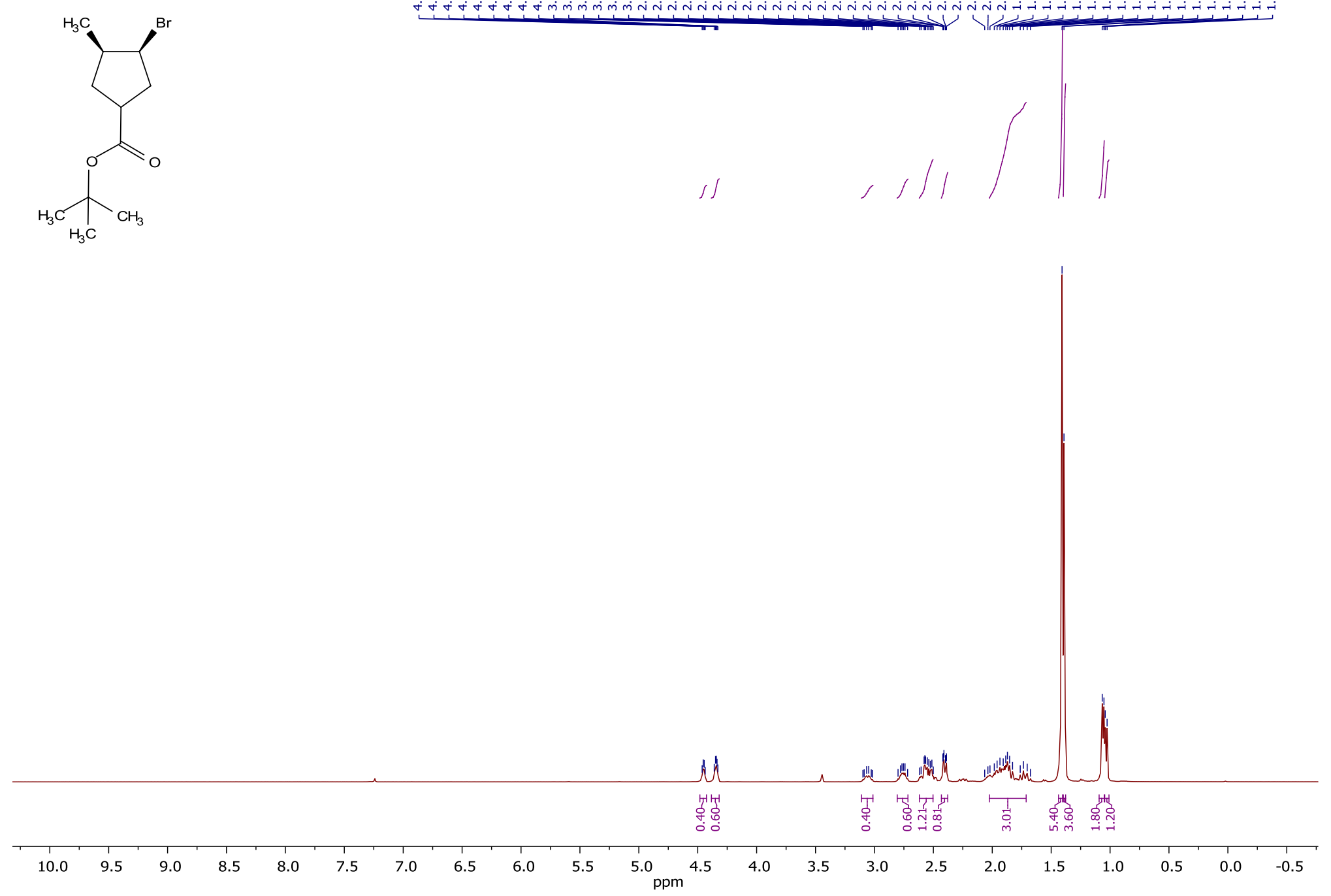

$\left(3 S^{*}, 4 R^{*}\right)$-tert-Butyl 3-bromo-4-methylcyclopentanecarboxylate (15a) ${ }^{1} \mathrm{H}$ NMR $\left(\mathrm{CDCl}_{3}\right)$ 

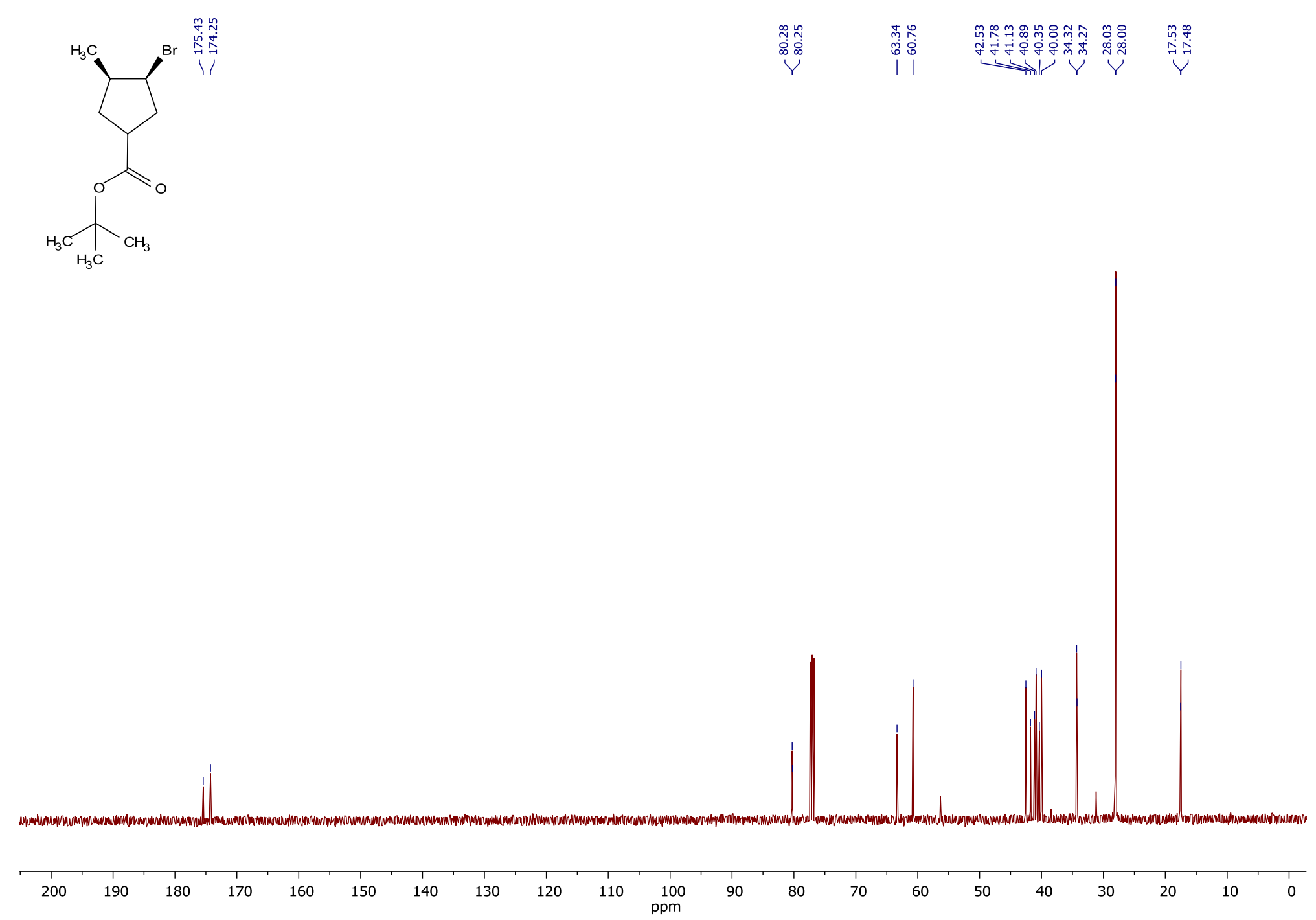

$\left(3 S^{*}, 4 R^{*}\right)$-tert-Butyl 3-bromo-4-methylcyclopentanecarboxylate (15a) ${ }^{13} \mathrm{C} \mathrm{NMR}\left(\mathrm{CDCl}_{3}\right)$ 


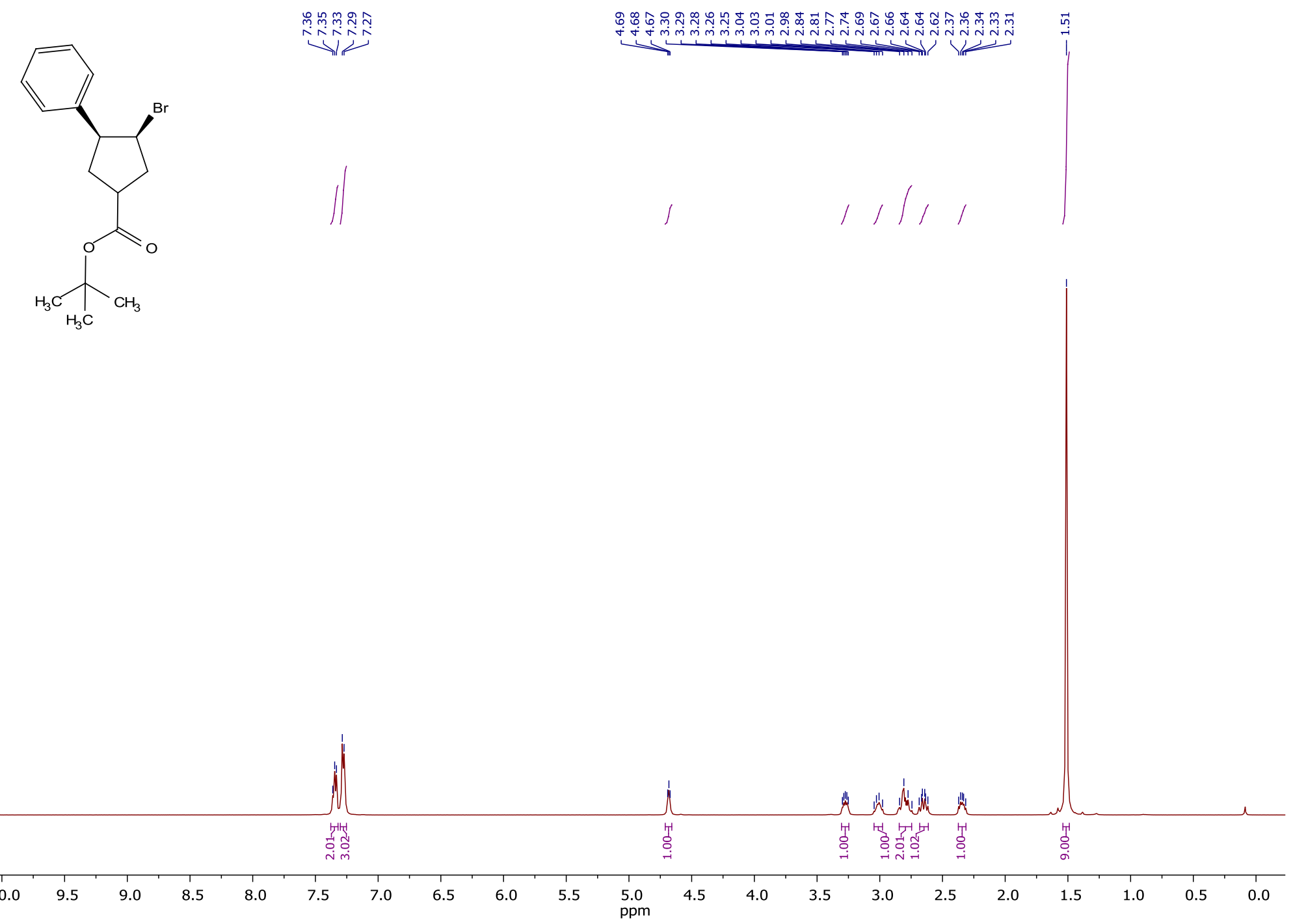

$\left(3 S^{*}, 4 S^{*}\right)$-tert-Butyl 3-bromo-4-phenylcyclopentanecarboxylate (15b) ${ }^{1} \mathrm{H} \mathrm{NMR}\left(\mathrm{CDCl}_{3}\right)$ 


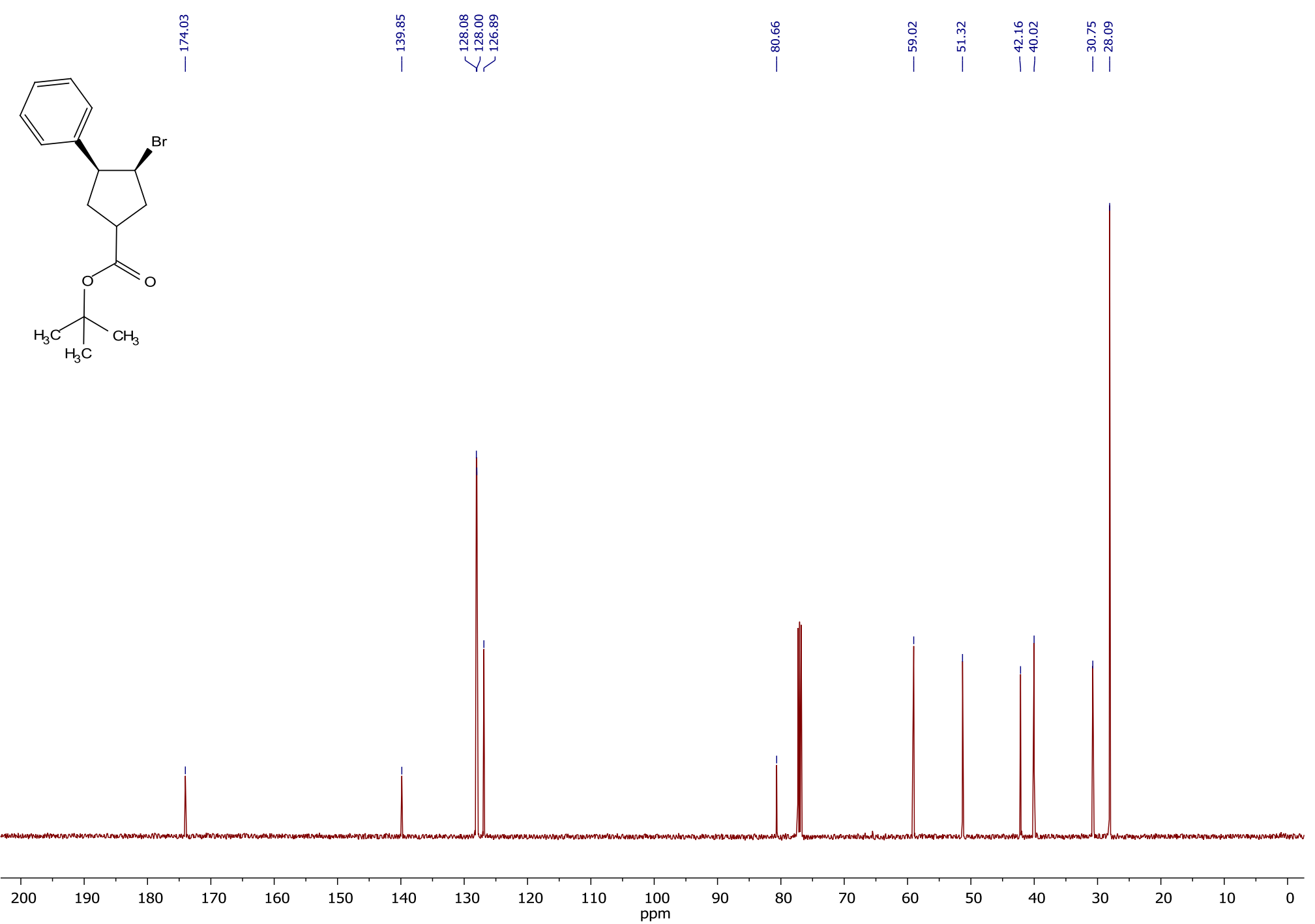

$\left(3 S^{*}, 4 S^{*}\right)$-tert-Butyl 3-bromo-4-phenylcyclopentanecarboxylate $(15 \mathrm{~b}){ }^{13} \mathrm{C} \mathrm{NMR}\left(\mathrm{CDCl}_{3}\right)$ 


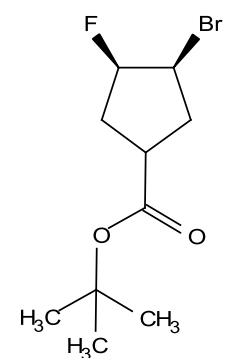

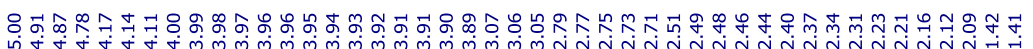
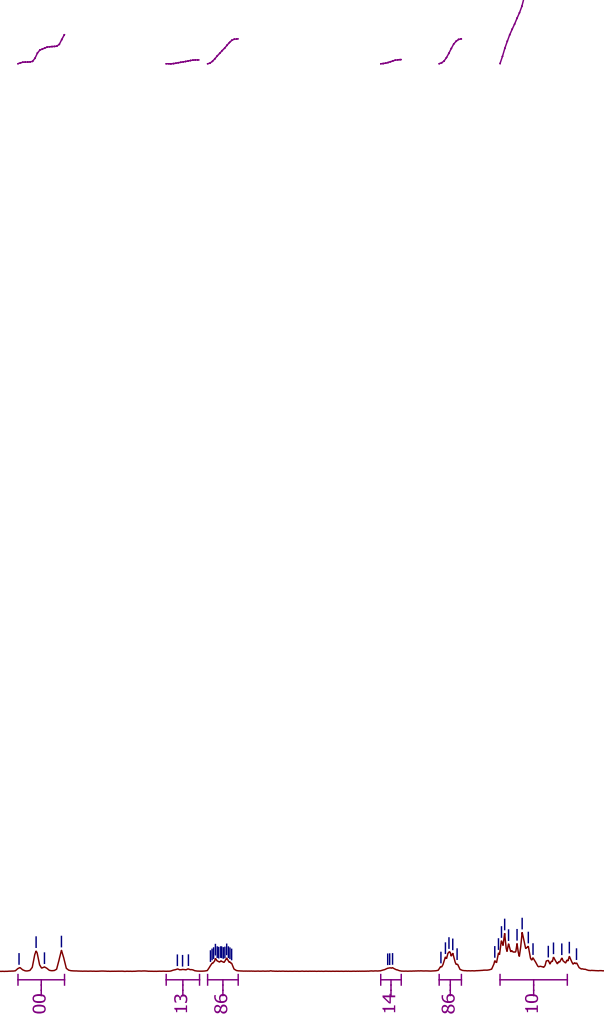

10.0

$\left(3 S^{*}, 4 R^{*}\right)$-tert-Butyl 3-bromo-4-fluorocyclopentanecarboxylate (15c) ${ }^{1} \mathrm{H} \mathrm{NMR}\left(\mathrm{CDCl}_{3}\right)$ 

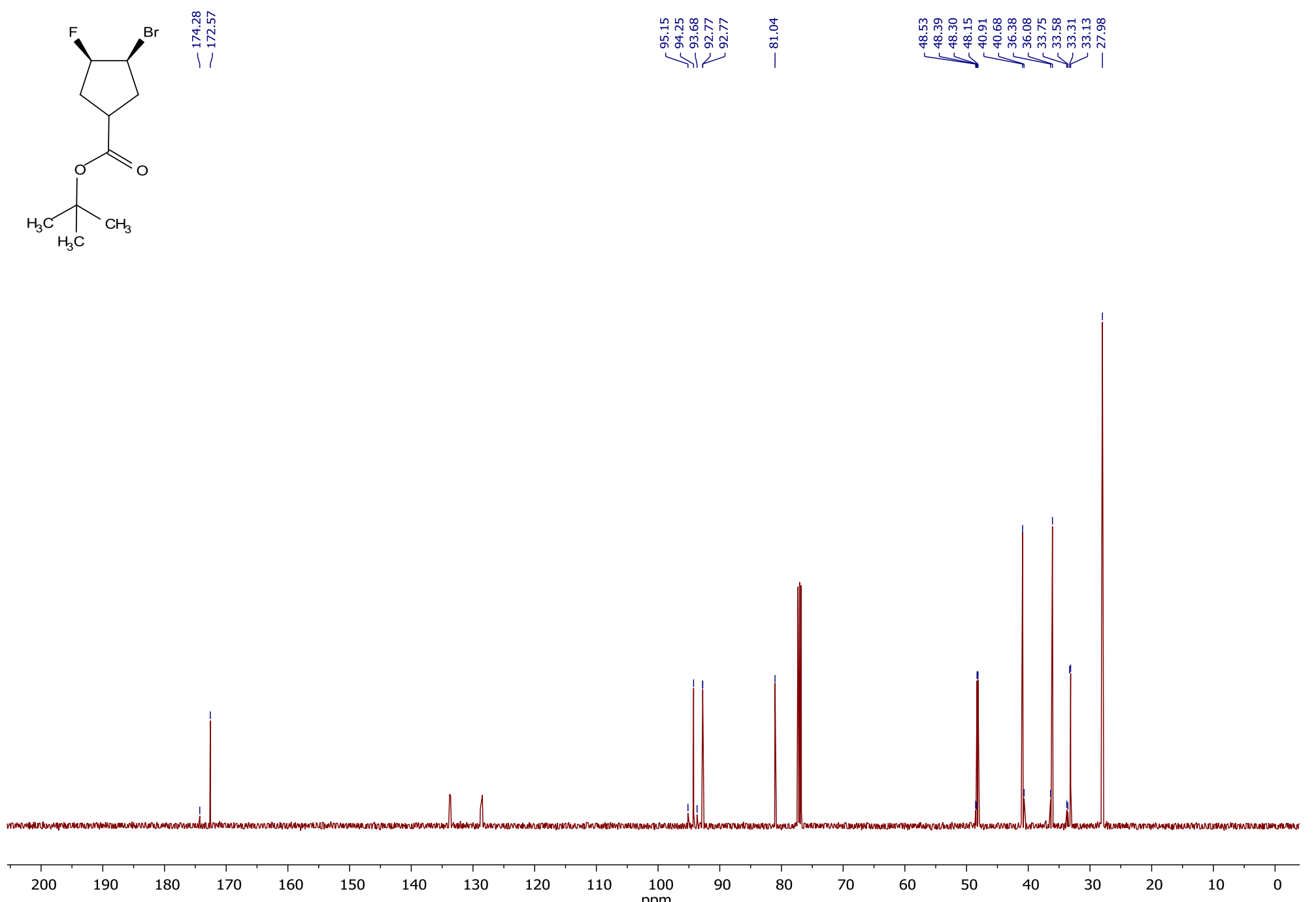

$\left(3 S^{*}, 4 R^{*}\right)$-tert-Butyl 3-bromo-4-fluorocyclopentanecarboxylate $(15 \mathrm{c}){ }^{13} \mathrm{C} \mathrm{NMR}\left(\mathrm{CDCl}_{3}\right)$ 

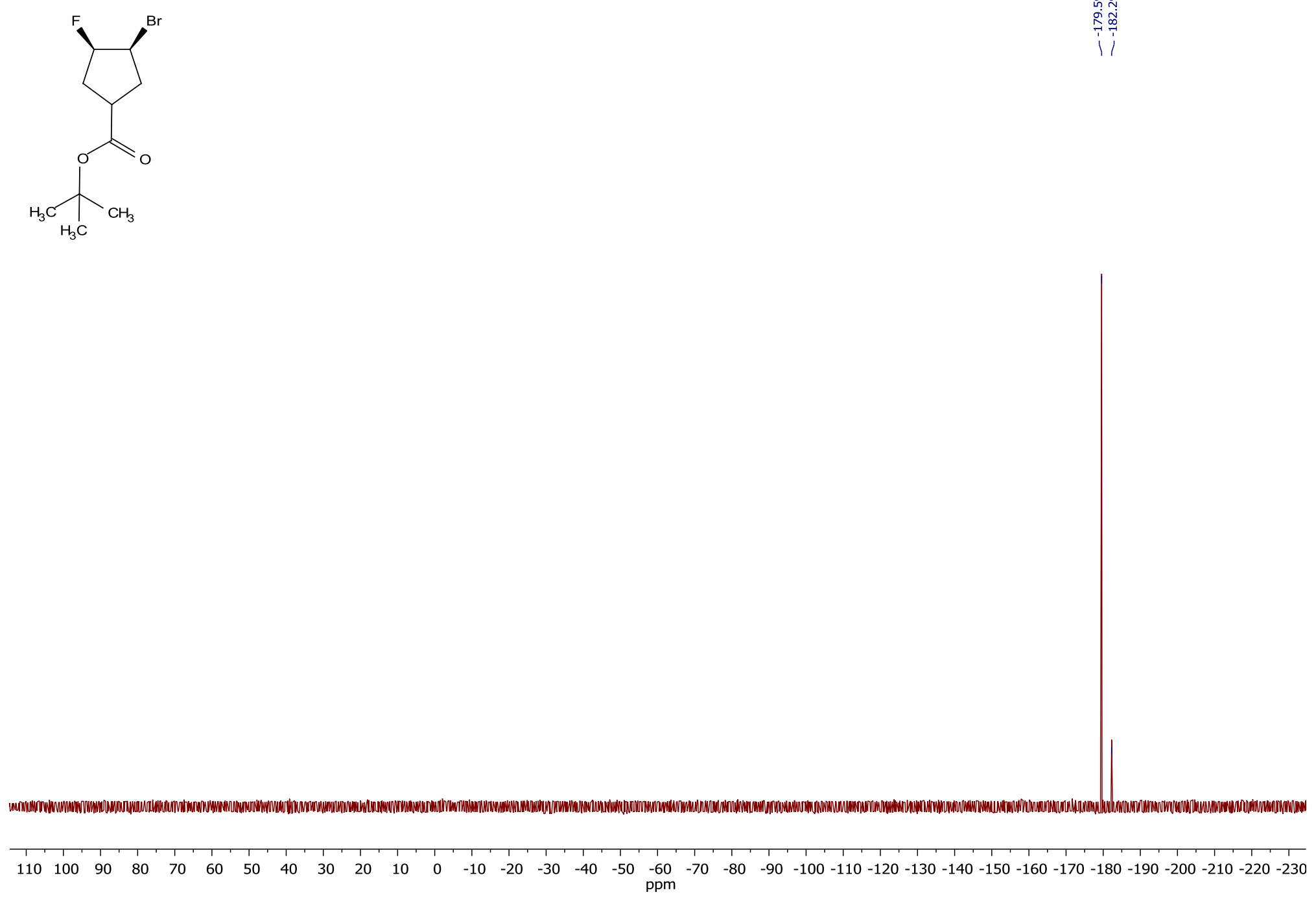
$\left(3 S^{*}, 4 R^{*}\right)$-tert-Butyl 3-bromo-4-fluorocyclopentanecarboxylate $(15 \mathrm{c}){ }^{19} \mathrm{~F} \mathrm{NMR}\left(\mathrm{CDCl}_{3}\right)$ 

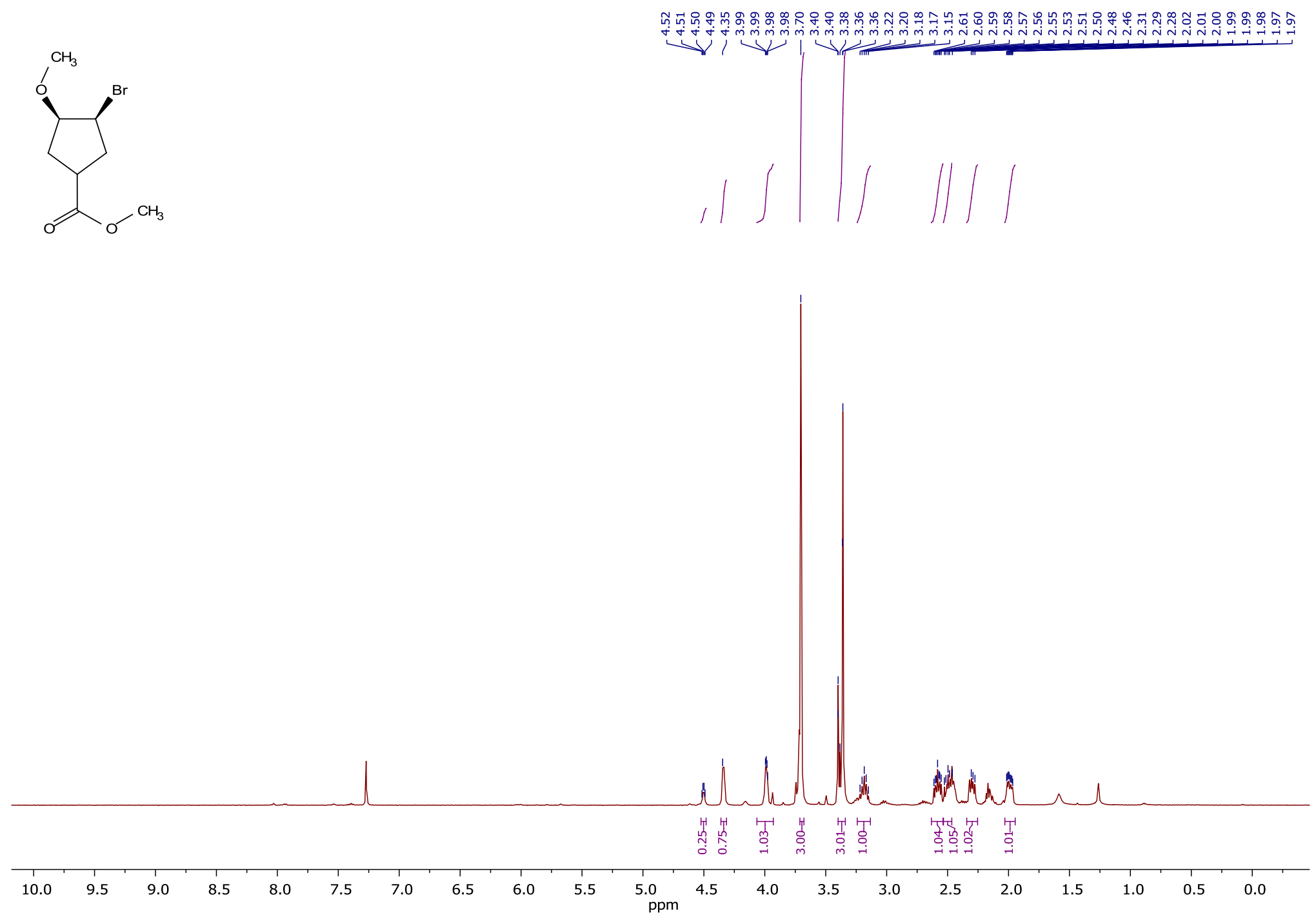

$\left(3 S^{*}, 4 R^{*}\right)$-methyl 3-bromo-4-methoxycyclopentanecarboxylate (15d) ${ }^{1} \mathrm{H} \mathrm{NMR}\left(\mathrm{CDCl}_{3}\right)$ 

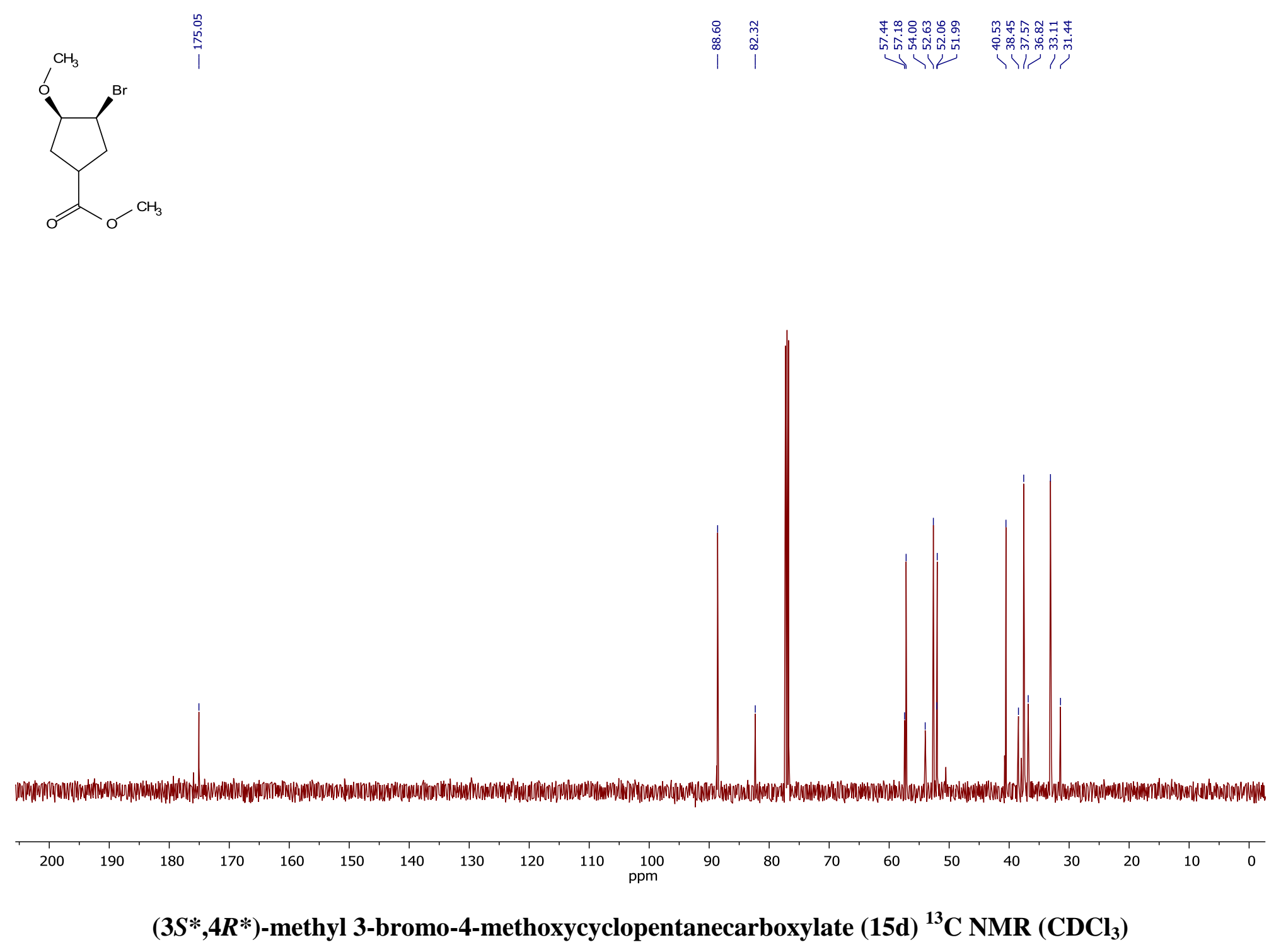


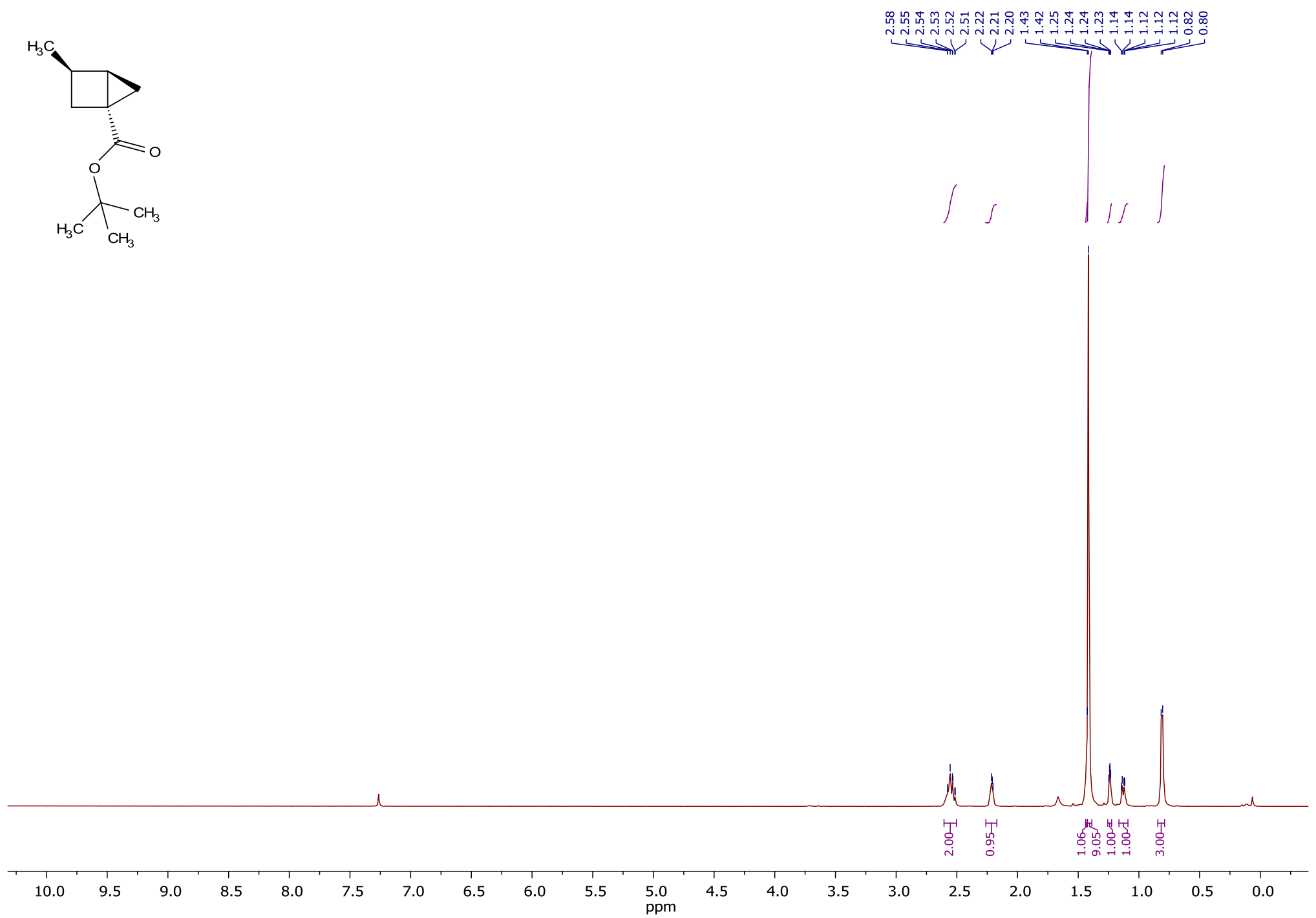

$\left(1 R^{*}, 3 R^{*}, 4 R^{*}\right)$-tert-Butyl 3-methylbicyclo[2.1.0]pentane-1-carboxylate (16a) ${ }^{1} \mathrm{H}$ NMR $\left(\mathrm{CDCl}_{3}\right)$ 


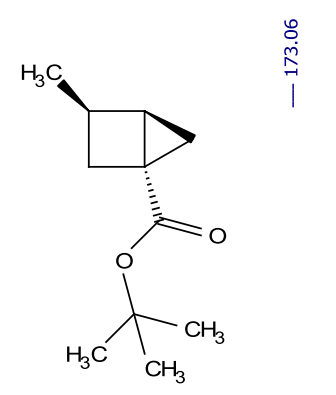

$\overbrace{\mathrm{H}_{3} \mathrm{C}} \mathrm{CH}_{3}$

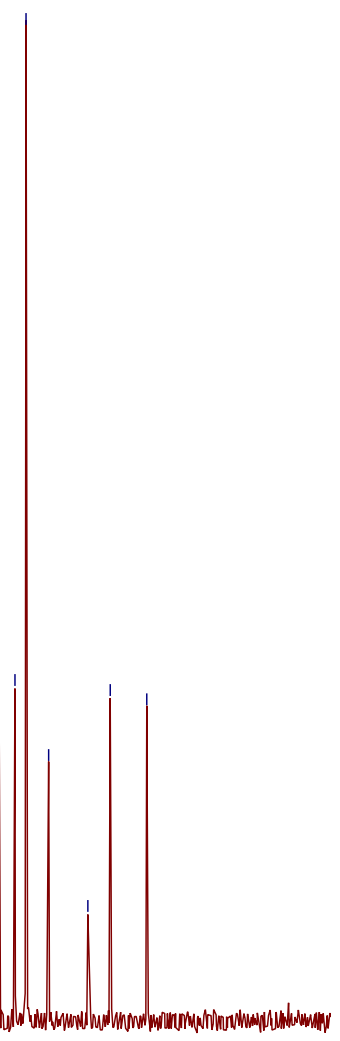

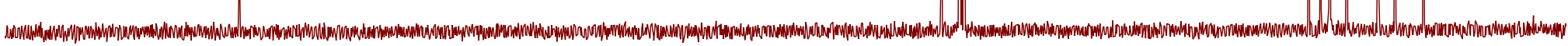

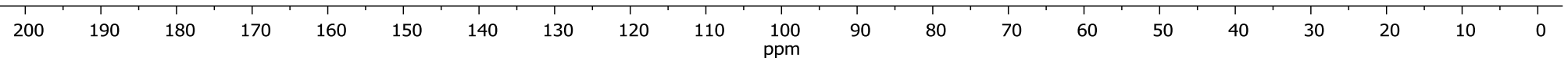

$\left(1 R^{*}, 3 R^{*}, 4 R^{*}\right)$-tert-Butyl 3-methylbicyclo[2.1.0]pentane-1-carboxylate (16a) ${ }^{13} \mathrm{C} \mathrm{NMR}\left(\mathrm{CDCl}_{3}\right)$ 

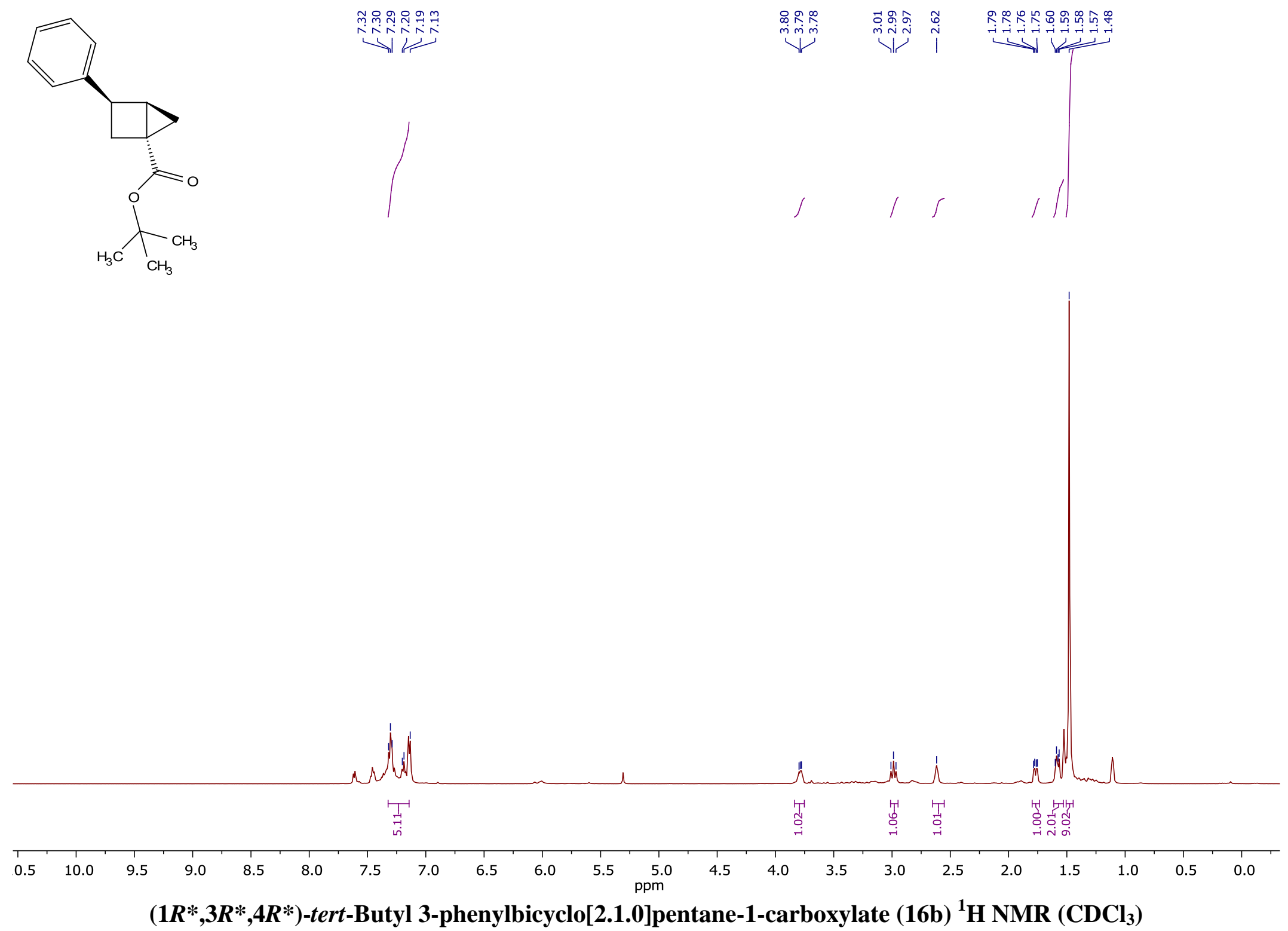


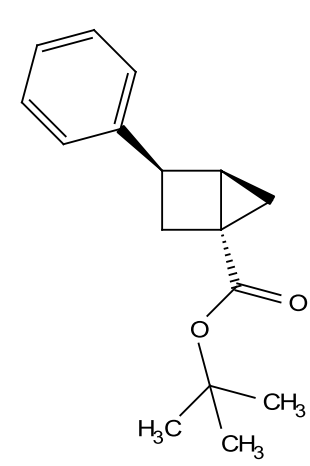

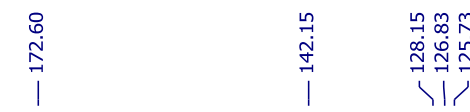

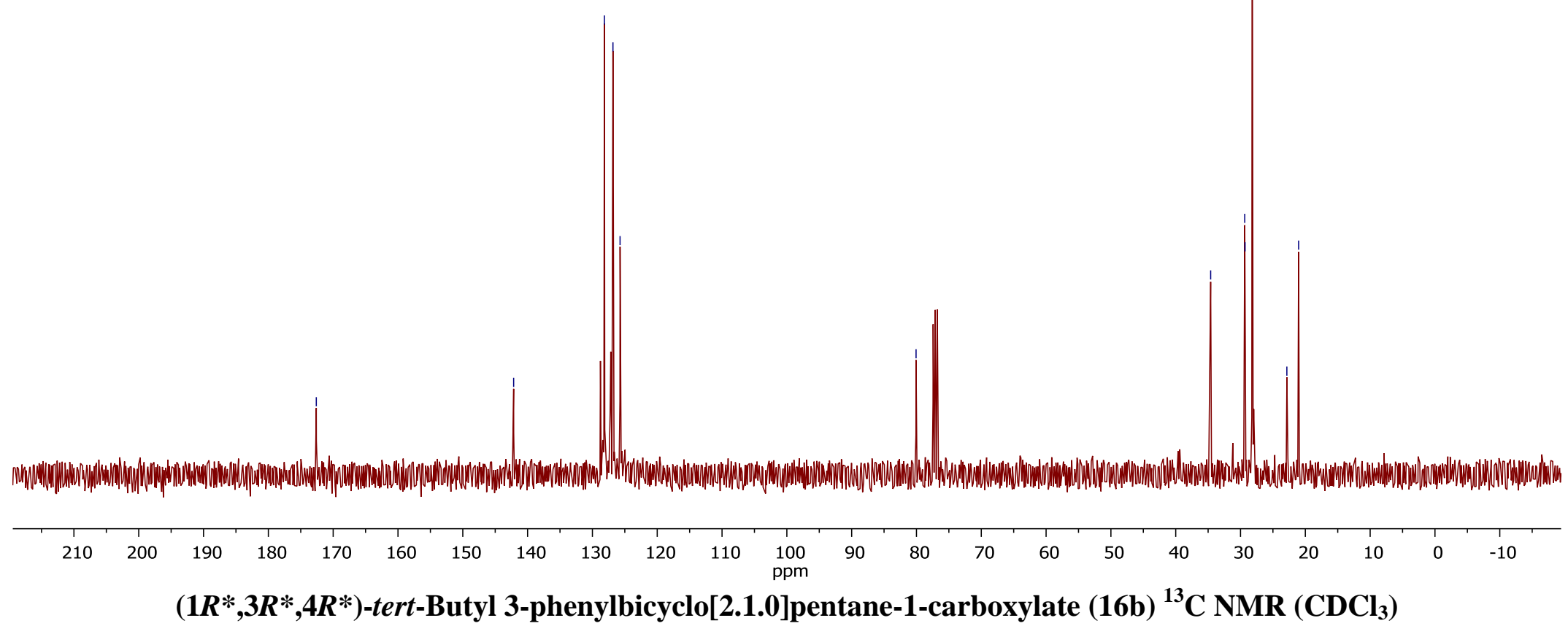




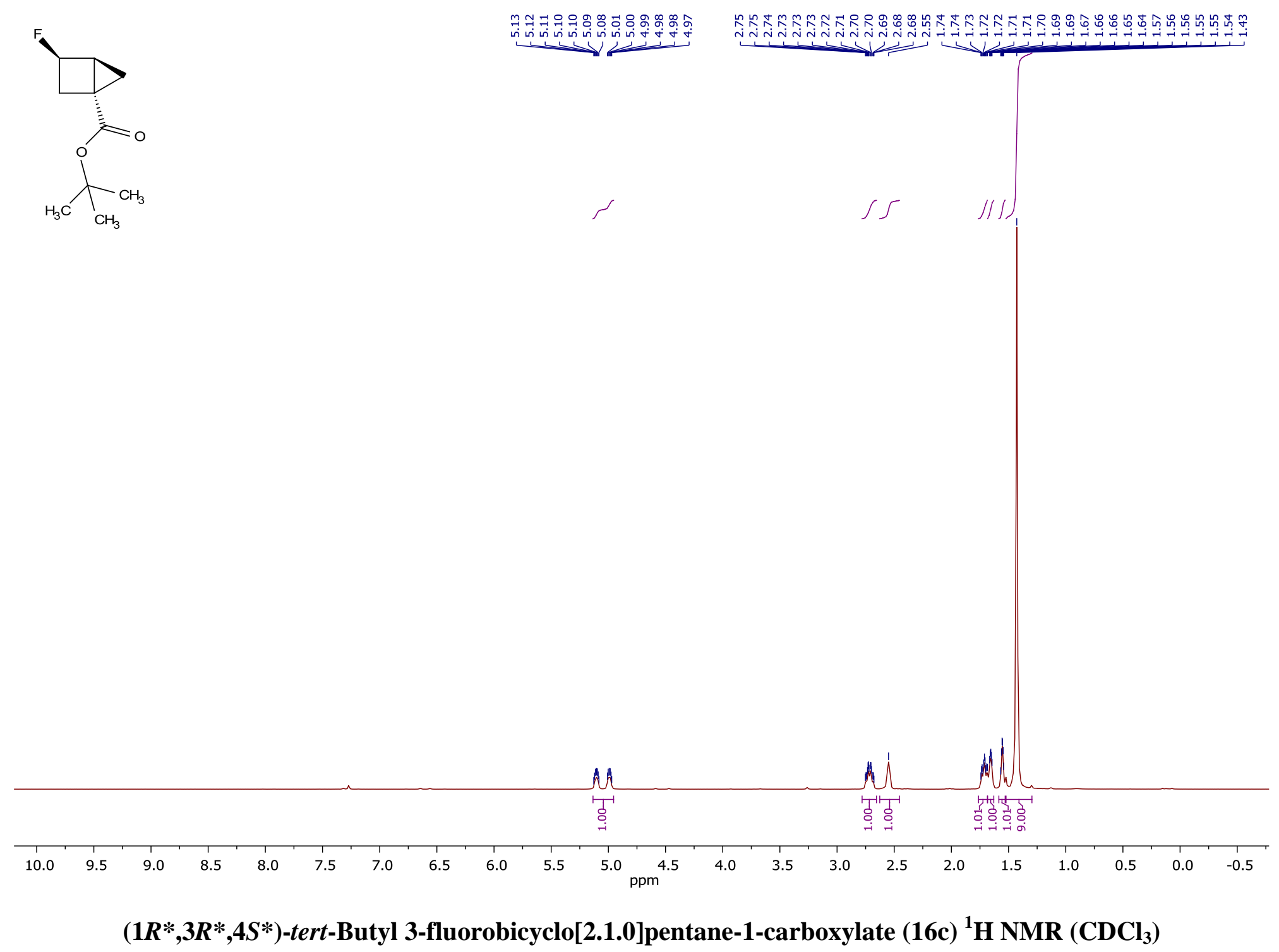




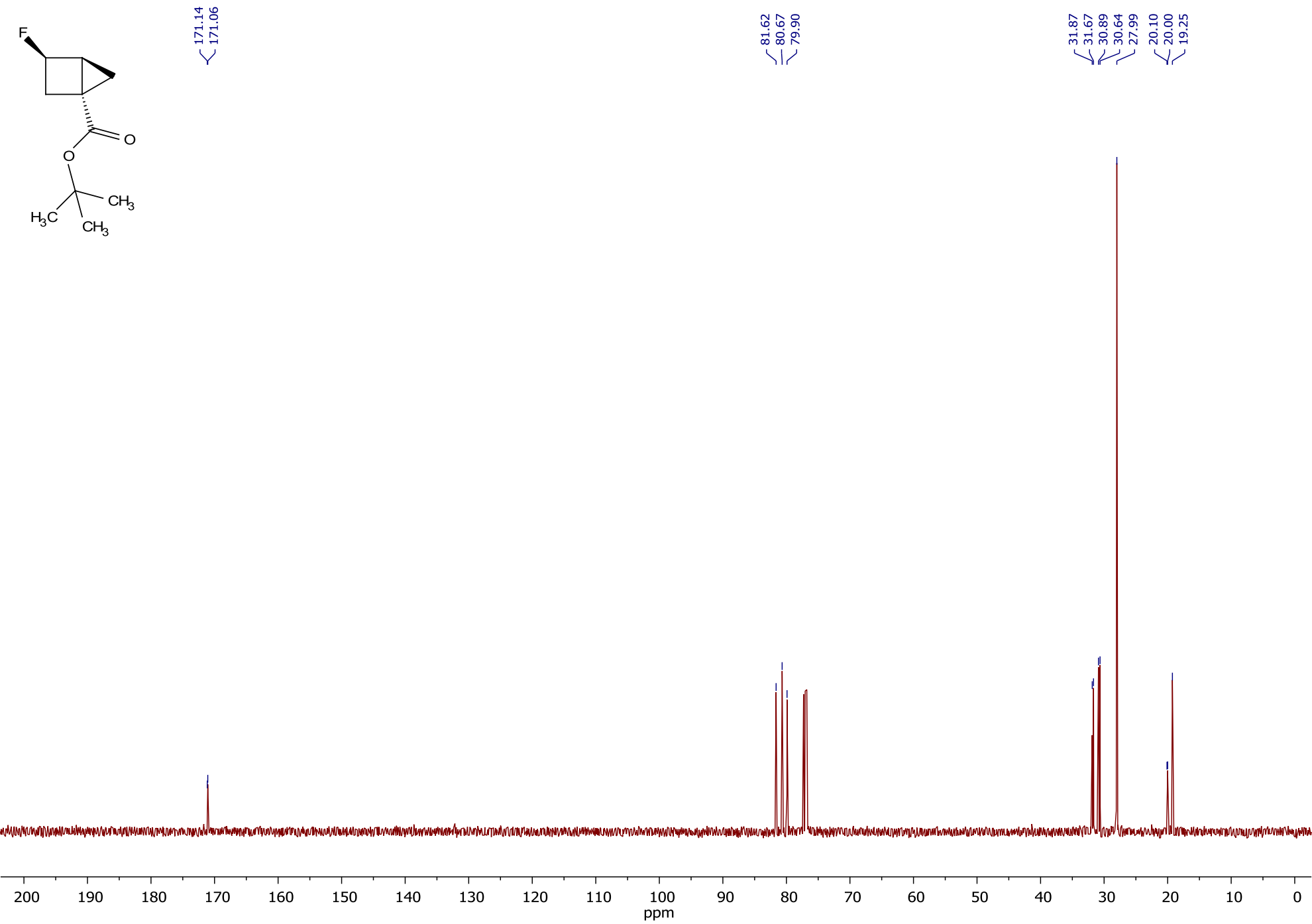

$\left(1 R^{*}, 3 R^{*}, 4 S^{*}\right)$-tert-Butyl 3-fluorobicyclo[2.1.0]pentane-1-carboxylate $(16 \mathrm{c}){ }^{13} \mathrm{C} \mathrm{NMR}\left(\mathrm{CDCl}_{3}\right)$ 


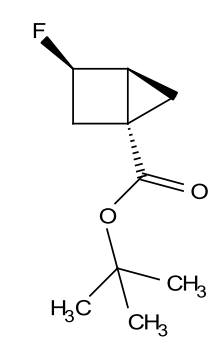

\section{$\left(1 R^{*}, 3 R^{*}, 4 S^{*}\right)$-tert-Butyl 3-fluorobicyclo[2.1.0]pentane-1-carboxylate (16c) ${ }^{19} \mathrm{~F} \mathrm{NMR}\left(\mathrm{CDCl}_{3}\right)$}




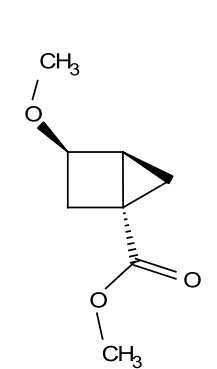

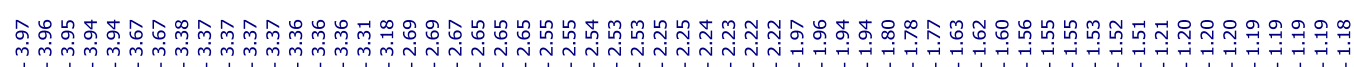

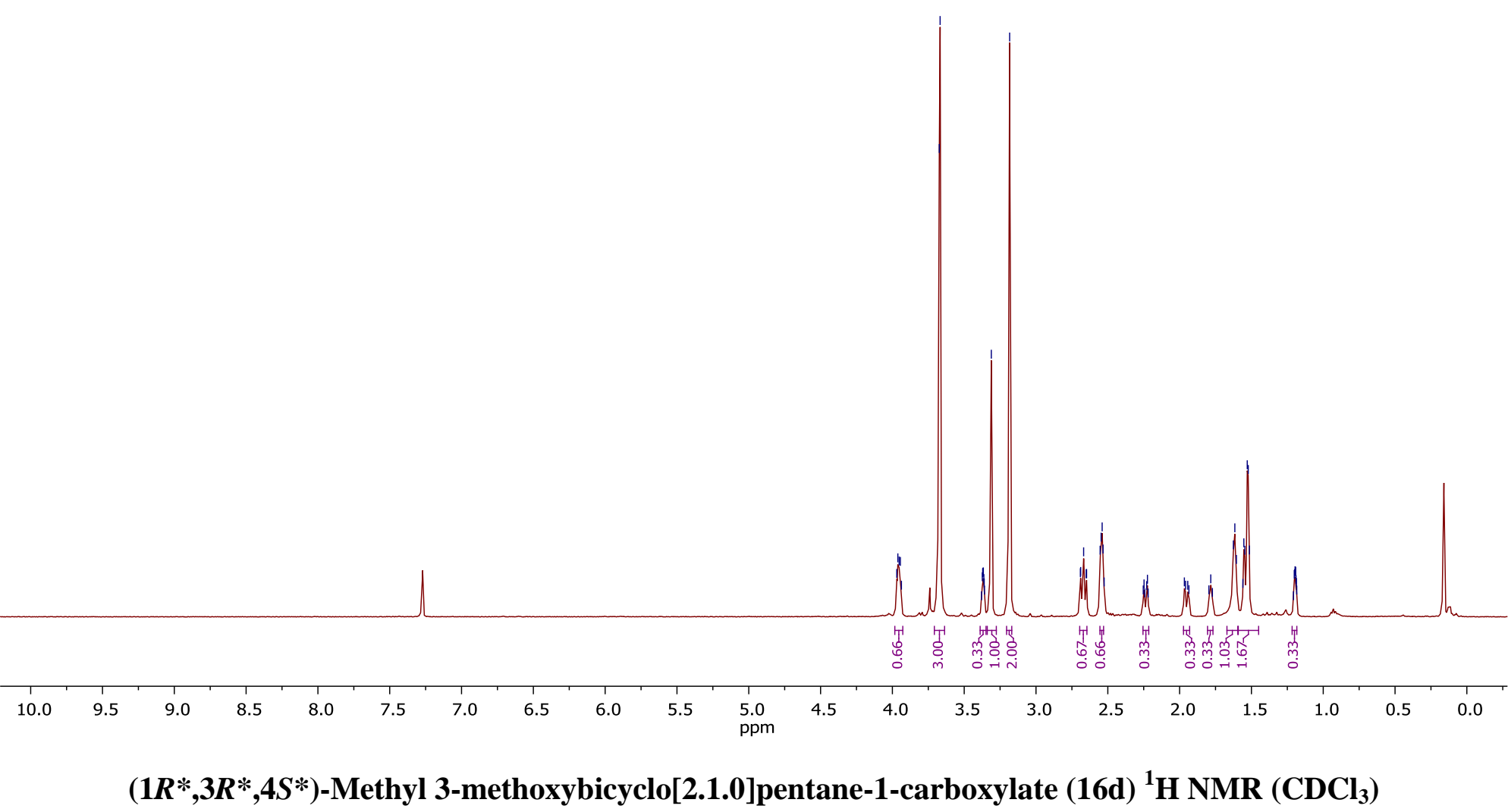




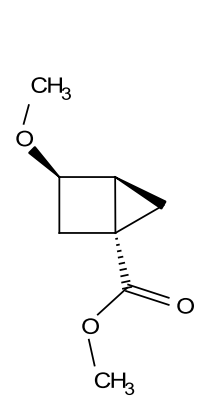

|

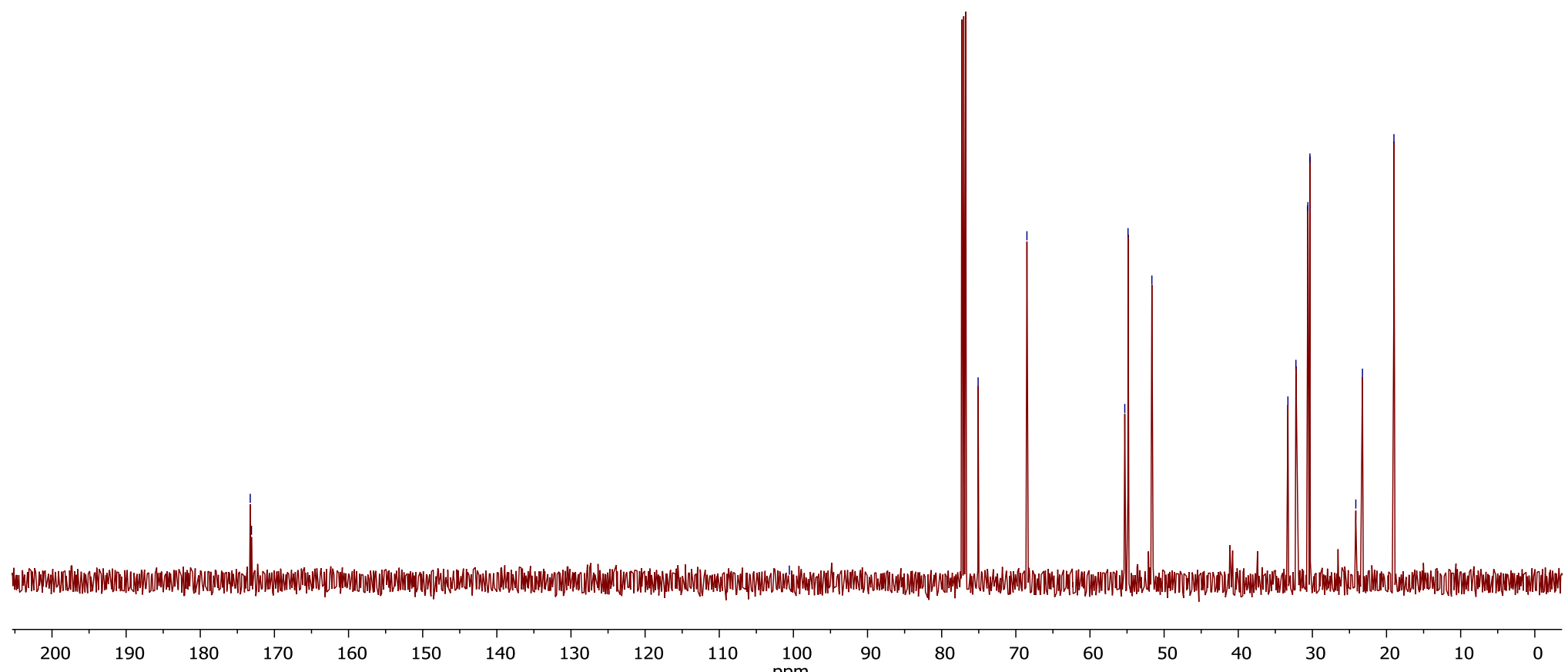

$\left(1 R^{*}, 3 R^{*}, 4 S^{*}\right)$-Methyl 3-methoxybicyclo[2.1.0]pentane-1-carboxylate (16d) ${ }^{13} \mathrm{C} \mathrm{NMR}\left(\mathrm{CDCl}_{3}\right)$ 

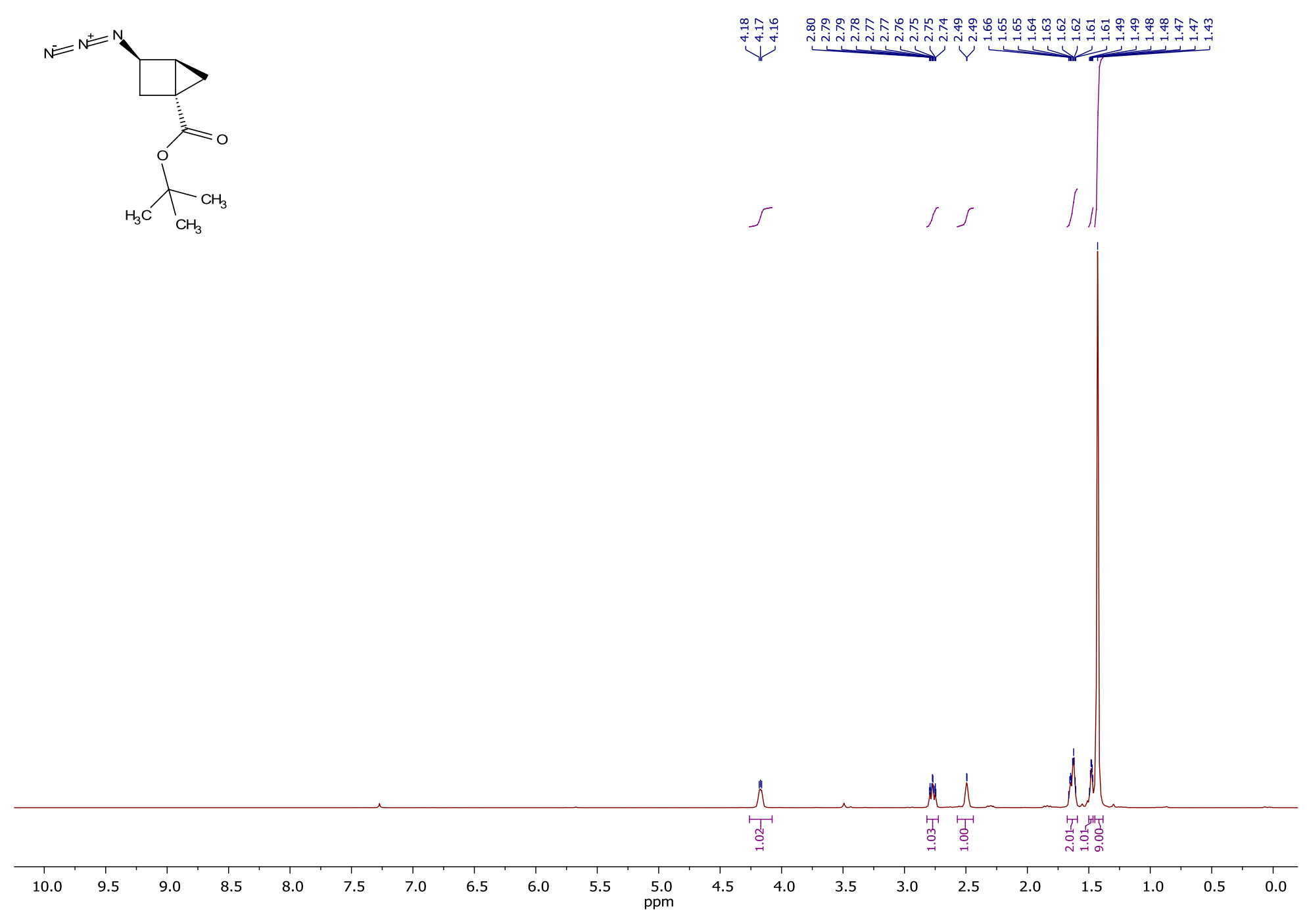

$\left(1 R^{*}, 3 R^{*}, 4 S^{*}\right)$-tert-Butyl 3-azidobicyclo[2.1.0]pentane-1-carboxylate (16f) ${ }^{1} \mathrm{H}$ NMR $\left(\mathrm{CDCl}_{3}\right)$ 

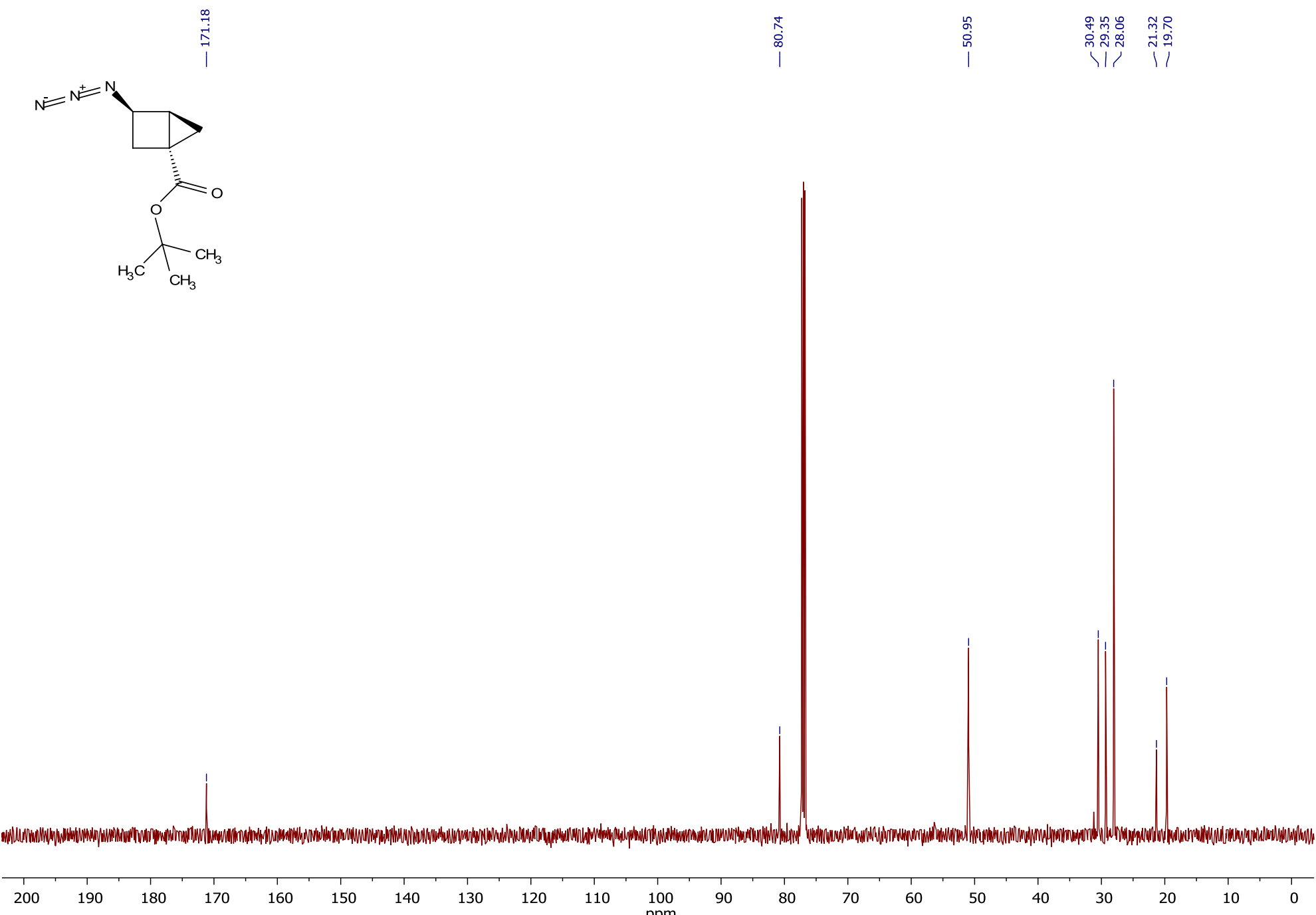

$\left(1 R^{*}, 3 R^{*}, 4 S^{*}\right)$-tert-Butyl 3-azidobicyclo[2.1.0]pentane-1-carboxylate (16f) ${ }^{13} \mathrm{C} \mathrm{NMR}\left(\mathrm{CDCl}_{3}\right)$ 

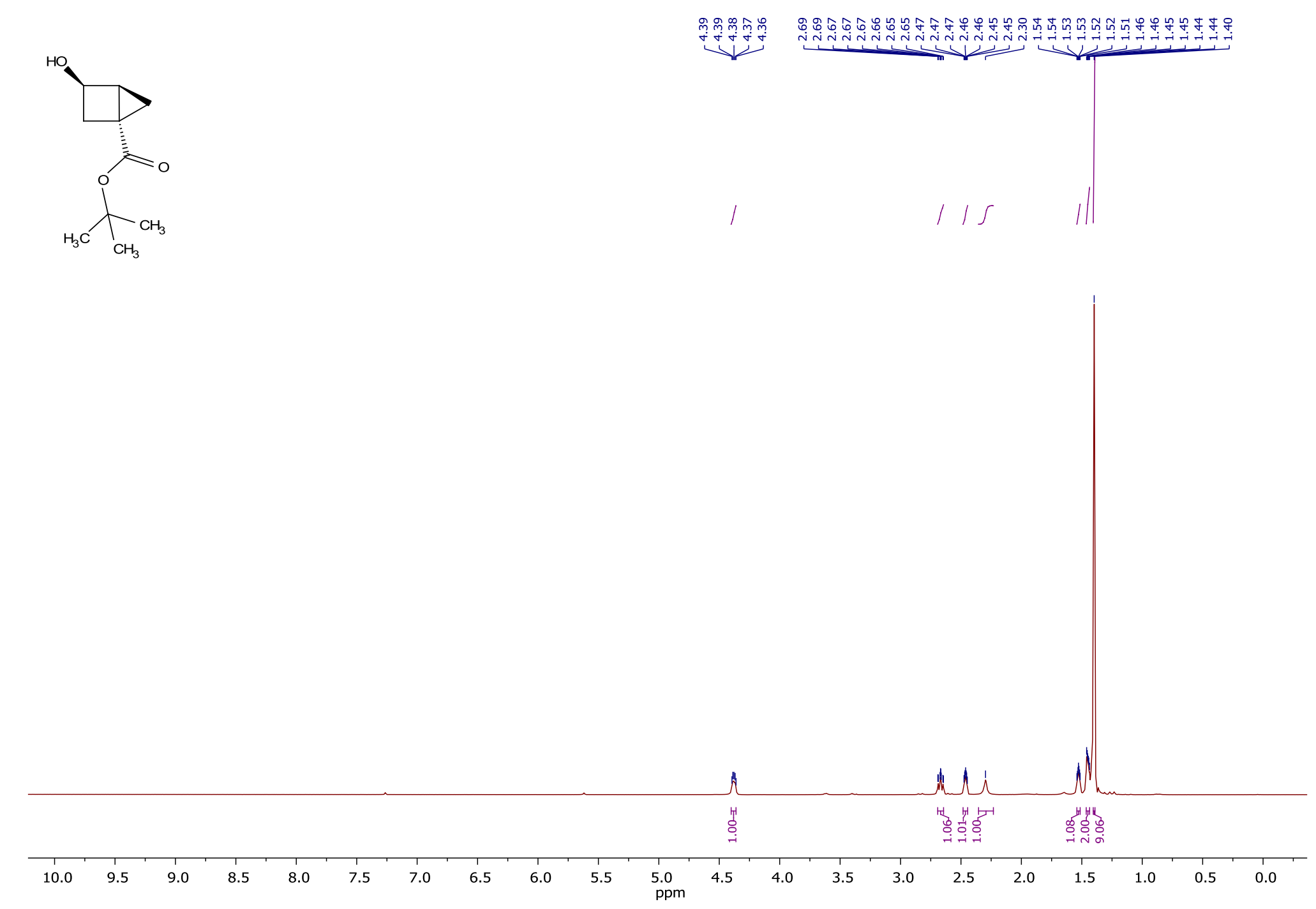

$\left(1 R^{*}, 3 R^{*}, 4 S^{*}\right)$-tert-Butyl 3-hydroxybicyclo[2.1.0]pentane-1-carboxylate (16g) ${ }^{1} \mathrm{H} \mathrm{NMR}\left(\mathrm{CDCl}_{3}\right)$ 

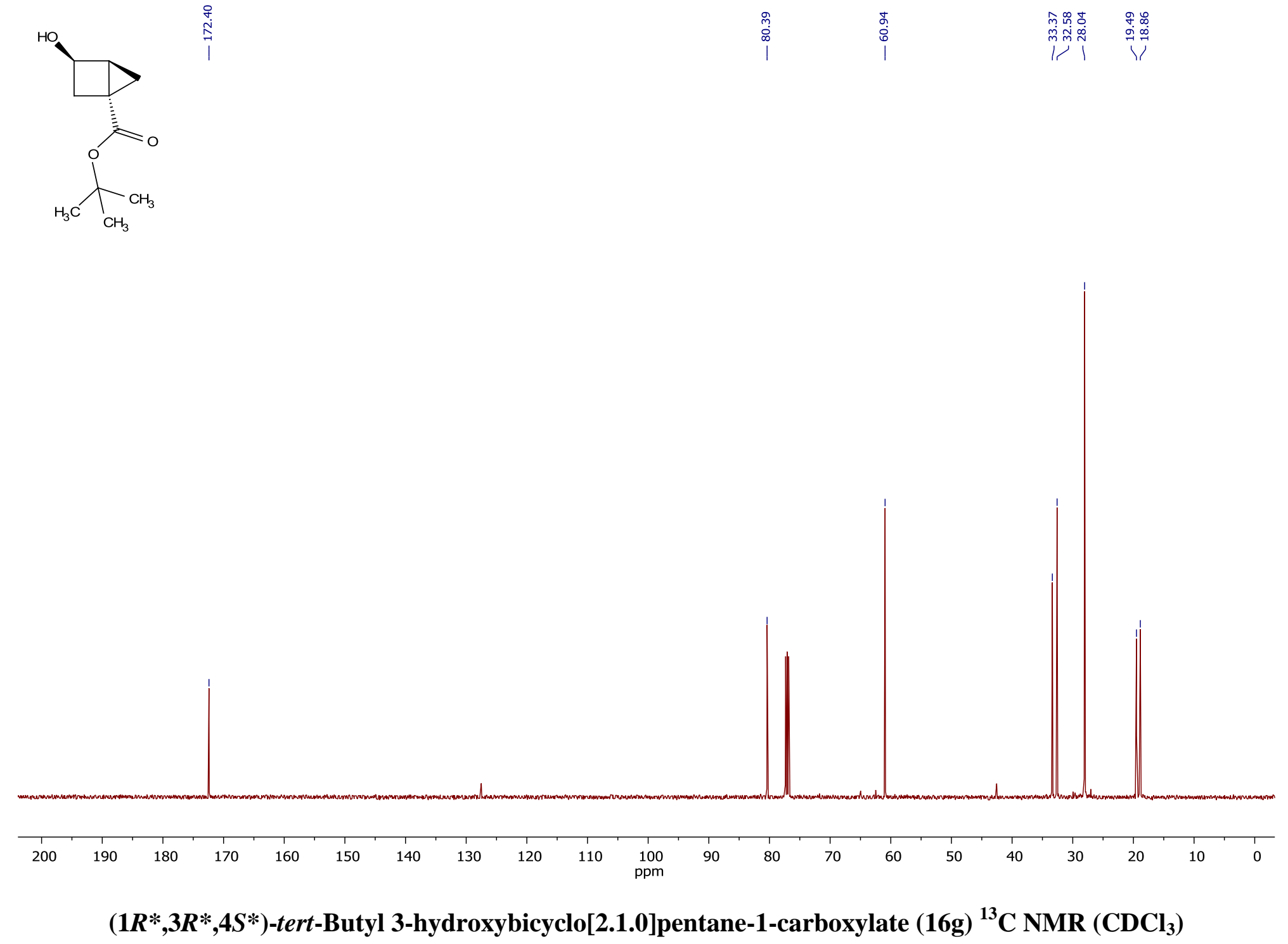


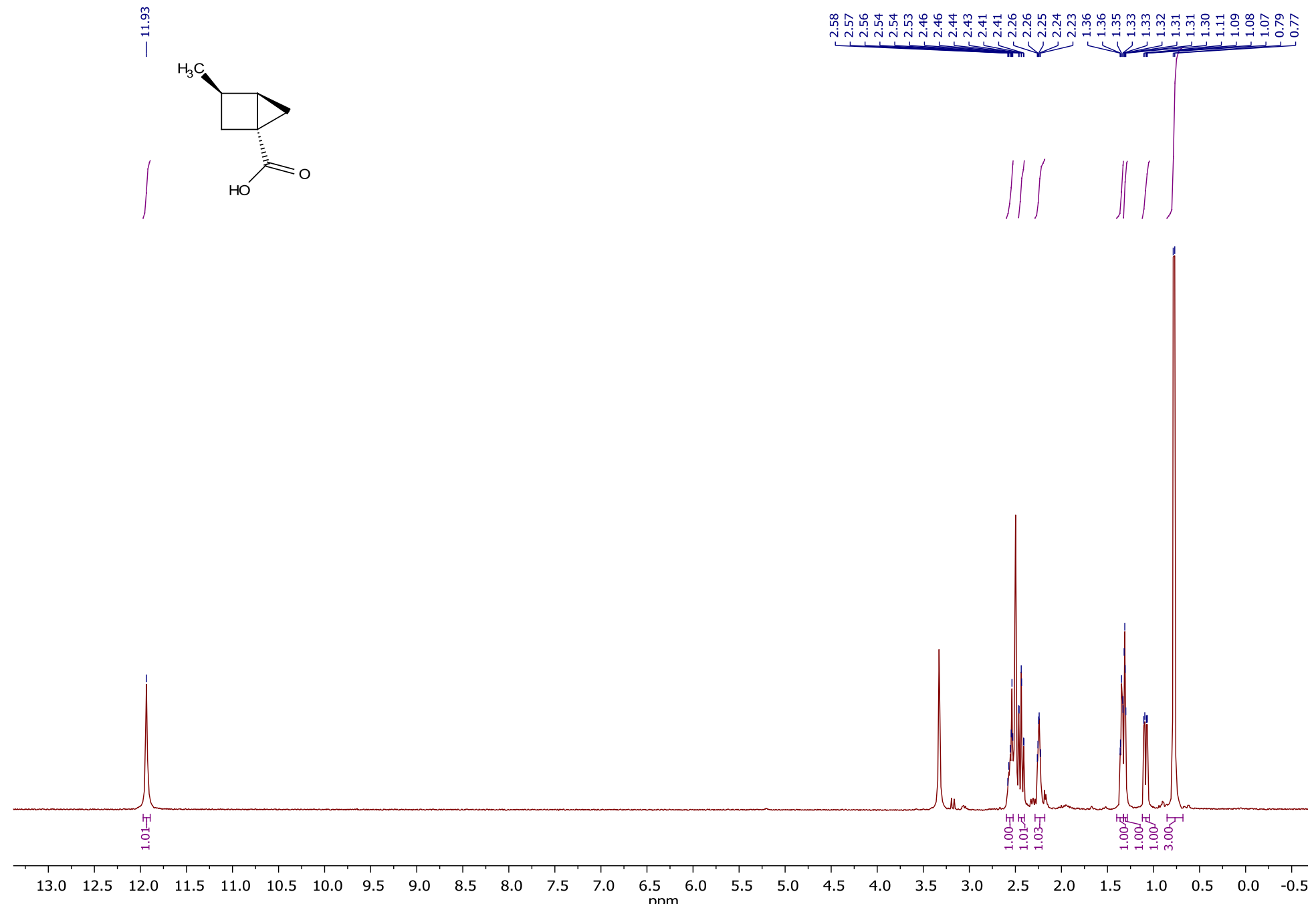

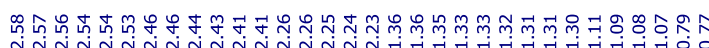

$\left(1 R^{*}, 3 R^{*}, 4 R^{*}\right)-3$-Methylbicyclo[2.1.0]pentane-1-carboxylic acid (17a) ${ }^{1} \mathrm{H}$ NMR (DMSO- $\left.d_{6}\right)$ 

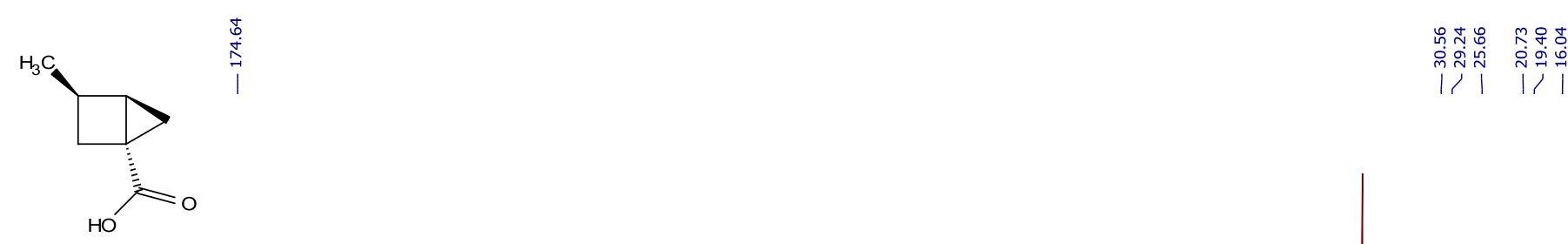

लें ก่

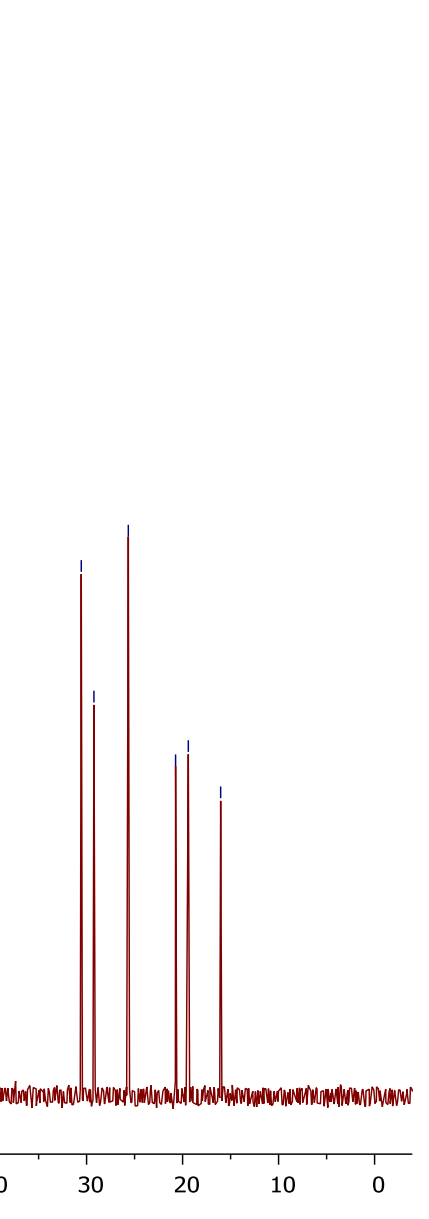

$\left(1 R^{*}, 3 R^{*}, 4 R^{*}\right)-3-M e t h y l b i c y c l o[2.1 .0]$ pentane-1-carboxylic acid (17a) ${ }^{13} \mathrm{C}$ NMR (DMSO- $\left.d_{6}\right)$ 

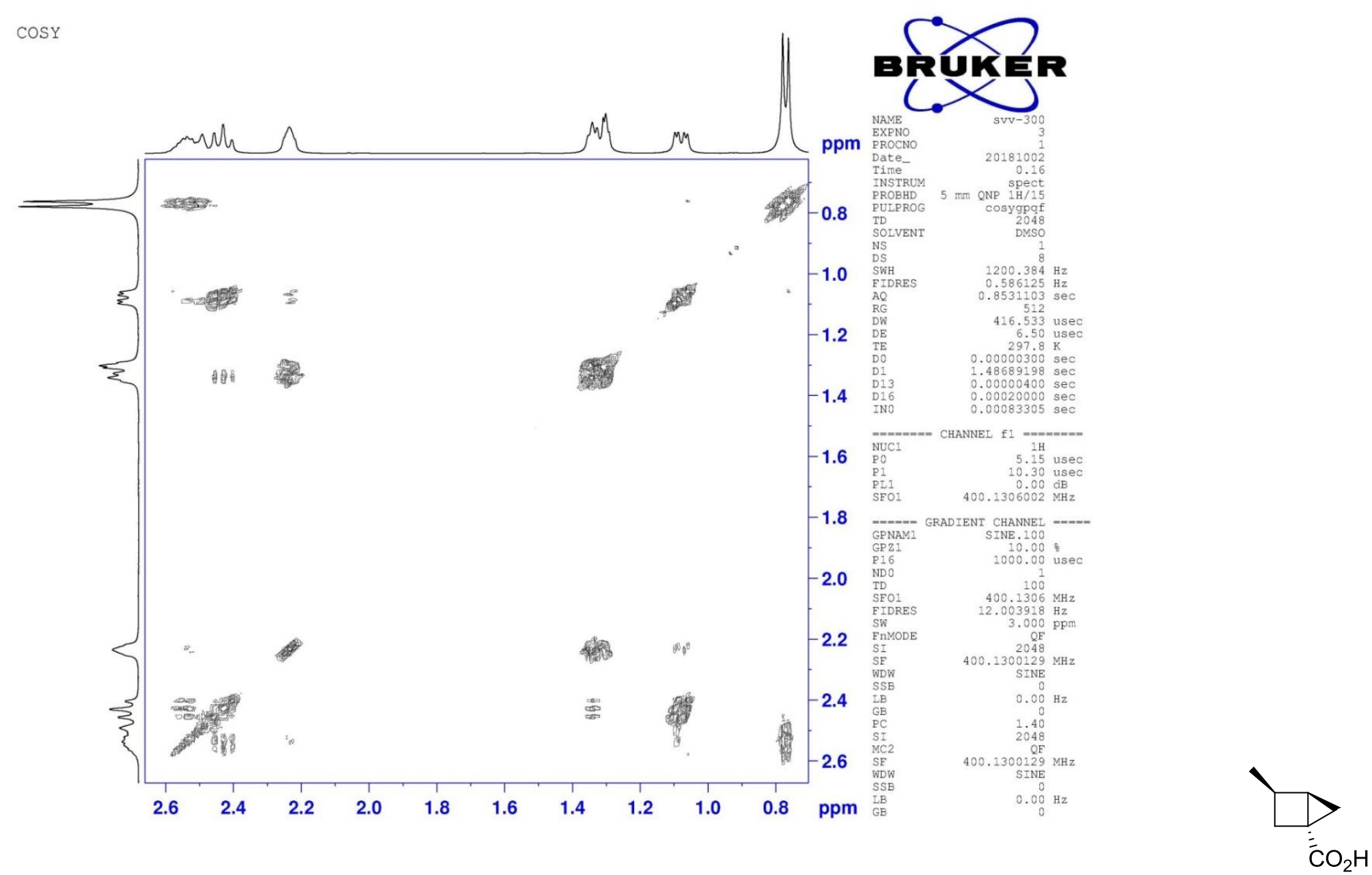

$\left(1 R^{*}, 3 R^{*}, 4 R^{*}\right)$-3-Methylbicyclo[2.1.0]pentane-1-carboxylic acid (17a) (COSY) (DMSO- $\left.d_{6}\right)$ 

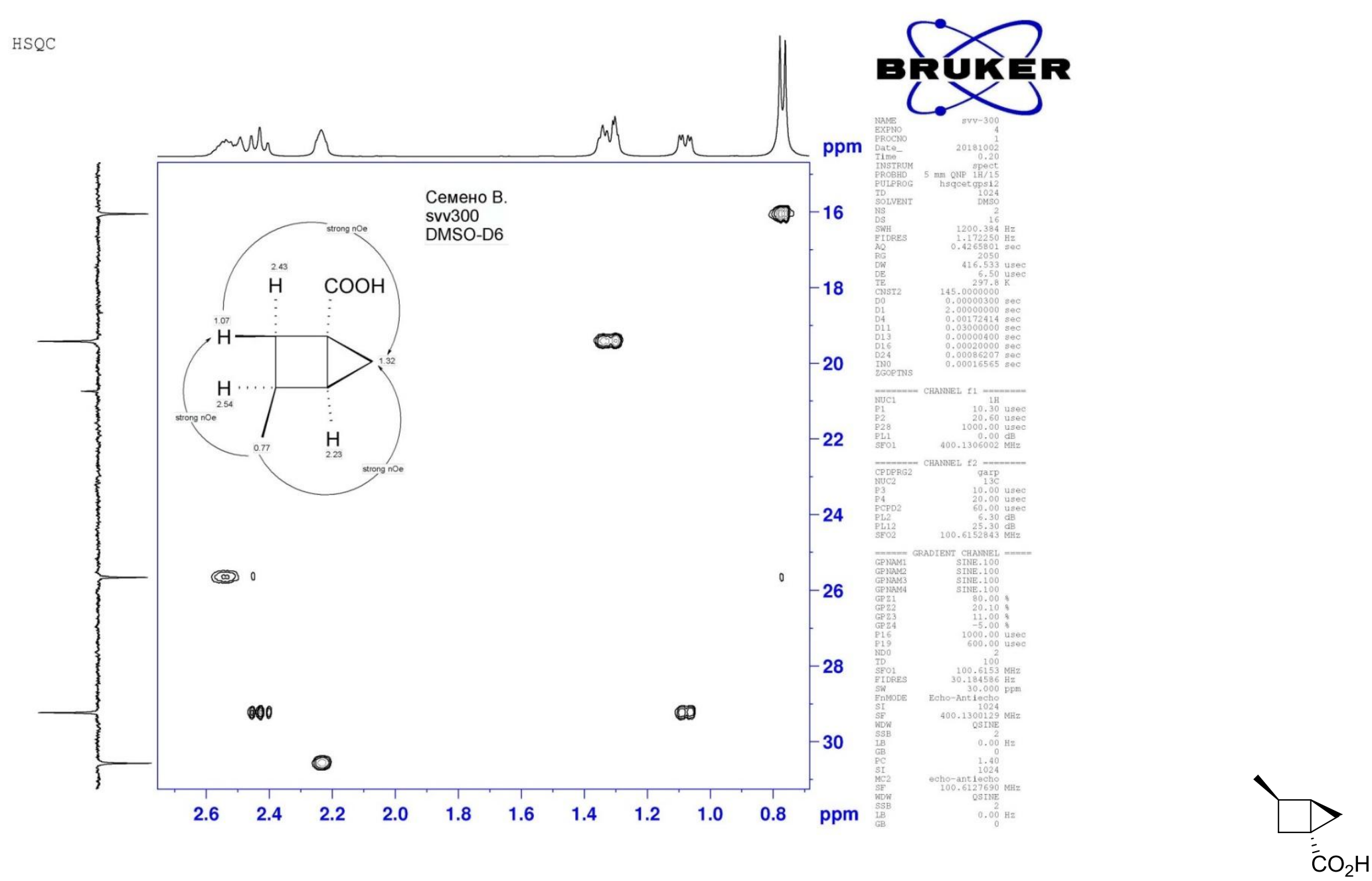

$\left(1 R^{*}, 3 R^{*}, 4 R^{*}\right)-3$-Methylbicyclo[2.1.0]pentane-1-carboxylic acid (17a) (HSQC) (DMSO-d $\left._{6}\right)$ 


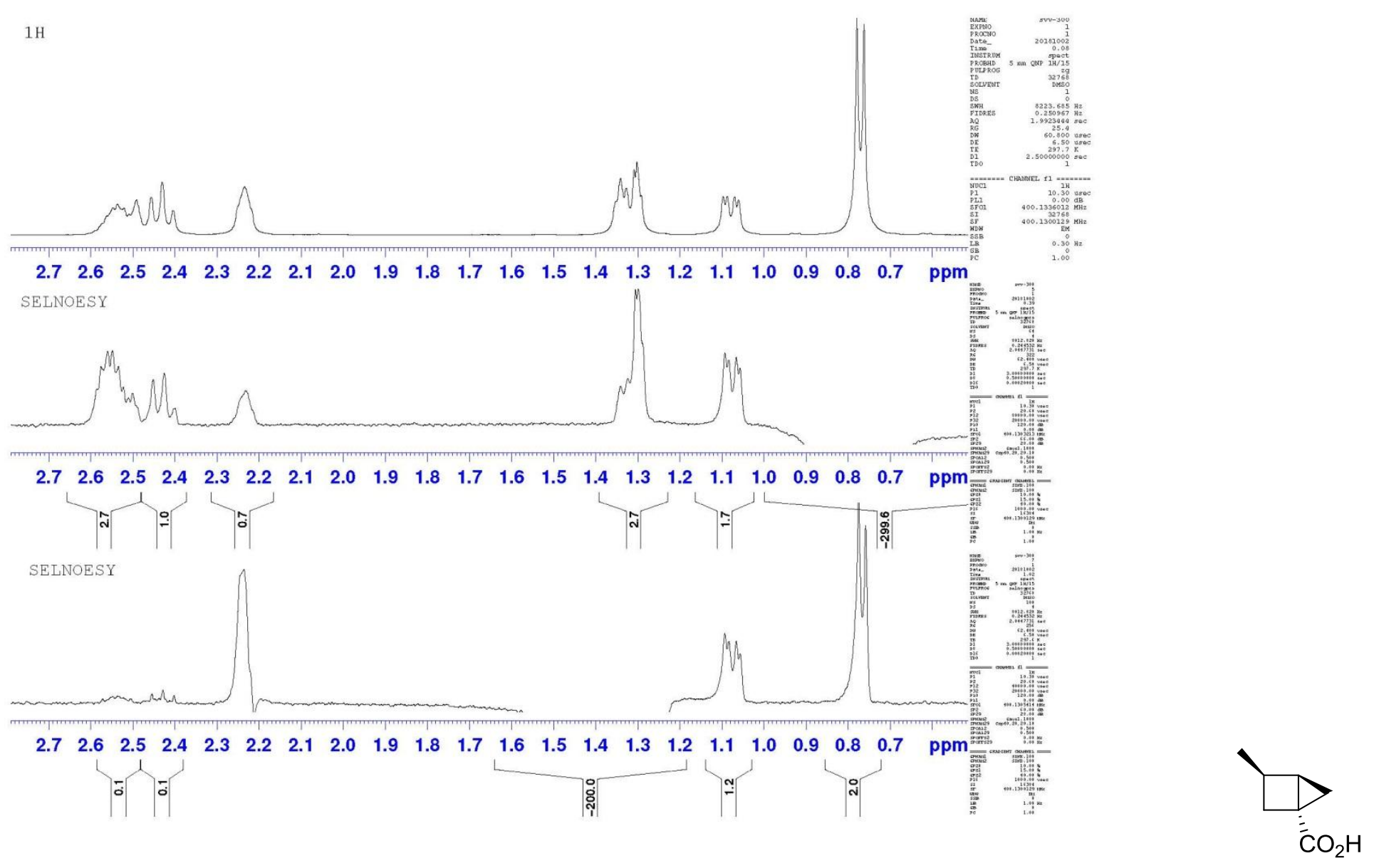

$\left(1 R^{*}, 3 R^{*}, 4 R^{*}\right)-3-$ Methylbicyclo[2.1.0]pentane-1-carboxylic acid (17a) (H-H NOE) (DMSO- $d_{6}$ ) 


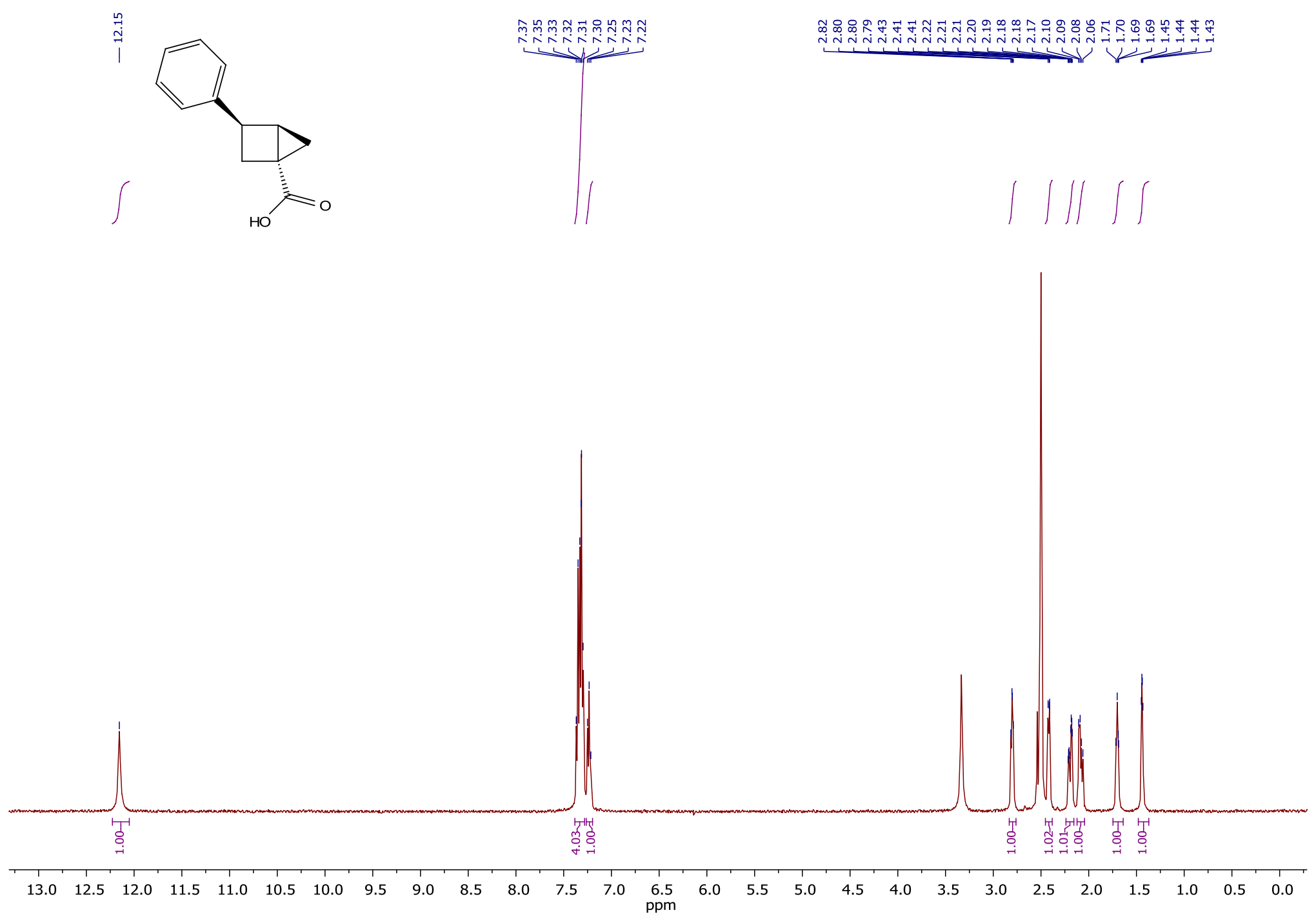

$\left(1 R^{*}, 3 R^{*}, 4 R^{*}\right)-3-P h e n y l b i c y c l o[2.1 .0]$ pentane-1-carboxylic acid (17b) ${ }^{1} \mathrm{H}$ NMR (DMSO- $\left.d_{6}\right)$ 


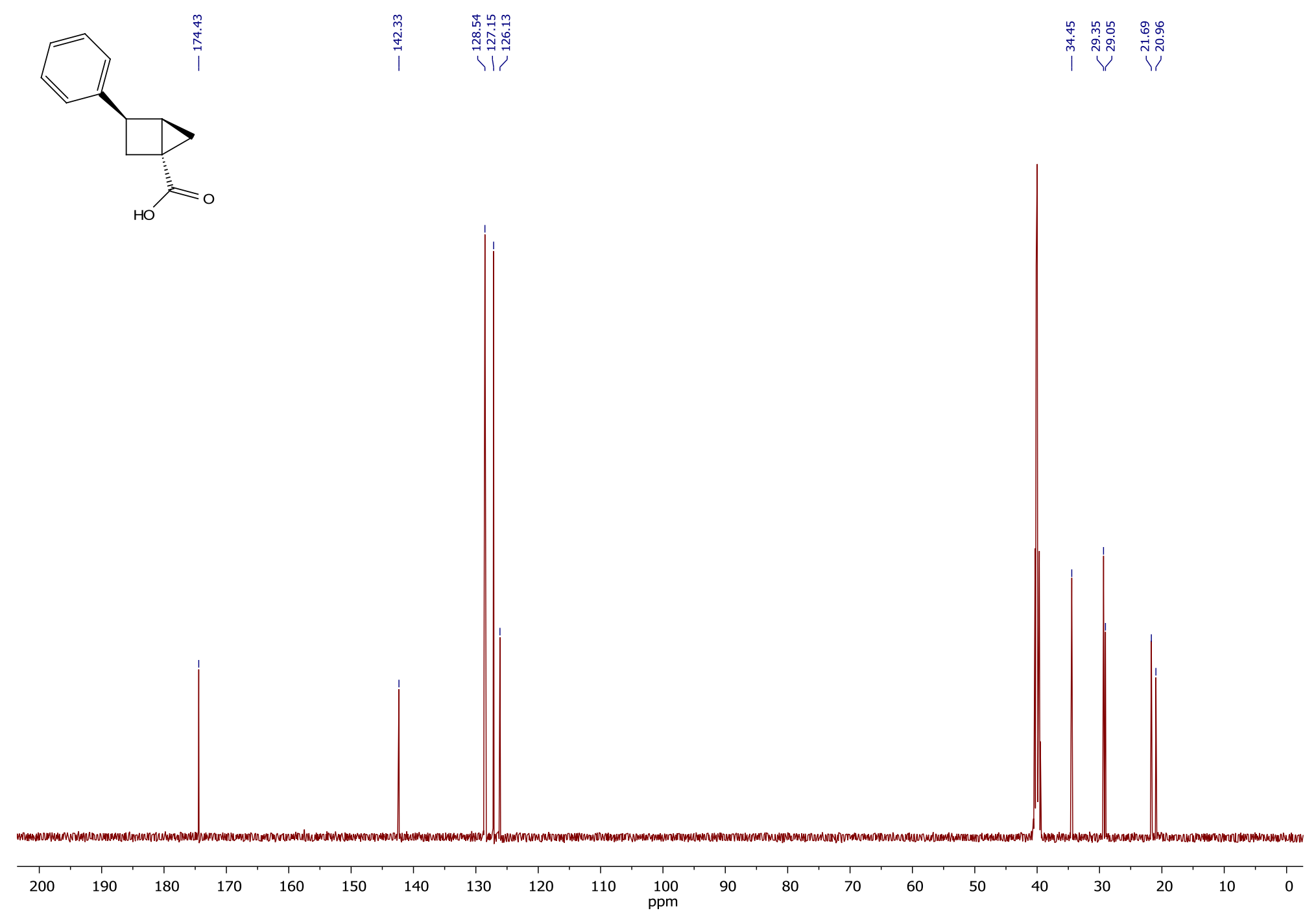

$\left(1 R^{*}, 3 R^{*}, 4 R^{*}\right)-3-$ Phenylbicyclo[2.1.0]pentane-1-carboxylic acid (17b) ${ }^{13} \mathrm{C}$ NMR (DMSO-d $)$ 

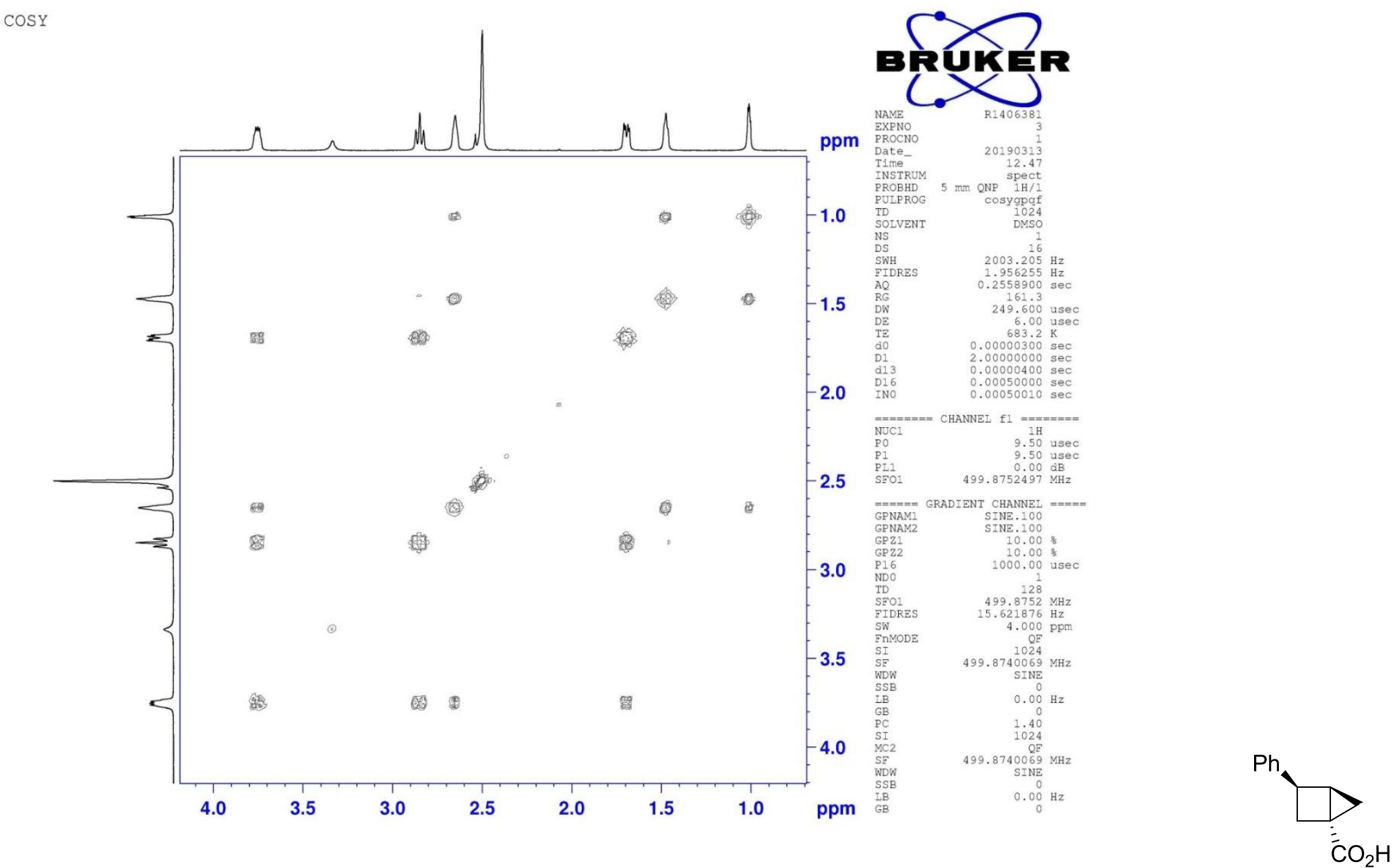

$\left(1 R^{*}, 3 R^{*}, 4 R^{*}\right)-3$-Phenylbicyclo[2.1.0]pentane-1-carboxylic acid (17b) (COSY) (DMSO-d $)$ 

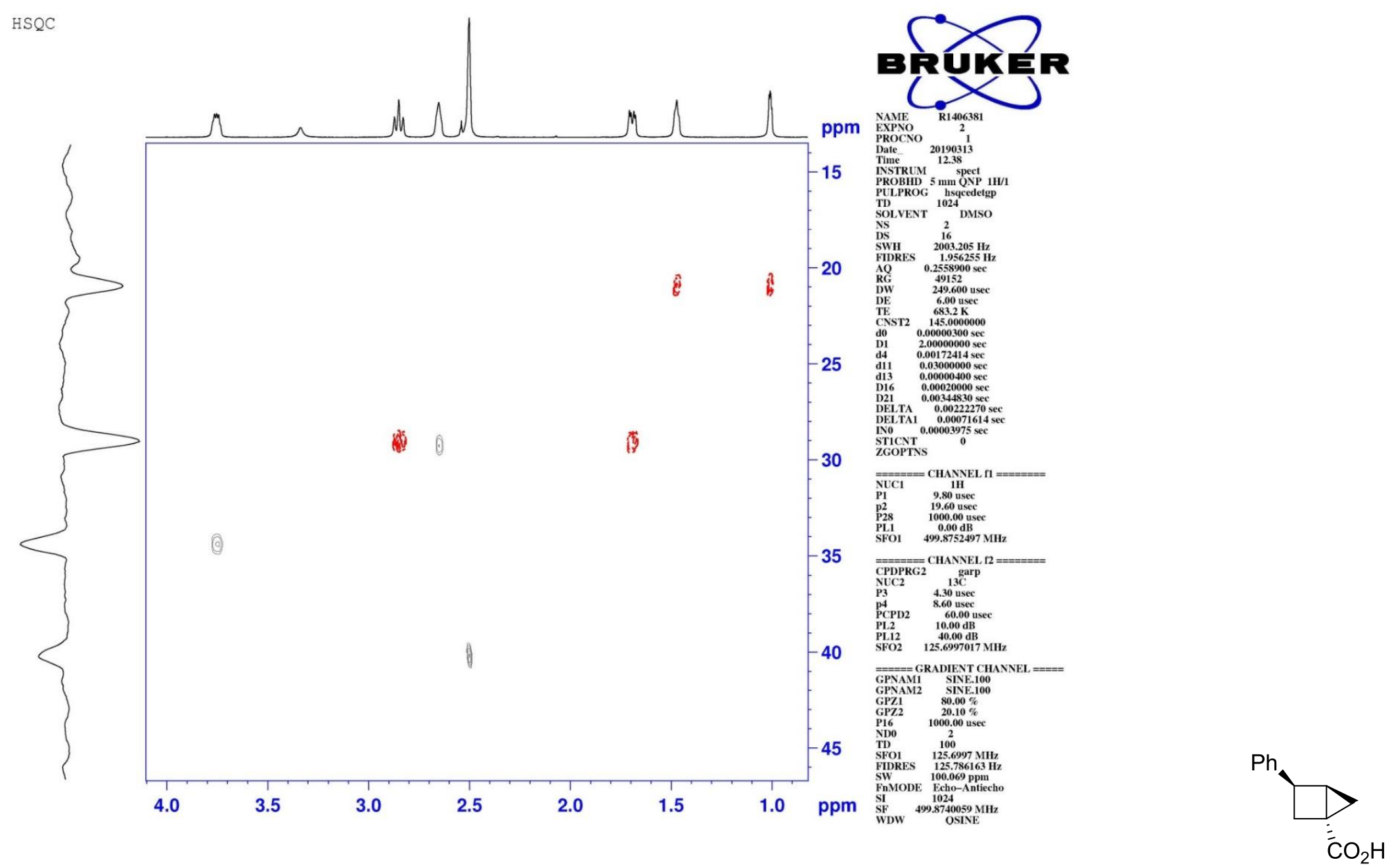

$\left(1 R^{*}, 3 R^{*}, 4 R^{*}\right)$-3-Phenylbicyclo[2.1.0]pentane-1-carboxylic acid (17b) (HSQC) (DMSO- $d_{6}$ ) 

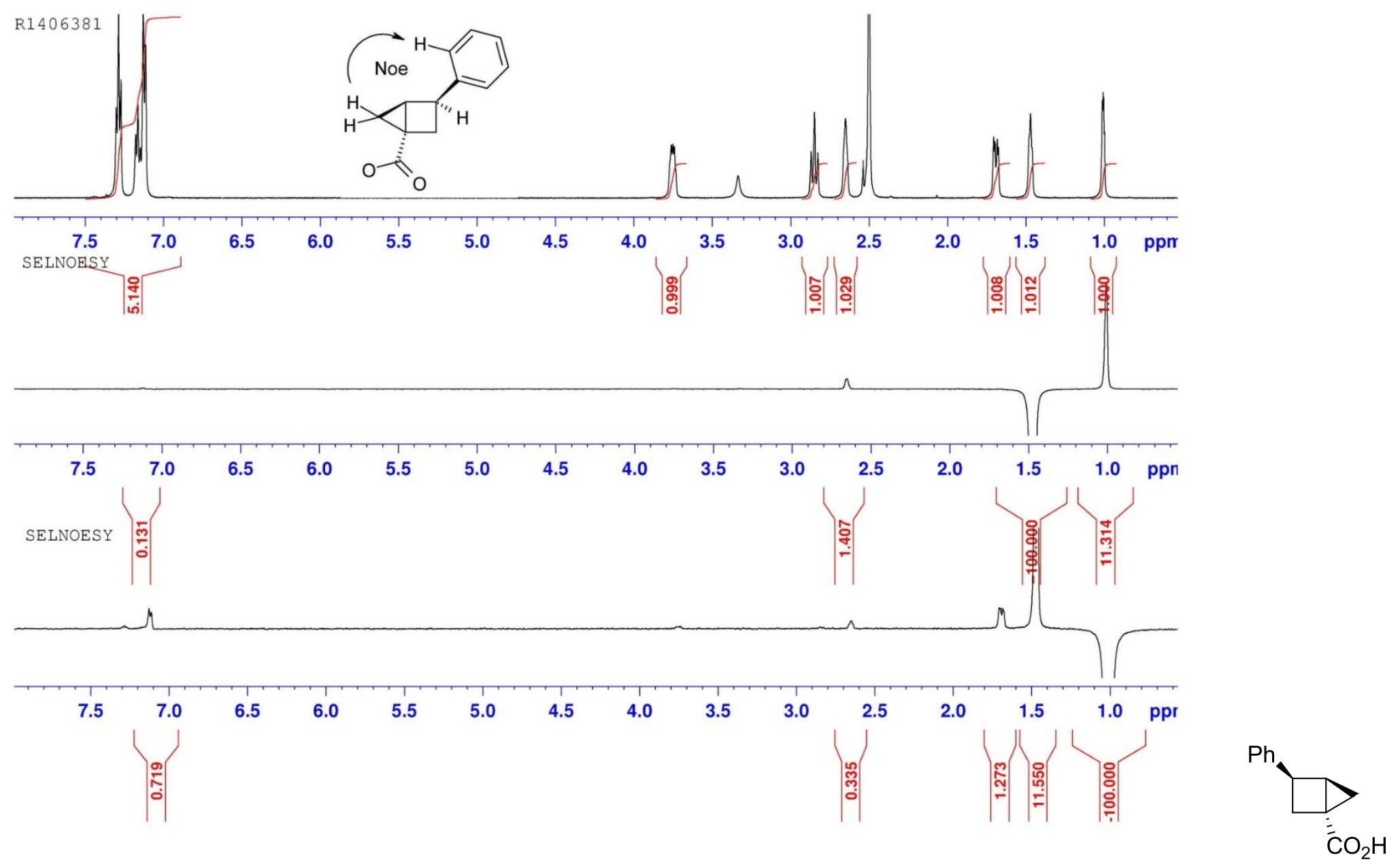

$\left(1 R^{*}, 3 R^{*}, 4 R^{*}\right)-3-P h e n y l b i c y c l o[2.1 .0]$ pentane-1-carboxylic acid (17b) (H-H NOE) (DMSO-d $)$ 


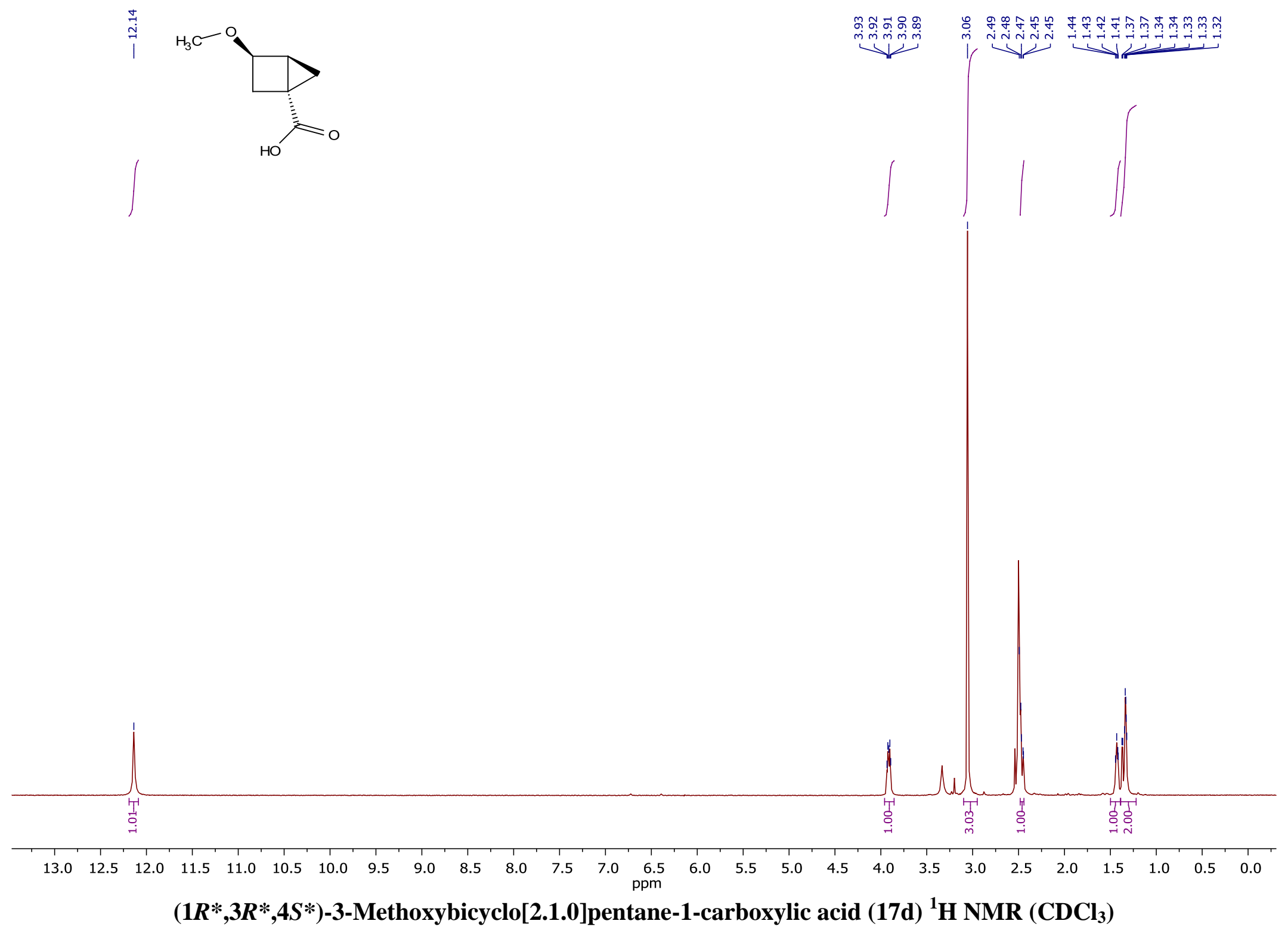




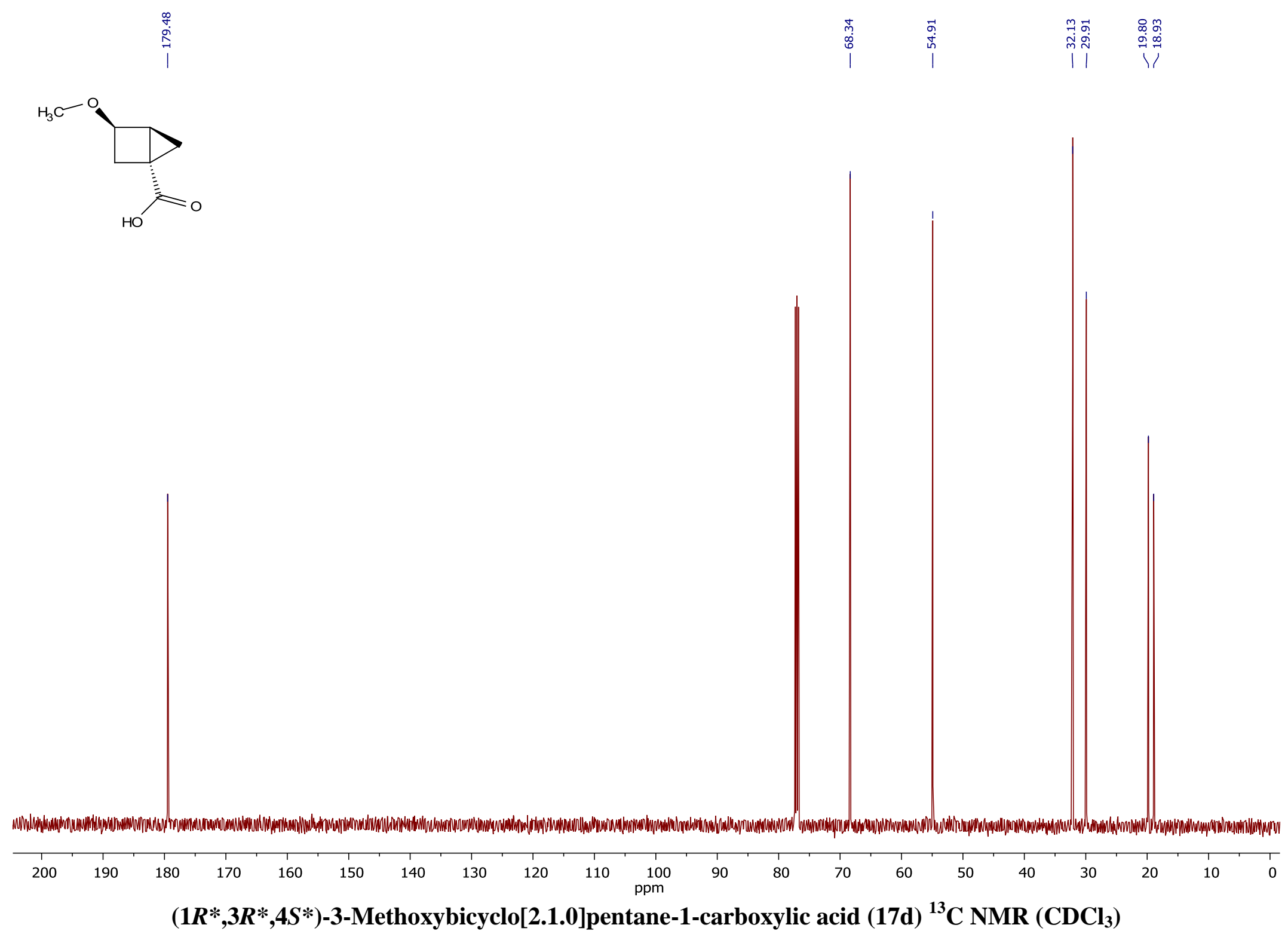




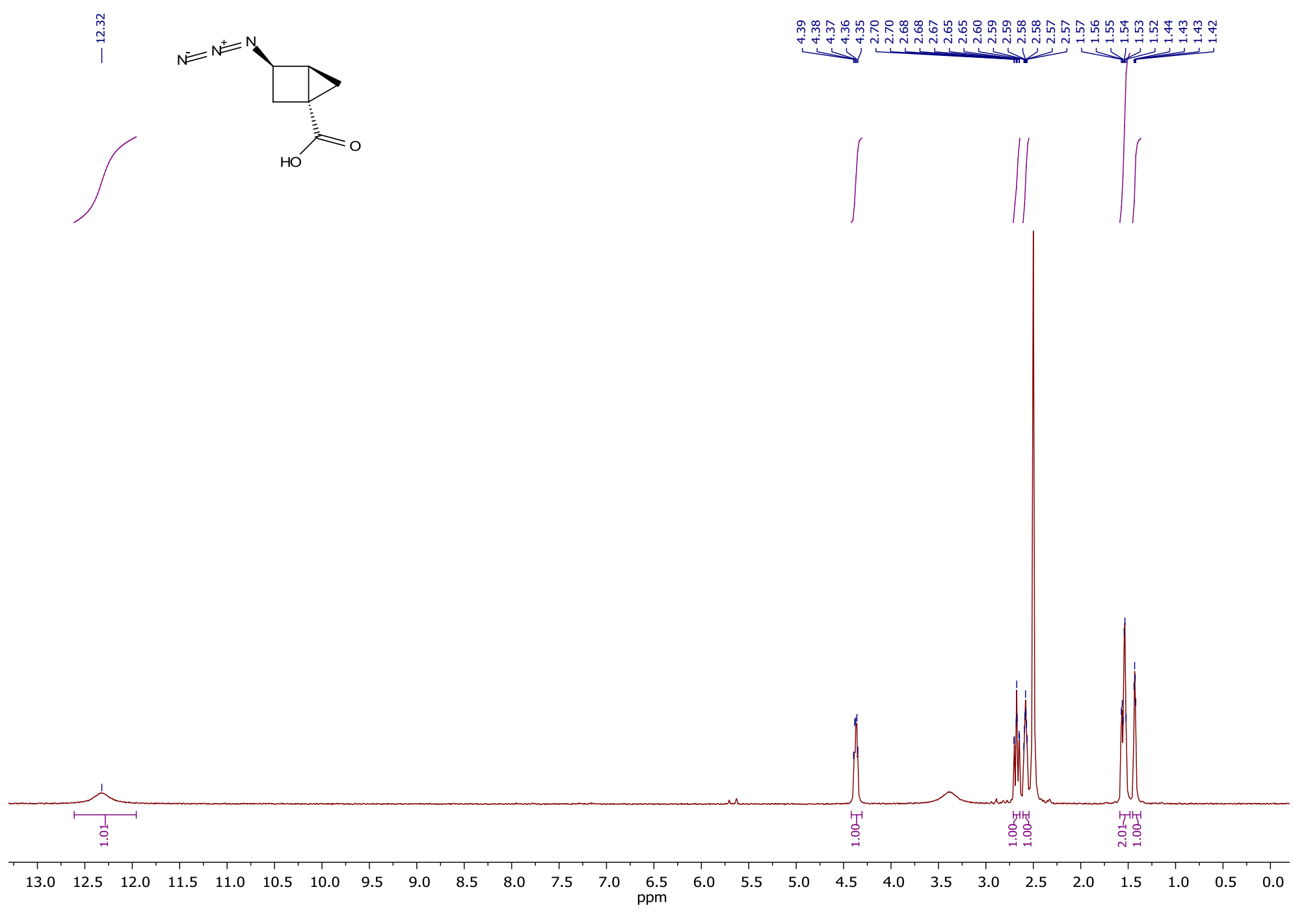

$\left(1 R^{*}, 3 R^{*}, 4 S^{*}\right)$-3-Azidobicyclo[2.1.0]pentane-1-carboxylic acid (17f) ${ }^{1}$ H NMR (DMSO-d $\left.d_{6}\right)$ 

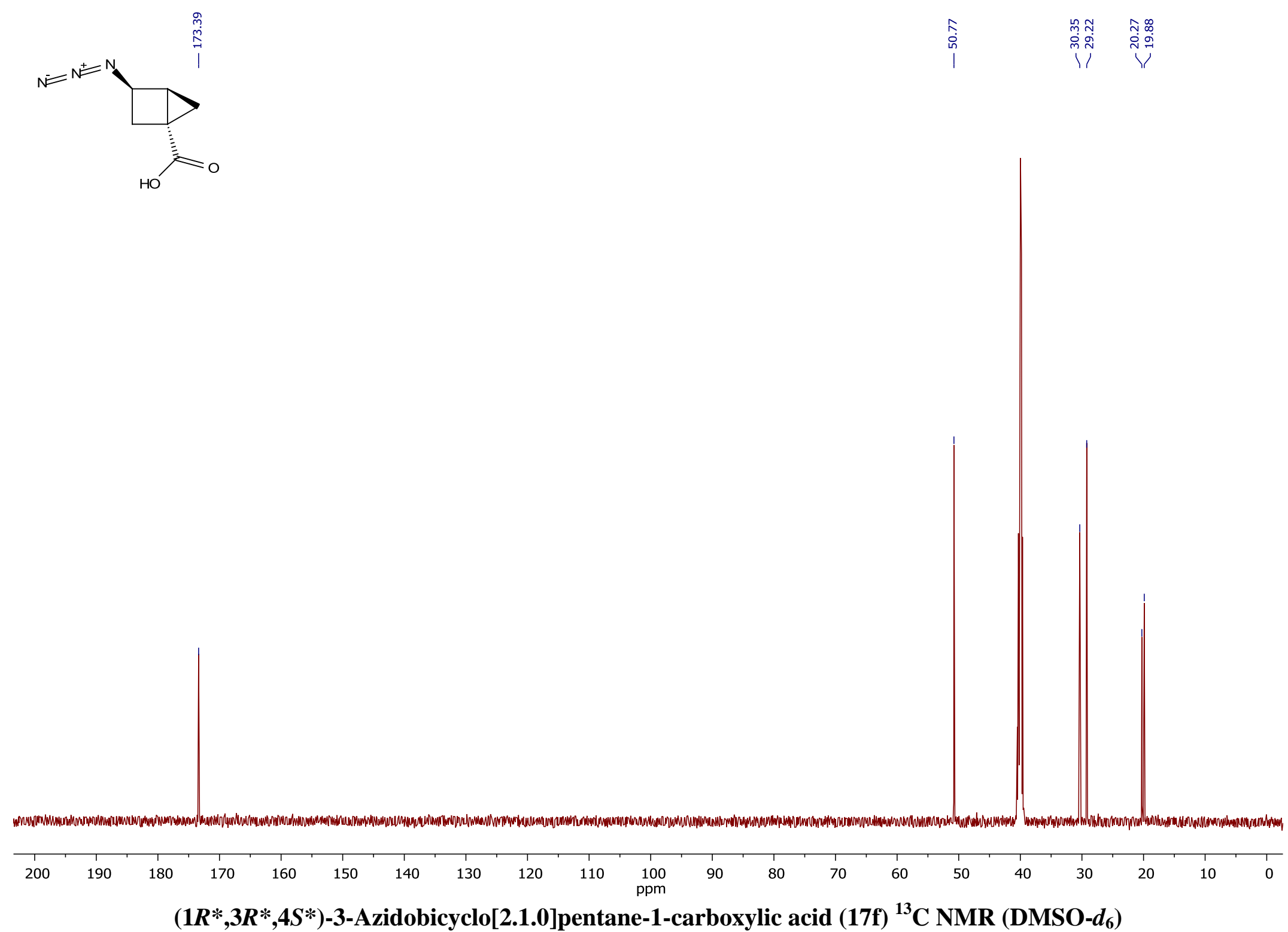


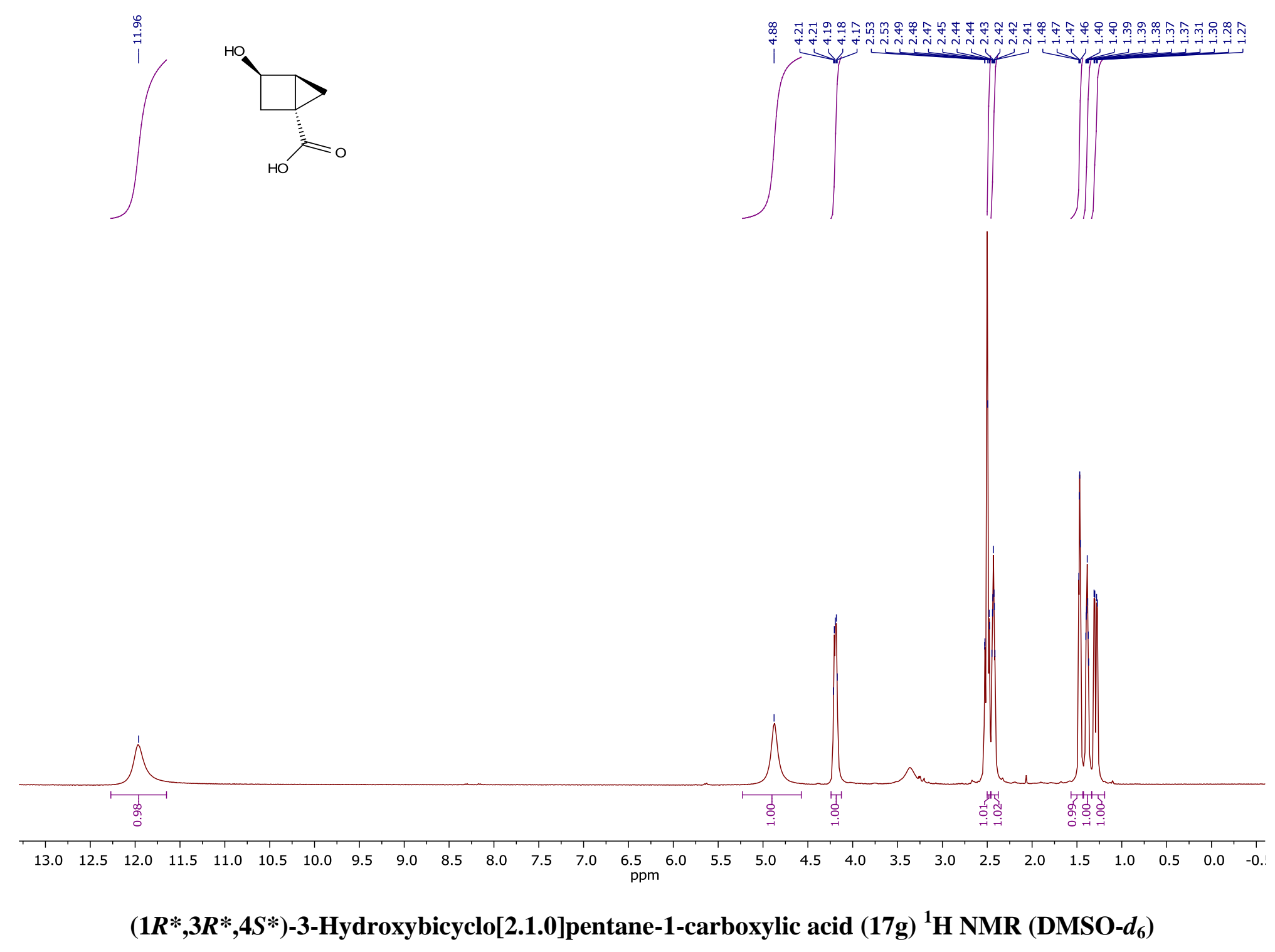




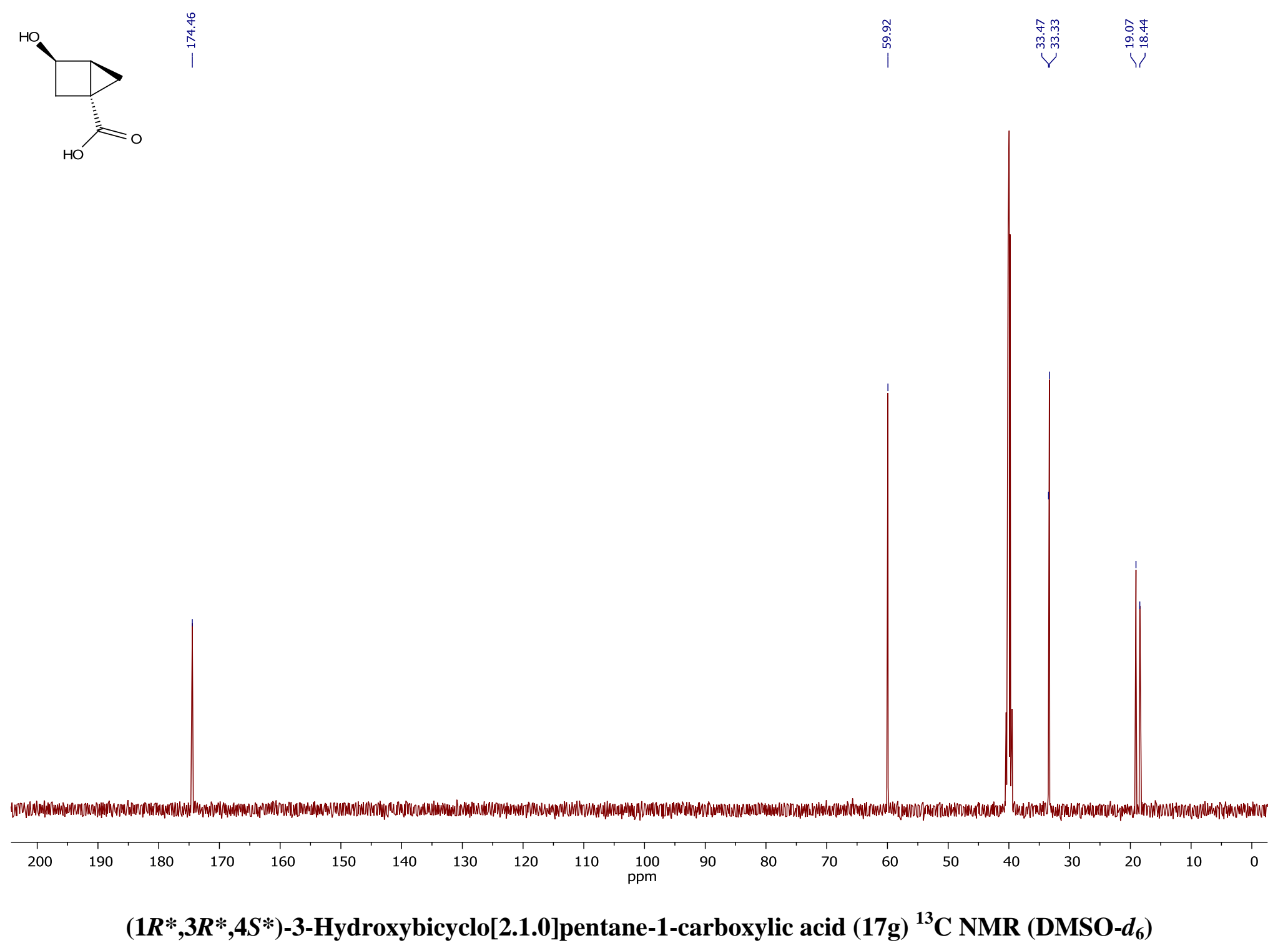




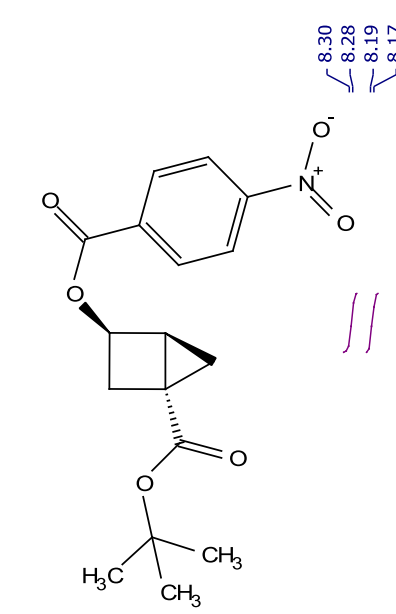

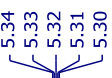

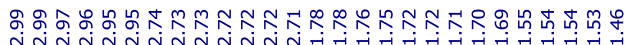
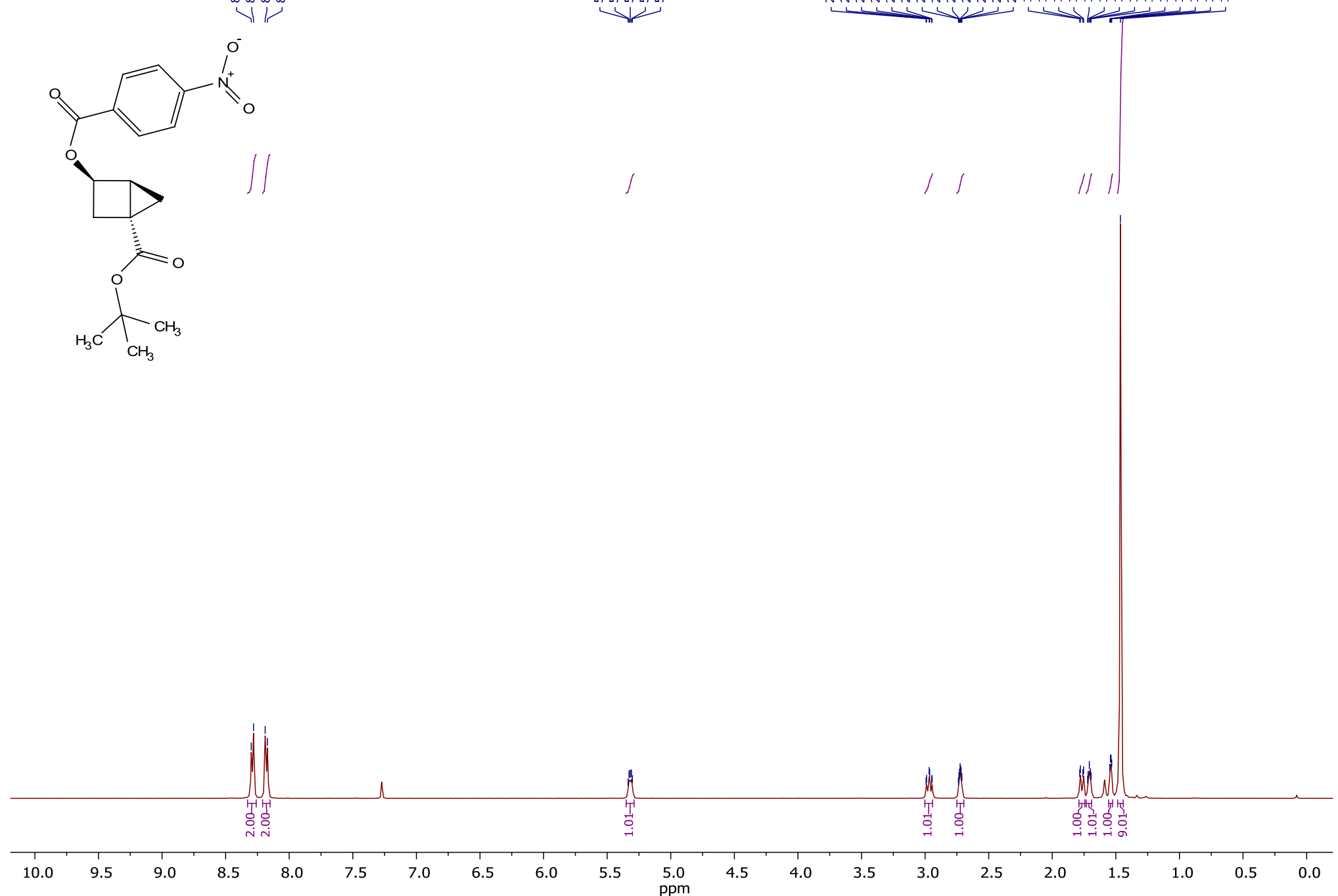

$\left(1 R^{*}, 3 R^{*}, 4 S^{*}\right)$-tert-Butyl 3-((4-nitrobenzoyl)oxy)bicyclo[2.1.0]pentane-1-carboxylate $(18 \mathrm{~g}){ }^{1} \mathrm{H}$ NMR $\left(\mathrm{CDCl}_{3}\right)$ 


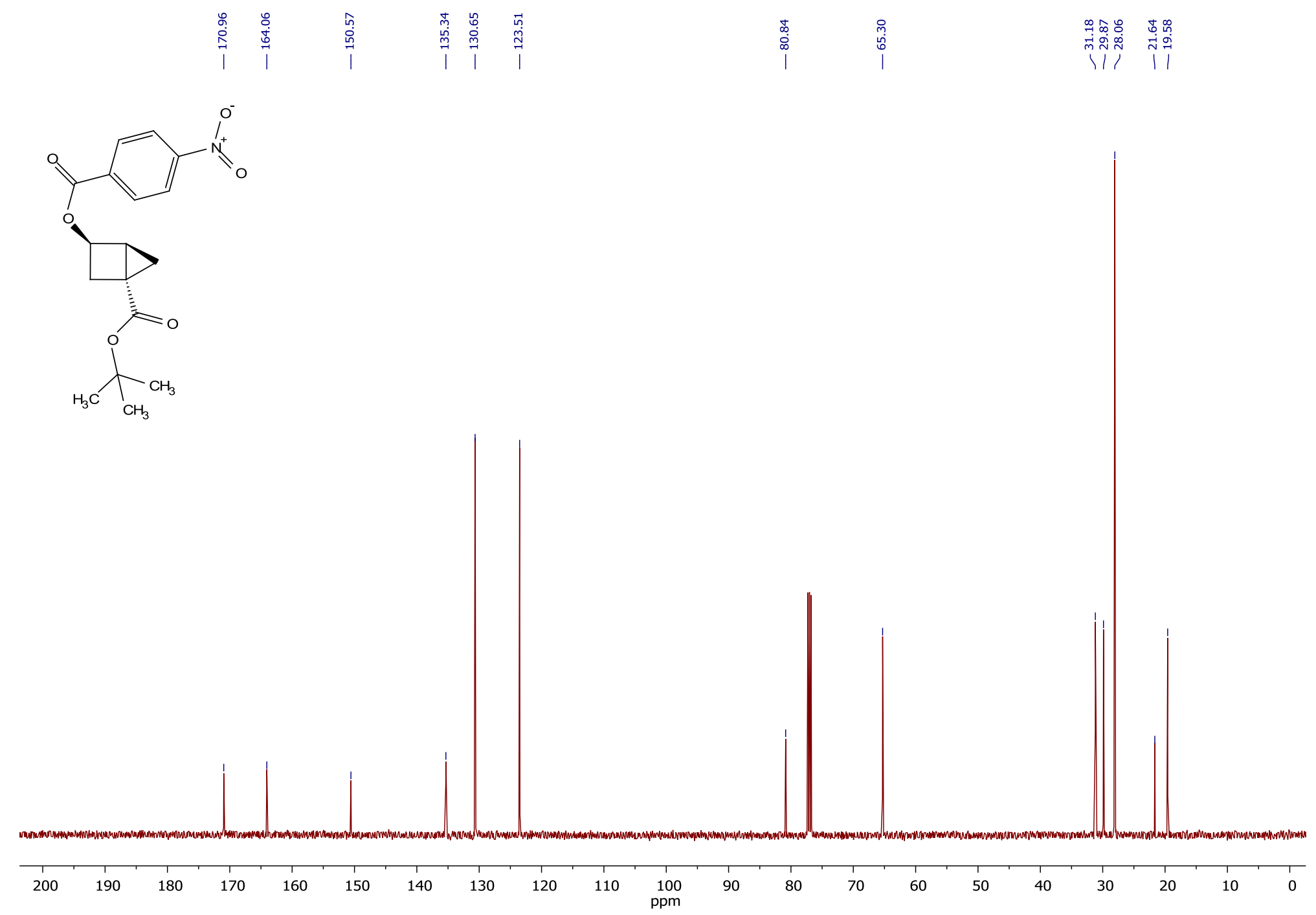

$\left(1 R^{*}, 3 R^{*}, 4 S^{*}\right)$-tert-Butyl 3-((4-nitrobenzoyl)oxy)bicyclo[2.1.0]pentane-1-carboxylate $(18 \mathrm{~g}){ }^{13} \mathrm{C} \mathrm{NMR}\left(\mathrm{CDCl}_{3}\right)$ 

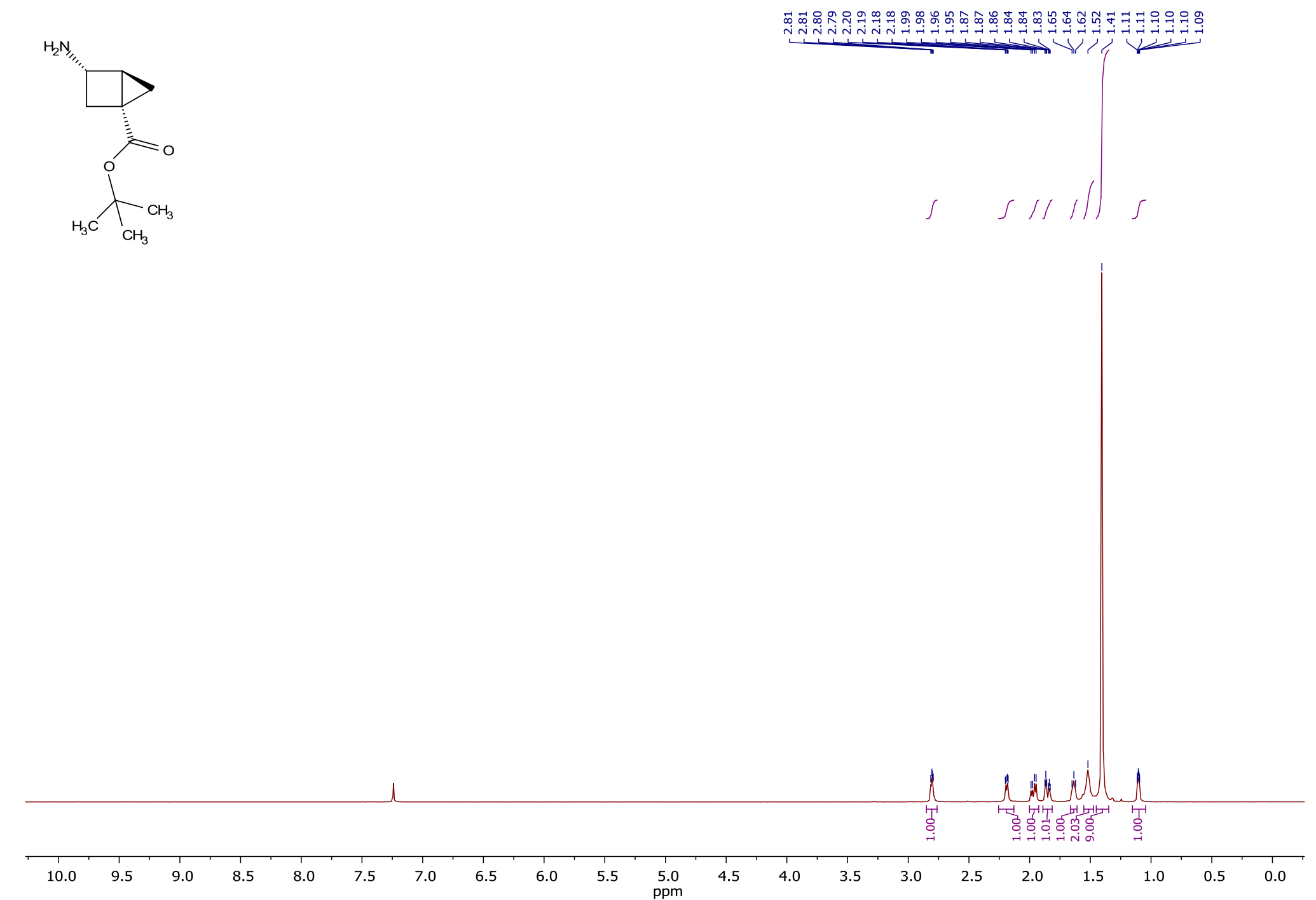

$\left(1 R^{*}, 3 S^{*}, 4 S^{*}\right)$-tert-Butyl 3-aminobicyclo[2.1.0]pentane-1-carboxylate (19) ${ }^{1} \mathrm{H}$ NMR $\left(\mathrm{CDCl}_{3}\right)$ 


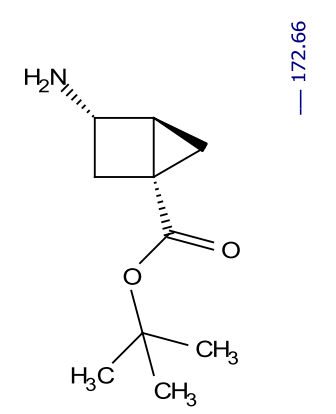

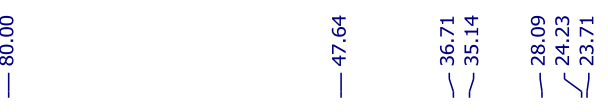

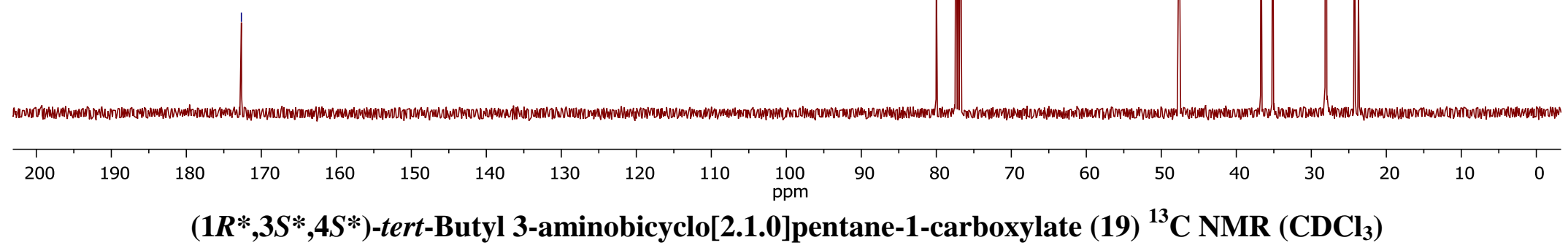



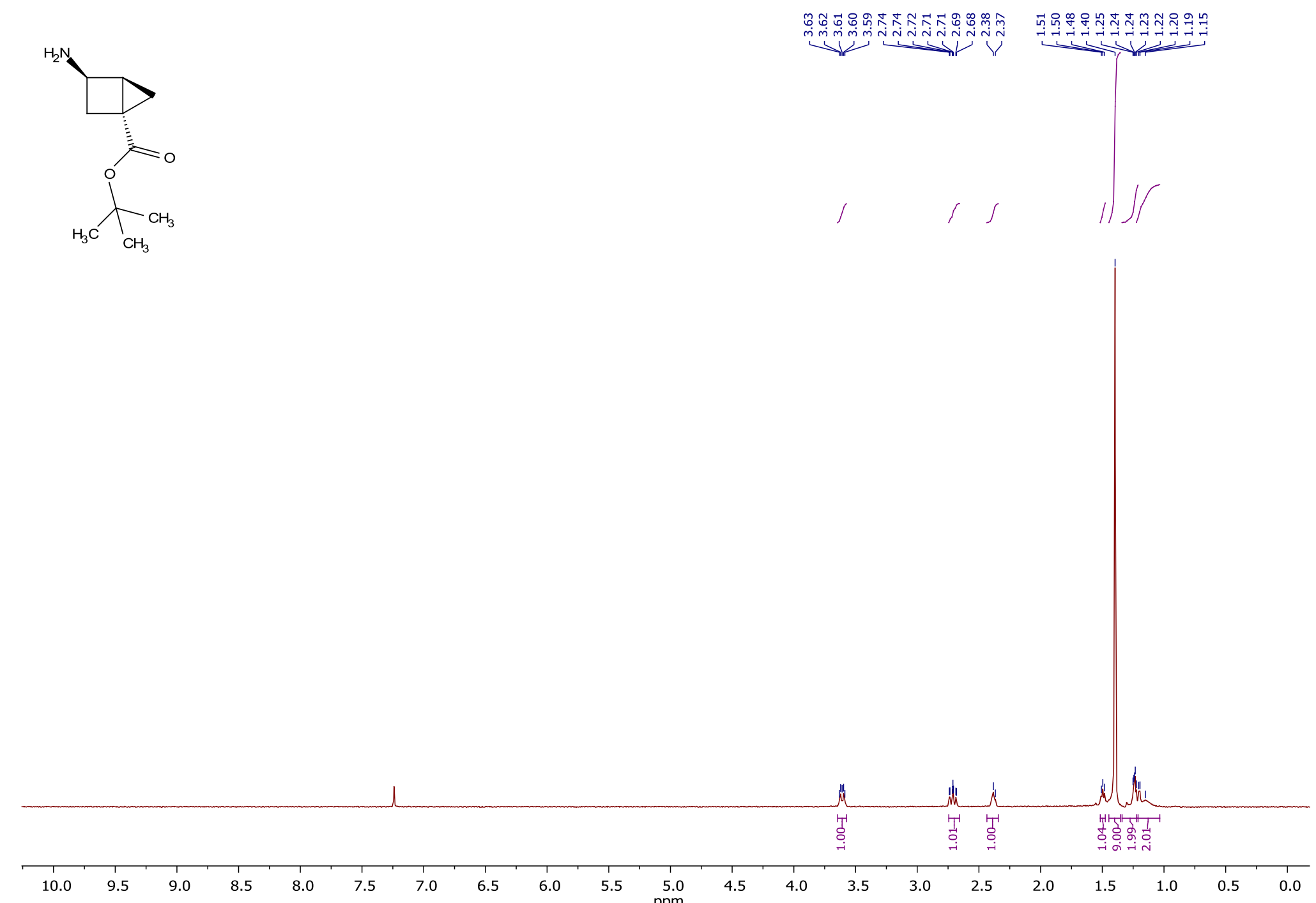

$\left(1 R^{*}, 3 R^{*}, 4 S^{*}\right)$-tert-Butyl 3-aminobicyclo[2.1.0]pentane-1-carboxylate (20) ${ }^{1} \mathrm{H}$ NMR $\left(\mathrm{CDCl}_{3}\right)$ 

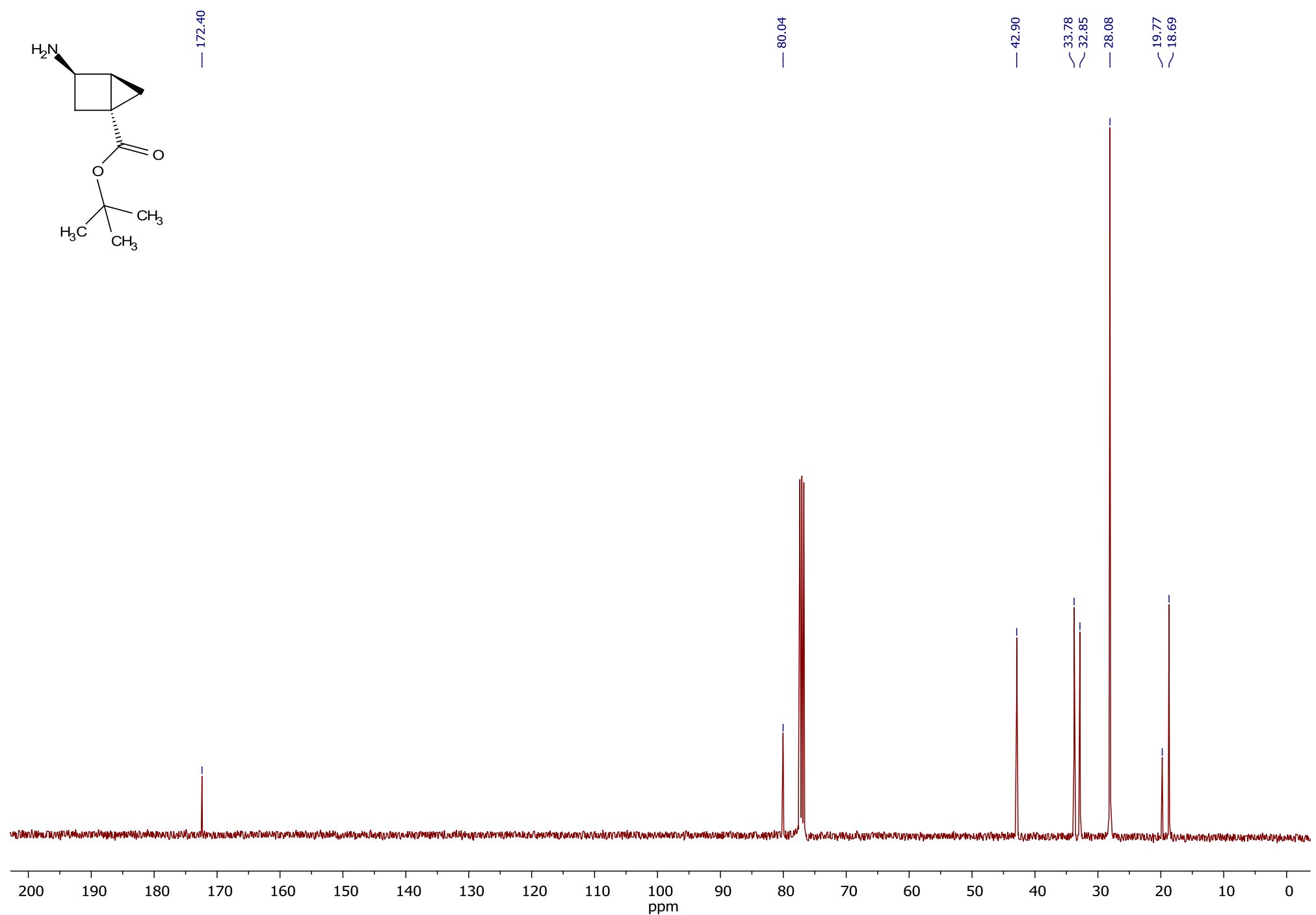

$\left(1 R^{*}, 3 R^{*}, 4 S^{*}\right)$-tert-Butyl 3-aminobicyclo[2.1.0]pentane-1-carboxylate (20) ${ }^{1} \mathrm{H}$ NMR $\left(\mathrm{CDCl}_{3}\right)$ 


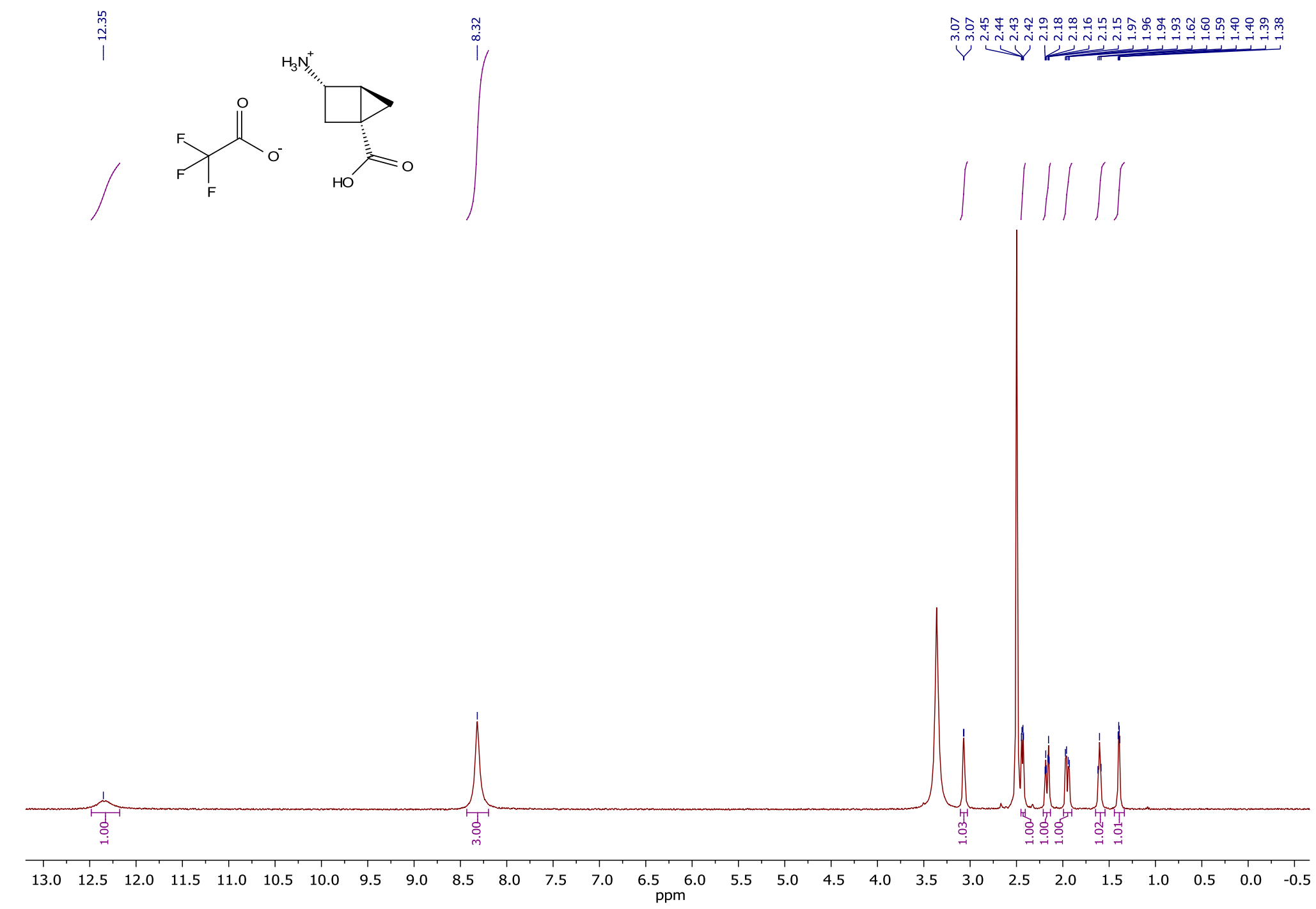

$\left(1 S^{*}, 2 S^{*}, 4 R^{*}\right)$-4-Carboxybicyclo[2.1.0]pentan-2-aminium 2,2,2-trifluoroacetate (21) ${ }^{1} \mathrm{H}$ NMR (DMSO-d $\left.d_{6}\right)$ 

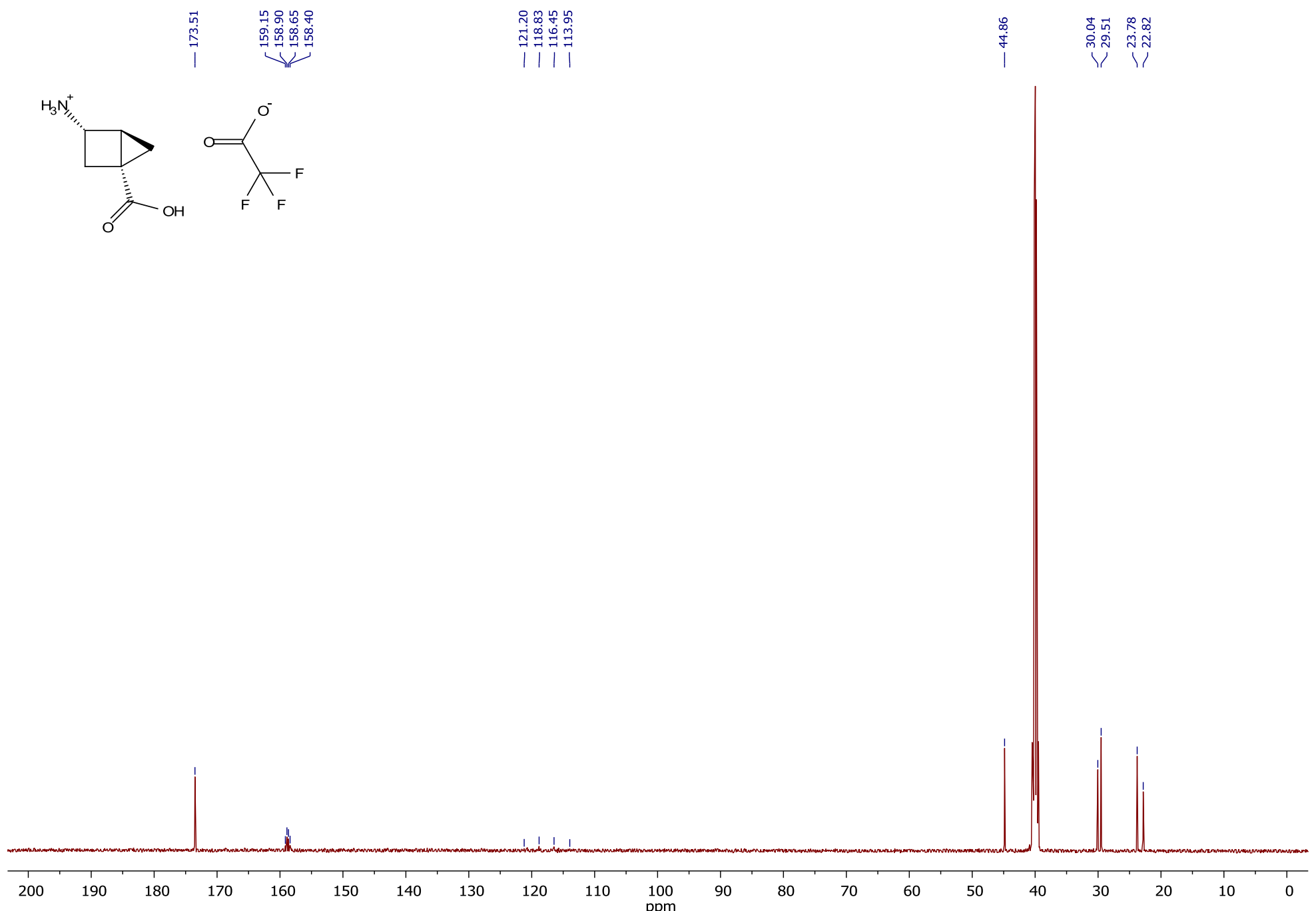

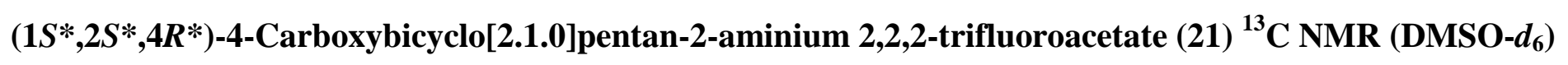



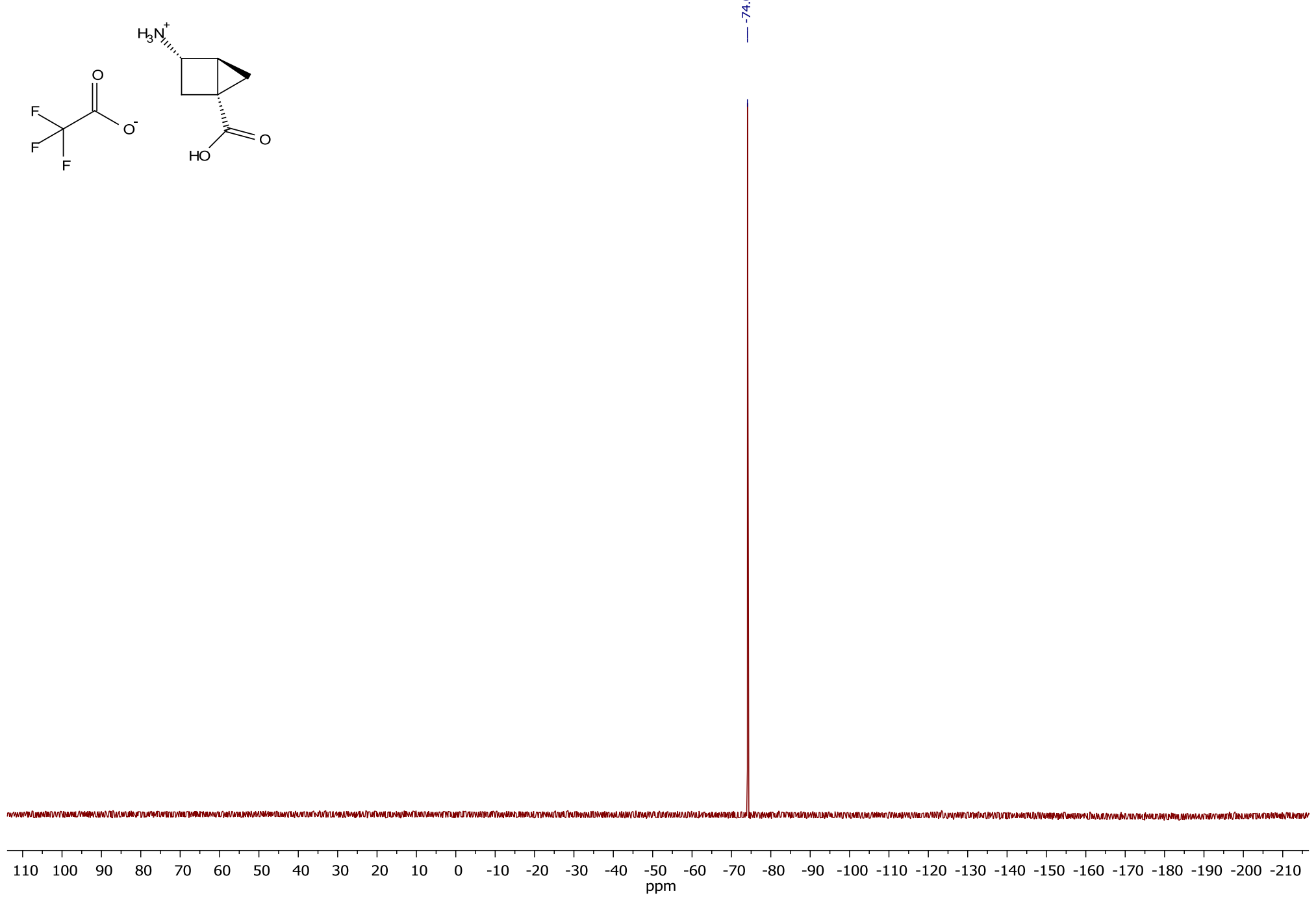

$\left(1 S^{*}, 2 S^{*}, 4 R^{*}\right)$-4-Carboxybicyclo[2.1.0]pentan-2-aminium 2,2,2-trifluoroacetate (21) ${ }^{19} \mathrm{~F}$ NMR (DMSO- $d_{6}$ ) 

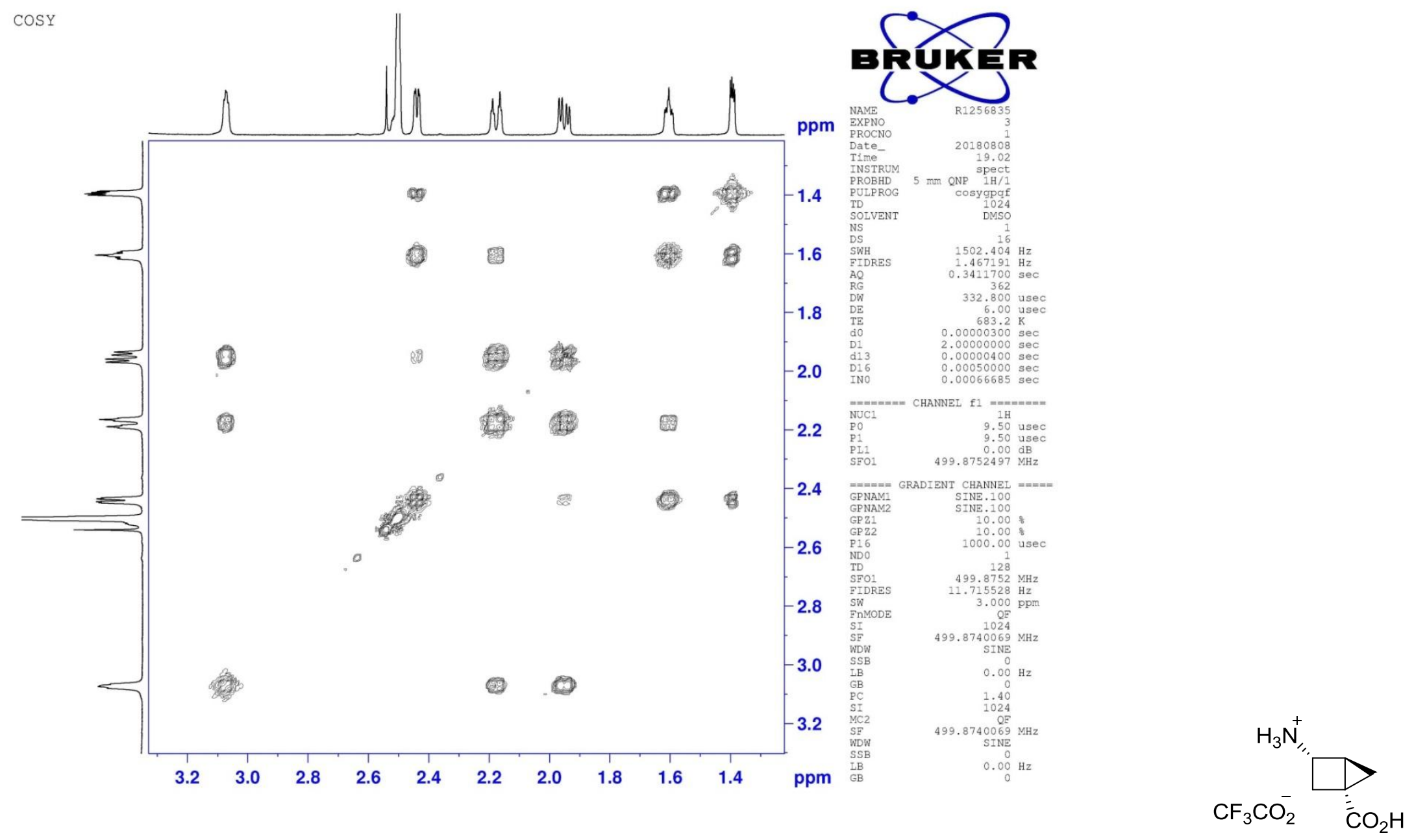

$\left(1 S^{*}, 2 S^{*}, 4 R^{*}\right)$-4-Carboxybicyclo[2.1.0]pentan-2-aminium 2,2,2-trifluoroacetate (21) (COSY) (DMSO- $\left.d_{6}\right)$ 

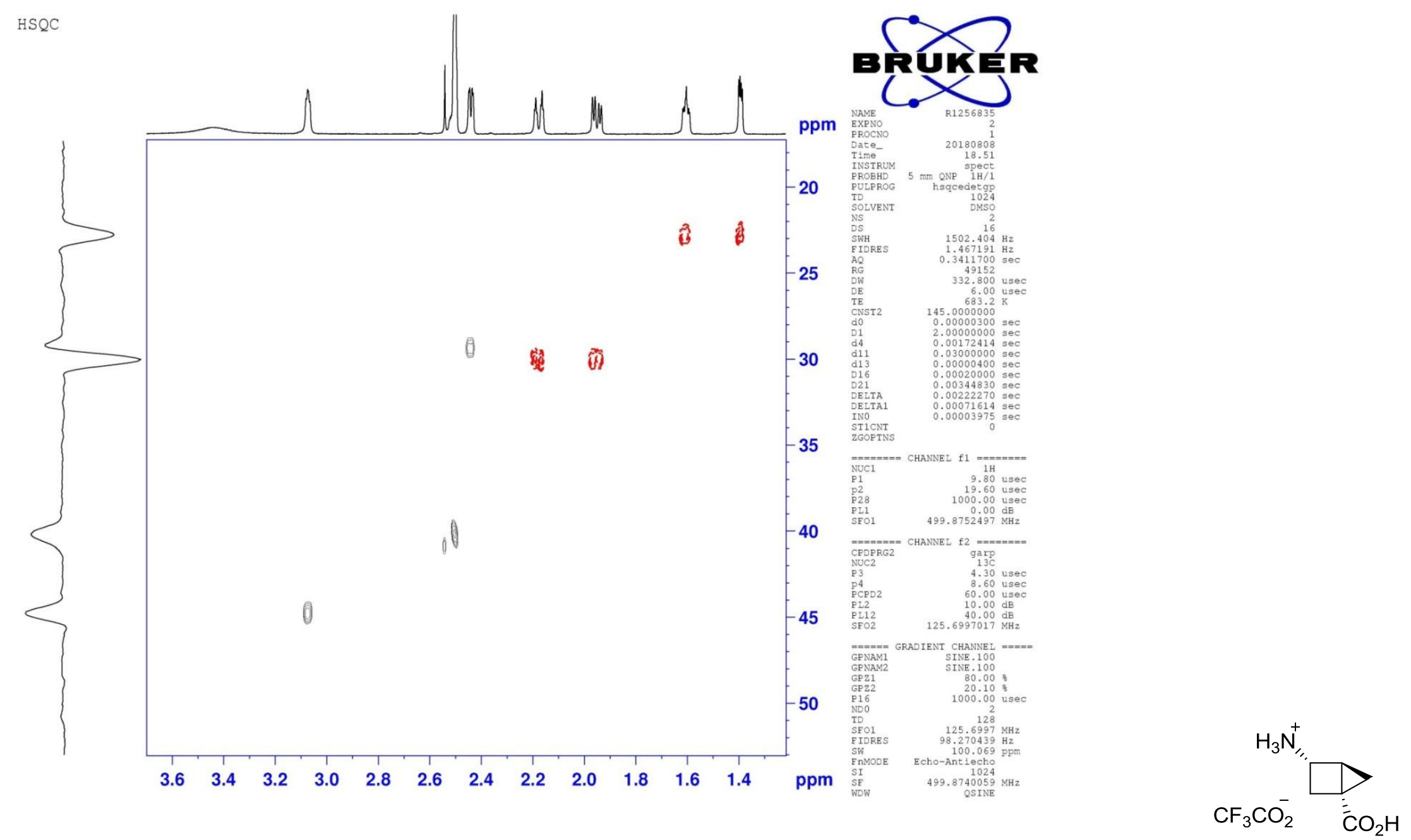

$\left(1 S^{*}, 2 S^{*}, 4 R^{*}\right)$-4-Carboxybicyclo[2.1.0]pentan-2-aminium 2,2,2-trifluoroacetate (21) (HSQC) (DMSO- $\left.d_{6}\right)$ 

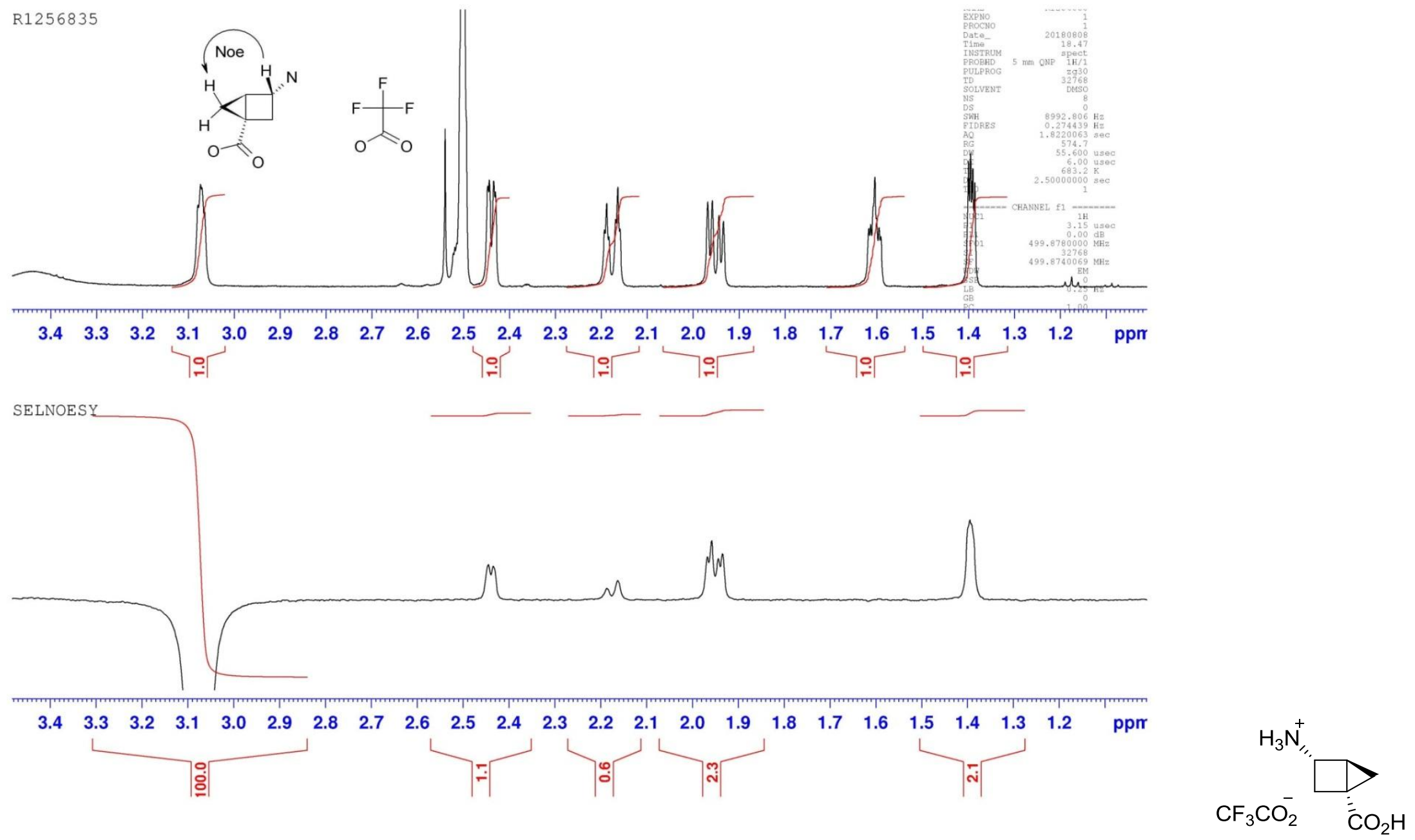

$\left(1 S^{*}, 2 S^{*}, 4 R^{*}\right)$-4-Carboxybicyclo[2.1.0]pentan-2-aminium 2,2,2-trifluoroacetate (21) (H-H NOE) (DMSO- $\left.d_{6}\right)$ 


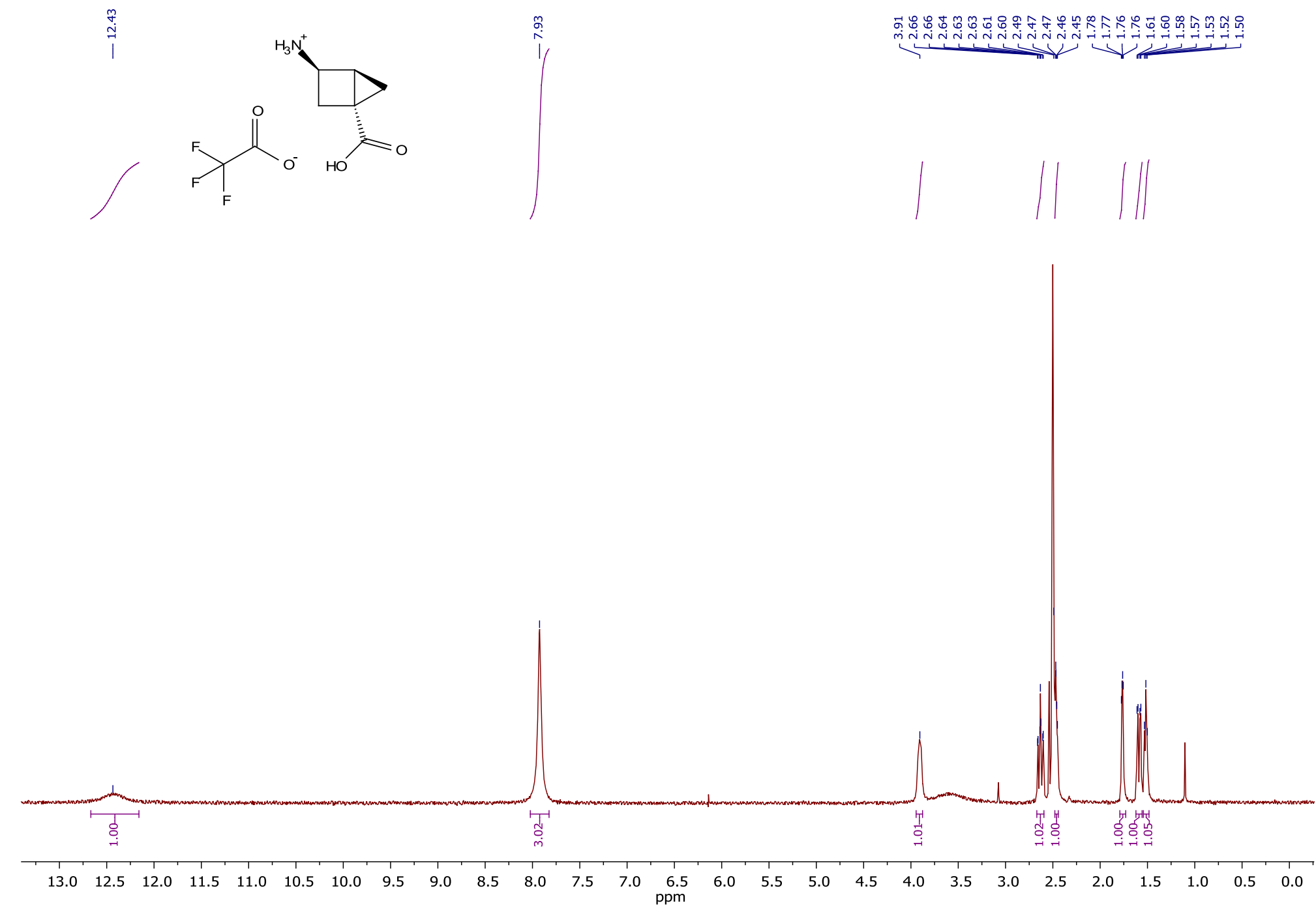

$\left(1 S^{*}, 2 R^{*}, 4 R^{*}\right)$-4-Carboxybicyclo[2.1.0]pentan-2-aminium 2,2,2-trifluoroacetate (22) ${ }^{1} \mathrm{H}$ NMR (DMSO- $\left.d_{6}\right)$ 


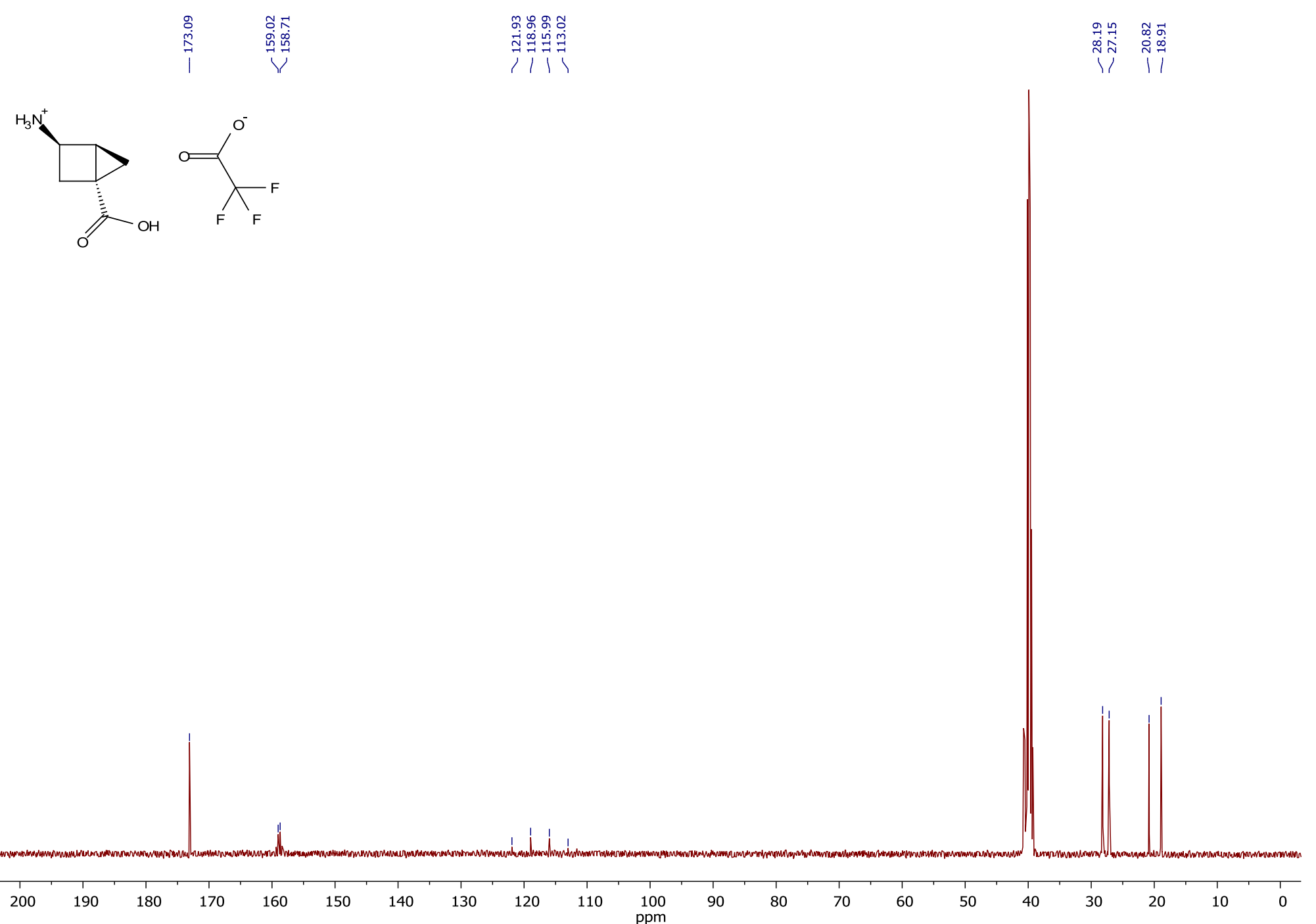

$\left(1 S^{*}, 2 R^{*}, 4 R^{*}\right)-4-C a r b o x y b i c y c l o[2.1 .0]$ pentan-2-aminium 2,2,2-trifluoroacetate (22) ${ }^{13} \mathrm{C}$ NMR (DMSO-d $)$ 


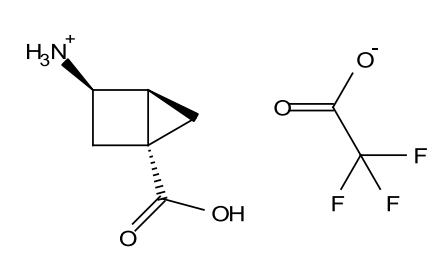

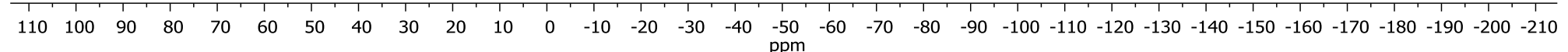
$\left(1 S^{*}, 2 R^{*}, 4 R^{*}\right)$-4-Carboxybicyclo[2.1.0]pentan-2-aminium 2,2,2-trifluoroacetate (22) ${ }^{19} \mathrm{~F}$ NMR (DMSO- $d_{6}$ ) 


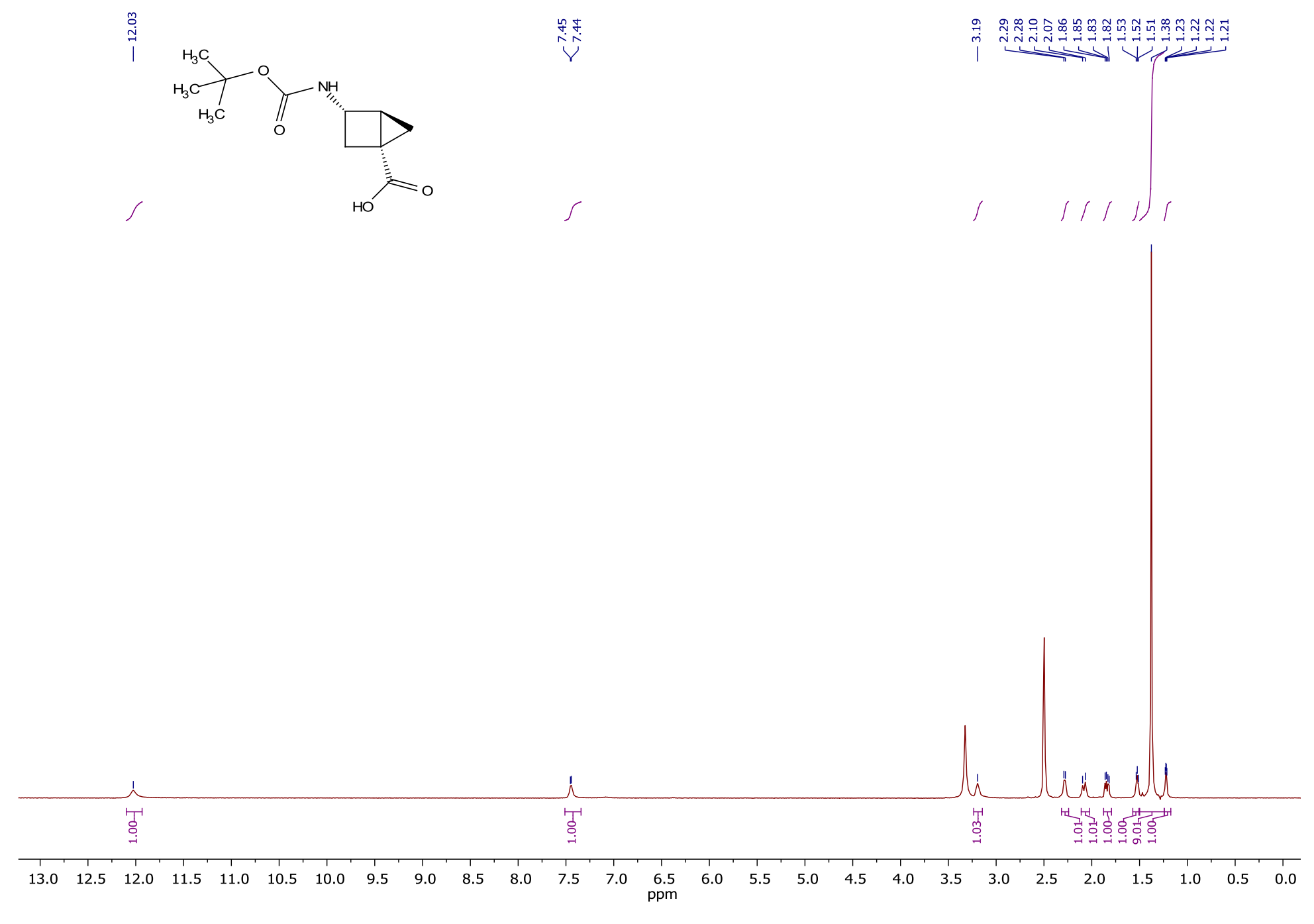

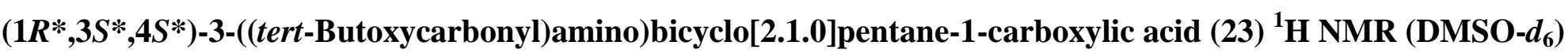




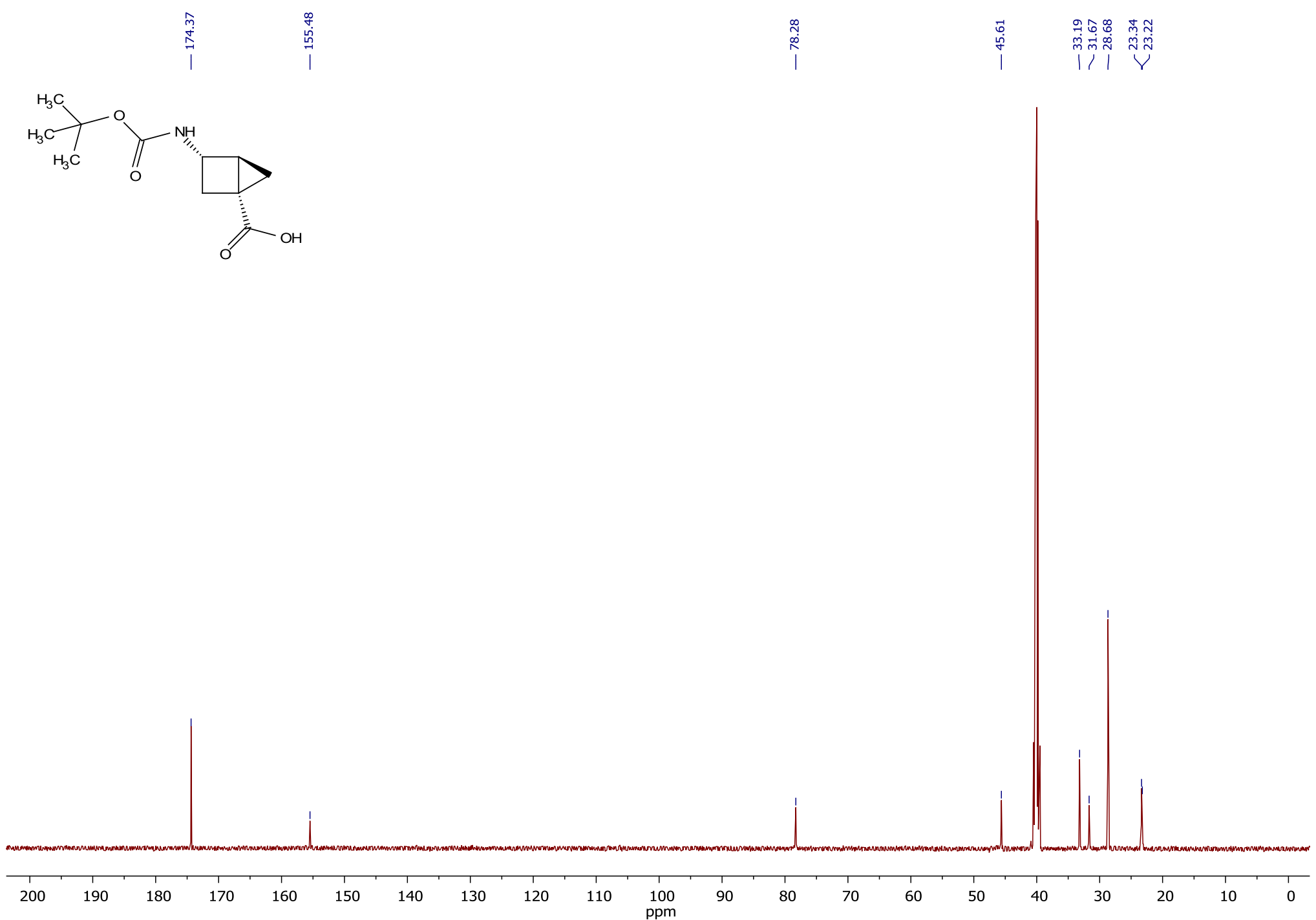

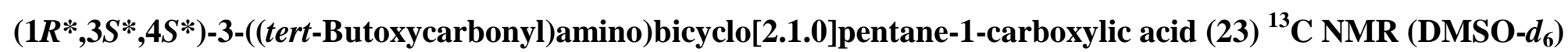




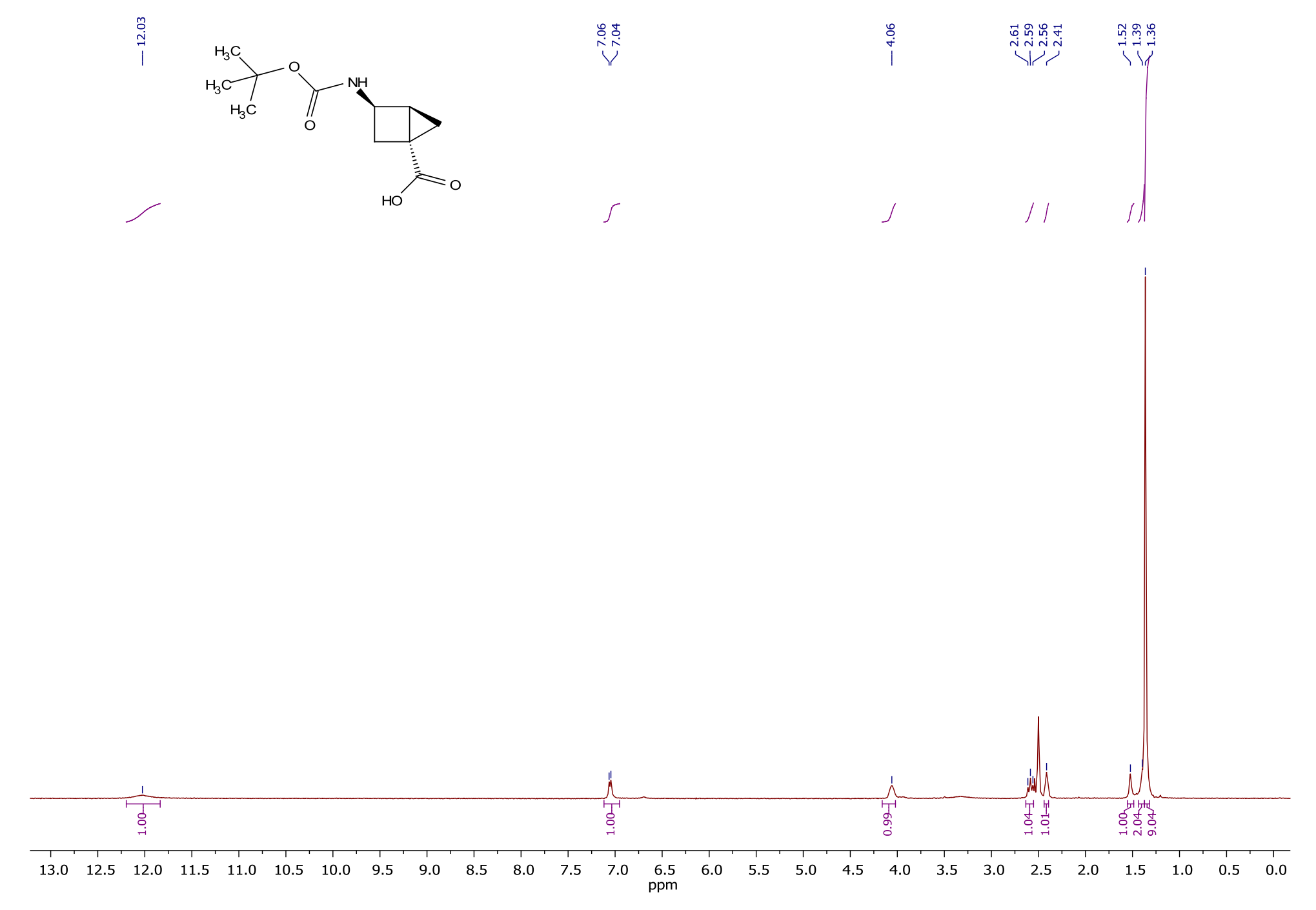

$\left(1 R^{*}, 3 R^{*}, 4 S^{*}\right)-3-\left(\left(\right.\right.$ tert-Butoxycarbonyl)amino)bicyclo[2.1.0]pentane-1-carboxylic acid (24) ${ }^{1}$ H NMR (DMSO-d $\left.d_{6}\right)$ 


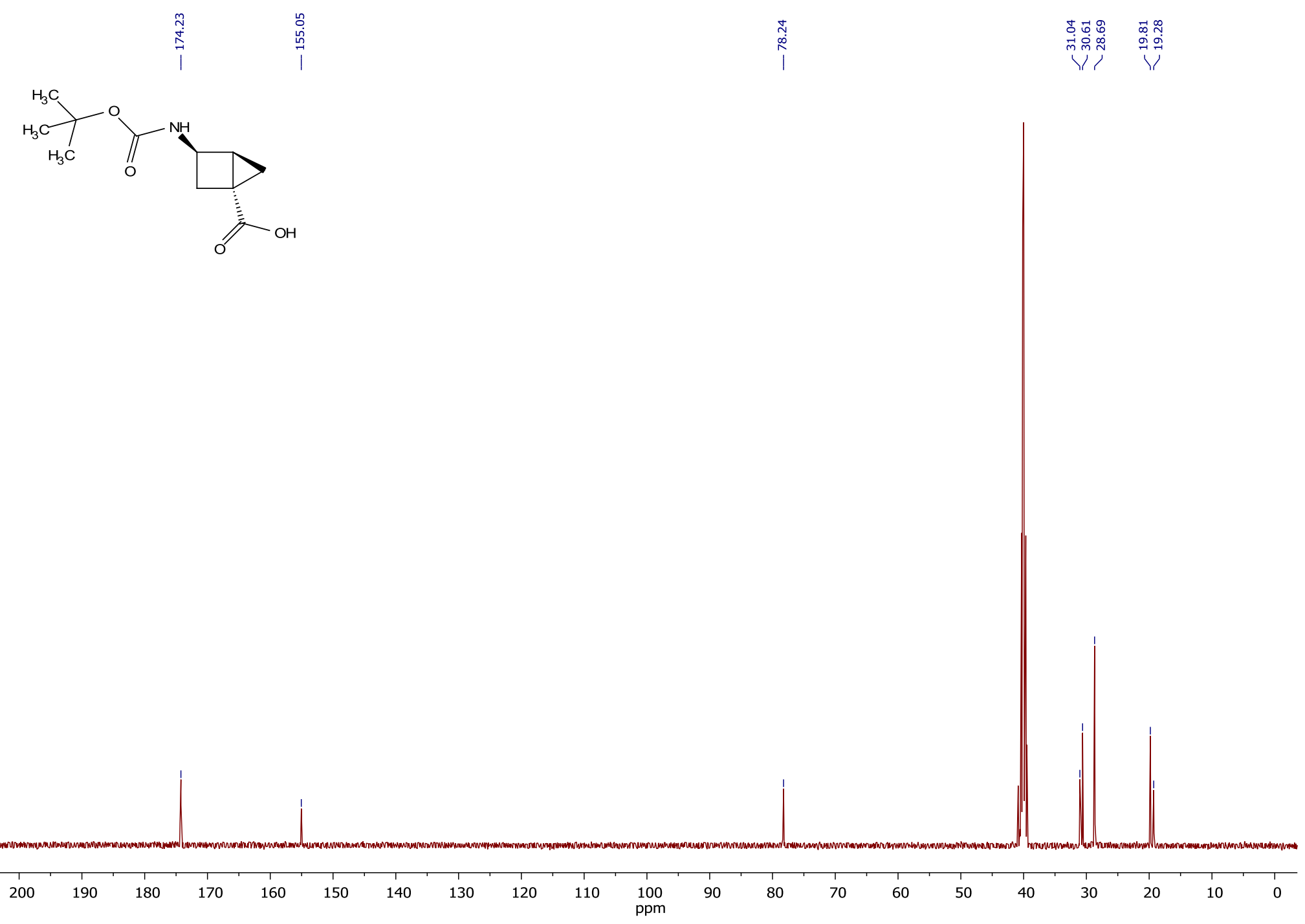

$\left(1 R^{*}, 3 R^{*}, 4 S^{*}\right)-3-((t e r t-B u t o x y c a r b o n y l) a m i n o) b i c y c l o[2.1 .0]$ pentane-1-carboxylic acid $(24){ }^{13} \mathrm{C}$ NMR (DMSO-d $)$ 

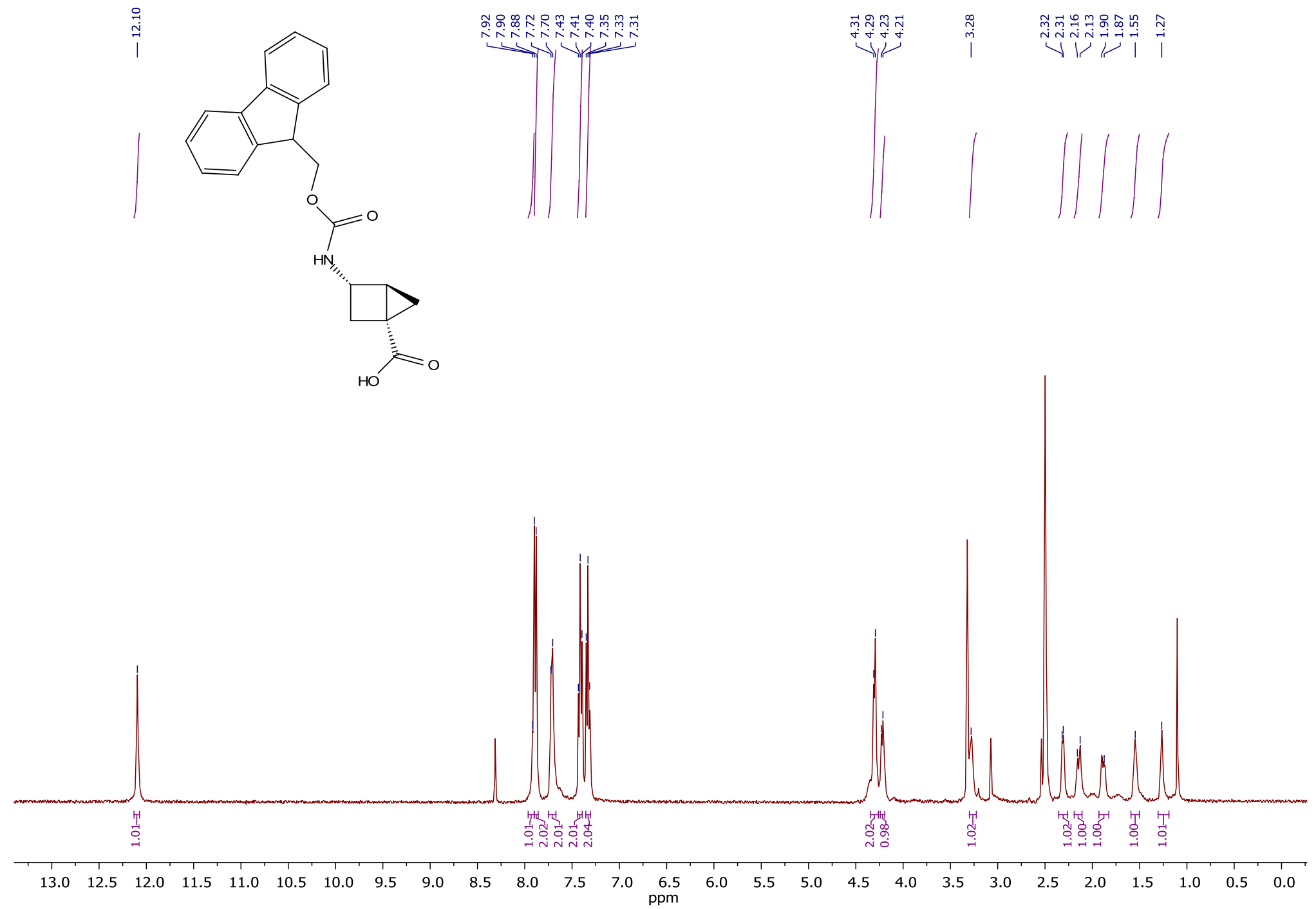

$\left(1 R^{*}, 3 S^{*}, 4 S^{*}\right)-3-\left(\left(\left(\left(9 H\right.\right.\right.\right.$-Fluoren-9-yl)methoxy)carbonyl)amino)bicyclo[2.1.0]pentane-1-carboxylic acid (25) ${ }^{1}$ H NMR (DMSO-d $)$ 


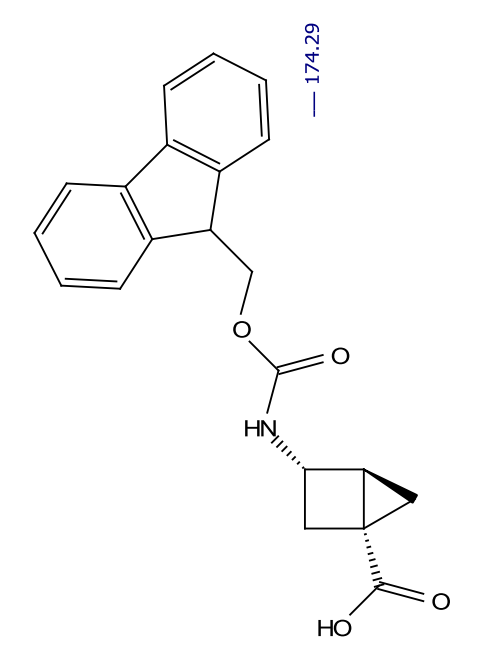

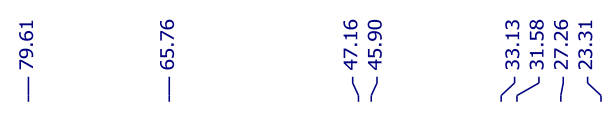

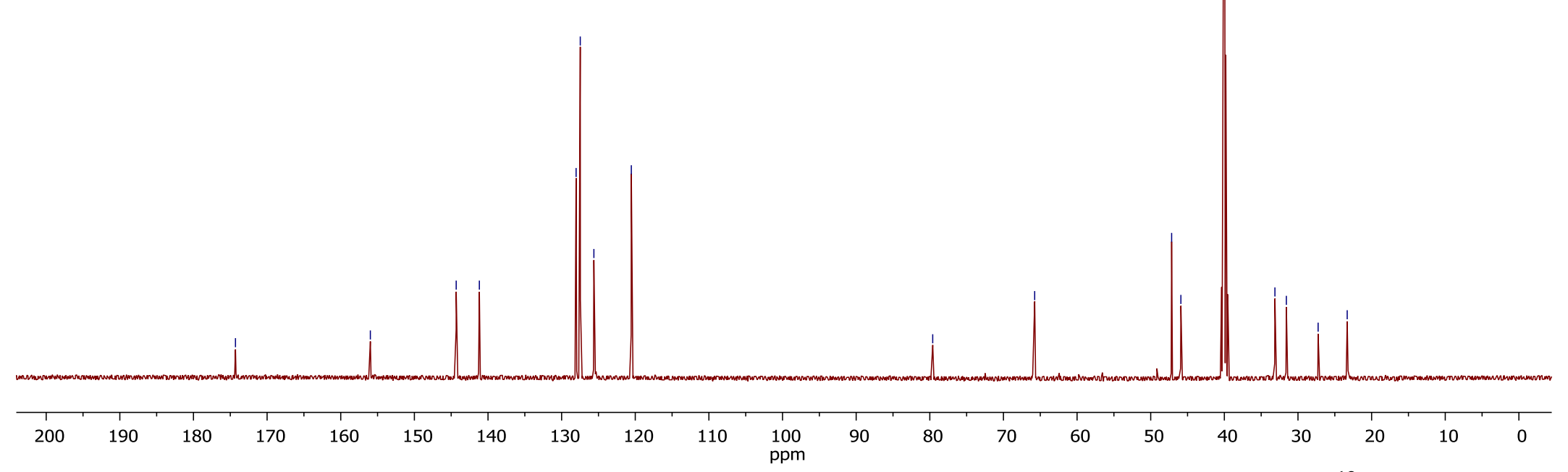

$\left(1 R^{*}, 3 S^{*}, 4 S^{*}\right)-3-\left(\left(((9 H-F l u o r e n-9-y l) m e t h o x y)\right.\right.$ carbonyl)amino)bicyclo[2.1.0]pentane-1-carboxylic acid $(25){ }^{13} \mathrm{C}$ NMR $\left(\mathrm{DMSO}-d_{6}\right)$ 

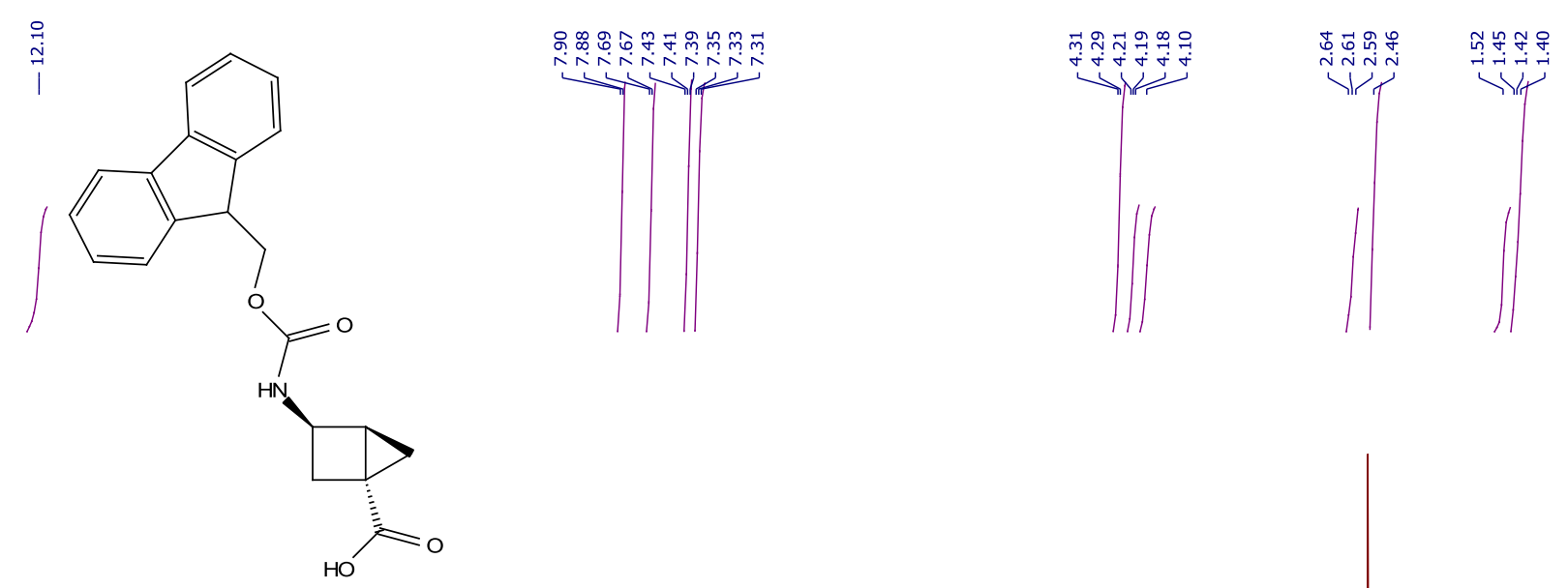

HO

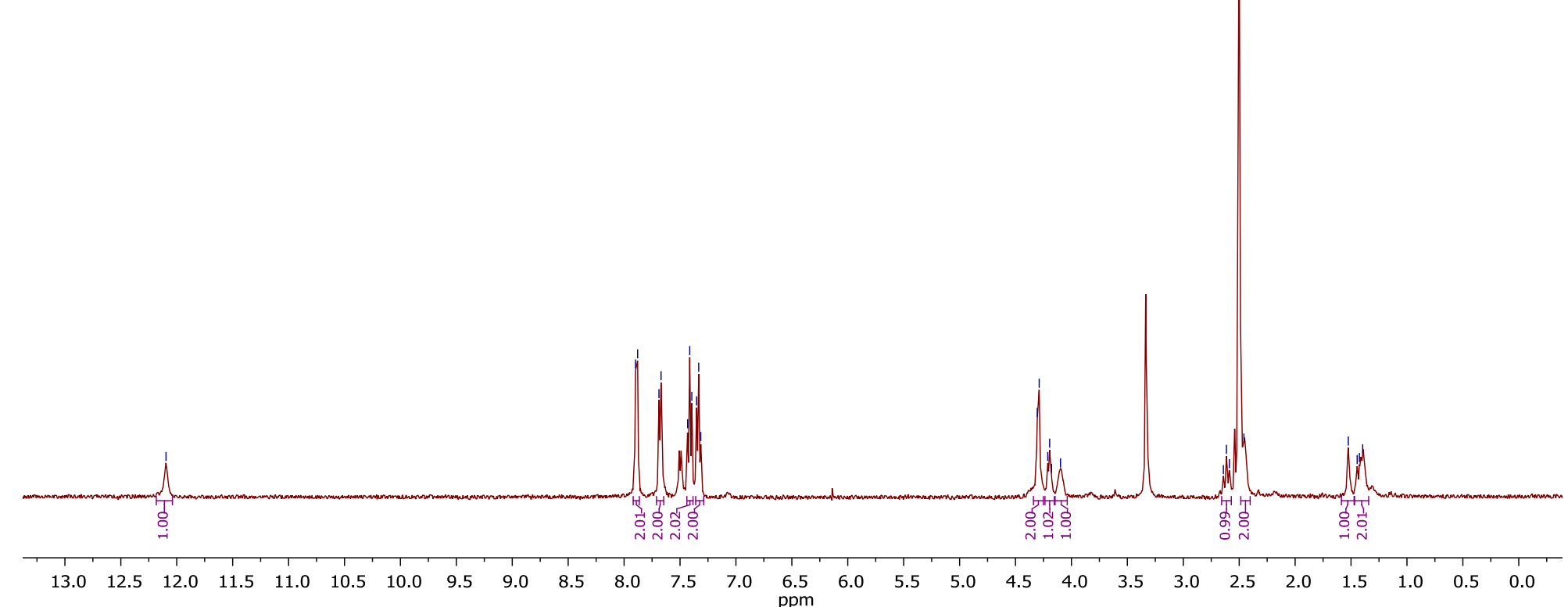

$\left(1 R^{*}, 3 R^{*}, 4 S^{*}\right)-3-\left(\left(\left(\left(9 H\right.\right.\right.\right.$-fluoren-9-yl)methoxy)carbonyl)amino)bicyclo[2.1.0]pentane-1-carboxylic acid (26) ${ }^{1}$ H NMR (DMSO- $\left.d_{6}\right)$ 

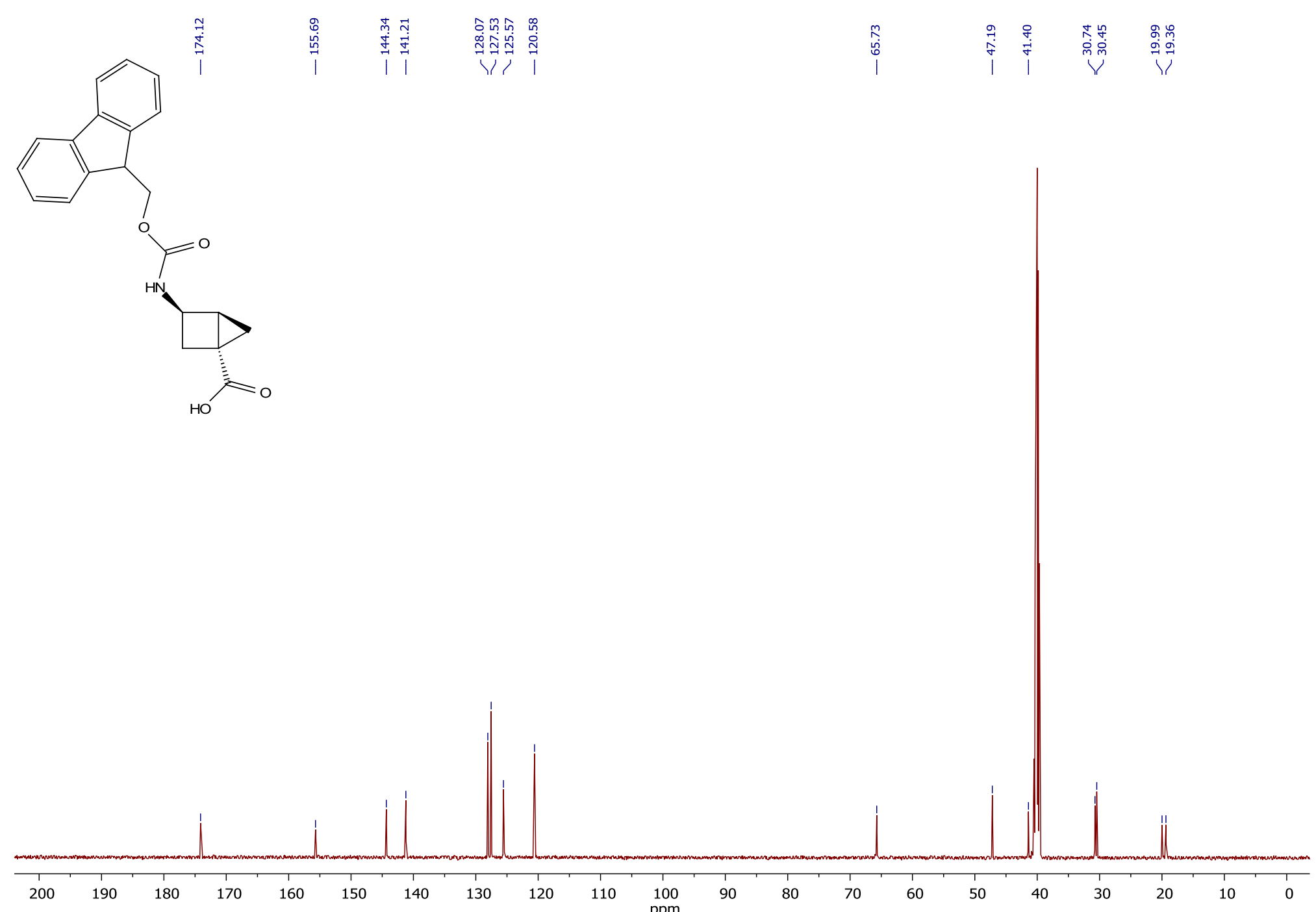

$\left(1 R^{*}, 3 R^{*}, 4 S^{*}\right)-3-\left(\left(\left(\left(9 H\right.\right.\right.\right.$-fluoren-9-yl)methoxy)carbonyl)amino)bicyclo[2.1.0]pentane-1-carboxylic acid (26) ${ }^{13} \mathrm{C}$ NMR (DMSO-d $)$ 


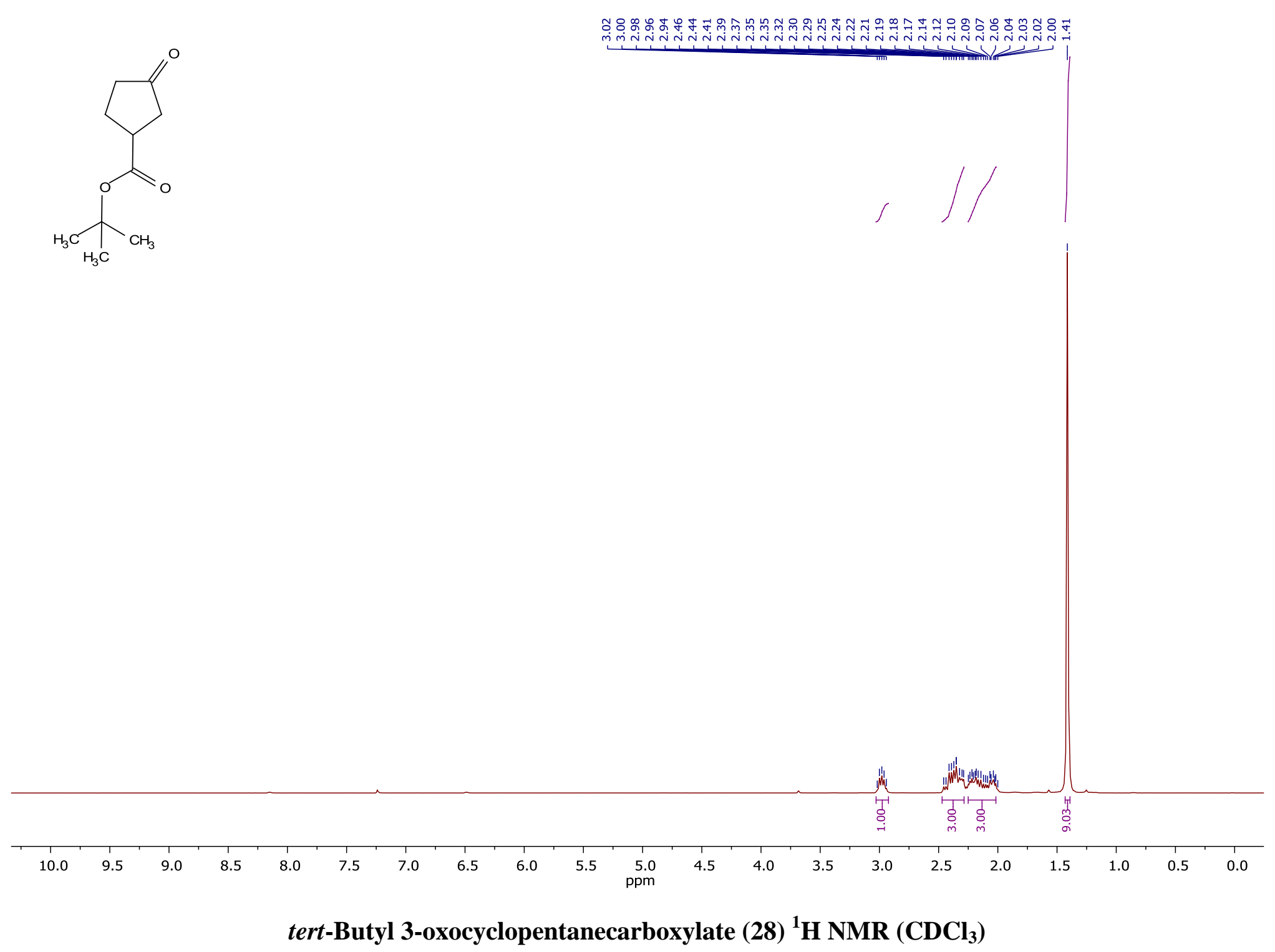



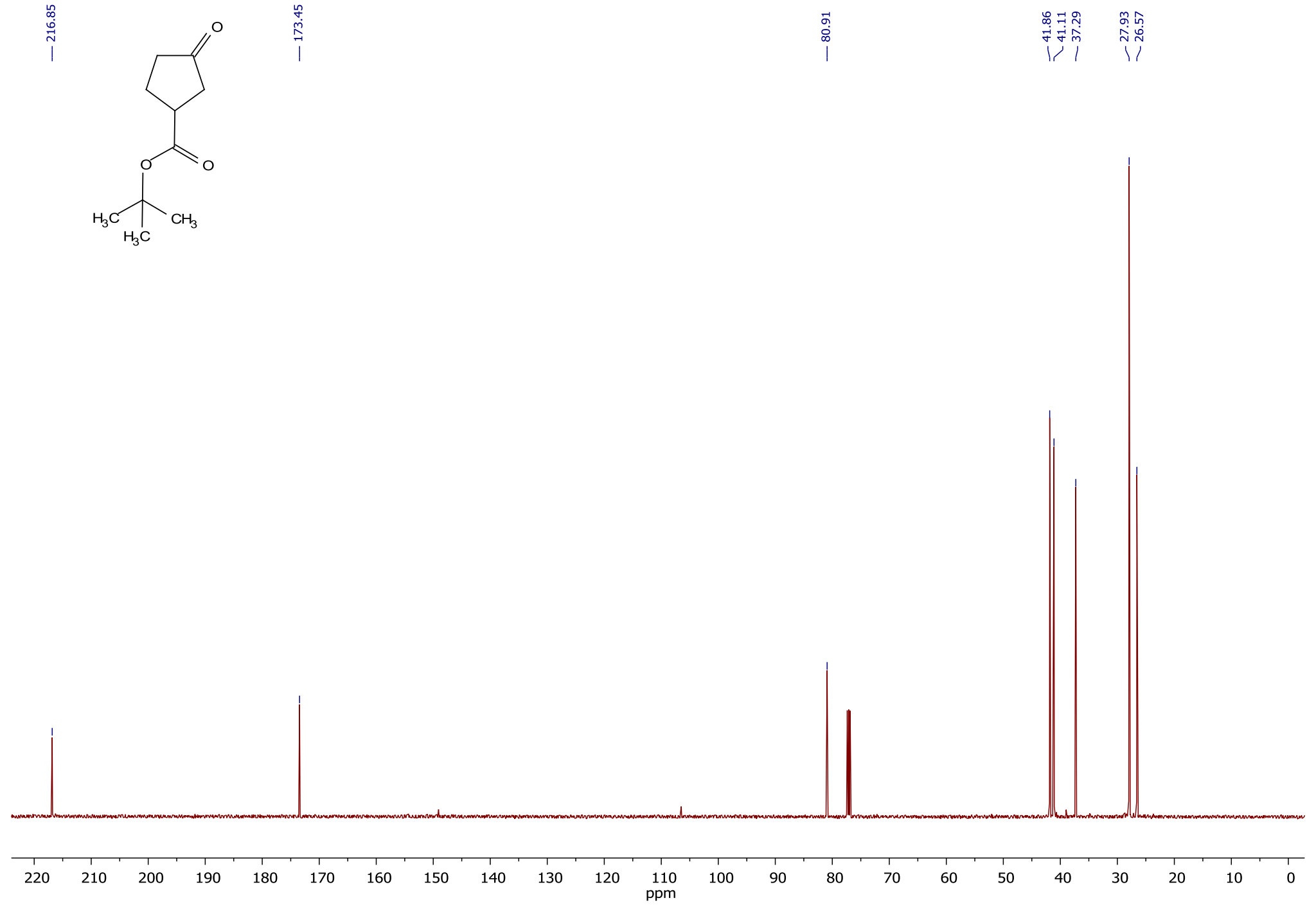

tert-Butyl 3-oxocyclopentanecarboxylate (28) ${ }^{13} \mathrm{C} \mathrm{NMR}\left(\mathrm{CDCl}_{3}\right)$ 

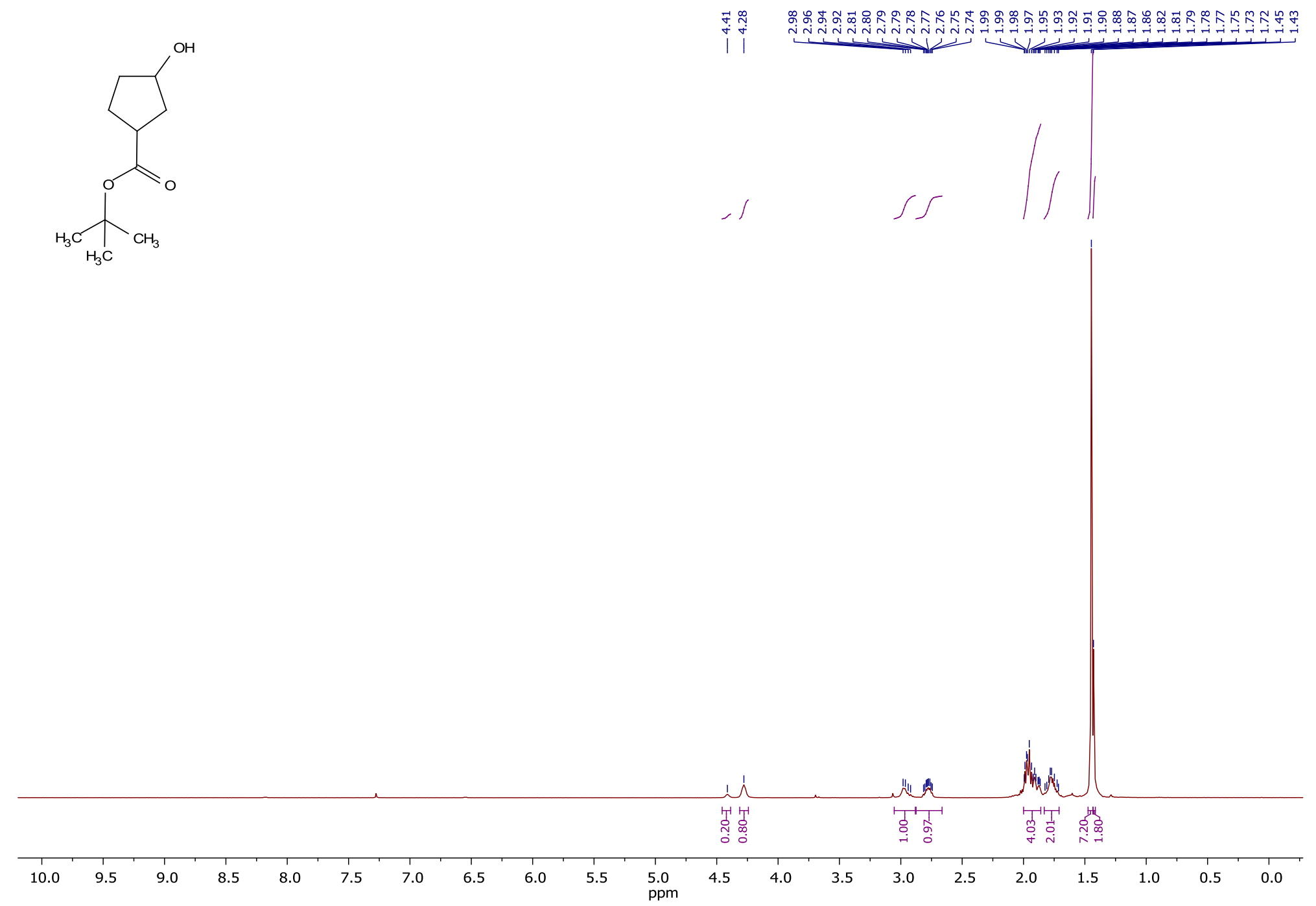

tert-Butyl 3-hydroxycyclopentanecarboxylate (29) ${ }^{1} \mathrm{H}$ NMR $\left(\mathrm{CDCl}_{3}\right)$ 


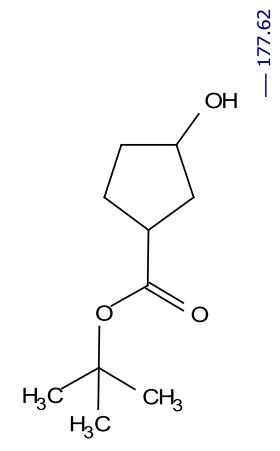

tert-Butyl 3-hydroxycyclopentanecarboxylate (29) ${ }^{13} \mathrm{C} \mathrm{NMR}\left(\mathrm{CDCl}_{3}\right)$ 


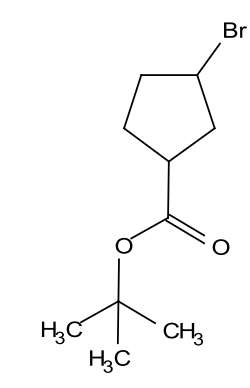

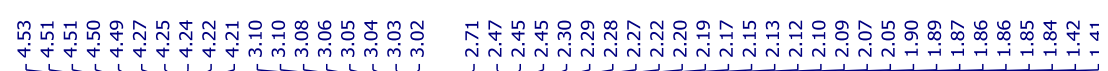
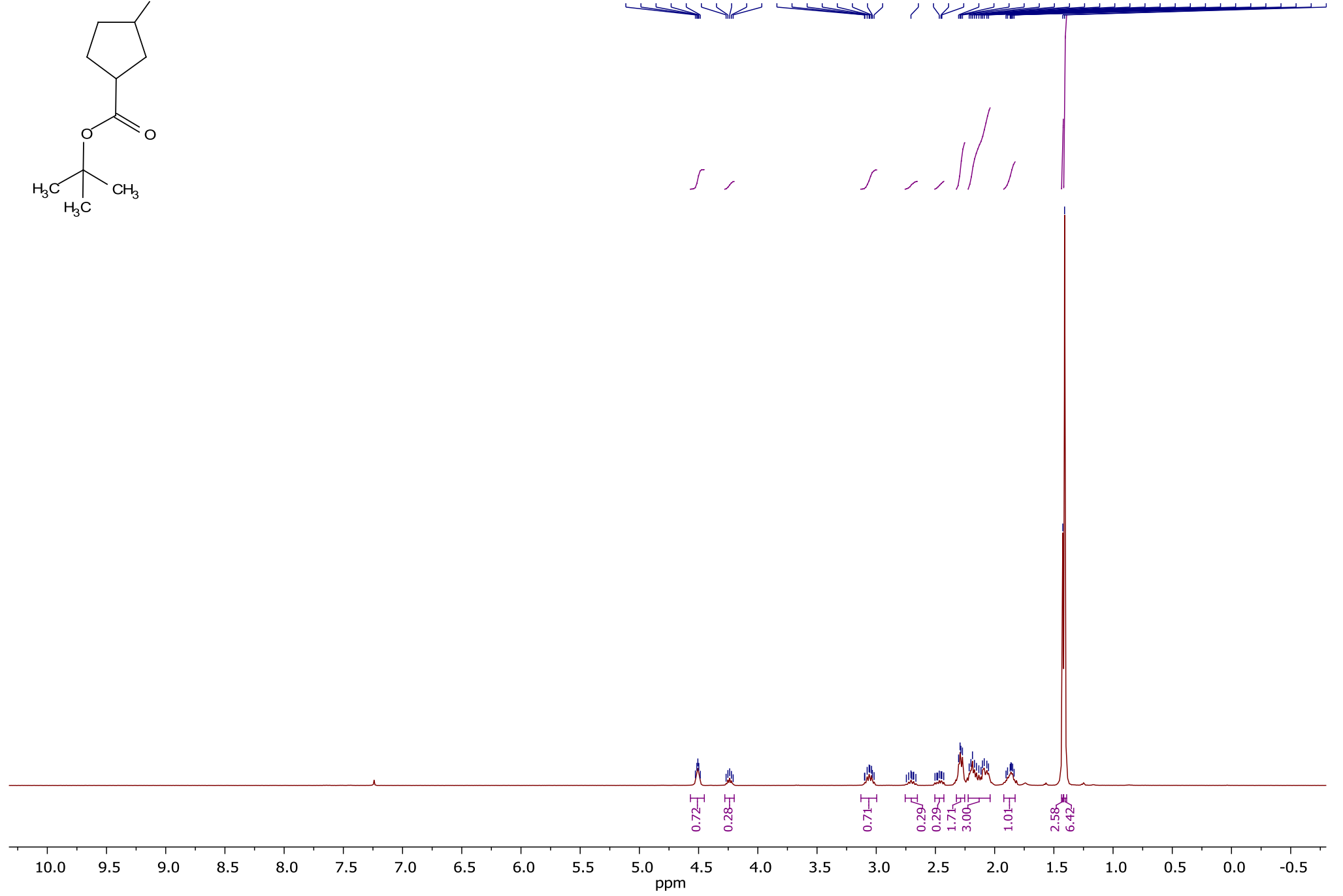

tert-Butyl 3-bromocyclopentanecarboxylate (30) ${ }^{1} \mathrm{H}$ NMR $\left(\mathrm{CDCl}_{3}\right)$ 

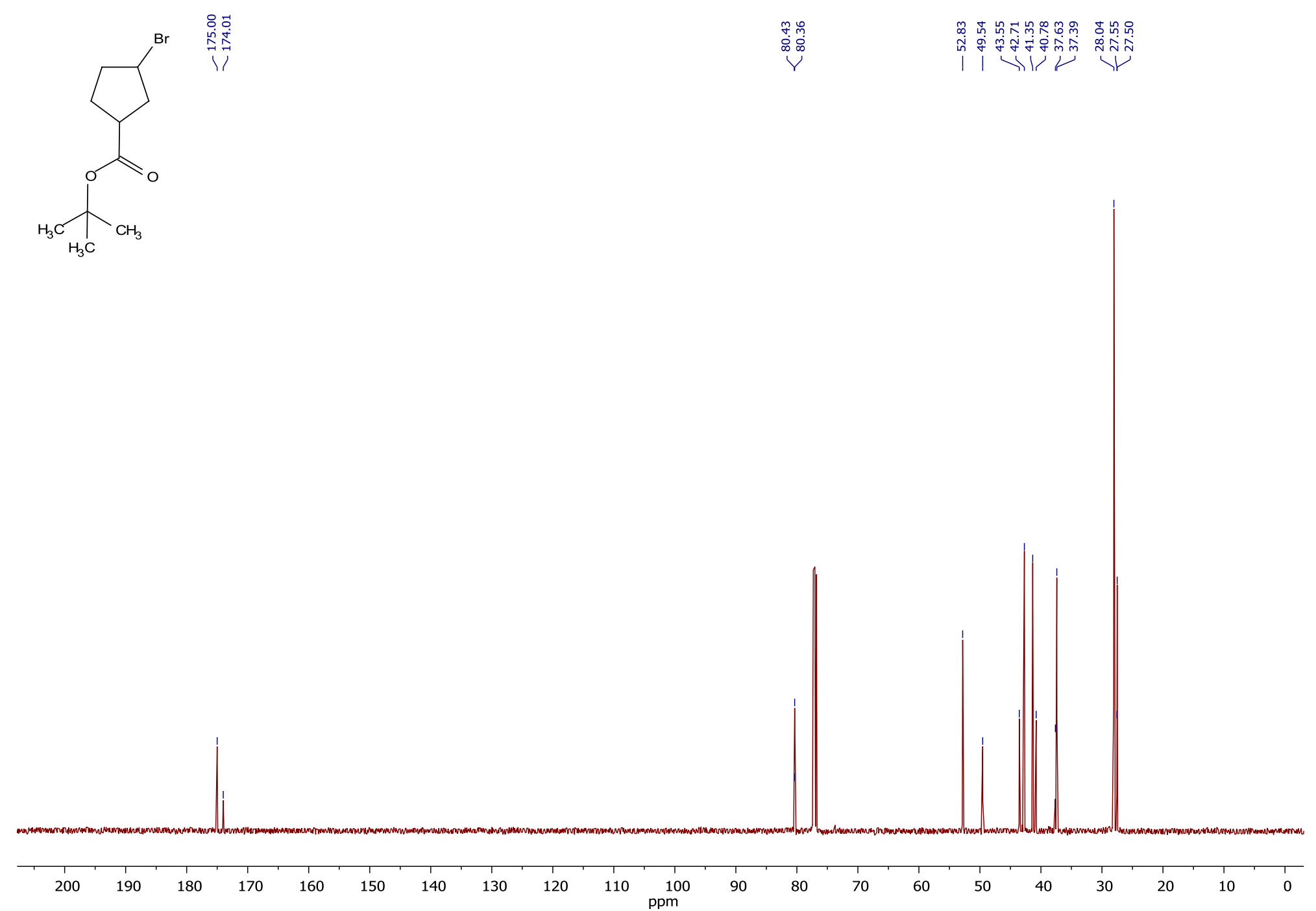

tert-Butyl 3-bromocyclopentanecarboxylate (30) ${ }^{13} \mathrm{C} \mathrm{NMR}\left(\mathrm{CDCl}_{3}\right)$ 


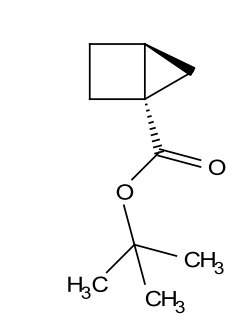

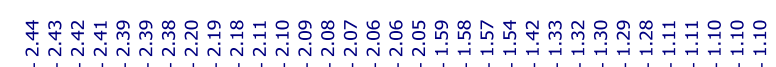

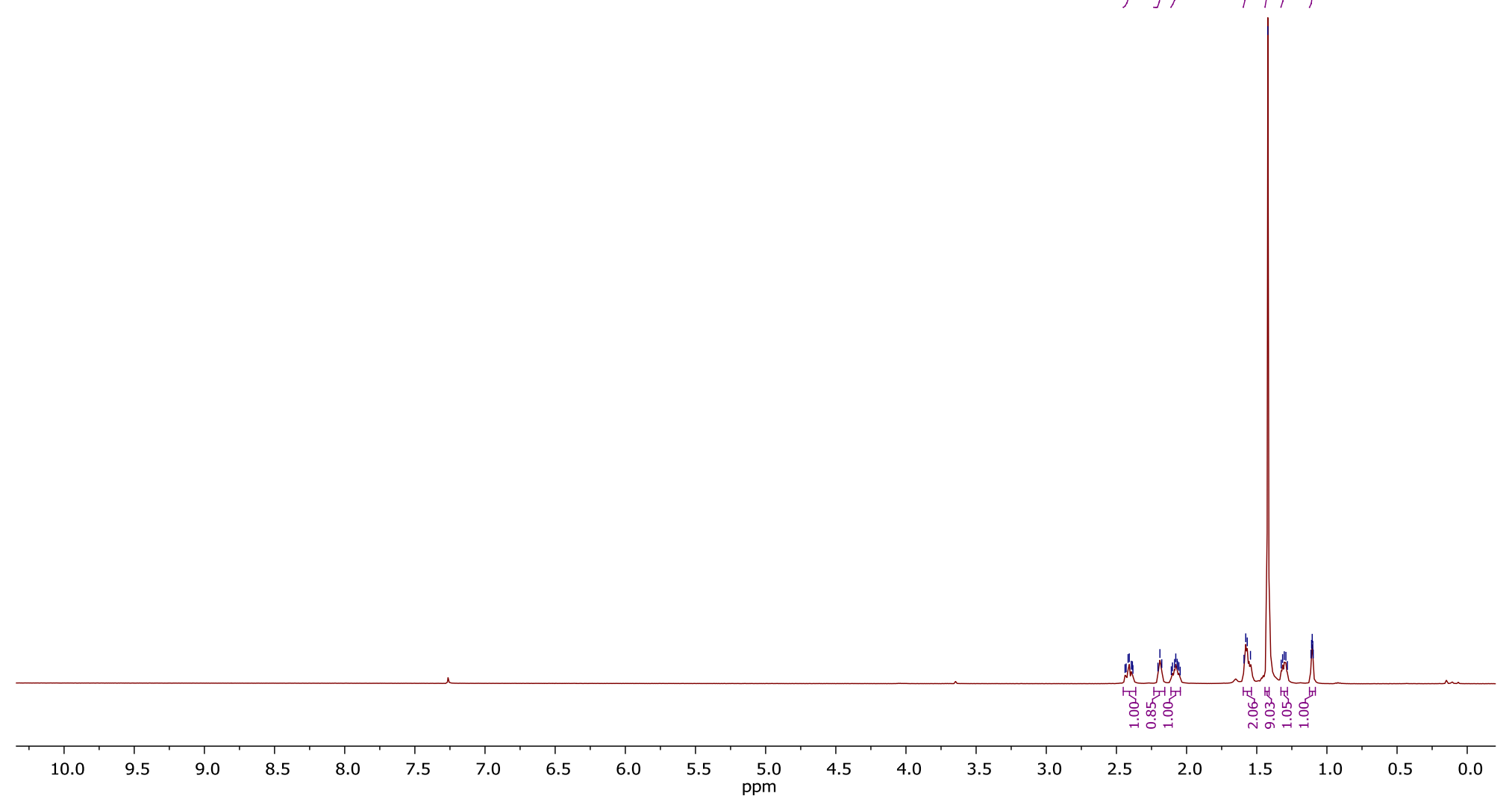

tert-Butyl bicyclo[2.1.0]pentane-1-carboxylate (31) ${ }^{1} \mathrm{H}$ NMR $\left(\mathrm{CDCl}_{3}\right)$ 


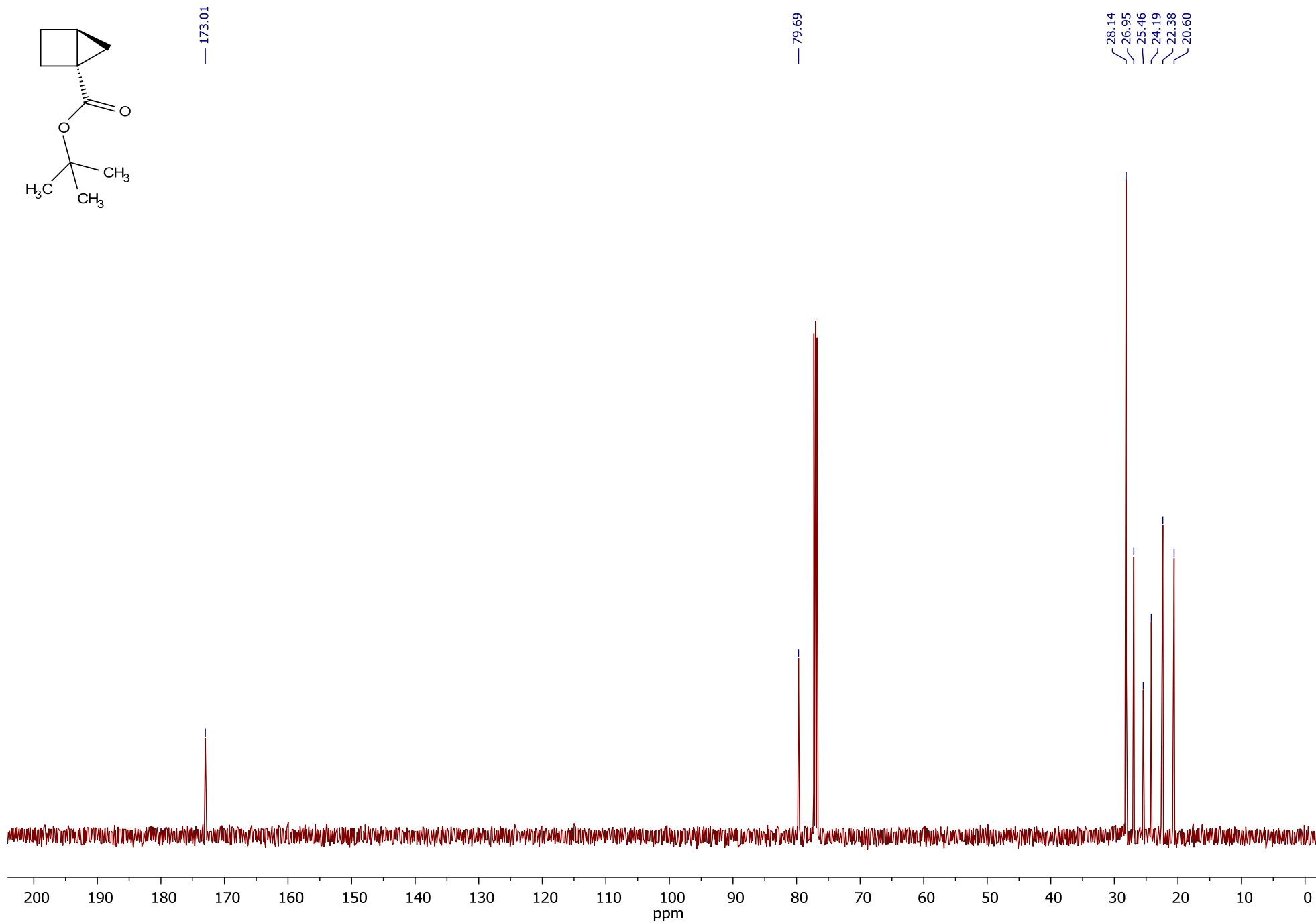

tert-Butyl bicyclo[2.1.0]pentane-1-carboxylate (31) ${ }^{1} \mathrm{H} \mathrm{NMR}\left(\mathrm{CDCl}_{3}\right)$ 


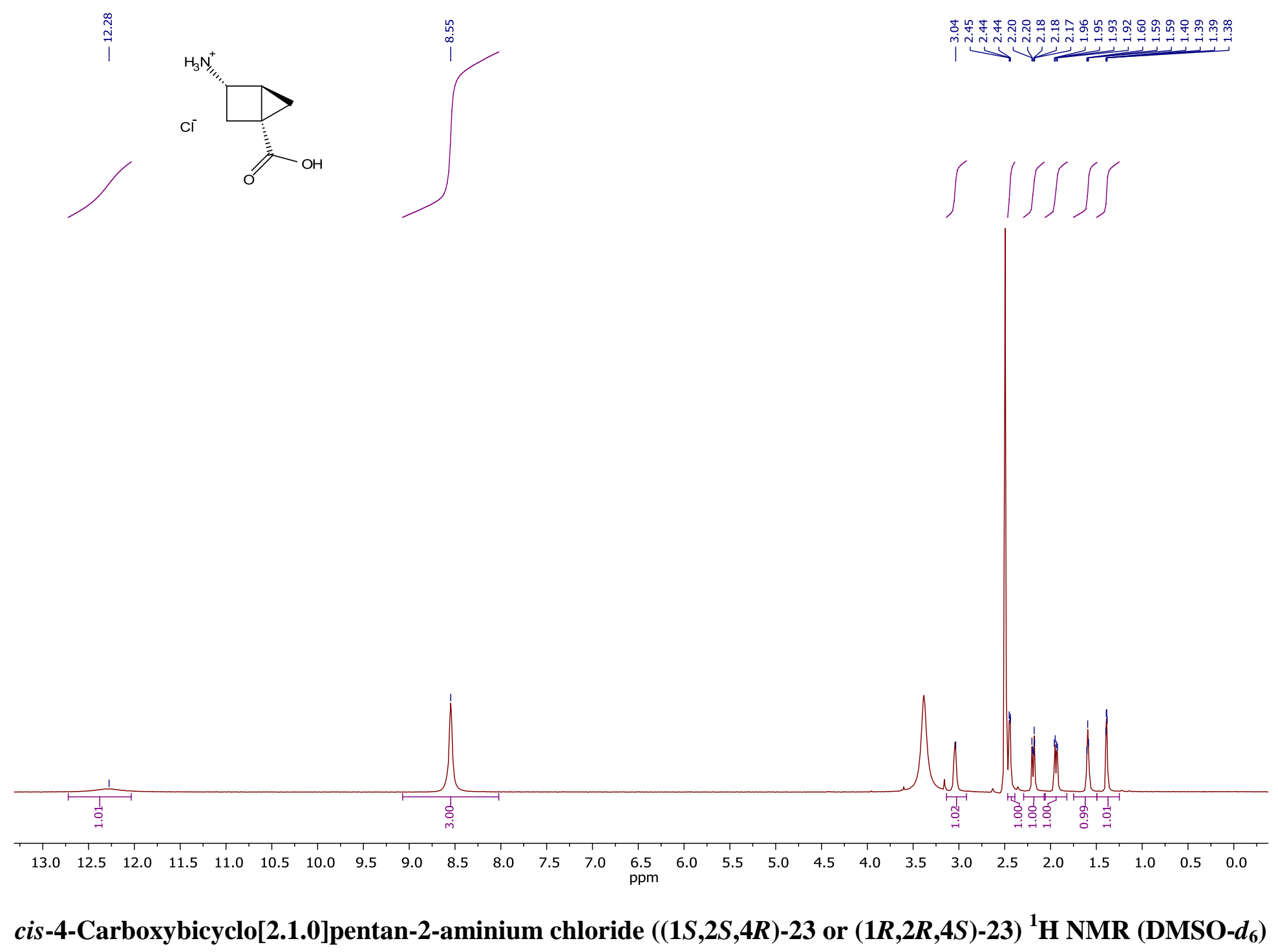



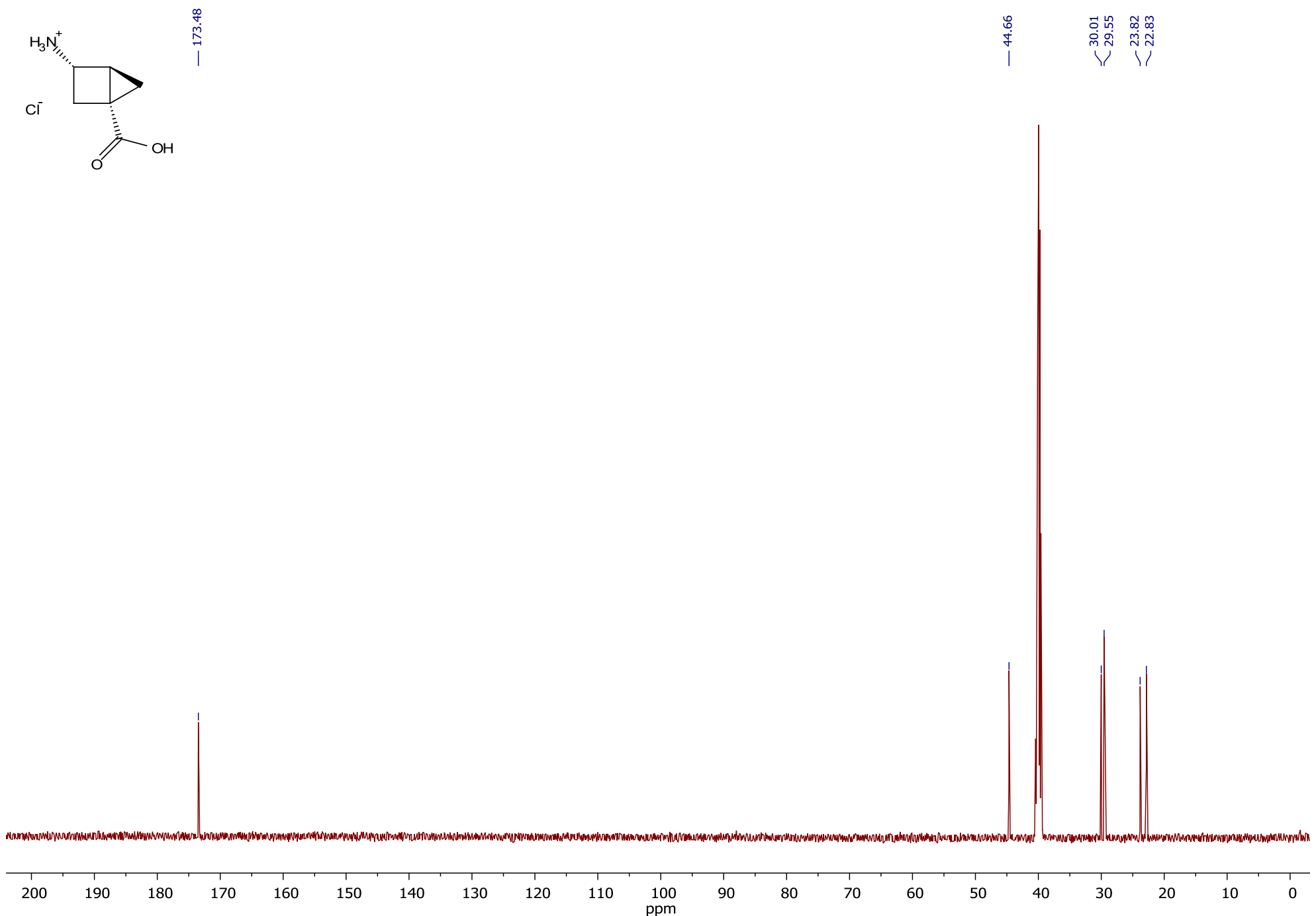

cis-4-Carboxybicyclo[2.1.0]pentan-2-aminium chloride $((1 S, 2 S, 4 R)-23$ or $(1 R, 2 R, 4 S)-23){ }^{13} \mathrm{C}$ NMR $($ DMSO-d $)$ 

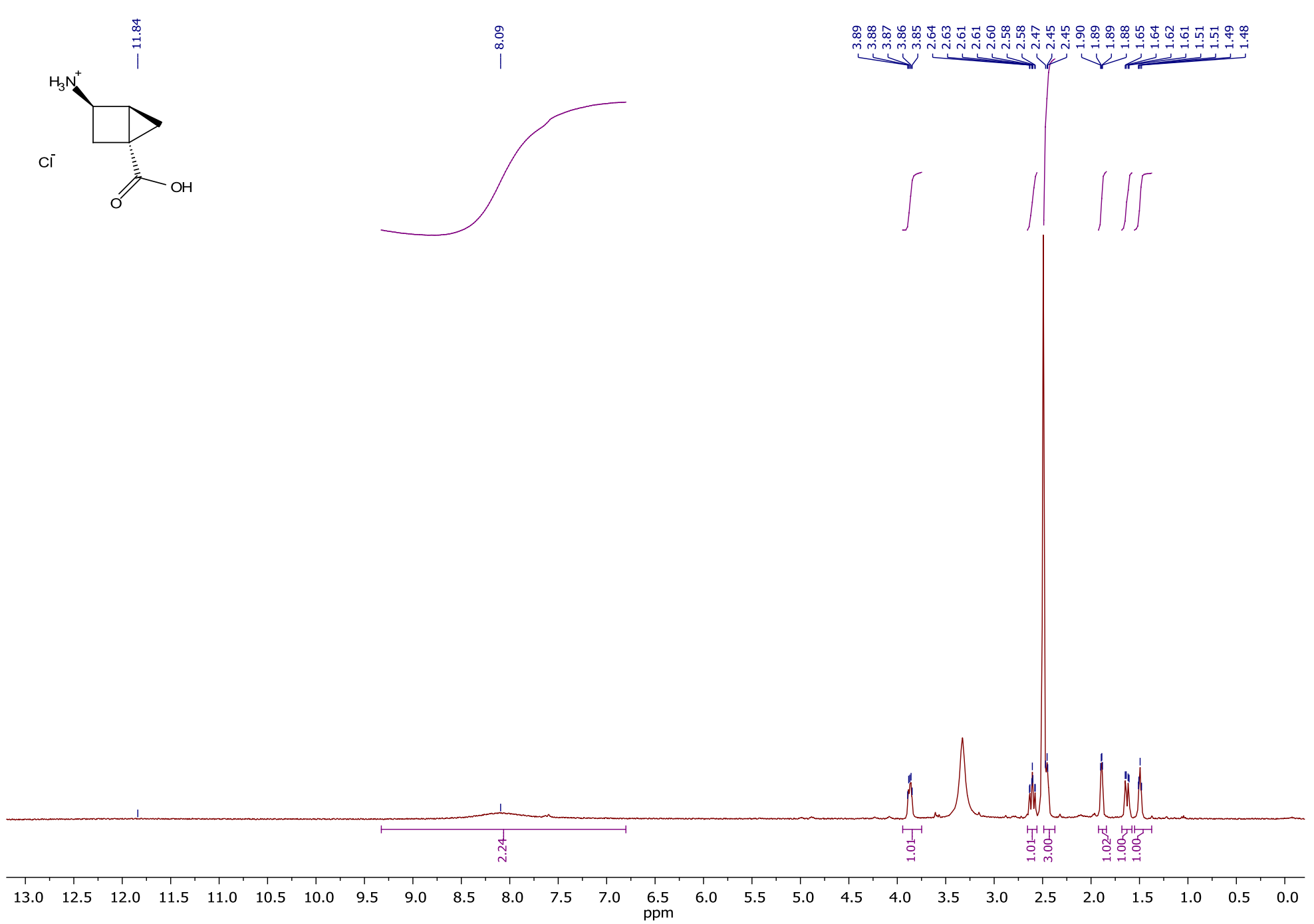

trans-4-Carboxybicyclo[2.1.0]pentan-2-aminium chloride $((1 S, 2 R, 4 R)-24$ or $(1 R, 2 S, 4 S)-24){ }^{1} \mathrm{H}$ NMR $\left(\mathrm{DMSO}-d_{6}\right)$ 

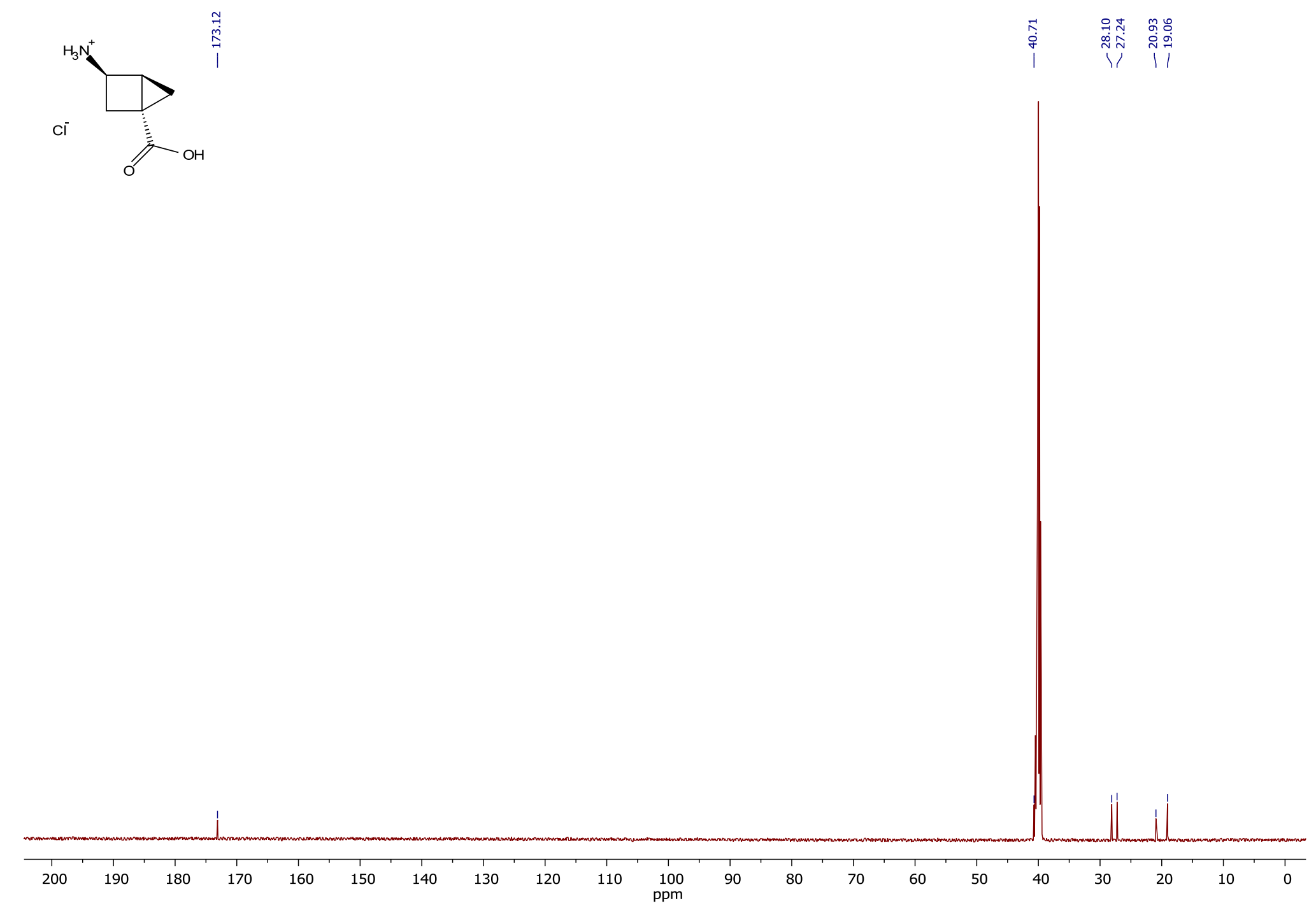

trans-4-Carboxybicyclo[2.1.0]pentan-2-aminium chloride $((1 S, 2 R, 4 R)-24$ or $(1 R, 2 S, 4 S)-24){ }^{13} \mathrm{C}$ NMR $\left(\mathrm{DMSO}-d_{6}\right)$ 


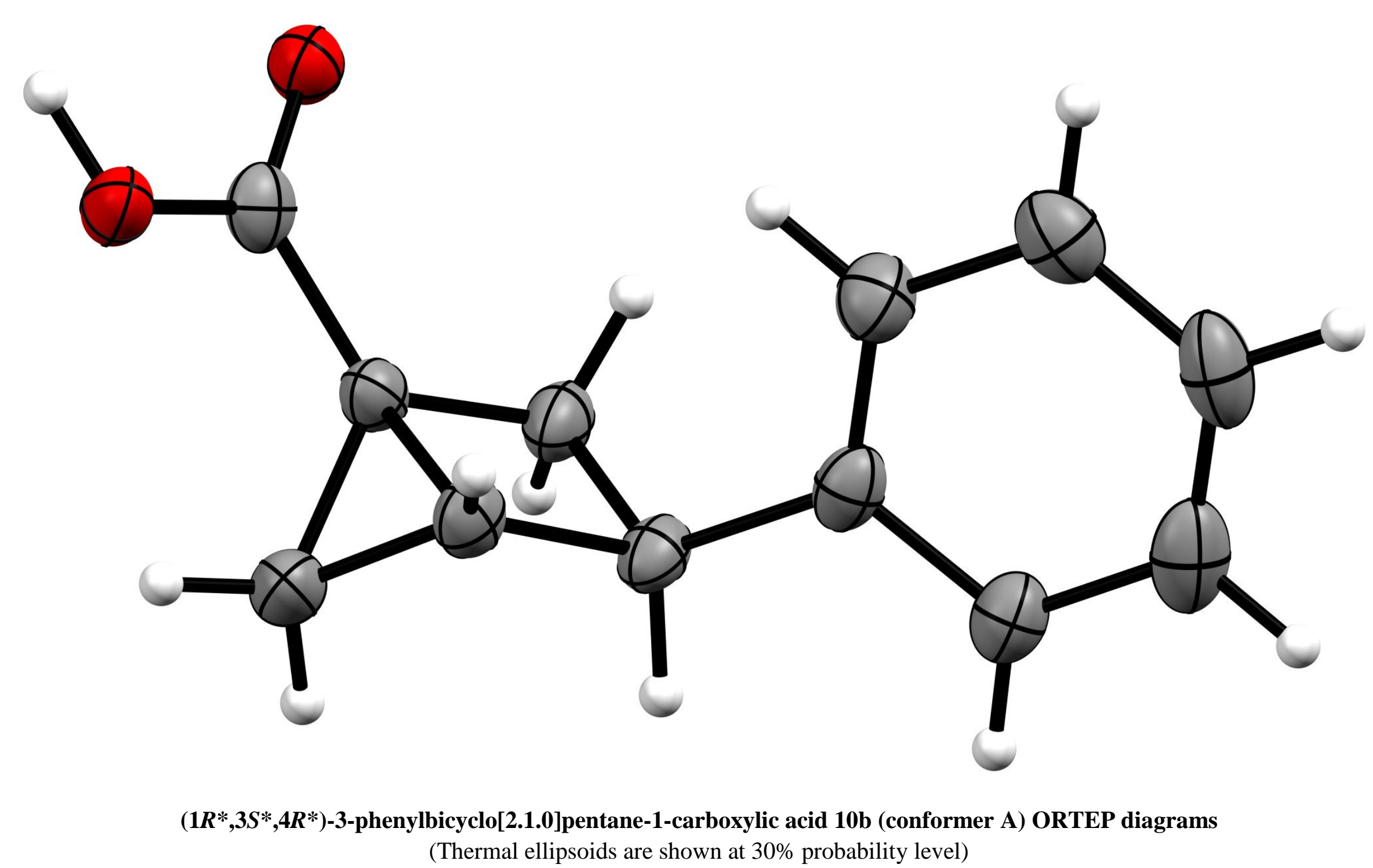




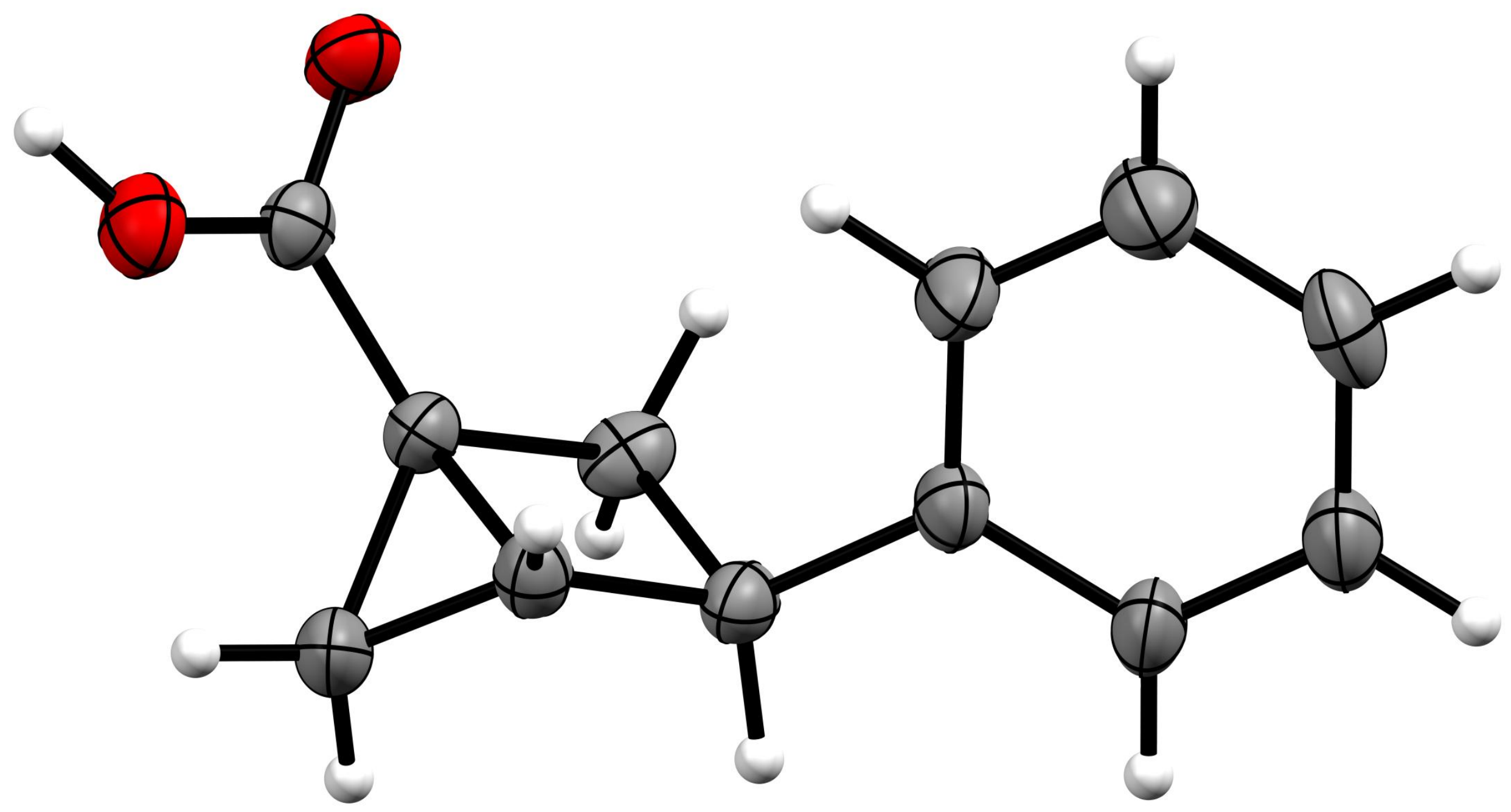

$\left(1 R^{*}, 3 S^{*}, 4 R^{*}\right)$-3-phenylbicyclo[2.1.0]pentane-1-carboxylic acid 10b (conformer B) ORTEP diagrams (Thermal ellipsoids are shown at $30 \%$ probability level) 


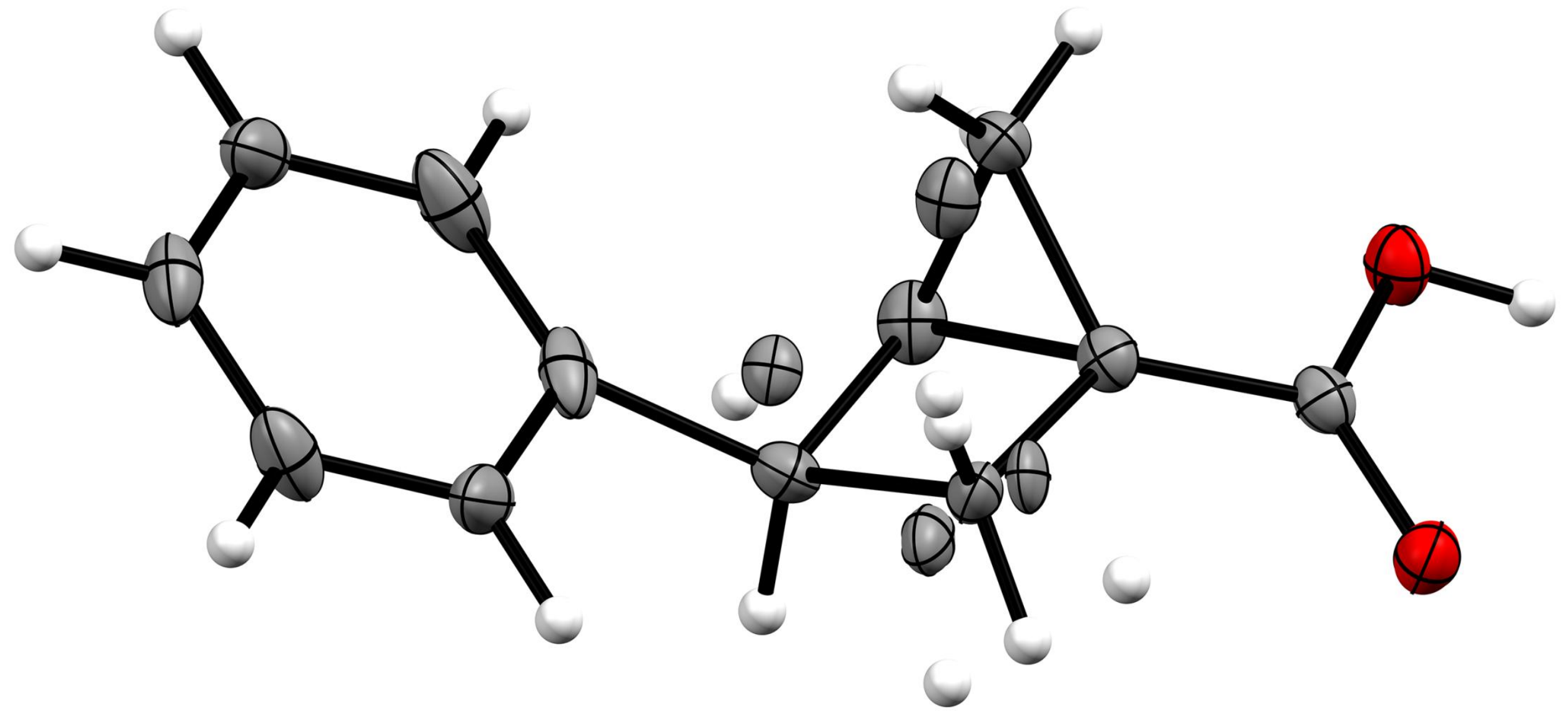

$\left(1 R^{*}, 3 R^{*}, 4 R^{*}\right)$-3-phenylbicyclo[2.1.0]pentane-1-carboxylic acid 17b ORTEP diagrams

(Thermal ellipsoids are shown at $30 \%$ probability level) 


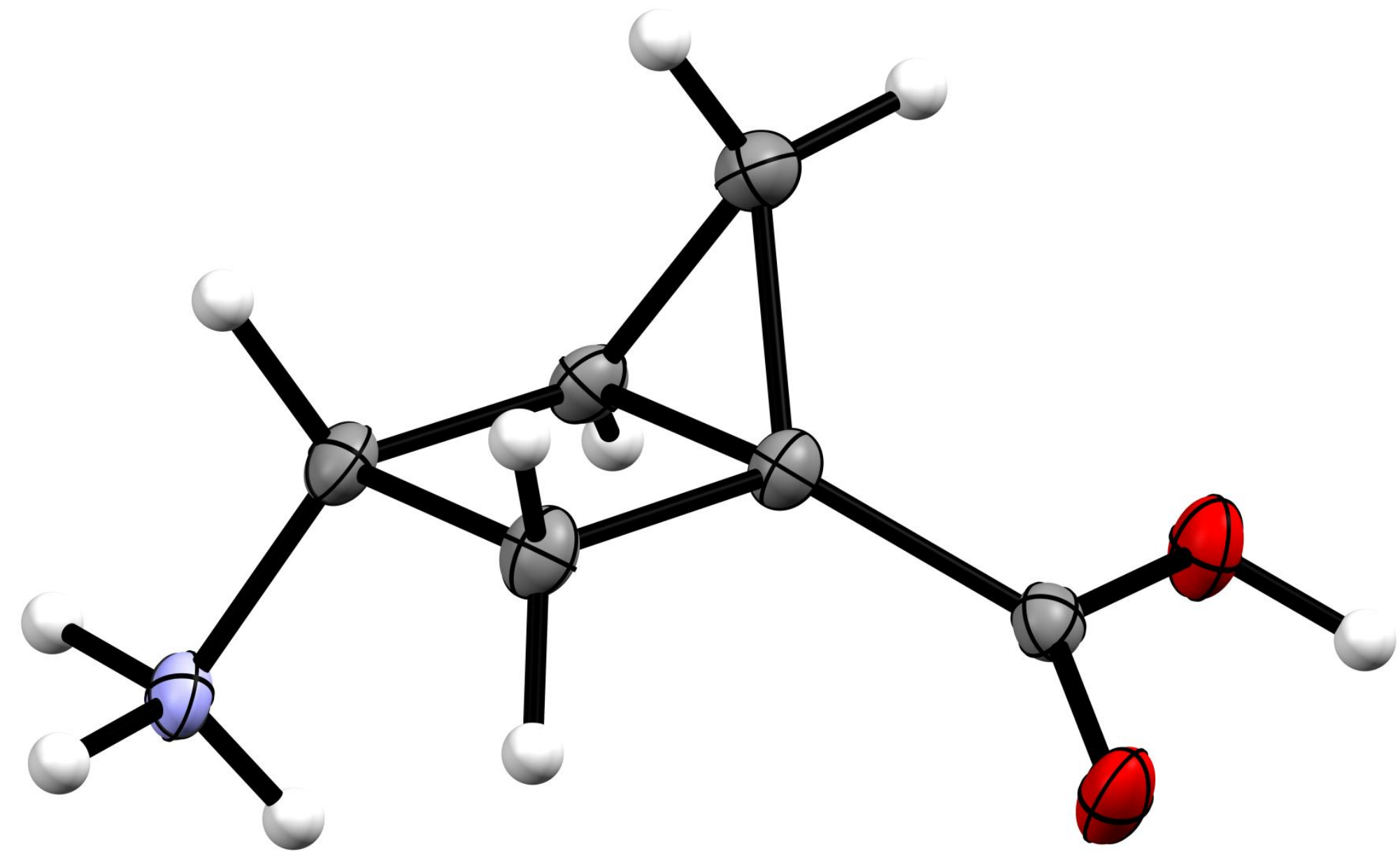

$(1 S, 2 S, 4 R)$-3-aminobicyclo[2.1.0]pentane-1-carboxylic acid hydrochloride (1S,2S,4R)-21 ORTEP diagrams (Thermal ellipsoids are shown at 30\% probability level) 


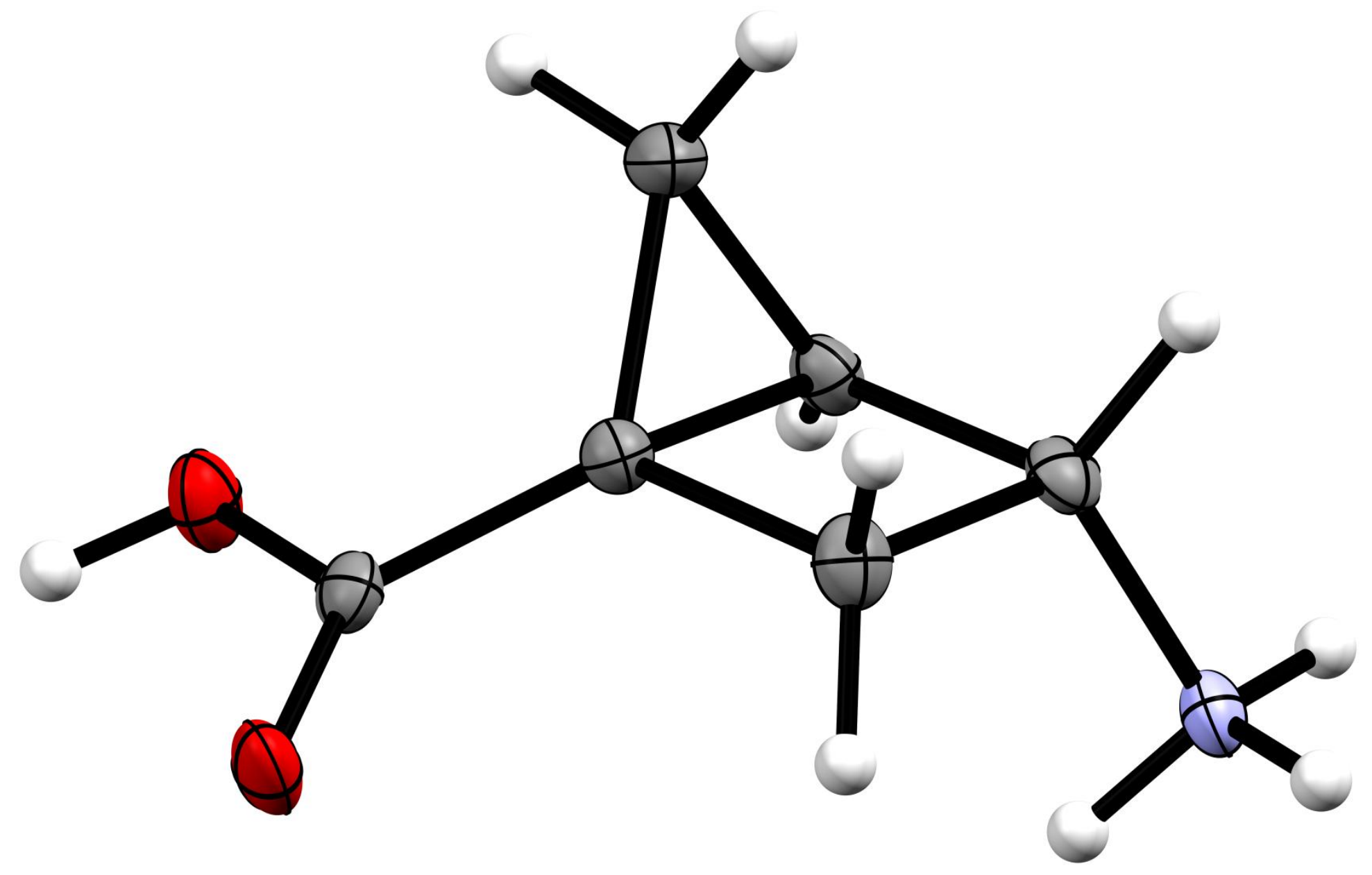

$(1 R, 2 R, 4 S)$-3-aminobicyclo[2.1.0]pentane-1-carboxylic acid hydrochloride $(1 R, 2 R, 4 S)$-21 ORTEP diagrams

(Thermal ellipsoids are shown at $30 \%$ probability level) 


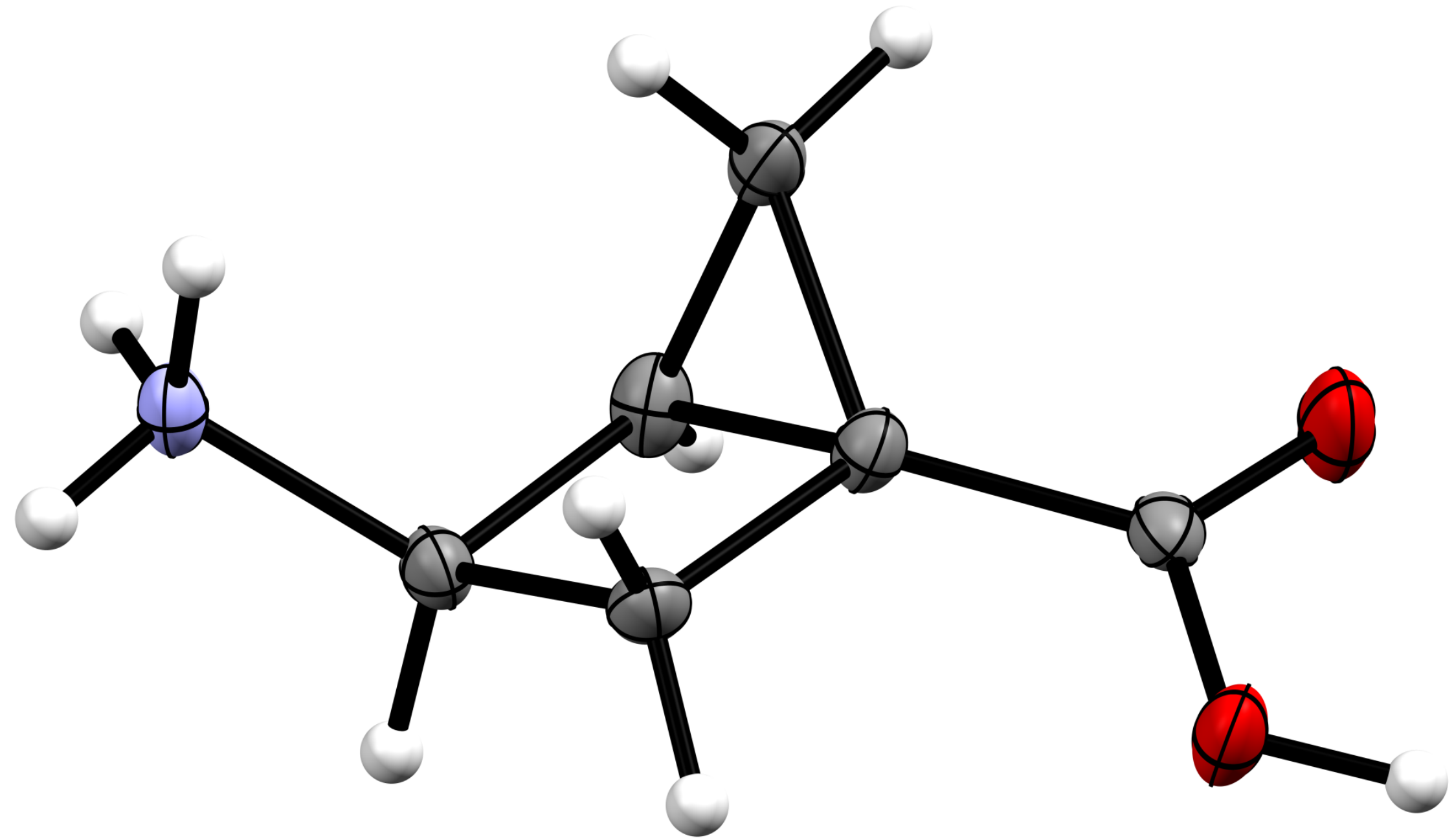

$(1 S, 2 R, 4 R)$-3-aminobicyclo[2.1.0]pentane-1-carboxylic acid hydrochloride (1S,2R,4R)-21 ORTEP diagrams

(Thermal ellipsoids are shown at $30 \%$ probability level) 


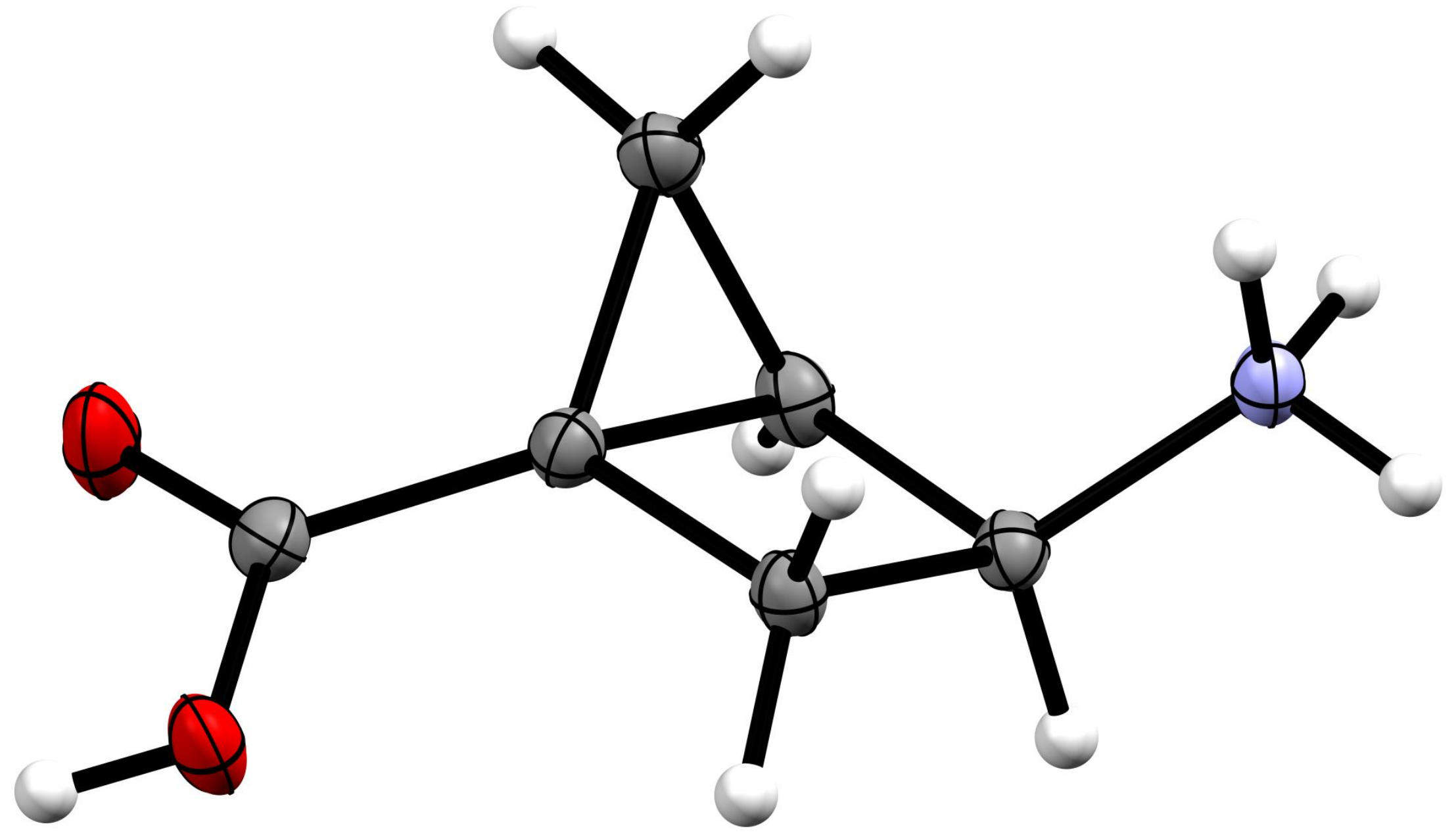

$(1 R, 2 S, 4 S)$-3-aminobicyclo[2.1.0]pentane-1-carboxylic acid hydrochloride (1R,2S,4S)-21 ORTEP diagrams

(Thermal ellipsoids are shown at $30 \%$ probability level) 


\section{$\mathrm{X}$-Ray diffraction studies of 10b, 17b, $(1 S, 2 S, 4 R)-21,(1 R, 2 R, 4 S)-21,(1 S, 2 R, 4 R)-22,(1 R, 2 S, 4 S)-22$.}

X-Ray diffraction studies were performed with single crystals of housane derivatives obtained by slow evaporation of their solutions: cis phenyl-substituted housane carboxylic acid 10b (in $t$-BuOMe - hexanes), its trans isomer 17b (in THF), as well as amino acid hydrochlorides $(1 S, 2 S, 4 R)-\mathbf{2 1} \cdot \mathrm{HCl} \cdot \mathrm{H}_{2} \mathrm{O}$ (in $\mathrm{MeCN}-$ $\mathrm{EtOH}),(1 R, 2 R, 4 S)-\mathbf{2 1} \cdot \mathrm{HCl} \cdot \mathrm{H}_{2} \mathrm{O}$ (in $\left.\mathrm{MeCN}-\mathrm{EtOH}\right) ;(1 S, 2 R, 4 R)-\mathbf{2 2} \cdot \mathrm{HCl}$ (in $\left.\mathrm{MeOH}\right)$, and $(1 R, 2 S, 4 S)-\mathbf{2 2} \cdot \mathrm{HCl}($ in $\mathrm{MeCN}-\mathrm{EtOH})$.

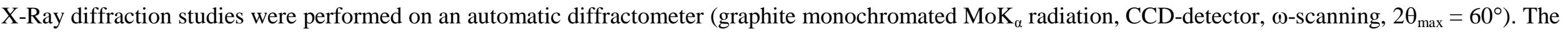

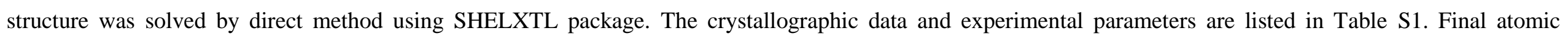

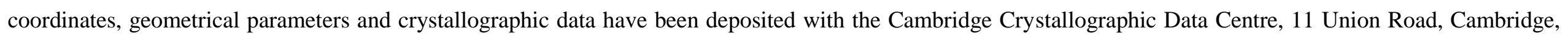
CB2 1EZ, UK (fax: +44 1223 336033; e-mail: deposit@ccdc.cam.ac.uk). The deposition numbers are given in Table S1.

Table S1. The crystallographic data and experimental parameters for compounds

$\mathbf{1 0 b}, \mathbf{1 7 b},(1 S, 2 S, 4 R)-\mathbf{2 1},(1 R, 2 R, 4 S)-\mathbf{2 1},(1 S, 2 R, 4 R)-\mathbf{2 2},(1 R, 2 S, 4 S)-\mathbf{2 2}$

\begin{tabular}{|c|c|c|c|c|c|c|}
\hline Parameter & $10 b$ & $17 \mathbf{b}$ & $(1 S, 2 S, 4 R)-21$ & $(1 R, 2 R, 4 S)-21$ & $(1 S, 2 R, 4 R)-22$ & $(1 R, 2 S, 4 S)-22$ \\
\hline \multicolumn{7}{|c|}{ Unit cell dimensions } \\
\hline $\mathrm{a}, \AA$ & $10.7797(7)$ & $6.0802(8)$ & $6.3744(2)$ & $6.3758(4)$ & $6.1443(5)$ & $6.1463(3)$ \\
\hline $\mathrm{b}, \AA$ & $21.3371(13)$ & $7.0558(8)$ & $6.7541(2)$ & $6.7491(5)$ & $7.4411(7)$ & $7.4304(3)$ \\
\hline c,,$\AA$ & $8.7302(5)$ & $22.379(3)$ & $20.5822(6)$ & $20.5738(12)$ & $8.5384(7)$ & $8.5433(4)$ \\
\hline$\alpha, \circ$ & 90 & 90 & 90 & 90 & 90 & 90 \\
\hline$\beta, \circ$ & $102.503(3)$ & $93.955(4)$ & 90 & 90 & $93.856(4)$ & $93.884(3)$ \\
\hline$\gamma,{ }^{\circ}$ & 90 & 90 & 90 & 90 & 90 & 90 \\
\hline $\mathrm{V}, \AA^{3}$ & $1960.4(2)$ & $957.8(2)$ & $886.13(5)$ & $885.31(10)$ & $389.49(6)$ & $389.27(3)$ \\
\hline
\end{tabular}




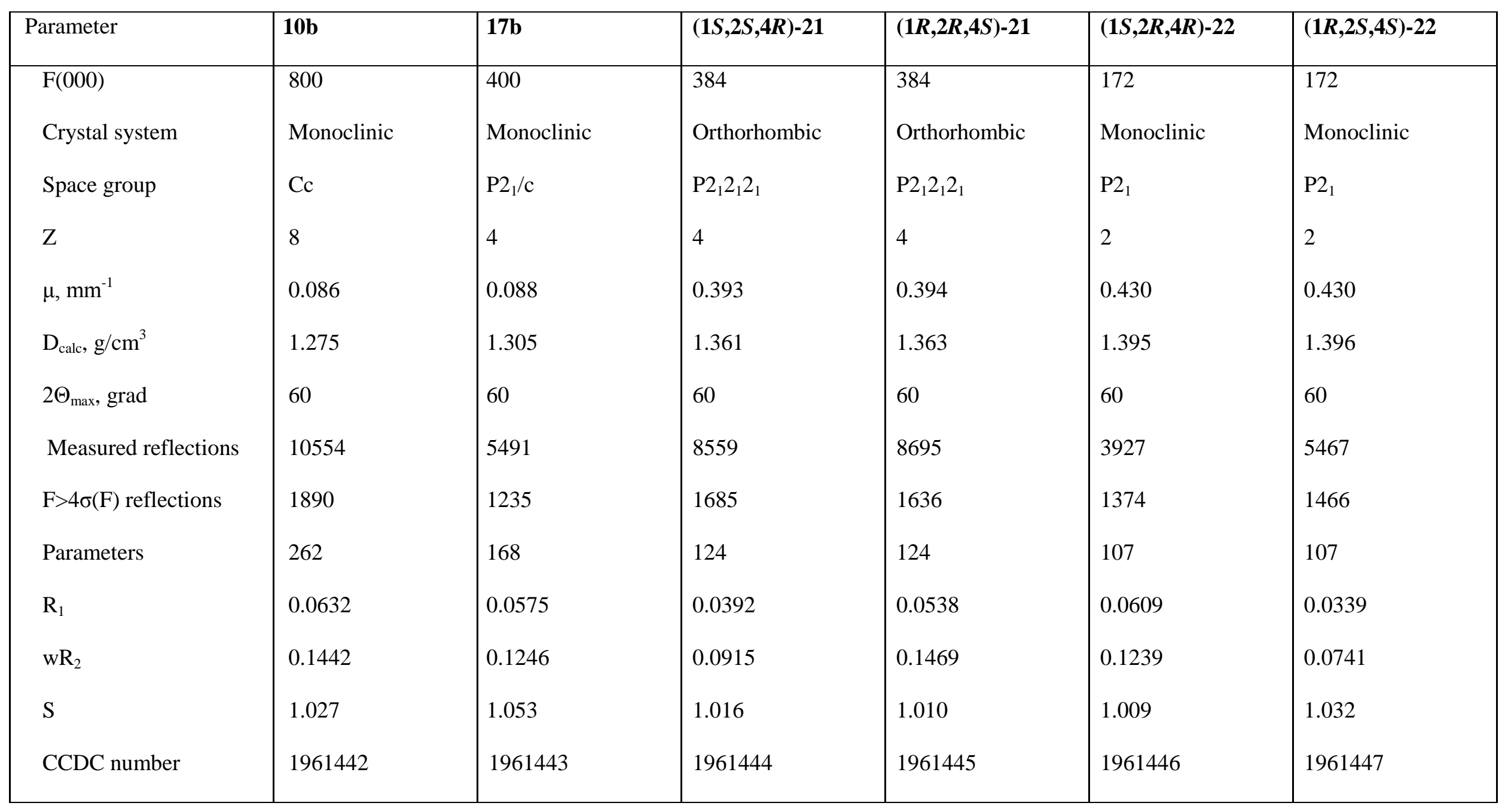

